NASA Contractor Report 170416

NASA CR-170,416

NASA-CR-170416

19840013483

\title{
An Optimal Control Approach to Pilot/Vehicle Analysis and the Neal-Smith Criteria
}

Barton J. Bacon and David K. Schmidt

Grant NAG4-1

April 1984

LBBAARY ROPY

s.6 9946

LANGLIEY RESLARCH CENTER

LIBRARY, NASA

IMAMTON, VIRGINIA 


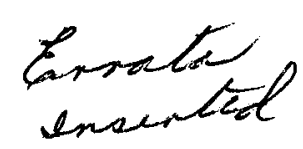

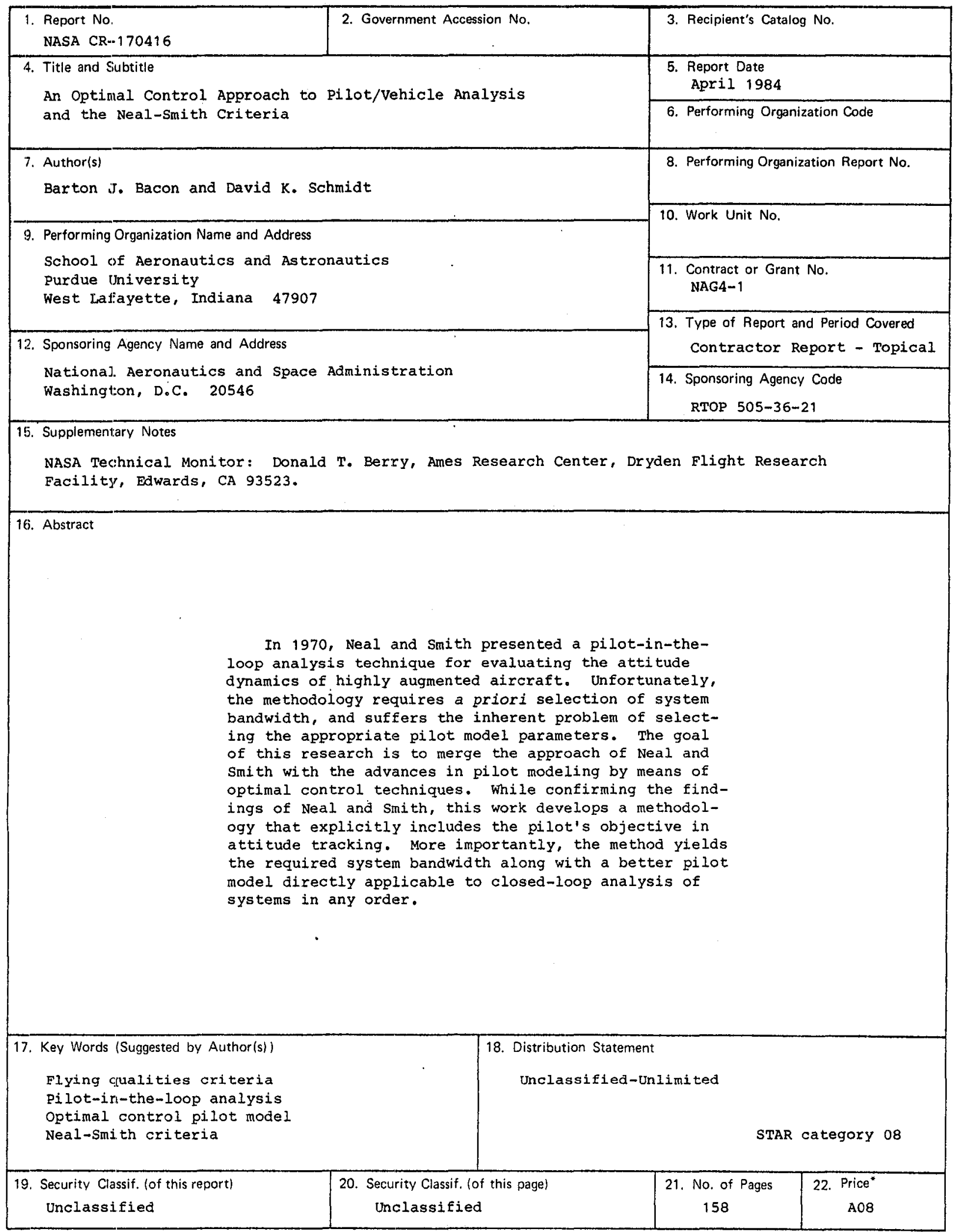

*For sale by the National Technical Information Service, Springfield, Virginia 22161. 
NASA Contractor Report 170416

\section{An Optimal Control Approach to Pilot/Vehicle Analysis and the Neal-Smith Criteria}

Barton J. Bacon and David K. Schmidt

School of Aeronautics and Astronautics, Purdue University, West Lafayette, Indiana

Prepared for

Ames Research Center

Dryden Flight Research Facility

Edwards, California

under Grant NAG4-1

1984

\section{N/SA}

National Aeronautics and

Space Administration

Ames Research Center

Dryden Flight Research Facility

Edwards, California 93523 
This Page Intentionally Left Blank 
TABLE OF CONTENTS

Page

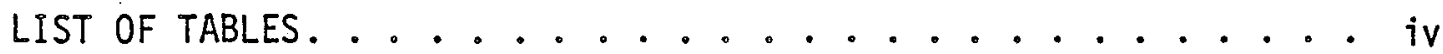

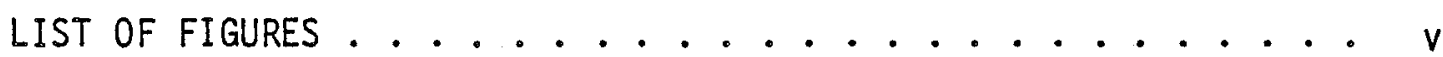

LIST OF SYMBOLS .................... . ix

ABSTRACT................................

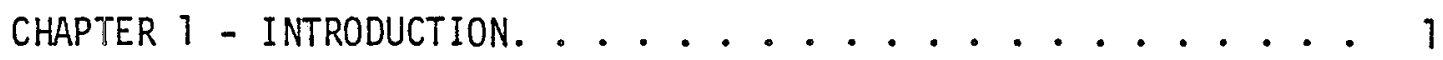

CHAPTER 2 - THE ANALYSIS OF NEAL AND SMITH. . ...... 4

CHAPTER 3 - THE OPTIMAL CONTROL MODEL . . . . . . . 22

3.1 Optimal Control of a Linear System with

Time Delay and Measurement Noise. ......... 24

3.2 The Optimal Control Pilot Model . . . . . . . . 36

3.3 Pilot's Frequency Response. . . . . . . . . . . 42

CHAPTER 4 - METHODOLOGY AND RESULTS . . . . . . . 46

4.1 Basic Hypothesis. . . . . . . . . . . 47 47

4.2 Modeling the Task ............. 49

4.3 Analys is Technique. . . . . . . . . . . 55 53

4.4 Results from the Methodology. .......... 63

CHAPTER 5 - SUMMARY

5.1 Summary and Conclusion. ........... 73

5.2 Areas of Further Study. . . . . . . . . . . 74

LIST OF REFERENCES. . . . . . . . . . . . 76

APPENDIX A - SIMPLIFYING THE TOTAL COST OF SECTION 3.2. . . . 77

APPENDIX B - FORMULATION OF THE STATE COVARIANCE MATRIX

FOR SECTION $3.2 \ldots \ldots \ldots . \ldots . \ldots . \ldots$

APPENDIX C - SUMMARY OF OCM RESULTS .......... 83 


\section{LIST OF TABLES}

Table

2.1 Configuration Summary. ................ 5

4.1 Baseline Pilot Model .............. 52

4.2 RMS Comparison for Configuration 2A. . . . . . . 57

4.3 Selected Frequencies ............... 58

4.4 Summary of Results Obtained for the 8 Basic

Configurations ............... 68

4.5 Summary of Results Obtained for Configurations

of Group 1.................. 69

4.6 Summary of Results Obtained for Configurations

of Group 2.............................. 70 


\section{LIST OF FIGURES}

Figure

Page

2.1 Block Diagram of Standard Configurations. ....... 5

2.2 Cooper-Harper Rating Scale. . . . . . . . . 6

2.3 PIO Tendency Rating Scale ........... 6

2.4 Classical Model Structure . . . . . . . . . 9

2.5 Nea 1-Smith Pilot Strategy in Tracking......... 11

2.6 Nichols Chart Showing Performance Standards and a

Sample Amplitude Phase Curve. . . . . . . . . . 13

2.7 Overlay of Amplitude Phase Curves for "Optimum".

Pilot Compensation. ............ 15

2.8 Open-Loop Bode Characteristic for Configuration 2G. . . . 16

2.9 Uncompensated/Compensated Amplitude-Phase Curve for Configuration $2 G$. . ............. 18

2.10 Gain-Adjusted, Compensated Amplitude-Phase Curve for

Configuration 2G. . . . . . . . . . . 19

2.11 Neal-Smith Results. . . . . . . . . 20

3.1 Optimal Control Pilot Mode1 ........... 23

3.2a Estimator Orthogonally Projects the Delayed State on the Subspace of Observations. ......... 27

3.2b Predictor Orthogonally Projects the Current Estimate on the Subspace of Delayed Estimates. . . . . . . . 27

4.1 Model Schematic Comparison. ............ 55

4.2 Pilot Frequency Response. ............ 61

4.3 System Frequency Response . . . . . . . . . . . 62

4.4 Pilot Rating/Bandwidth Correlation. . . . . . . . 65 
Figure

4.5 Results of Optimal Control Anajysis. . . . . . . . 66

C. 1 Configuration TA/Pilot Frequency Response. . . . . . 83

C.2 Configuration TA/System Frequency Response . . . . . . 84

C.3 Configuration IA/Corrected System Frequency Response . . 85

C.4 Configuration 1B/Pilot Frequency Response. . . . . . . 86

C.5 Configuration 1B/System Frequency Response . . . . . . 87

C.6 Configuration TB/Corrected System Frequency Response . . . 88

C. 7 Configuration TC/Pilot Frequency Response. . . . . . 89

C. 8 Configuration 1C/System Frequency Response . . . . . . 90

C.9 Configuration IC/Corrected System Frequency Response . . . 91

C. 10 Configuration 10/Pilot Frequency Response. . . . . . . 92

C. 11 Configuration 1D/System Frequency Response . . . . . . . 93

C. 12 Configuration 10/Corrected System Frequency Response . . . 94

C. 13 Configuration IE/Pilot Frequency Response. . . . . . . . 95

C. 14 Configuration 1E/System Frequency Response . . . . . . . 96

C. 15 Configuration TE/Corrected System Frequency Response . . . 97

C. 16 Configuration 1F/Pilot Frequency Response. . . . . . . 98

C. 17 Configuration 1F/System Frequency Response . . . . . . 99

C.18 Configuration 1G/Pilot Frequency Response. . . . . . 100

C. 19 Configuration 1G/System Frequency Response ........ 101

C. 20 Configuration 2A/Pilot Frequency Response. . . . . . . 102

C. 21 Configuration 2A/System Frequency Response . . . . . . 103

C. 22 Configuration 2A/Corrected System Frequency Response . . . 104

C. 23 Configuration 2B/Pilot Frequency Response. . . . . . . 105

C. 24 Configuration 2B/System Frequency Response . . . . . . 106 
Figure

Page

C.25 Configuration 2B/Corrected System Frequency Response. . 107

C. 26 Configuration 2C/Pilot Frequency Response . . . . . 108

C.27 Configuration 2C/System Frequency Response. . . . . 109

C.28 Configuration 2C/Corrected System Frequency Response. - . 110

C.29 Configuration 2D/Pilot Frequency Response . . . . . . 111

C.30 Configuration 20/System Frequency Response. . . . . . 112

C.31 Configuration 20/Corrected System Frequency Response. . - 113

C.32 Configuration 2E/Pilot Frequency Response . . . . . . 114

C.33 Configuration 2E/System Frequency Response. . . . . . 715

C.34 Configuration 2E/Corrected System Frequency Response. . . 116

C.35 Configuration 2F/Pi lot Frequency Response . . . . . . 117

C.36 Configuration 2F/System Frequency Response. . . . . . 118

C.37 Configuration 2F/Corrected System Frequency Response. . - 119

C.38 Configuration 2G/Pilot Frequency Response . . . . . . 120

C.39 Configuration 2G/System Frequency Response. . . . . . 121

C. 40 Configuration 2G/Corrected System Frequency Response. . 122

C.41 Configuration 2H/Pilot Frequency Response ... . . . . 123

C.42 Configuration 2H/System Frequency Response. . . . . . . . 124

C.43 Configuration $2 \mathrm{H} /$ Corrected System Frequency Response. - 125

C.44 Configuration 2I/Pilot Frequency Response . . . . . . 126

C.45 Configuration 2I/System Frequency Response. . . . . . . 127

C. 46 Configuration 2I/Corrected System Frequency Response. . - 128

C.47 Configuration 2J/Pilot Frequency Response . . . . . . 129

C.48 Configuration 2J/System Frequency Response. . . . . . 130

C.49 Configuration 3A/Pilot Frequency Response . . . . . . 131 
Figure

C. 50 Configuration 3A/System Frequency Response. . . . . . 132

C.51 Configuration 3A/Corrected System Frequency Response. - . 133

C.52 Configuration 4A/Pilot Frequency Response . . . . . . 134

C.53 Configuration 4A/System Frequency Response. . . . 135

C.54 Configuration 4A/Corrected System Frequency Response. - 136

C.55 Configuration 5A/Pilot Frequency Reseponse. . . . . 137

C.56 Configuration 5A/System Frequency Response. . . . . . 138

C.57 Configuration 5A/Corrected System Frequency Response. - . 139

C.58 Configuration 6C/Pilot Frequency Response ...... 140

C.59 Configuration 6C/System Frequency Response. . . . . . 141

C.60 Configuration 6C/Corrected System Frequency Response. - 142

C.61 Configuration 7C/Pilot Frequency Response ....... 143

C.62 Configuration 7C/System Frequency Response. . . . . . . 144

C. 63 Configuration 7C/Corrected System Frequency Response. - 145

C.64 Configuration 8A/Pi]ot Frequency Response ...... 146

C.65 Configuration 8A/System Frequency Response. . . . . . 147

C. 66 Configuration 8A/Corrected System Frequency Response. - 148 


\section{LIST OF SYMBOLS}

Symbol

$A, A_{1}, A_{0}, A_{c}, A_{\text {veh }}$
$b, b_{0}, b_{1}, b_{\text {veh }}$
$B$
$C, C_{0}, c_{1}$
$\bar{e}(t)$
$\bar{e}_{p}(t)$
$E\{\cdot\}$
$f$
$F_{s}$

g

$\bar{H}_{p}(s)$

$H_{\varepsilon}, H_{\dot{\varepsilon}}, H_{\theta}, H_{\dot{\theta}}$

$\mathrm{H}_{\mathrm{a}}(\mathrm{s})$

I

$\mathrm{J}, \mathrm{J}_{1}, \mathrm{~J}_{2}$

$J_{p}$

$k, k_{0}$

$L^{*}, \ell, e^{*}$,

$N\left(\sigma_{i}, \alpha_{j}\right)$

Q
Meaning

Plant matrices

Control vectors

Control matrices

Output matrices

Estimation error

Prediction error

Expected value operator

Fraction of pilot attention

Control input (stick force)

Pilot weighting on input rate

Pilot transfer matrix

Elements of $\bar{H}_{p}(s)$

Vehicle's transfer function

Identity matrix

Objective functions producing the same control $\bar{u}$

Pilot objective function

Riccati gain matrices

Optimal control gain matrices

observation threshold describing function amplitude ratio

Weighting matrix on states 
Symbol

$\mathrm{Q}_{y}$

$\bar{r}(t)$

r

R

$S(\cdot)(\omega)$

$t$

$\bar{u}$

$u_{c}$

$u_{p}$

$v_{m}$

$v_{m}$

$\bar{v}$

V

$\bar{w}, \bar{w}_{0}, w_{y}$

W, $W_{1}$

$\tilde{\bar{w}}, \overline{\bar{w}}_{2}$

$\tilde{W}_{3} \tilde{W}_{2}$

$\bar{x}, \bar{x}_{c}, \bar{x}$

$\hat{\bar{x}}(t)$

$\bar{y}, \bar{y}_{p}$

$\bar{y}_{u}(t)$

$\alpha_{i}$

$\dot{\gamma}$

$\varepsilon, \theta_{e}$
Meaning

Pilot weighting matrix on outputs

Stochastic part of $\hat{x}(t-\tau)$

Pilot weighting on input

Weighting matrix on control $u$

Spectral density of $(\cdot)$

Time

Control input vector

Pilot's commanded control input

Pilot's. final control input

Pilot's neuromotor noise

Covariance matrix of pilot's neuromotor noise

Observation noise vector

Covariance of observation noise

Plant process driving noises

Covariance matrices of plant driving noise

Plant noises driving, respectively, the estimated and predicted state

Covariance matrix associated with $\tilde{\bar{w}}$ and $\tilde{\bar{w}}_{2}$

State vectors

Least mean square estimate of $\bar{x}(t)$

Observation vector

Deterministic part of $\hat{\bar{x}}(t-\tau)$

Threshold level for the ith observed variable

Least-mean-square prediction of $\hat{\bar{x}}(t), \hat{\bar{x}}(t)$

Tracking error 
Symbol

$\theta$

$\theta_{\mathrm{C}}$

$\bar{\xi}$

$\rho_{i}$

$\sigma(\cdot)$

$\bar{\Sigma}, \bar{\Sigma}_{1}$

$\tau$

${ }^{\mathrm{T}} N$
Meaning

Pitch attitude

Commanded pitch attitude

State vector for predictor dynamics

Basic noise-to-signal ratio for ith observed variable

Root-mean-square of $(\cdot)$

Solution matrix of Kalman filter variance equation (steady state)

Time delay

Neuromotor time constant 
This Page Intentionally Left Blank 


\section{CHAPTER 1}

\section{INTRODUCTION}

In the past, the longitudinal handling qualities of an aircraft were determined almost entirely by the modal characteristics of the classical rigid body modes (short period and phugoid). These modes dominate the conventional aircraft's dynamics, and their modal parameters (i.e. damping and natural frequency) exhibit a definite correlation with pilot opinion ratings. Unfortunately, beyond the rea $7 \mathrm{~m}$ of conventional aircraft, criteria based on these parameters alone are inadequate. The addition of other modes, whether they be due to structural dynamics or to augmentation has been shown to seriously affect pilot opinion rating.

In the early $70^{\prime} \mathrm{s}, \mathrm{Neal}$ and $\mathrm{Smith}{ }^{[1]}$ hypothesized that "pilot rating is a strong function of the pilot's compensation required to achieve good low frequency performance and the pilot/vehicle oscillatory tendencies that resulted." Equating good tracking performance with closed-loop frequency response characteristics, they devised a "pilot-in-the-1oop" analysis capable of explaining problems the pilot might: observe in pitch attitude tracking. Unfortunately, the method has a few drawbacks.

These drawbacks are typical of classical pilot models applied to handling qualities prediction in that even if given a hypothesized form of the pilot's control-loop structure, the task still includes 
selecting parameters such that the resulting model mimics the inputoutput behavior of the pilot. And herein lies the difficulty with pilot transfer functions that are often impossible to measure, and methods of pilot-model parameter selection that often appear to be dependent on "miracles and black magic."

Once the barrier of "conjuring-up" parameters is broken, these methods are usually straightforward, with elements of the closed-loop performance and the pilot model utilized to predict/explain pilot opinion ratings. However, breaking this barrier still remains the key to successfully gauging pilot/vehicle performance.

To side step the "black magic" of the classical approach, consider another development of the early $70^{\prime} \mathrm{s}$; that is the optimalcontrol model (OCM) of human behavior. The $\mathrm{OCM}_{3}$ a product of optimal control and estimation theory, needs no a priori knowledge of the pilot loop structure. Also, the parameters of the multi loop pilot transfer functions can be computed as part of the optimal control solution. Of course, these benefits are partially offset since pilot strategy, as reflected by cost functional weightings of the quantities to be minimized, must be determined.

The objective of this study, then, is a better pilot modelling technique via optimal control theory, and still conceptually approach the pilot-rating-prediction problem in a manner similar to Neal and Smith.

This thesis is divided into the following chapters to provide the background material and the methodology needed for the new approach in handling qualities prediction. Chapter 2 presents the Neal and 
3

Smith method by considering their interpretation of pilot strategy and the factors influencing pilot opinion rating. Chapter 3 gives a detailed account of the optimal control model's development and strucktore. And, Chapter 4 focusing on the possibility of implementing the optimal-control model in the analysis, synthesizes the tracking task in the context of the OCM and presents the proposed OCM analysis, complete with a discussion of the results. Chapter 5 finally presents the summary and conclusions of the research. 
CHAPTER 2

THE ANALYSIS OF NEAL AND SMITH

The Neal and Smith's investigation of the early 70's had a twofold objective: to provide data on the effects of Flight Control system (FCS) dynamics and to develop a design criterion capable of pinpointing pilot problem areas encountered in performing a given task.

To meet the first objective, two pilots evaluated a total of 51 basic configurations of $\mathrm{FSC} /$ short period dynamics in flight. A block diagram of the vehicle dynamics and a listing of parameters defining 23 of the configurations simulated in flight are presented, respectively, in Figure 2.1 and Table 2.1. These in-flight simulations provided pilot comments concerning the effects of adding a single FCS zero and a single FCS pole to eight baseline short period configurations engaged in combat-related manuevers. In addition to comments, the pilots assigned an overall pilot rating (Cooper-Harper) and a PIO (pilotinduced-oscillation) rating to each configuration.

The Cooper-Harper Pilot Rating Scale, shown in Figure 2.2 represents a numerical summary of an aircraft's suitability to perform a given task. The ten-point scale rates an aircraft based on the level of system controllability, the attainable level of performance, and the required pilot compensation. Incidentally, pilot rating is also related to the PIO rating that quantifies the aircraft's tendency to oscillate during the performance of the task. Truly, an aircraft's 
Stick

Force

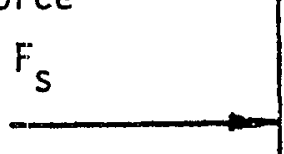

$$
\frac{k_{\theta}\left(\tau_{1} s+1\right)\left(\tau_{\theta_{2}} s+1\right)}{s\left(\tau_{2} s+1\right)\left(\frac{s^{2}}{\omega_{3}^{2}}+\frac{2 \tau_{3}}{\omega_{3}} s+1\right)\left(\frac{s^{2}}{\omega_{s p}^{2}}+\frac{2 i_{s D}}{w_{s p}} s+1\right)}
$$

Pitch

Attitude

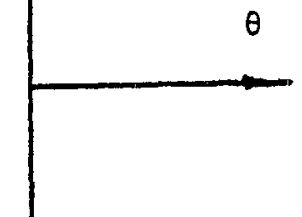

Figure 2.1 Block Diagram of Standard Configuration

Table 2.1 Configuration Sumary

\begin{tabular}{|c|c|c|c|c|c|}
\hline Cont. & $1 / \tau_{1}$ & $1 / \tau_{\theta_{2}}$ & $1 / \tau_{2}$ & $\omega_{s p} / \tau_{s p}$ & $i_{3} / \tau_{3}$ \\
\hline $1 A$ & 0.5 & 1.25 & 2 & $2.2 / .69$ & \\
\hline is & 2.0 & & 5.0 & & \\
\hline IC & 2.0 & & 5.0 & & 18.1 .75 \\
\hline 10 & - & & $\infty$ & & \\
\hline IE & & & 5.0 & & \\
\hline IF & & & 2.0 & & \\
\hline $1 G$ & $\downarrow$ & & 0.5 & $t$ & \\
\hline $2 A$ & 2.0 & & 5.0 & $4.9 / .70$ & \\
\hline $2 B$ & 2.0 & & 5.0 & 1 & 16.1 .75 \\
\hline $2 C$ & 5.0 & & 12.0 & & . \\
\hline 20 & $\because$ & & - & & \\
\hline $2 E$ & & & 12.0 & & \\
\hline $2 F$ & & & 5.0 & & \\
\hline 26 & & & 5.0 & & 16.1 .75 \\
\hline $2 \mathrm{H}$ & & & 2.0 & & \\
\hline $2 !$ & & & 2.0 & & 16.1 .75 \\
\hline $2 \mathrm{~s}$ & & & 0.5 & 1 & \\
\hline $3 A$ & & & $i$ & $9.7 / .63$ & \\
\hline $4 A$ & & & & $5.0 / .28$ & \\
\hline $5 A$ & & 4 & & $5.1 / .13$ & \\
\hline $6 C$ & & 2.2 & & $3.4 / .67$ & \\
\hline $7 C$ & & & & $7.3 / .73$ & \\
\hline $8 A$ & \} & 8 & $Y$ & $16.5 ! .69$ & \\
\hline
\end{tabular}




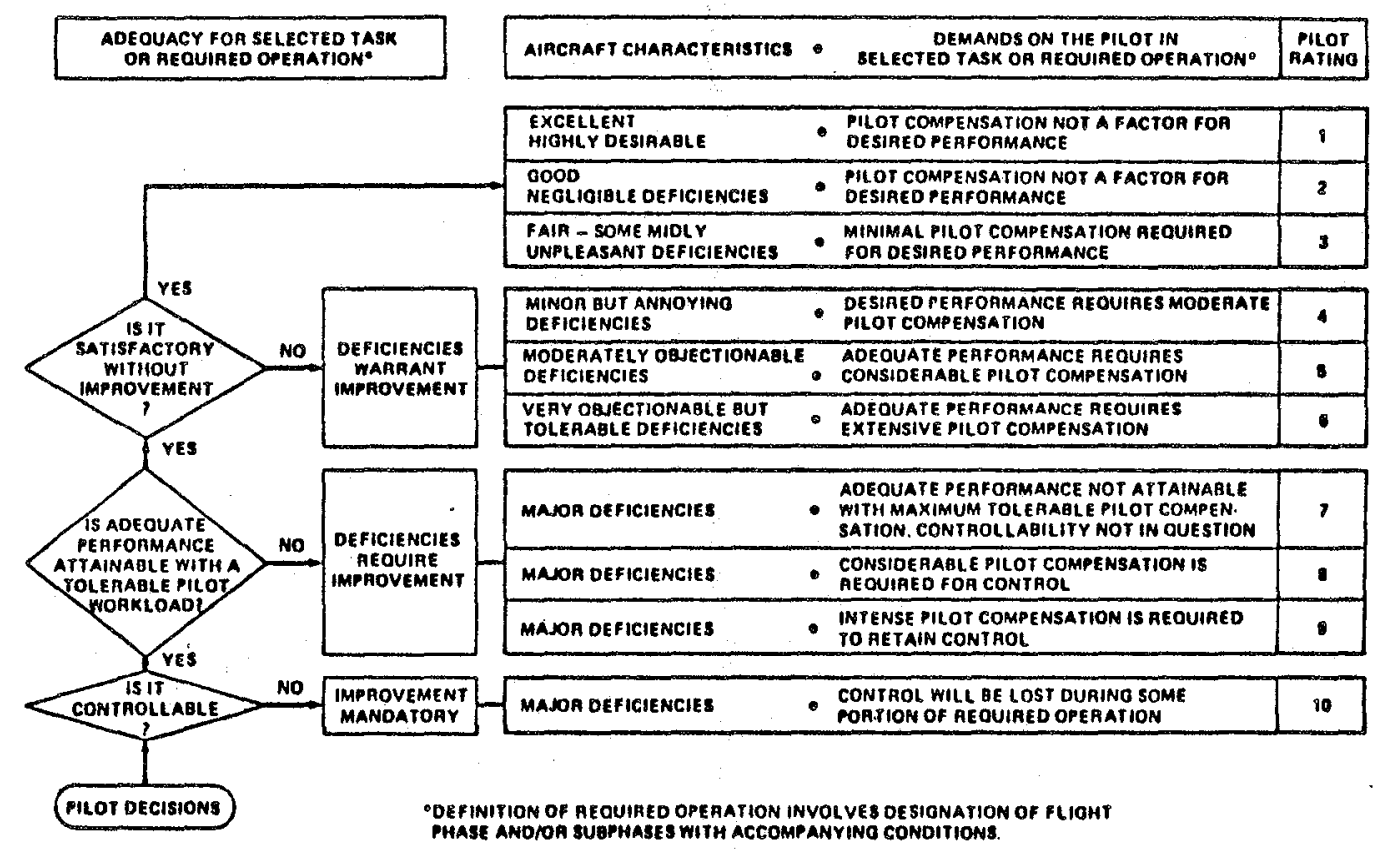

Figure 2.2 Cooper-Harper Rating Scale

\begin{tabular}{|c|c|}
\hline DESCAIMTION & $\begin{array}{l}\text { NUMERICAL } \\
\text { MATINC }\end{array}$ \\
\hline $\begin{array}{l}\text { NO TENOENCY FOR MLOT TO INOUCE LNCESIAABLI } \\
\text { MOTIONS }\end{array}$ & 9 \\
\hline 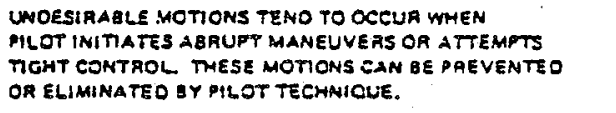 & 2 \\
\hline 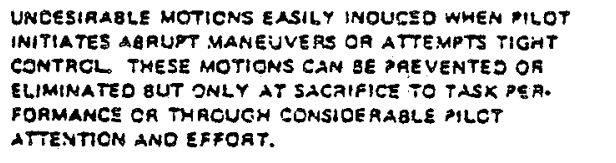 & 3 \\
\hline 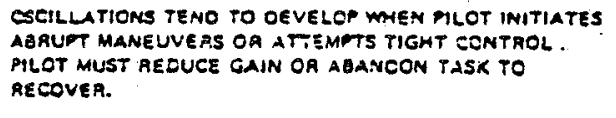 & 4 \\
\hline 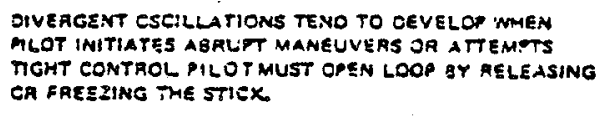 & 5 \\
\hline 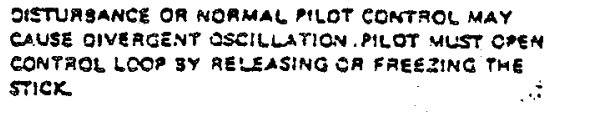 & 8 \\
\hline
\end{tabular}

Figure 2.3 PIO Tendency Rating Scale 
oscillatory tendencies can seriously affect the pilot's attainable level of performance. Figure 2.3 presents the six-point PIO Tendency Rating Scale. The descriptions associated with the numerical ratings will play an important role in evaluating the results of the proposed alternate method to be presented later.

For now, these descriptions and their rating scales simply enumerate problem areas the pilot encounters. Thus, predicting these ratings would achieve Neal and Smith's second goal. And since pilot rating is intimately correlated with PIO rating, predicting the former would be sufficient to achieve the objective.

Returning to the first objective, preliminary results, comprised of pilot comments, pilot ratings, and PIO ratings for a cross section of the aircraft flight tested, concluded that the addition of FCS dynamics "can drastically alter the airplane's short-period response." A group of aircraft, containing the same short-period characteristics, but a varying set of FCS pole-zero pairs, demonstrated the degrading effect certain FCS dynamics can have on pilot opinion. As an example consider the aircraft of Group 2. Here, the basic short period configuration $2 D$ received a good pilot rating of 2.5 . However, upon the inclusion of configuration 2G's FCS dynamics, the pilot rating fell to 8 , a poor rating. A similar trend exists for Group l's dynamics, but in one case, IB, the pilot rating improved with added FCS dynamics. Evidently, short period characteristics alone cannot adequately predict an aircraft's handling qualities.

Moreover, the difficulties of using existing open-loop criterion to explain all the results of this experiment led to the development 
of an alternate approach: - the "pilot-in-the-loop" analysis. Based on pilot comments, this approach assumes that the pilot opinion rating is largely determined by the precision of pitch attitude control. In particular, pitch attitude tracking, the ability to rapidly acquire and track distant air and ground targets, became the backdrop of the Neal and Smith Analysis.

To analyze the effects of various FCS dynamics on performing the proposed task, a suitable model of pitch attitude tracking was sought. Neal and Smith selected the compensatory tracking model of Figure 2.4. The pilot, modelled as a simple lead-lag filter with a time delay and gain, is considered to operate only on the difference between the aircraft's attitude and the commanded attitude. The pilot's time delay (taken as $0.3 \mathrm{sec}$ ) included the effects of perceptual delays and neuromuscular lags associated with most manual control systems.

Given this pilot's controller structure, the need turns to finding the pilot parameters $\left(K_{p}, T_{p_{1}}, T_{p_{2}}\right)$, and Neal and Smith went on to propose the closed loop characteristics representative of a pilot's perception of tracking strategy. To aid in the coming discussion the following terminology should be noted

(1) $\frac{\theta}{F_{S}}$ is the open-10op transfer function of the aircraft plus FCS

(2) $\frac{\theta}{\theta_{e}}$ is the open-loop transfer function of the aircraft plus FCS plus pilot

(3) $\frac{\theta}{\theta_{c}}$ is the closed-loop transfer function of the aircraft plus FSC plus pilot, which is related to $\frac{\theta}{\theta_{e}}$ by 


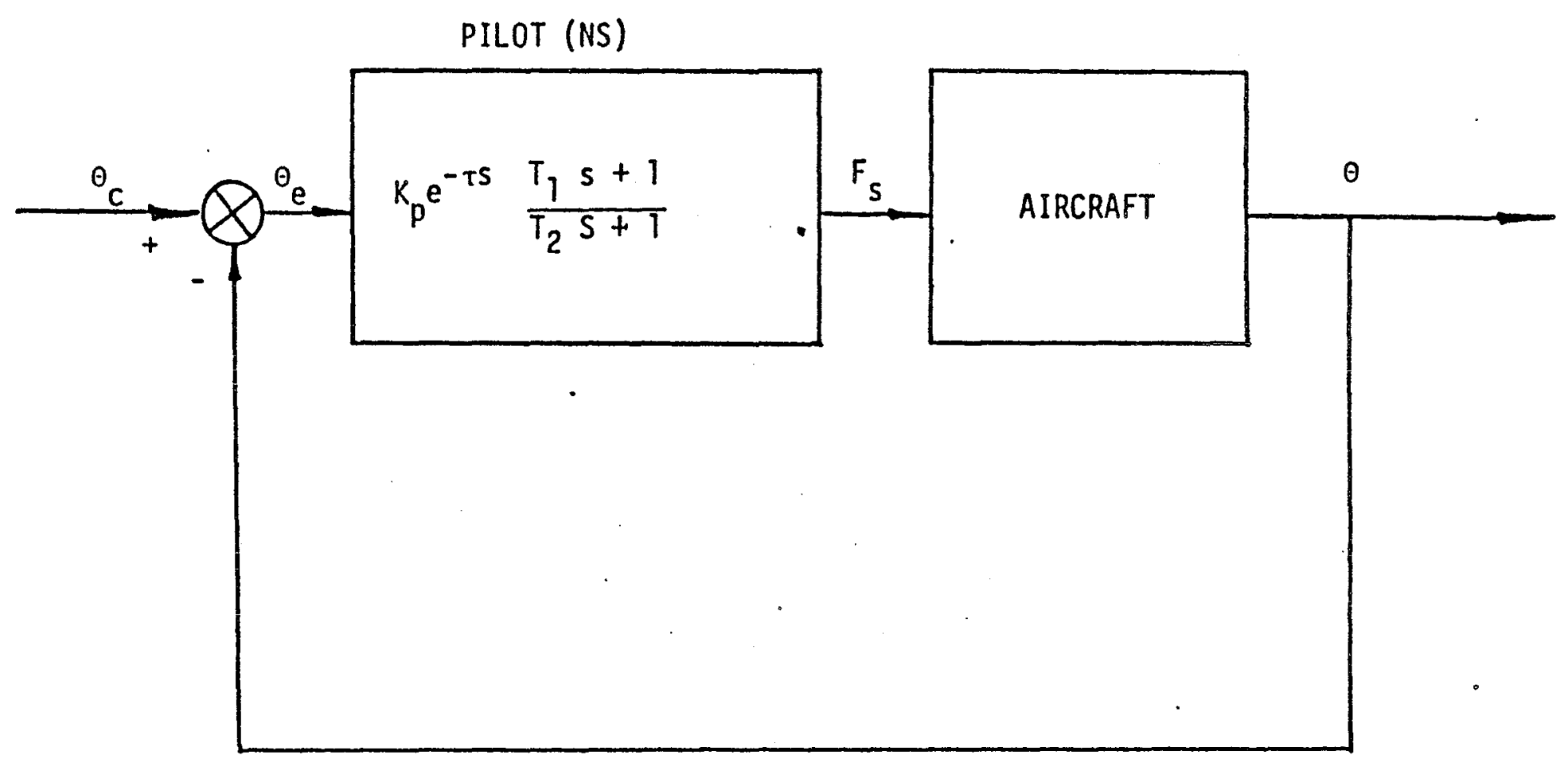

Figure 2.4 Classical Model Structure 


$$
\frac{\theta}{\theta_{c}}=\frac{\frac{\theta}{\theta_{e}}}{1+\frac{\theta}{\theta_{e}}}
$$

With the ultimate goal of understanding how the pilot actually flies the tracking mission, the investigation began by asking the fundamental question; What is the pilot actually trying to do?

Clearly, the pilot wants to acquire the target quickly and predictably, with a minimum of overshoot and oscillation. Referring to Figure 2.5, the analysis interpreted the phrase "To acquire the target quickly and predictably" as meaning the pilot wants to attain a certain bandwidth and below this frequency, keep the magnitude $\left(\frac{\theta}{\theta_{c}}\right)$ relatively close to $0 \mathrm{~dB}$. Bandwidth (BW) was defined as the frequency at which the closed-loop phase angle of $\left(\frac{\theta}{\theta_{c}}\right)$ is -90 degrees. Neal and Smith continued the interpretation of this phrase by correlating the desire "to minimize oscillation" with minimizing the closed-loop resonant peak $\left|\frac{\theta}{\theta_{c}}\right|_{\max }$. They noted typically that pilot strategy was a tradeoff between striving for acceptable low frequency performance and eliminating the accompanying oscillations.

The Neal-Smith investigation concluded that "pilot rating is a function of the compensation required to achieve good low frequency performance and the oscillatory tendencies that result." The analysis defined the pilot compensation in terms of the phase angle

$$
\xi_{p c}=\left.\Varangle\left(\frac{T_{p 1} s+1}{T_{p 2} s+T}\right)\right|_{\omega=B W}
$$

in their pilot model. It is frequently interpreted as a measure of the pilot's physical and mental "workload" required. 


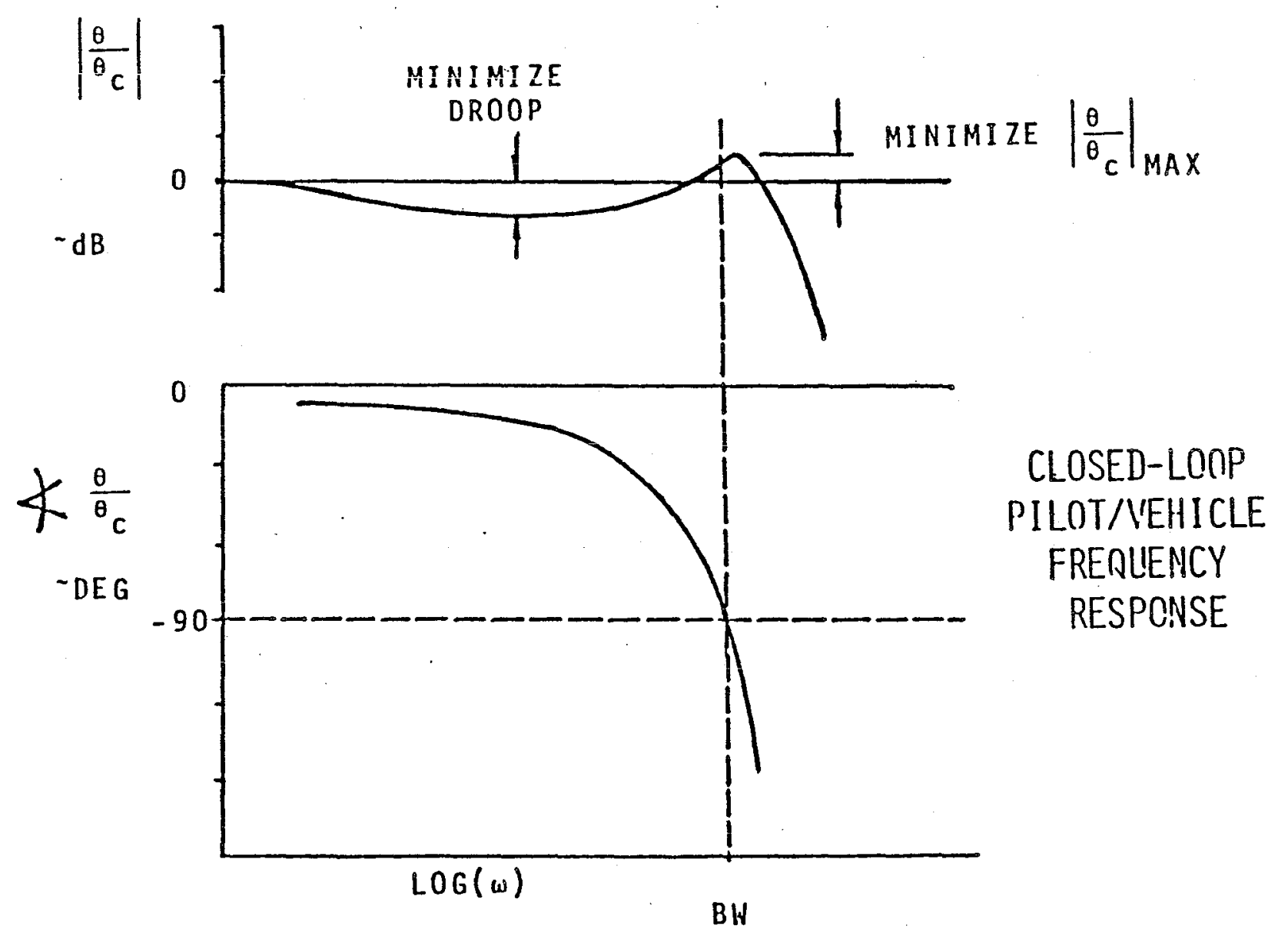

Figure 2.5 Nea1-Smith Pilot Strategy in Tracking 
Therefore, the key to the analysis centered on determining the two measures, pilot compensation and magnitude of the resonant peak, hypothesized to correlate with pilot rating. To do so, pilot parameters $\left(K_{p}, T_{p 1}, T_{p 2}\right)$ were chosen to reflect the pilot's strategy by meeting the following empirical closed-10op performance standards:

(1) A bandwidth of $3.5 \mathrm{rad} / \mathrm{sec}\left(\Varangle\left(\theta / \theta_{c}\right) \geq-90^{\circ}\right.$ at $\left.\omega=3.5\right)$

(2) A maximum low-frequency droop of $-3 \mathrm{~dB}\left(\left|\theta / \theta_{C}\right| \geq-3 \mathrm{~dB}\right.$ for $\left.\omega \leq B W\right)$ and the form of the compensation (or the ratio of $T_{p 1} / T_{p 2}$ ) must minimize resonant peak $\left|\theta / \theta_{c}\right|_{\max }$ :

The relationship between the open-10op transfer function and the closed-10op transfer function has already been stated in defining terminology. This relationship is characteristic of unity feedback systems, such as the one of Figure 2.4. Designing a (pilot) controller for such a system, in this analysis, was greatly facilitated by the use of the Nichols chart.

The Nichols chart, shown in Figure 2.6 with the Neal and Smith standards of performance, is a graph illustrating lines of constant closed-loop amplitude and phase on a grid of open-loop amplitude versus phase. Simply plotting the open-loop $\left(\theta / \theta_{e}\right)$ amplitude versus phase on a Nichols chart gives instantaneous information regarding closed-10op performance. The sample open-10op curve, depicted in Figure 2.6 , is representative of a system correctly compensated to the desired droop, bandwidth and a closed-10op amplitude $\left|\theta / \theta_{c}\right|$ roughly equal to $O \mathrm{~dB}$ at the bandwidth frequency. With this template for low frequency performance, finding the optimal compensation, that is the compensation meeting Neal and Smith's performance standards and minimizing resonance peak $\left|\theta / \theta_{C}\right|_{\max }$ is the remaining task. 


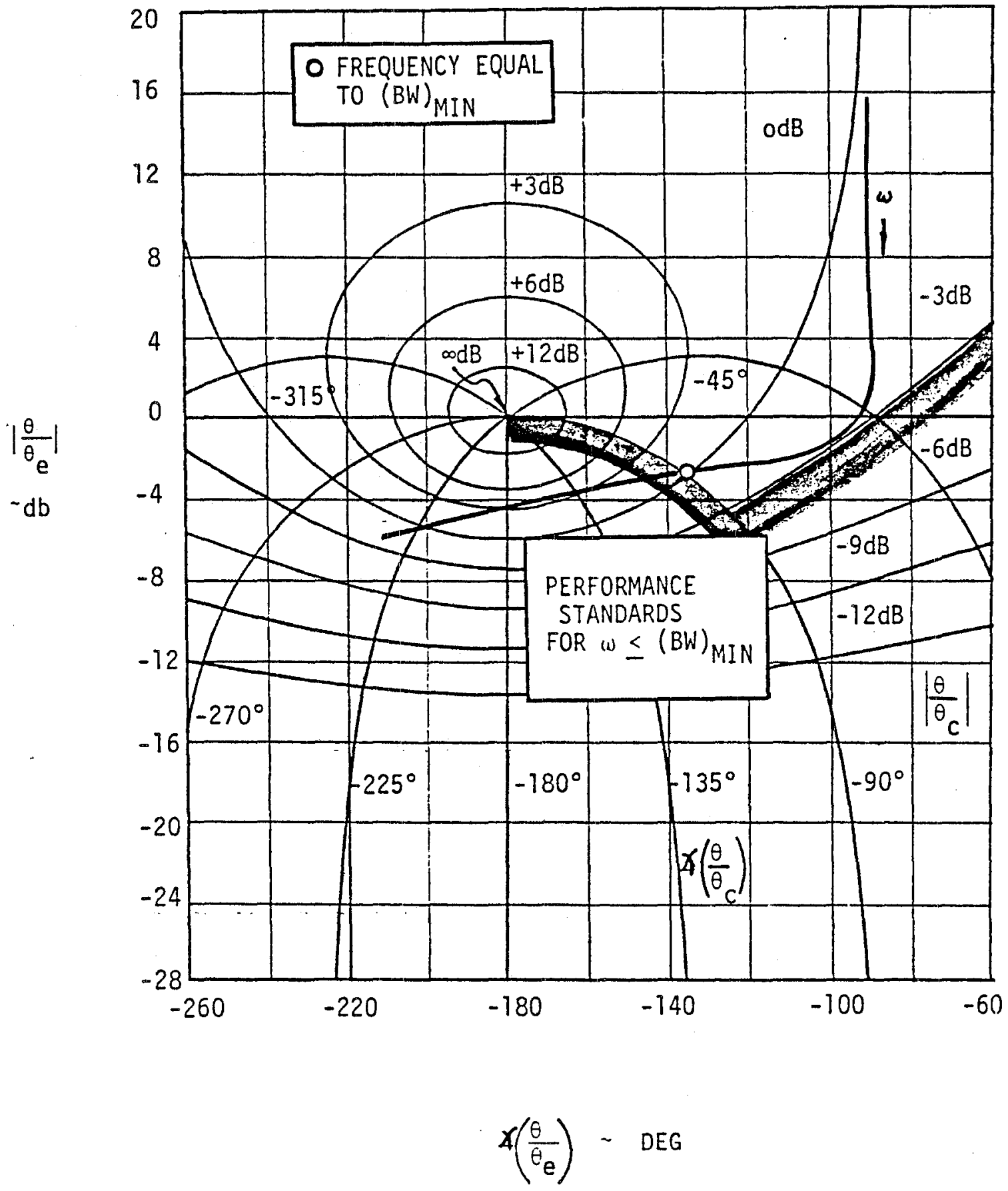

Figure 2.6 Nichols Chart Showing Performance Standards and a Sample AmplitudePhase Curve 
The effect of adding the simplest form of compensation, that is $K_{p}$, the controller gain, slides the amplitude-phase curve vertically on the Nichols chart. Adding the frequency dependent lead-lag compensation is not so simple.

Neal and Smith's argument for their optimal lead-lag compensation is based on two observations; on the Nichols chart, resonance occurs at the point where the amplitude-phase curve is tangent to the loci of constant $\left|\theta / \theta_{c}\right|$. And, an important factor influencing the magnitude of the closed-loop resonance is the slope of the amplitude-phase curve in the vicinity of Bw. Noting the trends and limitations of the lead-lag network to control this slope, Neal and Smith developed an overlay, containing the amplitude-phase curves for the "optimum" pilot compensation. This overlay, shown in Figure 2.7, would produce a slope at $\omega=B W$ conducive to minimizing closed-loop resonance. To illustrate the use of this compensation overlay, consider the following example.

Given the Bode amplitude and phase characteristics of configuration $2 G^{\prime} s$ pitch attitude response, $\left|\theta(j \omega) / F_{s}(j \omega)\right|$ and $\Varangle\left(\theta(j \omega) / F_{s}(j \omega)\right)$ of Figure 2.8, find the pilot parameters that will meet the performance standards and minimize the system's oscillatory tendencies.

The analysis begins by adding the pilot's time delay at some nominal $K_{p}($ say 1.0$)$. See Figure 2.8 .

$$
\begin{aligned}
& \left(\theta / \theta_{\mathrm{e}}\right)^{*}=1.0 \mathrm{e}^{-0.3 \mathrm{~s}}\left(\theta / \mathrm{F}_{\mathrm{s}}\right) \text { or } \\
& \Varangle\left(\theta / \theta_{\mathrm{e}}\right)^{*}=\Varangle\left(\theta / \mathrm{F}_{\mathrm{s}}\right)+57.3(-0.3 \omega) \\
& \left|\theta / \theta_{\mathrm{e}}\right|^{*}=\left|\theta / \mathrm{F}_{\mathrm{s}}\right|
\end{aligned}
$$




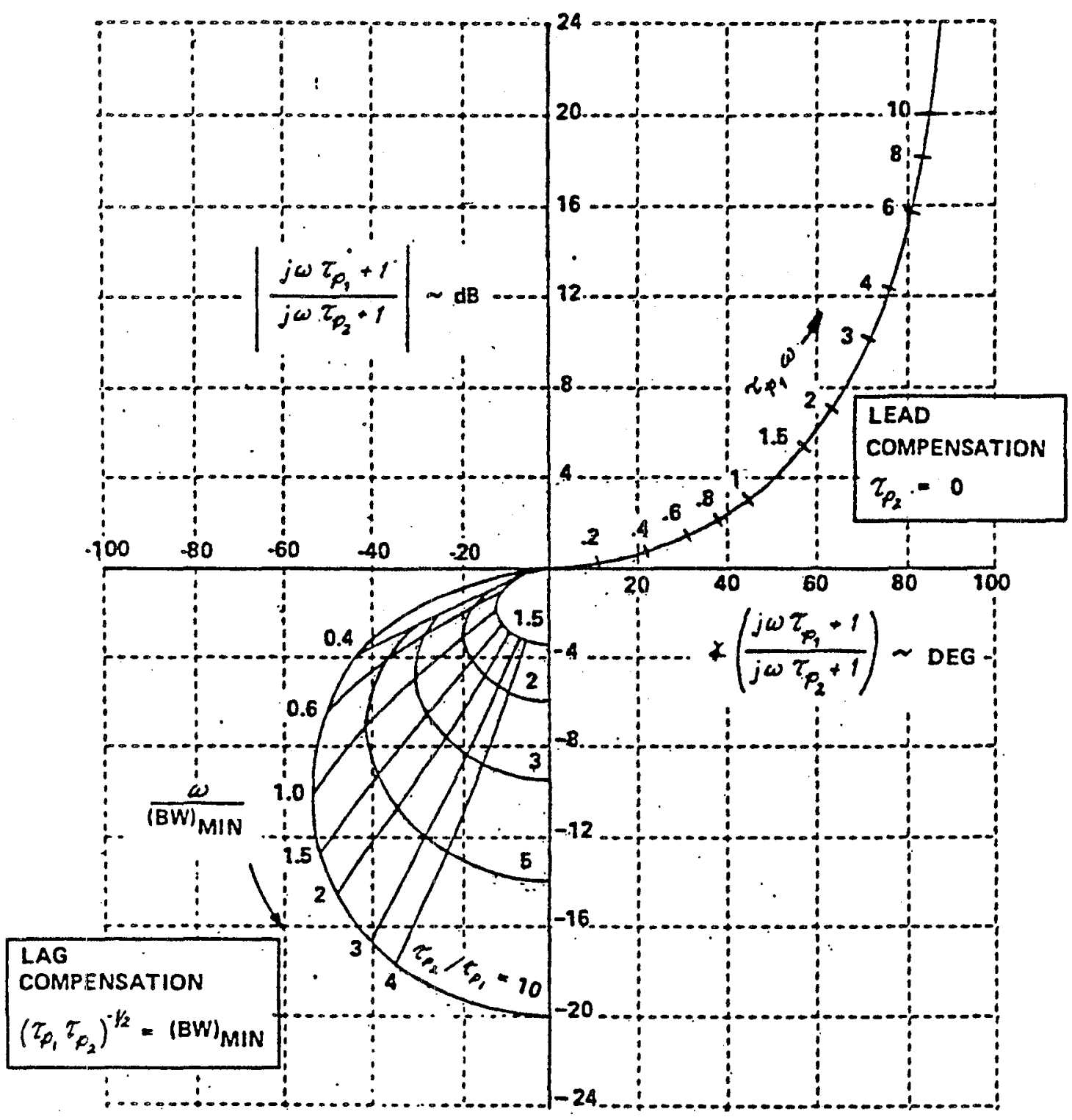

Figure 2.7 Overlay of Ampl itude-Phase Curves for "Optimum" Pilot Compensation 


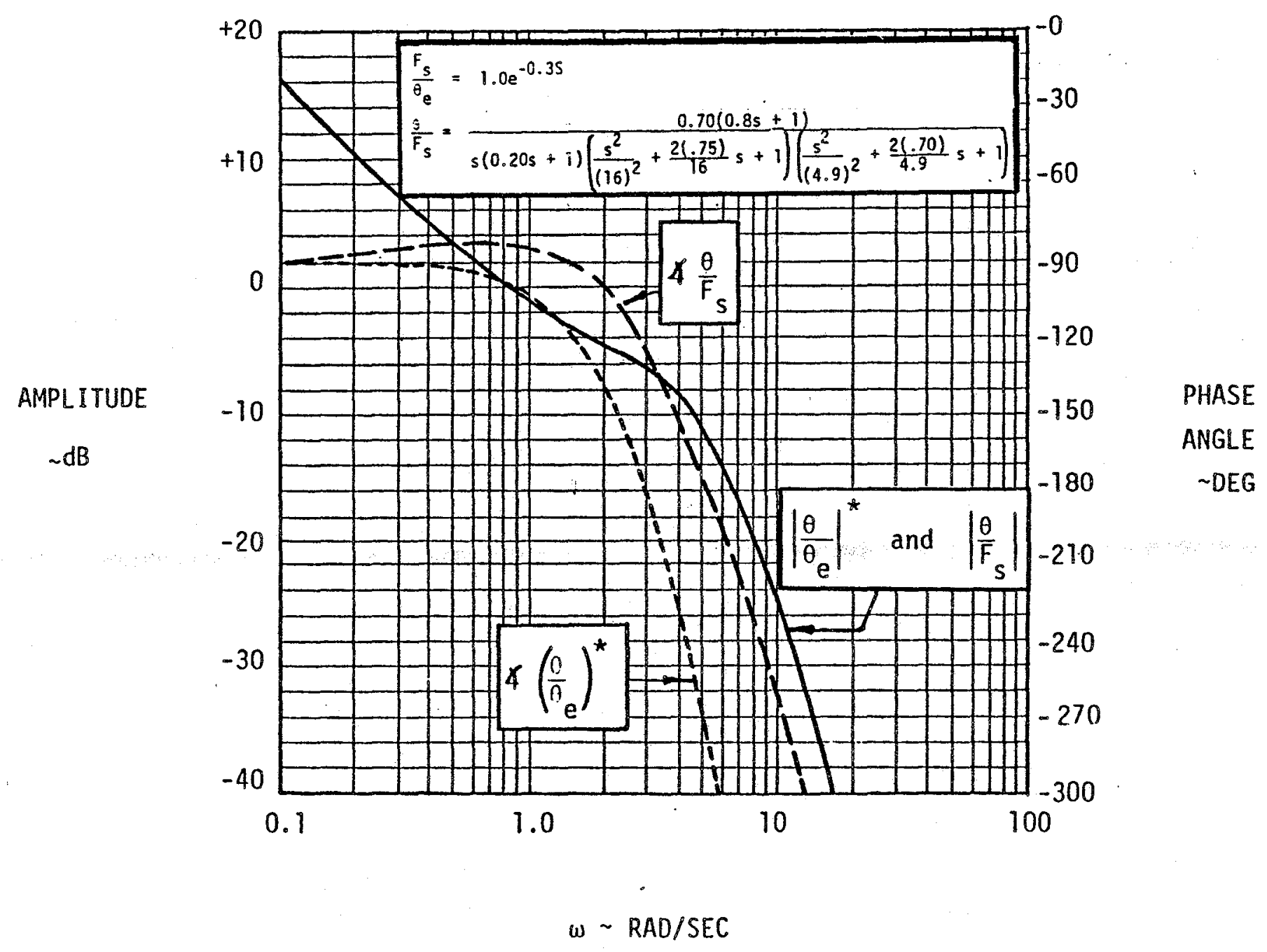

Figure 2.8 Open-Loop Bode Characteristics for Configuration 2G 
Plotting $\left|\theta / \theta_{\mathrm{e}}\right|^{*}$ versus $\Varangle\left(\theta_{0} / \theta_{\mathrm{e}}\right)^{*}$, shown in Figure 2.9, on the Nichols chart and for the moment, neglecting the vertical placement of the amplitude-phase curve, it is obvious that at the desired bandwidth ( $3 \mathrm{rad} / \mathrm{sec}$ in this case), 40 degrees of lead must be added to obtain low frequency performance comparable to the amplitude-phase curve of Figure 2.6. From the overlay, 40 degrees of lead at $\omega=3.0 \mathrm{rad} / \mathrm{sec}$ translates into $T_{p 7^{\omega}}=.85$ or $T_{p 1}=.28$. Setting $T_{p 1}$, the overlay's origin is then centered both horizontaliy and vertically on a number of points on the $\theta / \theta_{e}{ }^{*}$ amplitude-phase curve. The compensation is added graphically by locating the point of the compensation amplitudephase curve corresponding to the value of $0.28 \omega$, where $\omega$ is the frequency corresponding to the point of $\theta / \theta_{e}{ }^{*}$ amplitude-phase curve positioned at the overlay's origin. The compensated curve, depicted as the dashed line in Figure 2.9, must now be positioned vertically to meet the performance standards.

Adding a gain of $2 \mathrm{~dB}$ will shift the compensated curve up to the desired position shown in Figure 2.10. The resulting resonance occurs at $\omega=4.0 \mathrm{rad} / \mathrm{sec}$ and has a magnitude of $7 \mathrm{~dB}$. A few attempts will usually be needed to obtain the compensation that will produce minimum resonance. Neal and Smith found that a lead compensation of $35^{\circ}$ at $\omega=$ BW was suitable to produce a resonant peak of $6 \mathrm{~dB}$, as opposed to the example's first attempt that used $40^{\circ}$ of lead compensation at $\omega=\mathrm{BW}$ for $\left|\theta / \theta_{c}\right|_{\max }=7 \mathrm{~dB}$.

In Figure 2.11, Neal and Smith's:ultimate results are shown that relate pilot rating with the resulting pilot compensation and magnitude of resonance peak. The diagram divides the pilot's rating into three levels of handling qualities. 


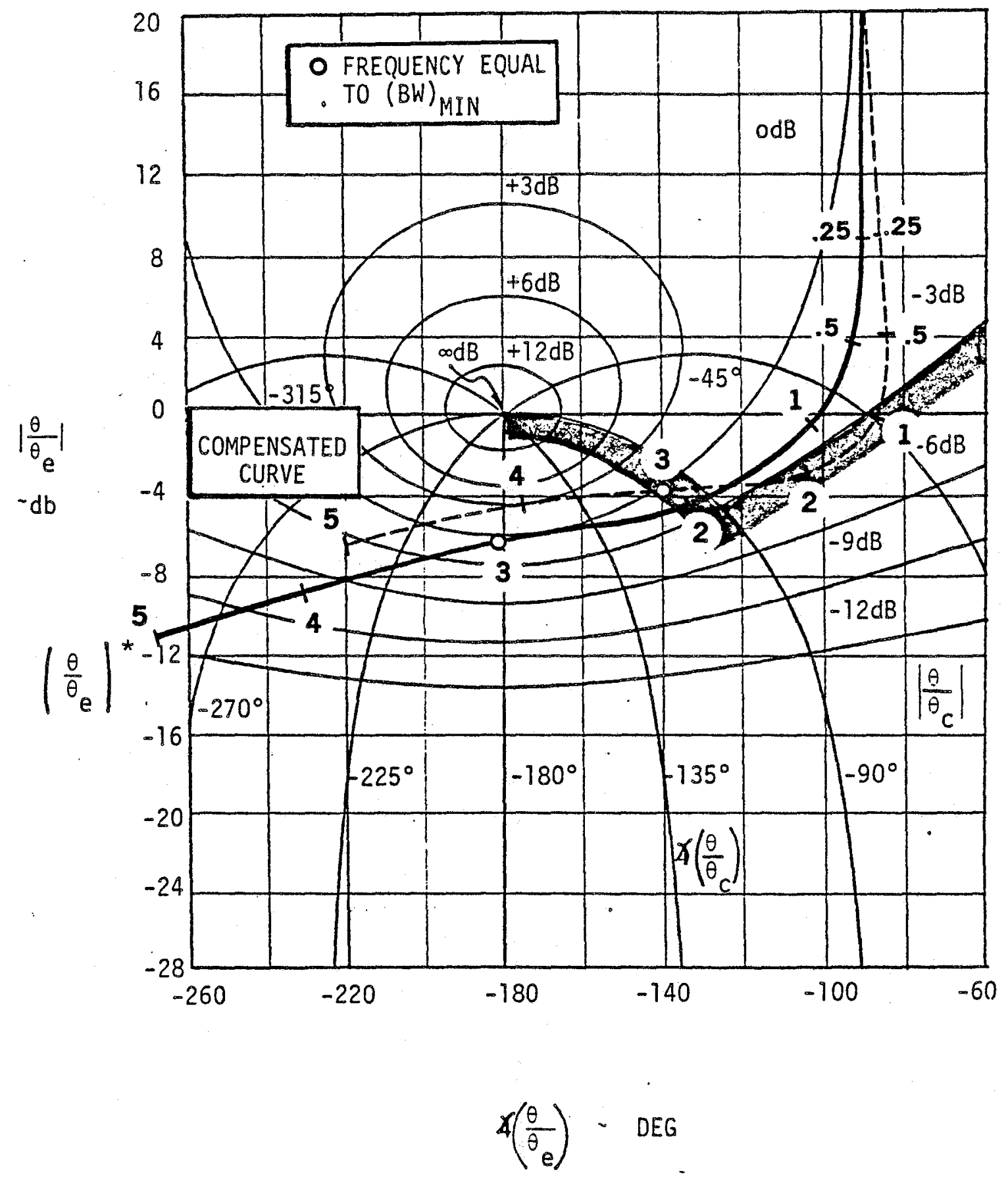

Figure 2.9 Uncompensated/compensated Amplitude-Phase Curve for Configuration $2 G$ 


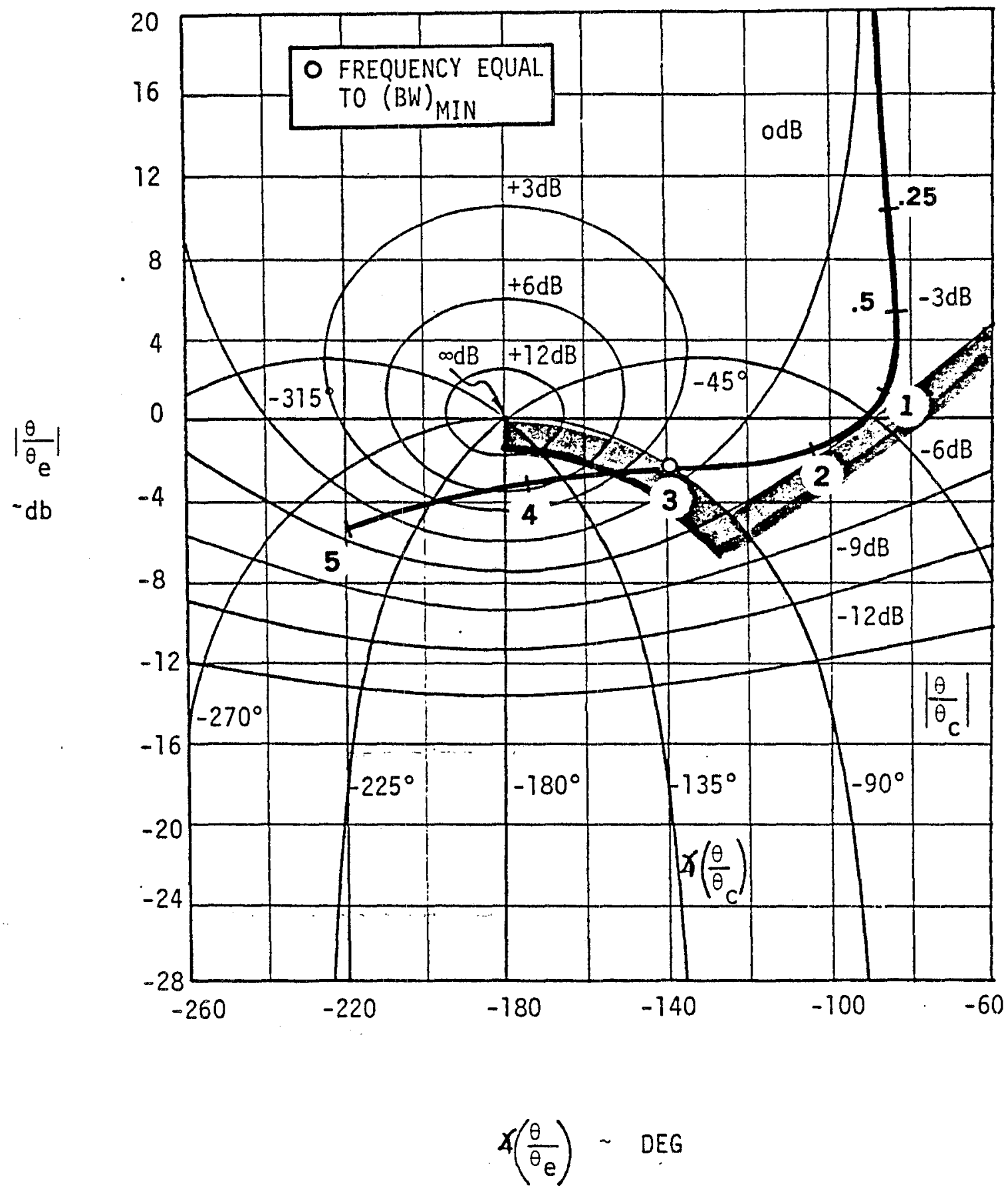

Figure 2.10 Gain-Adjusted; Compensated Amplitude-Phase Curve for Configuration 2G 


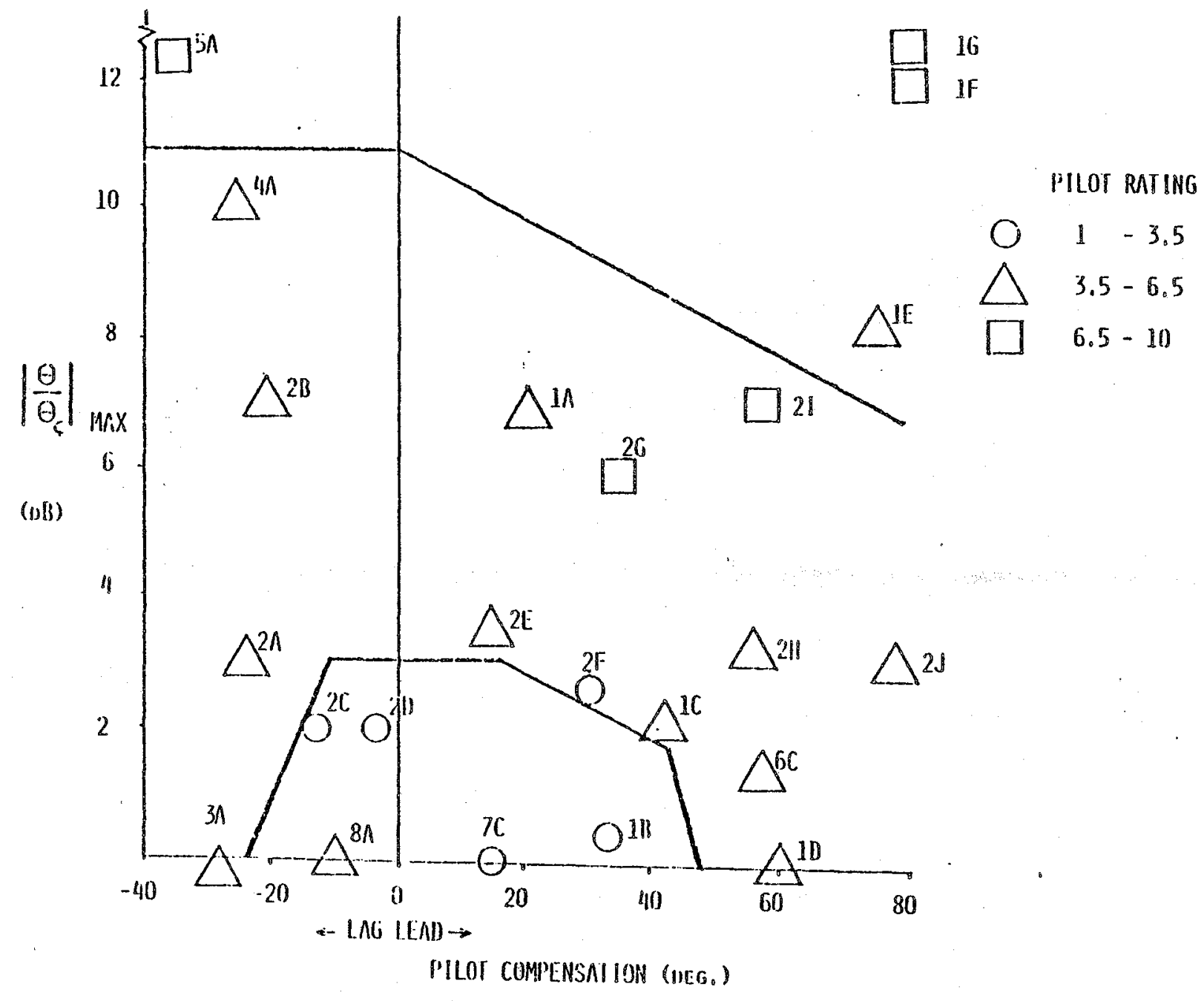

Figure 2.11 Nea -Smith Results 
Cooper-Harper

Level $1 \quad 1.0-3.5 \quad$ good

Level $2 \quad 3.5-6.5$ fair

Level $3 \quad 6.5-10.0$ poor

The dynamics of the configurations represented in Figure 2.11 can be found in Table 2.1. (More were evaluated in Ref. 1)

Overall, the results of this analysis were encouraging, however, problems inherent to pilot parameter selection made this method cumbersome.

One of the biggest problems centered on bandwidth selection. In the experiment, some of the aircraft were flown at different flight conditions (i.e. slower flight speed) and a different bandwidth was used to create the needed correlation. Such was the case in the above example with bandwidth set at $3.0 \mathrm{rad} / \mathrm{sec}$. Bandwidth is also dependent on task and how aggressively the pilot feels he must be to satisfy the task's objectives. In their analysis, Neal and Smith said, "Bw was determined by trying a few values of Bw in the evaluation of a cross section of configurations until the resulting values of $\left|\theta /{ }_{c}\right|_{\max }$ correlated qualitatively with pilot comments concerning PIO tendencies." This fact makes the analysis somewhat impractical as a predictive tool if a priori knowledge of bandwidth is required. In addition, the determination of a suitable pilot representation has always been difficult. Thus an alternate method is desired.

This alternate method will evolve from the optimal control model of the next chapter. The following chapters will approach the NealSmith method using a better model of the human controller. 
CHAPTER 3

THE OPTIMAL CONTROL MODEL

In 1970, Kleinman, Baron, and Levinson ${ }^{[2]}$ published a mathematical model of human response using optimal-control and estimation theory. This optimal-control model (OCM) of the pilot assumes that the welltrained, well-motivated human operator chooses his control input $u_{p}$ subject to human limitations such that the following objective function is minimized

$$
J_{p}=E\left[\lim _{T \rightarrow \infty} \frac{1}{T} \int_{0}^{T}\left(\bar{y}^{\prime} Q \bar{y}+r u_{p}^{2}+g \dot{u}_{p}^{2}\right) d t\right\}
$$

where $g$ is selected to obtain a chosen neuromuscular lag time constant ${ }^{N}$. The pilot's input, the solution to the optimal control problem as stated, is expressed as

$$
\tau_{N} \dot{u}_{p}=-e_{e}^{*-}-u_{p}+v_{m}
$$

with $\dot{\gamma}$, the best state estimate conditioned on delayed, noisy observations, and $e^{*}$, the optimal control gains, and $v_{m}$, the motor noise, qualitatively illustrated in the overall pilot model of Figure 3.1. The model's individual components (i.e., state estimator, predictor, and control law) will be discussed in the coming sections of this chapter.

To aid readers unfamiliar with the $0 \mathrm{CM}$, Chapter 3 is divided into three sections. The first presents Kleinman's solution for the 


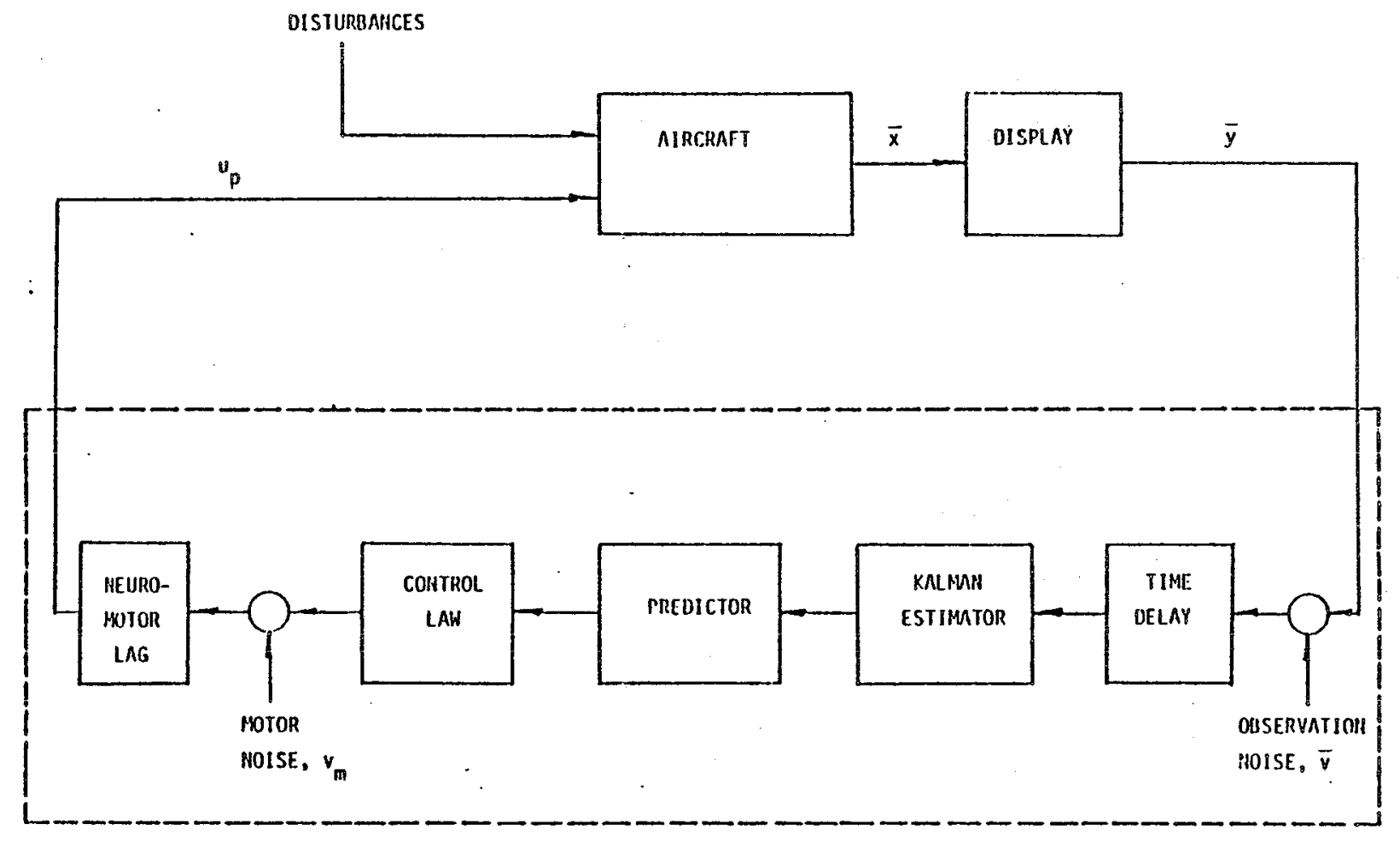

PILOT (OCM)

Figure 3.1 Optimal Control Pilot Model 
optimal control of a linear system with time delay and observation noise, a useful forerunner to the development of the OCM. The second section extends the problem of the first section, incorporating motor noise and neuromuscular lag, thereby establishing the structure of the OCM. Section 3 , utilizing the linear time-invariant equations of the previous sections, forms the OCM's transfer function for the human pilot model, an important relationship needed in the Neal and Smith analysis.

\subsection{Optimal Control of a Linear System with Time Delay and Measurement} Noise

An important problem, basic to the development of the OCM, is the general problem of identifying a controller that will minimize a quadratic cost when measurements consist of a linear combination of corrupted, delayed states. Kleinman ${ }^{[3]}$ has shown for such problems the optimal state estimator is generated by cascading a Kalman Filter and a least-mean-square predictor.

The prescribed problem deals specifically with a given time invariant system presented in the following state space form.

$$
\begin{aligned}
& \dot{\bar{x}}(t)=A \bar{x}(t)+B \bar{u}(t)+\bar{w}(t) \\
& \bar{y}(t)=C \bar{x}(t-\tau)+\bar{v}(t-\tau)
\end{aligned}
$$

The usual conditions of $\{A, B\}$ and $\{A, C\}$ being stabilizabie and detectabie pairs are assumed. Also, $\bar{w}(t)$ and $\bar{v}(t)$ are considered stationary Gaussian white noise vectors with auto-covariance matrices.

$$
\begin{aligned}
& E\left\{\bar{w}(t) \bar{w}^{\prime}(\sigma)\right\}=W \delta(t-\sigma) \\
& E\left\{\bar{v}(t) \bar{v}^{\prime}(\sigma)\right\}=V \delta(t-\sigma)
\end{aligned}
$$


where $W, V>0$. The time delay, $\tau \geq 0$, is fixed in Eq. (3.4). Horeover, the system's initial conditions are assumed to be equal to zero.

The problem's objective is to find the non-anticipative control $\bar{u}$ that minimizes the cost

$$
\begin{aligned}
J(\bar{u})=\lim _{T \rightarrow \infty} \frac{1}{T} E\left\{\int_{\tau}^{T}\right. & {\left[\bar{x}^{\prime}(t-\tau) Q \bar{x}(t-\tau)\right.} \\
& \left.\left.+\bar{u}^{\prime}(t-\tau) R \bar{u}(t-\tau)\right] d t\right\},
\end{aligned}
$$

conditioned on the observed variable $\bar{y}(\sigma), \sigma \leq t$, where $R>0$ and the pair $\{A, \sqrt{Q}\}$ is completely observable, and where the delayed state $\bar{x}(t-\tau)$ is generated by

$$
\dot{\bar{x}}(t-\tau)=A \bar{x}(t-\tau)+B \bar{u}(t-\tau)+\bar{w}(t-\tau) .
$$

The solution is obtained by first defining the least-mean-square estimate of $\bar{x}(t-\tau)$ as

$$
\hat{\bar{x}}(t-\tau) \doteq E\{\bar{x}(t-\tau) \mid \bar{y}(\sigma), \sigma \leq t\}
$$

The estimate $\hat{\bar{x}}(t-\tau)$ may be computed directly from $\bar{y}$, using a Kalman Filter modified to include the deterministic input $\bar{u}(t)$,

$$
\begin{aligned}
& \dot{\hat{\hat{x}}}(t-\tau)=A \hat{\bar{x}}(t-\tau)+\Sigma(t-\tau) C^{\prime} V^{-1}[\bar{y}(t)-C \hat{\bar{x}}(t-\tau)] \\
& +\quad+B \bar{u}(t-\tau) \\
& \hat{\vec{x}}(0)=0
\end{aligned}
$$

where $\Sigma(t)$ is the covariance of the Kalman Filter estimation error, $\bar{e}(t)=\bar{x}(t)-\hat{\bar{x}}(t) . \quad \Sigma(t)$ is also the positive definite solution of

$$
\begin{aligned}
& \dot{\Sigma}(t)=A \Sigma(t)+\Sigma(t) A^{\prime}+W-\Sigma(t) C^{\prime} V^{-1} C \Sigma(t) \\
& \Sigma(0)=0
\end{aligned}
$$


As time tends to infinity, $\Sigma(t)$ approaches the constant, steady-state solution, $\bar{\Sigma}$, a unique positive definite matrix. Substituting $\bar{\Sigma}$ for $\Sigma(t-\tau)$ in Eq. (3.9) produces the desired steady-state Kalman Filter. Unfortunately, given the current observation, $\tilde{y}(t)$, the Kalman Filter produces only delayed estimate $\hat{\hat{x}}(t-\tau)$ while the system, with control, is at the current state $\bar{x}(t)$. In order to minimize $J$, the optimal control must be generated from $\hat{\bar{x}}(t-\tau)$, not $\hat{\bar{x}}(t)$ as in the standard linear, quadratic, Gaussian (LQG) optimal control approach. This dilemma is resolved by the development of a least-mean-square predictor capable of generating an estimate of $\hat{\bar{x}}(t)$ from the delayed estimate $\hat{\bar{x}}(t-\tau)$. The advantage of obtaining the predicted state reduces the problem to one equivalent to the well-known LQG problem using a modified, but equivalent cost function.

Two basic concepts with parallel interpretations in the Kalman Filter and the least-mean-squared predictor will pormit the needed modification of the cost functional. First, the least-mean-square estimator (predictor) provides an orthogonal projection of $\bar{x}(t)$ $(\hat{\bar{x}}(t))$ onto the subspace of the observed variable $\bar{y}(\hat{\bar{x}}(t-\tau))$, leading to an extremely useful property; the estimate $\hat{\bar{x}}$ (prediction $\bar{\gamma}$ ) is orthogonal to its error $\bar{e}\left(\bar{e}_{p}\right)$ as shown in Fig. 3.2a(3.2b). The second important task, establishing the independence of the estimation (prediction) error from the deterministic input $\bar{u}$, wi 17 become evident upon finding the differential equations generating the estimation (prediction) error.

The solution proceeds by first investigating the estimation error, $\ddot{e}(t)$. To generate $\bar{e}(t)$, substitute Eq. (3.4) into Eq. (3.9). 


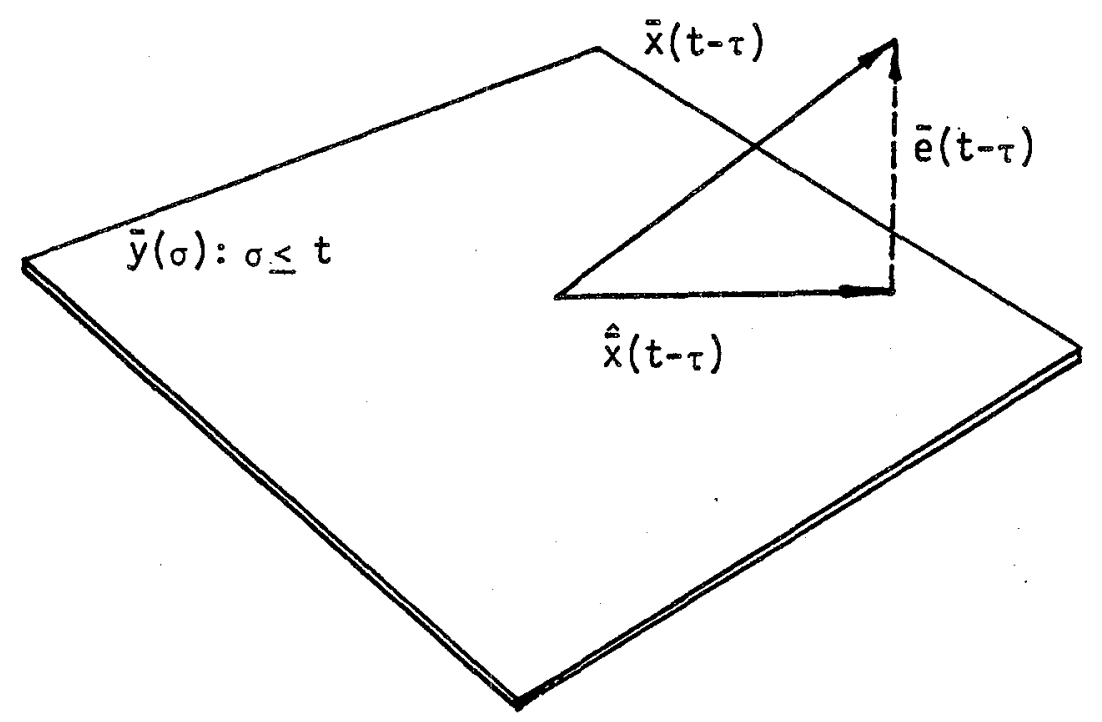

Figure 3.2a Estimator Orthogonally Projects the Delayed State onto the Subspace of Observations

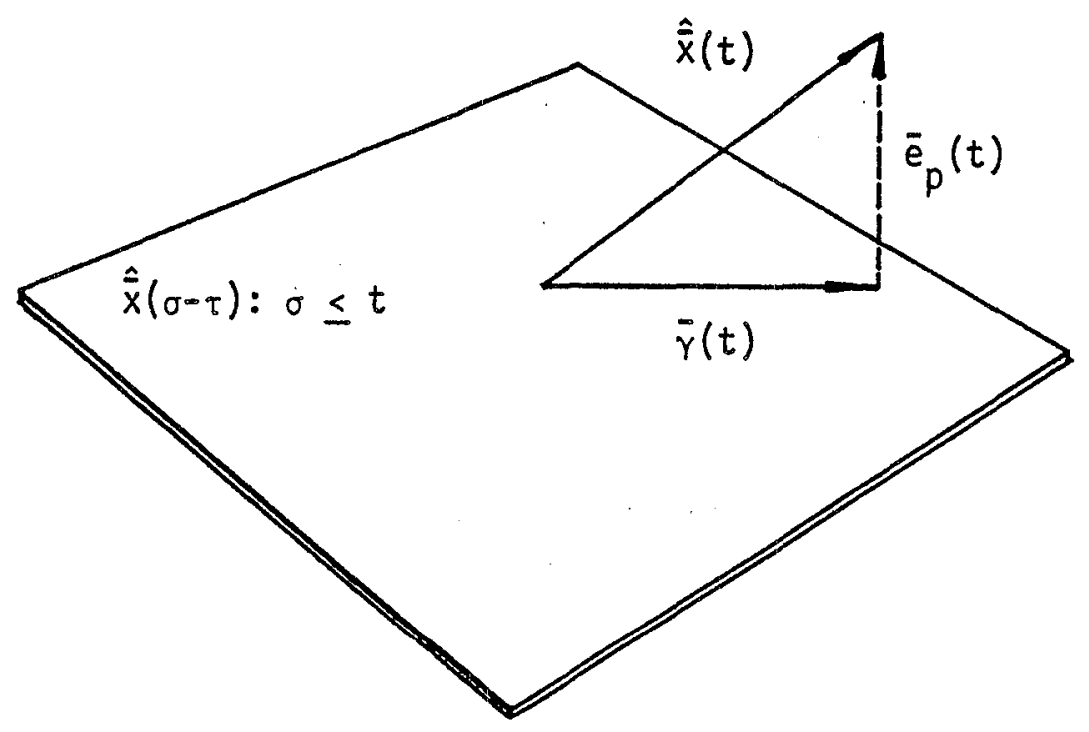

Figure 3.2b Predictor Orthogonally Projects the Current Estimate onto the Subspace of Delayed Estimates 


$$
\begin{aligned}
& \dot{\bar{x}}(t-\tau)=A \hat{\bar{x}}(t-\tau)+\bar{\Sigma} C^{\prime} V^{-1} C[\bar{x}(t-\tau) \\
& -\hat{\bar{x}}(t-\tau)]+B \bar{u}(t-\tau)+\bar{\Sigma} C^{\prime} V^{-1} \bar{v}(t-\tau)
\end{aligned}
$$

Since all time-dependent variables are at the same point in time, setting $t=t-\tau$ will provide an equivalent form of Eq. (3.11). Then, noting that the error rate may be expressed as

$$
\dot{\bar{e}}(t)=\dot{\bar{x}}(t)-\dot{\bar{x}}(t) \text {, }
$$

substitute Eq. (3.3) and the equivalent form of Eq. (3.11) into Eq. (3.12)

$$
\begin{gathered}
\dot{e}(t)=\left[A-\bar{\Sigma} C^{\prime} V^{-1} C\right] \bar{e}(t)-\bar{\Sigma} C^{\prime} V^{-1} \bar{v}(t) \\
+\bar{w}(t)
\end{gathered}
$$

Clearly, the error is independent of the input $\bar{u}(t)$. Eq. (3.13) will further aid in making the first of two modifications to the cost, $\mathrm{J}$. The second modification will follow the development of the predictor, proceeding roughly along the same line of reasoning as the first.

To modify the cost, J, start by rewriting the first term of Eq. (3.6) as

$$
\begin{gathered}
E\left\{\bar{x}^{\prime}(t-\tau) Q \bar{x}(t-\tau)\right\}=E\left\{\bar{e}^{\prime}(t-\tau) Q \bar{Q}(t-\tau)\right\} \\
+E\left\{\hat{\bar{x}}^{\prime}(t-\tau) Q \hat{\bar{x}}(t-\tau)\right\}
\end{gathered}
$$

since $E\left\{\hat{\bar{x}}^{\prime}(t-\tau) \bar{e}(t-\tau)\right\}=0$ when $\hat{\bar{x}}(t-\tau)$ is a least-mean-square estimate. Substituting Eq. (3.14) into Eq. (3.6) transforms the original cost function into

$$
J(\bar{u})=\lim _{T \rightarrow \infty} \frac{1}{T} E\left\{\int_{\tau}^{T} \bar{e}^{\prime}(t-\tau) Q \bar{e}(t-\tau) d t\right\}+J_{1}(\bar{u})
$$


where

$$
J_{\eta}(\bar{u})=\lim _{T \rightarrow \infty} \frac{1}{T} E\left\{\int_{\tau}^{T}\left[\hat{\bar{x}}^{\prime}(t-\tau) Q \hat{\bar{x}}(t-\tau)+\bar{u}^{\prime}(t-\tau) \operatorname{Ru}(t-\tau)\right] d t\right\}
$$

From previous results, the error is independent of $\bar{u}(t)$, making the first term of Eq. (3.15) unaffected by the choice of $\bar{u}$. Hence, the original problem can be equivalently restated as finding the control input $\bar{u}(t)$ that minimizes $J_{1}(\bar{u})$, with $\hat{\bar{x}}(t-\tau)$ generated by

$$
\begin{aligned}
\dot{\hat{x}}(t-\tau)=A \hat{\bar{x}}(t-\tau) & +B \bar{u}(t-\tau) \\
& +\bar{\Sigma} C^{\prime} V^{-1}[C \bar{e}(t-\tau)+\bar{v}(t-\tau)]
\end{aligned}
$$

in the steady state.

Wonham ${ }^{[4]}$ has shown that the process, $\bar{\Sigma} C^{\prime} V^{-1}[C \bar{e}(t-\tau)+\bar{v}(t-\tau)]$, can be represented as a white noise process $\tilde{\bar{W}}(t-\tau)$ whose autocovariance matrix is given by

$$
\begin{aligned}
E\left\{\tilde{\bar{w}}(t) \tilde{\bar{W}}{ }^{\prime}(\sigma)\right\} & =\bar{\Sigma} C^{\prime} V^{-1} C \bar{\Sigma} \delta(t-\sigma) \\
& =\tilde{W} \delta(t-\sigma)
\end{aligned}
$$

This equivalent process simplifies the analysis of $\hat{\bar{x}}(t-\tau)$ by restating Eq. (3.17) as

$$
\dot{\hat{x}}(t-\tau)=A \hat{\bar{x}}(t-\tau)+B \bar{u}(t-\tau)+\tilde{\bar{w}}(t-\tau)
$$

Note that Eq. (3.19), representing the new system to be regulated, governs only the delayed estimate, $\hat{\bar{x}}(t-\tau)$. Now, the original problem is reduced to finding the nonanticipative $\bar{u}(t)$ that will minimize $J_{j}(\bar{u})$. The solution will be realized by first finding the predicted state $\bar{\gamma}(t)$ based on the output, 


$$
\bar{y}(\sigma)=\{\hat{\tilde{x}}(\sigma-\tau) ; \quad \sigma \leq t\}
$$

Kleinman defined the least mean-square predicted state, $\bar{\gamma}(t)$, as

$$
\ddot{\gamma}(t) \doteq E\{\hat{\bar{x}}(t) \mid \bar{y}(\sigma), \sigma \leq t\}
$$

conditioned on the output of the Kalman Filter. To generate $\gamma(t)$, start by rewriting Eq. (3.19) as

$$
\dot{\bar{y}}(t)=A \bar{y}(t)+B \bar{u}(t-\tau)+\tilde{\bar{w}}(t-\tau)
$$

Since the system is linear and $\bar{u}(t-\tau)$ is deterministic, the output of the Kalman Filter, $\bar{y}(t)=\hat{\bar{x}}(t-\tau)$, may be decomposed into two parts: $\bar{y}_{u}(t)$, or that due to the deterministic input, and $\bar{r}(t)$, or that generated by the process noise $\tilde{\bar{w}}(t-\tau)$. Splitting Eq. (3.22) apart according to input results in

$$
\dot{\bar{y}}_{u}(t)=A \bar{y}_{u}(t)+B \bar{u}(t-\tau)
$$

and

$$
\dot{\tilde{r}}(t)=A \bar{r}(t)+\tilde{\tilde{w}}(t-\tau)
$$

where

$$
\bar{y}(t)=\bar{r}(t)+\bar{y}_{u}(t)
$$

and

$$
\hat{\bar{x}}(t)=\tilde{r}(t+\tau)+\bar{y}_{u}(t+\tau)
$$

Note that the theoretical $\hat{\bar{x}}(t)$ is actually an unattainable current state estimate; attainable only if either the time delay or the observation noise is absent. With Eq. (3.26) and the fact that $\bar{y}_{u}$ is a deterministic vector, simplify the definition of the predicted state, Eq. (3.21), to

$$
\bar{\gamma}(t)=\bar{y}_{u}(t+\tau)+E\{\bar{r}(t+\tau) \mid \bar{y}(\sigma), \quad \sigma \leq t\}
$$


Eq. (3.24) shows the independence of $\bar{r}(t)$ from the deterministic $\bar{y}_{u}(t)$, automaticaliy reducing the second term of Eq. (3.27) to $E\{\bar{r}(t+\tau) \mid \bar{r}(0)$, $\sigma \leq t\}$. To evaluate this expression further, write the solution of Eq. (3.24) as

$$
\bar{r}(t+\tau)=e^{A \tau} \bar{r}(t)+\int_{t}^{t+\tau} e^{A(t+\tau-\sigma)} \tilde{\bar{w}}(\sigma-\tau) d \sigma .
$$

Remember, $\tilde{\bar{w}}(t)$ is a zero-mean white noise process, thus

$$
E\{\bar{r}(t+\tau) \mid \bar{r}(\sigma), \sigma \leq t\}=e^{A \tau} \bar{r}(t),
$$

thereby, simplifying the predicted state, $\bar{\gamma}$, to

$$
\bar{\gamma}(t)=\bar{y}_{u}(t+\tau)+e^{A \tau_{\bar{r}}(t)}
$$

Analytically, a differential equation generating $\bar{\gamma}$ would be preferred. Differentiating Eq. (3.30) and then, substituting Eq. (3.23) and Eq. (3.24) with Eq. (3.25) into the expression for $\bar{\gamma}(t)$, gives the desired differential equation

$$
\dot{\bar{\gamma}}(t)=A \bar{y}_{u}(t+\tau)+B \bar{u}(t)+e^{A \tau}(A \bar{r}(t)+\tilde{\bar{w}}(t-\tau))
$$

or

$$
\dot{\bar{\gamma}}(t)=A \bar{\gamma}(t)+B \bar{u}(t)+e^{A \tau} \tilde{w}(t-\tau)
$$

with an equivalent representation obtained by substituting Eq. (3.25) into Eq. (3.30) or

$$
\bar{\gamma}(t)=\bar{\xi}(t)+e^{A \tau}(\hat{\bar{x}}(t-\tau)-\bar{\xi}(t-\tau))
$$

where

$$
\dot{\bar{\xi}}(t)=A \bar{\xi}(t)+B \bar{u}(t)
$$


Eqns. (3.33), formulated first by Kleinman[3], govern the controller's prediction process. Later, Eq. (3.32) will be instrumental in developing closed-form solutions for both the state covariance matrix and the total cost function.

But to continue, a control $\bar{u}$ must be found to minimize $\mathrm{J}$ or equivalentiy, to minimize $J_{7}$. Note that so far, expressions have been developed to produce a state estimate at the current time, $\bar{\gamma}(t)$. Now the cost $J_{1}$ (Eqn. 3.16) must be rewritten in terms of $\bar{\gamma}_{0}$ To begin, express $J_{l}$ as

$$
J_{q}(\bar{u})=\lim _{T \rightarrow \infty} \frac{T}{T} E\left\{\int_{0}^{T}\left[\hat{\bar{x}}^{\prime}(t) Q \hat{\bar{x}}(t)+\bar{u}^{\prime}(t) R \bar{u}(t)\right] d t\right\}
$$

Paralleling the development of $j_{1}$, decompose the unattainable current estimate $\hat{\bar{x}}(t)$ into its orthogonal components: $\bar{\gamma}_{9}$ the orthogonal prow jection of $\hat{\bar{x}}(t)$ onto the subspace of the delayed estimate $\hat{\bar{x}}(t-\tau)$, and $\mathrm{e}_{p}$, the prediction error defined as

$$
e_{p}(t) \doteq \hat{\bar{x}}(t)-\vec{\gamma}(t)
$$

Using the same arguments cited in the estimation process, the first term of $J_{1}$ expands to

$$
E\left\{\hat{\bar{x}}^{\prime} Q \hat{\bar{x}}\right\}=E\left\{\bar{e}_{p}^{\prime} Q_{\bar{e}}\right\}+E\left\{\bar{\gamma}^{\prime} Q \ddot{\gamma}^{\prime}\right\}
$$

Upon investigation of Eq. (3.26) and Eq. (3.30), the prediction error, $\vec{e}_{p}$, may be rewritten as $\bar{r}\left(t+_{\tau}\right)=e^{A_{\tau}} \bar{r}(t)$, where $\bar{r}(t+\tau)$ is. given by Eq. (3.28)

$$
\bar{e}_{p}(t)=\int_{t}^{t+\tau} e^{A(t+\tau-\sigma)} \overline{\tilde{w}}(\sigma-\tau) \cdot d \sigma
$$


Letting $\xi=t+\tau-\sigma$ with $d \xi=-d \sigma$,

$$
\bar{e}_{p}(t)=\int_{0}^{\tau} e^{A \xi \tilde{w}(t-\tau) d \xi}
$$

provides the means of evaluating $E\left\{\bar{e}_{p}{ }^{\prime} Q \bar{e}_{p}\right\}$.

$$
\begin{aligned}
E\left\{\bar{e}_{p}^{\prime} \cdot Q \bar{e}_{p}\right\} & =\operatorname{tr}\left(Q E\left\{\bar{e}_{p} \bar{e}_{p}^{\prime}\right\}\right) \\
& =\operatorname{tr}\left(Q \int_{0}^{\tau} e^{A \xi} \tilde{W} e^{A^{\prime} \xi} d \xi\right)
\end{aligned}
$$

Observe that this covariance of the prediction error is also independent

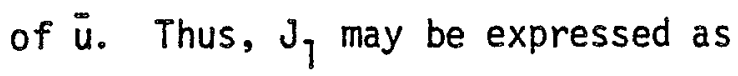

$$
\begin{aligned}
J_{1}(\bar{u})= & \left.\underset{T \rightarrow \infty}{E\left\{\prod_{T \rightarrow \infty}\right.} \frac{1}{T} \int_{0}^{T}\left(\tilde{e}_{p}^{\prime} Q \bar{e} p+\bar{\gamma}^{\prime} Q \bar{\gamma}+\bar{u}^{\prime} R \bar{u}\right) d t\right\} \\
= & \operatorname{tr}\left(Q \int_{0}^{T} e^{A \xi} \tilde{W} e^{A^{\prime} \xi} d \xi\right) \\
& \left.+\underset{T \rightarrow \infty}{E\{i m} \frac{1}{T} \int_{0}^{T} \cdot\left(\bar{\gamma}^{\prime} Q \bar{\gamma}+\bar{u}^{\prime} R \bar{u}\right) d t\right\}
\end{aligned}
$$

And, since the first term of Eq. (3.40) is independent of $\bar{u}$, finding a control to minimize $J_{1}(\bar{u})$ is equivalent to finding a control to minimize

$$
J_{2}(\bar{u})=E\left\{\lim _{T \rightarrow \infty} \frac{1}{T} \int_{0}^{T}\left(\bar{\gamma}^{\prime} Q \bar{\gamma}+\bar{u}^{\prime} R \bar{u}\right) d t\right\}
$$

Finally, the problem is now reduced to the familiar LQG format with $\bar{\gamma}$ governed by Eq. (3.32), or

$$
\dot{\bar{\gamma}}(t)=A \bar{\gamma}(t)+B \bar{u}(t)+\tilde{\bar{w}}_{2}(t)
$$


where

$$
\tilde{\bar{w}}_{2}(t)=e^{A \tau} \tilde{w}(t-\tau)
$$

and

$$
\tilde{W}_{2}=E\left\{\tilde{\bar{W}}_{2} \tilde{\bar{W}}_{2}^{\prime}\right\}=e^{A \tau} \tilde{W}^{A^{\prime} \tau} .
$$

The solution now, is identical to the control $\bar{u}$ that will minimize $\mathrm{J}_{2}$ if all states were available for feedback. The solution [3] is

$$
\bar{u}^{*}(t)=-L^{*} \gamma(t)=-R^{-1} B^{\prime} K_{\gamma}^{\infty}(t)
$$

with $K$, the unique positive definite solution of the algebraic Ricatti matrix equation.

$$
0=K A+A^{\prime} K+Q-K B R^{-1} B^{\prime} K \text {. }
$$

The resulting minimum cost $J_{2}$ is easily evaluated as

$$
J_{2}(\bar{u})=\operatorname{tr}\left(K \tilde{W}_{2}\right)=\operatorname{tr}\left(K e^{A \tau} \tilde{W} e^{A^{8} \tau}\right)
$$

This expression, however, does not represent the total cost.

To evaluate the total cost, we have

$$
J(\bar{u})=\lim _{T \rightarrow \infty} \frac{1}{T} E\left\{\int_{0}^{T} \bar{e}^{\prime}(t) Q \bar{e}(t) d t\right\}+J_{j}(\bar{u})
$$

and

$$
J_{1}(\bar{u})=\operatorname{tr}\left(Q \int_{0}^{\tau} e^{A \xi} \tilde{w} e^{A^{\prime} \xi} d \xi\right)+J_{2}(\bar{u})
$$

Utilizing the previousiy defined relationships

$$
\begin{aligned}
& \tilde{W} \doteq \bar{\Sigma} C^{\prime} V^{-1} C \bar{\Sigma} \\
& \quad \text { and } \\
& \bar{\Sigma} \doteq E\left\{\bar{e}(t) \bar{e}^{\prime}(t)\right\} \text { as } t \rightarrow \infty,
\end{aligned}
$$


we assemble the total cost

$$
\begin{aligned}
J(\bar{u}) & =\operatorname{tr}(Q \bar{\Sigma})+\operatorname{tr}\left(Q \int_{0}^{\tau} e^{A \xi} \bar{\Sigma} C^{\prime} V^{{ }^{-}}{ }^{-1} C \bar{\Sigma} e^{A^{\prime} \xi} d \xi\right) \\
& +\operatorname{tr}\left(K e^{A \tau} \bar{\Sigma} C^{\prime} V^{-1} C \bar{\Sigma} e^{A^{\prime} \tau}\right)
\end{aligned}
$$

or using the techniques of Appendix $A_{3} J(\bar{u})$ is expressible $e^{[3]}$ as

$$
\begin{aligned}
J\left(\bar{u}^{*}\right) & =\operatorname{tr}\left(Q \int_{0}^{\tau} e^{A \xi} W e^{A^{\prime} \xi} d \xi\right)+ \\
& \operatorname{tr}\left(K e^{A \tau} W e^{A^{\prime} \tau}\right)+\operatorname{tr}\left(L_{e} \bar{\Sigma} L_{e}^{\prime}\right) .
\end{aligned}
$$

where

$$
L_{e}=R^{\frac{1}{2}} L^{*} e^{A \tau}
$$

Kleinman remarked that the total cost is composed of three elements, respectively, evolving from the prediction error, the dynamic disturbance $\bar{w}(t)$, and the observation noise $\bar{v}(t)$. In each element, the effect of time delay is evident.

To correlate results between the experiment and the model, state and output statistics are needed. These measures are attainable from the state covariance matrix. Using techniques created to find the total cost, Appendix B formulates the following closed-form expression $^{[3]}$ for the state covariance matrix

$$
\begin{gathered}
E\left\{\bar{x}(t) \bar{x}^{\prime}(t)\right\}=e^{A \tau} \bar{\Sigma} e^{A^{\prime} \tau}+\int_{0}^{\tau} e^{A \xi} W e^{A^{\prime} \xi} d \xi \\
+\int_{0}^{\infty}\left(e^{A \sigma} e^{A \tau} \bar{\Sigma} C^{\prime} V^{-1} C \bar{\Sigma} e^{A^{\prime} \tau} e^{\bar{A}^{\prime} \sigma}\right) d \sigma
\end{gathered}
$$

where

$$
\bar{A}=A-B L^{*}
$$


This section has summarized Kleinman's controller that will minimize a given quadratic objective function when the measurements consist of a linear combination of delayed states, corrupted by noise. Furthermore, the section concluded with the statement of closed form expressions for the total cost and the state covariance matrix, useful information in gauging a system's performance and a model's validity. The next section will extend the model's structure, incorporating the philosophy of the human controller to develop an optimal-control pilot mode?.

\subsection{The Optimal Control Pilot Model}

Obviously, several parallels exist between the controller of the previous section and the desired model of the human controller. In each, the plant and display dynamics are assumed to be accurately modelled by the time invariant form

$$
\dot{\bar{x}}(t)=A \bar{x}(t)+b u_{p}(t)+\bar{w}(t)
$$

But, in the pilot model, $\bar{x}(t)$ represents the vehicle states, $u_{p}(t)$ is the pilots input, and $\bar{w}(t)$ represents random external disturbances. The time delay, previously associated with the system output, is now internal to the controller. Hence, the pilot observes the following displayed information

$$
\bar{y}(t)=C \bar{x}(t)
$$

but in reality, he perceives

$$
\bar{y}_{p}(t)=c \bar{x}(t-\tau)+\bar{v}(t-\tau)
$$


a delayed, noisy replica of the system's output. Similar to the previous development, the pilot's estimation process may be represented by a Kalman filter cascaded with a least mean-square predictor.

Perhaps one of the biggest differences between the controller of Section 3.1 and the optimal human controller, however, is the addition of neuromuscular dynamics. But in general, the optimal human controller is a natural extension of the previous development.

Consider the task-oriented objective function of the $\mathrm{OCM}$,

$$
J\left(u_{p}^{*}\right)=\lim _{T \rightarrow \infty} \frac{1}{T} E\left\{\int_{0}^{T}\left(\bar{x}^{\prime} Q \bar{x}+r u_{p}^{2}+g \dot{u}_{p}^{2}\right) d t\right\}^{\prime}
$$

conditioned on the perceived $\bar{y}_{p}(\sigma), \sigma \leq t$, with $Q=\operatorname{diag}\left\{q_{1}, q_{2} \ldots\right.$ $\left.q_{n}\right\} ; q_{j} \geq 0$, and $r \geq 0$ and $g>0$, scalar constants. Expanding the objective function to minimize control rate automaticaliy adds a first order lag (or "neuromuscular" dynamics) to the controller. And, since a one-to-one correspondence exists between $g$ and $\tau_{N}$, the researcher has the flexibility to select whatever neuromuscular time constant he desires, by simply varying the weighting on control rate.

To accommodate the neuromotor dynamics or equivalentiy, the expanded cost functional, define a new $(n+1)$ state vector, $\bar{x}^{\prime}(t)=$ $\left\{\bar{x}^{\prime}(t), u_{p}(t)\right\}$, generated by

$$
\begin{aligned}
\dot{\bar{x}}(t) & =A_{0} \bar{x}(t)+b_{0} \mu(t)+\bar{w}_{0}(t) \\
\bar{y}_{p}(t) & =c_{0} \bar{x}(t-\tau)+\bar{v}(t-\tau)
\end{aligned}
$$

with

$$
A_{0}=\left[\begin{array}{l|l}
A & b \\
0 & 0
\end{array}\right] \quad b_{0}=\left[\begin{array}{l}
0 \\
1
\end{array}\right] \quad c_{0}=\left[\begin{array}{lll}
c & 1 & 0
\end{array}\right]
$$


and with $\mu(t) \stackrel{\circ}{=} \dot{u}_{p}(t)$ and $\bar{w}_{0}^{\prime}(t)=\left(\bar{w}^{\prime}(t), 0\right)$.

The optimal control law, assuming full-state feedback, follows directly

$$
\mu^{*}(t)=-2 \dot{\gamma}(t)
$$

with $\bar{r}(t)$, the best estimate of the current state given the condition of the observed measurements. Also we have $(n+1)$ feedback gains, generated from

$$
l=\frac{l}{g} b_{0}^{\prime} k_{0}
$$

with $K_{0}$, the unique positive definite matrix satisfying the $(n+1)$ dimensional Ricatti equation

$$
A_{0}^{\prime} K_{0}+K_{0} A_{0}+Q_{0}-K_{0} b_{0} b_{0}{ }^{\prime} K_{0} / g=0
$$

Finally, $Q_{0}$ is the new weighting matrix, defined as

$$
Q_{0}=\left[\begin{array}{l|l}
Q & 0 \\
\hline 0 & r
\end{array}\right]
$$

The expanded version of the full-state feedback control law ${ }^{[2]}$ is

$$
\mu^{*}(t)=\dot{u}_{p}(t)=-\ell_{n+1} u_{p}(t)-\sum_{i=1}^{n} \ell_{i} \dot{\gamma}_{j}(t)
$$

This expression may be rewritten in terms of the neuromuscular time constant $\tau_{N}$,

$$
\tau_{N} \dot{u}_{p}(t)+u_{p}(t)=-e_{e}^{*} \gamma(t) ; \quad e_{e}^{*}=\left(e^{*}, 0\right)
$$

with $\tau_{N}=\frac{1}{\ell_{n+1}}$ and $\ell_{i}^{*}=\frac{\ell_{i}}{\ell_{n+1}} ; i=1,2, \ldots . n$. In the frequency domain, the existence of the first order lag is evident from observing 


$$
u_{p}(s)=\frac{-1}{\tau_{N} s+1} e_{e}^{*} \bar{\gamma}(s)
$$

To reflect the pilot's imprecise control input, a Gaussian white noise $v_{m}$ (refer to Figure 3.1 ) with covariance $v_{m}$ is added to the commanded input, $u_{c}$,

$$
\tau_{N} \dot{u}_{p}(t)+u_{p}(t)=u_{c}(t)+v_{m}(t)
$$

where $u_{c}(t)=-e_{e}^{*} \bar{\gamma}(t)$. This expression assumes that the control law and gains remain unchanged in the presence of this noise, and represents now a sub-optimal controlier.

Fortunately, the addition of motor noise changes the basic controlier structure very little from the previous development with only the augmented state vector $\bar{x}$ replacing $\bar{x}$, and $u_{c}$ replacing the deterministic $\bar{u}$. Incorporating Eq. (3.56) with Eq. (3.64), the modified state space representation follows as

$$
\begin{aligned}
& \dot{\bar{x}}(t)=A_{1} \bar{x}(t)+b_{1} u_{c}(t)+\bar{w}_{1}(t) \\
& \bar{y}_{p}(t)=c_{1} x(t-\tau)+\bar{v}(t-\tau) ; \bar{v} \sim N(0, v)
\end{aligned}
$$

with

$$
A_{1}=\left[\begin{array}{c|c}
A & b \\
\hline 0 & -\frac{1}{\tau_{N}}
\end{array}\right] \quad b_{1}=\left[\begin{array}{c}
0 \\
- \\
\frac{1}{\tau_{N}}
\end{array}\right]
$$

and $\quad C_{1}=\left[\begin{array}{lll}C & 0 & 0\end{array}\right],\left(\right.$ same as $\left.C_{0}\right)$.

The augmented noise vector $\bar{w}_{1}^{\prime}=\left(\bar{w}^{\prime}(t), \frac{v_{m}(t)}{{ }^{\tau} N}\right)$ has a covariance matrix of the form 


$$
\begin{aligned}
& E\left\{\bar{w}_{1}(t) \bar{w}_{1}^{\prime}(\sigma)\right\}=w_{1} \delta(t-\sigma) \\
& w_{1}=\left[\begin{array}{c|c}
w & 0 \\
\hline 0 & \frac{V_{m}}{\tau} N^{2}
\end{array}\right]
\end{aligned}
$$

Thus, based on the pilot's preceived observation, $\bar{y}_{p}$, the Kalman Filter generates the best estimate of $\bar{x}(t-\tau)$, or $\hat{\bar{x}}(t-\tau)$, from

$$
\begin{aligned}
& \dot{\hat{\bar{x}}}(t-\tau)=A_{1} \hat{\bar{x}}(t-\tau)+b_{1} u_{c}(t-\tau) \\
& +\bar{\Sigma}_{1} C_{1}^{\prime} v^{-1}\left[y_{p}(t)-C_{1} \hat{\bar{x}}(t-\tau)\right]
\end{aligned}
$$

with the error covariance, $\bar{\Sigma}_{\eta}$, satisfying

$$
0=A_{1} \bar{\Sigma}_{1}+\bar{\Sigma}_{1} A^{\prime}+W_{1}-\bar{\Sigma}_{1} C_{1}^{\prime} V^{-1} C_{1} \bar{\Sigma}
$$

As before, the predictor generates $\bar{\gamma}$, the least-mean-squared estimate of $\hat{\bar{x}}(t)$ based upon the delayed estimate of $\bar{x}(t-\tau)$, or $\hat{\bar{x}}(t-\tau)$ from

$$
\begin{aligned}
& \dot{\gamma}(t)=\bar{\xi}(t)+e^{A_{1} \tau}[\hat{\bar{x}}(t-\tau)-\bar{\xi}(t-\tau)] \\
& \dot{\bar{\xi}}(t)=A_{1} \xi(t)+b_{1} u_{c}(t)
\end{aligned}
$$

And, using techniques of Appendix $B$, state covariance ${ }^{[2]}$ of $\bar{\chi}$ becomes

$$
\begin{aligned}
X= & E\left\{\bar{x}(t) \bar{x}^{\prime}(t)\right\}=e^{A_{1} \tau} \bar{\Sigma}_{1} e^{A_{j}^{\prime} \tau}+\int_{0}^{\tau} e^{A_{1} \xi} W_{1} e^{A_{j}^{\prime} \xi} d \xi \\
& +\int_{0}^{\infty}\left(e^{\bar{A}_{\sigma} \sigma} e^{A_{1} \tau} \bar{\Sigma}_{1} C_{1}^{\prime} V^{-1} C_{1} \bar{\Sigma}_{1} e^{A_{1}^{\prime} \tau} e^{\bar{A}^{\prime} \sigma}\right) d \sigma
\end{aligned}
$$

with $\bar{A}=A_{0}-b_{0} l$. Thus,

$$
E\left\{x_{i}^{2}(t)\right\}=x_{i j} \quad i=1,2, \ldots n
$$




$$
\begin{aligned}
& E\left\{y_{i}^{2}(t)\right\}=\left(c_{1} \times c_{1}^{1}\right)_{j i} \quad i=1,2, \ldots m \\
& E\left\{u_{p}^{2}(t)\right\}=x_{n+1}, n+1
\end{aligned}
$$

To finally complete the development of the pilot model, two additional human limitations, observation thresholds and scanning behavior, must be included. Observation thresholds and shared attention a170cation increase the effective observation noise, $\bar{v}$. As stated before, the observation error $\bar{v}(t)$ is a zero mean Gaussian white noise process with covariance $v$. This covariance or $v_{j},{ }^{[5]}$ of the ith observed variable is modified to be

$$
v_{i}=\left[\frac{\rho_{i}}{f_{j} N\left(\sigma_{i}, \alpha_{i}\right)^{2}} \sigma_{i}^{2}\right]
$$

with $\sigma_{i}{ }^{2}$ the variance of the observed variable and $\rho_{i}$, the basic full. attention noise-to-signal ratio for human perception of this variable. Scanning behavior is accounted for by including in the above relation the parameter $f_{i}$, the fraction of total attention alloted to the ith observed variable. And, the perceptual or indifference threshold level, $\alpha_{i}$, influences the measurement noise covariance in the form of a dead-zone describing function, $N\left(\sigma_{i}, \alpha_{i}\right)$, with the following limits;

$$
\begin{aligned}
&\left|N\left(\sigma_{i}, \alpha_{i}\right)\right|=1 ;\left(\alpha_{i}=0,\right. \text { minimum variance of ith observed } \\
&\text { variable, } \left.v_{i}=\left(\frac{\rho_{i}}{f_{i}}\right) \sigma_{i}{ }^{2}\right) \\
&\left|N\left(\sigma_{i}, \alpha_{i}\right)\right|=0 ;\left(\sigma_{i}<\alpha_{i},\right. \text { no preception of ith variable, } \\
&\left.v_{i} \rightarrow \infty\right)
\end{aligned}
$$


When the signal's standard deviation, $\sigma_{j}$, is greater than the corresponding threshold level, the noise-to-signal ratio is increased by the magnitude of $\frac{1}{T N\left(\sigma_{i}, \alpha_{i}\right) T^{2}}$.

Thus, with the structure of the optimal control model established, Chapter 3 concludes with a section devoted to finding the pilot's frequency response from the mode?.

\subsection{Pilot's Frequency Response}

Given the pilot's objective, his control actions are assumed to be governed by the linear, time invariant equations of the optimal controller presented in the previous section. The objective of this section is to represent the optimal control model in the frequency domain, obtaining a transfer function matrix, $\bar{H}$, relating the pilot's observations $\bar{y}$ to his control output $u_{p}$, or

$$
u_{p}(s)=\bar{H}(s) \bar{y}(s)
$$

Taking the Laplace transform of Eq. (3.69) yields

$$
\bar{\gamma}(s)=\bar{\xi}(s)+e^{A_{1} \tau}\left[e^{-I s \tau}(\hat{\bar{\chi}}(s)-\bar{\xi}(s))\right]
$$

Collecting similar terms

$$
\begin{aligned}
e^{\left(I s-A_{1}\right) \tau} \bar{\gamma}(s)=\left[e^{\left(I s-A_{1}\right) \tau}\right. & -I] \bar{\xi}(s) \\
& +\hat{\bar{\chi}}(s)
\end{aligned}
$$

Next, transforming the equation that generates $\bar{\xi}(t)$, Eqn. (3.69), yields

$$
\bar{\xi}(s)=-\left(s I-A_{1}\right)^{-1} b_{q} e_{e}{ }^{*} \bar{\gamma}(s)
$$

Letting

$$
\hat{A}=A_{1}-\bar{\Sigma}_{1} C_{1}^{1} V^{-1} C_{1}
$$


the state estimator, from Eq. (3.67), is transformed to

$$
(I s-\hat{A}) \hat{\bar{x}}(s)+b_{1} e_{e}^{{ }^{*}} \bar{\gamma}(s)=\bar{\Sigma}_{1} C_{1}^{\prime} V^{-1} \bar{y}(s)
$$

and where $e^{-\tau S}$ has been factored from both sides substituting Eq. into Eq. (3.78) yields

$$
\begin{aligned}
\hat{\tilde{x}}(s)= & \left\{e^{\left(s I-A_{1}\right) \tau}+\left[e^{\left(s I-A_{1}\right) \tau}-I\right]\right. \\
& \left.\cdot\left(s I-A_{1}\right)^{-1} b_{l} e_{e}^{*}\right\} \bar{\gamma}(s)
\end{aligned}
$$

the transfer matrix between the estimated state and the predicted state,

$$
\begin{aligned}
\frac{\dot{\bar{\gamma}}(s)}{\hat{\bar{\chi}}(s)} & =\left\{e^{\left(s I-A_{1}\right)}+\left[e^{\left(s I-A_{1}\right) \tau}-I\right]\right. \\
& \cdot\left(s I-A_{7}\right)^{-1} b_{7} e^{*} e^{*-1}
\end{aligned}
$$

Substituting Eq. (3.80) into Eq. (3.81), creates the transfer matrix between the observation vector and the preaicted state

$$
\begin{aligned}
\frac{\bar{y}(s)}{\bar{y}(s)}=\left[( I S - \hat { A } ) \left\{e^{\left(S I-A_{1}\right) \tau}+\left[e^{\left(s I-A_{1}\right) \tau}-I\right]\right.\right. \\
\left.\left.\cdot\left(s I-A_{1}\right)^{-1} b_{1} e_{e}^{*}\right\}+b_{1} e_{e}^{*}\right]^{-1} \bar{\Sigma}_{7} c_{1}^{\prime} v^{-1}
\end{aligned}
$$

This expression can be rewritten as

$$
\begin{aligned}
\frac{\bar{y}(s)}{\bar{y}(s)} & =\left[( s I - \hat { A } ) \left\{e^{\left(s I-A_{1}\right)_{\tau}}-I\right.\right. \\
& \left.+\left[e^{\left(s I-A_{1}\right)_{\tau}}-I\right]\left(s I-A_{1}\right)^{-1} b_{1} e_{e}^{*}\right\} \\
& +(s I-\hat{A})+b_{1} e_{e}^{*} e^{-1} \bar{\Sigma}_{1} c_{1}^{1} v^{-1}
\end{aligned}
$$


or

$$
\begin{aligned}
& =\left[( s I - \hat { A } ) \left[\left[e^{\left(s I-A_{1}\right)_{\tau}}-I\right]\left(s I-A_{\eta}\right)^{-1}\left(s I-A_{\eta}\right)\right.\right. \\
& \left.+\left[e^{\left(s I-A_{1}\right)_{\tau}}-I\right]\left(s I-A_{\eta}\right)^{-1} b_{\eta} e_{e}^{*}\right\} \\
& +s I-\hat{A}+b_{q} e_{e}^{*} e^{-1} \dot{\Sigma}_{q} C_{\eta}^{1} V^{-1}
\end{aligned}
$$

or

$$
\begin{aligned}
& =\left[( s I - \hat { A } ) [ e ^ { ( s I - A _ { 1 } ) _ { T } } - I ] ( s I - A _ { 1 } ) ^ { - 1 } \left\{s I-A_{1}\right.\right. \\
& \left.\left.+b_{q} e_{e}^{*}\right\}+s I-\hat{A}+b_{q} e_{e}^{*}\right]^{-1} \bar{\Sigma}_{q} C_{\eta}^{1} V^{-1} .
\end{aligned}
$$

Setting

$$
\begin{aligned}
& \bar{A}=A_{1}-b_{q} e_{e}^{*} \text { and noting } \\
& {\left[e^{\left(s I-A_{1}\right) \tau}-I\right]\left(s I-A_{1}\right)^{-1}=\int_{0}^{\tau} e^{\left(s I-A_{1}\right) \sigma} d \sigma}
\end{aligned}
$$

establishes the general form of the transfer matrix between the observation vector and the predicted state, $\bar{\gamma}(t)$.

$$
\begin{aligned}
\frac{\bar{\gamma}(s)}{\bar{y}(s)} & =\left[(s I-\hat{A}) \int_{0}^{\tau} e^{\left(s I-A_{1}\right) \sigma} d \sigma(s I-\bar{A})\right. \\
& +s I-\hat{A}+b_{\eta} e_{e^{*}}^{-1} \bar{\Sigma}_{\eta} c_{\eta} V^{-1}
\end{aligned}
$$

The pilot transfer function $\bar{H}(s)$ considers $y_{m}$ to be zero, consistent with the definition of a transfer function. Thus

$$
\tau_{N} \dot{u}_{p}(t)+u_{p}(t)=-e_{e}^{*} \bar{\gamma}(t)
$$

is transformed to 


$$
\frac{u_{p}(s)}{\bar{y}(s)}=\frac{-\ell_{\mathrm{e}}^{*}}{\tau_{N} s+1}
$$

Multiplying Eq. (3.90) with Eq. (3.88) produces the desired pilot transfer function

$$
\begin{aligned}
\frac{u_{p}(s)}{\bar{y}(s)} & =\frac{-e_{e}^{*}}{\tau_{N} s+T}\left[(s I-\hat{A}) \int_{0}^{\tau} e^{\left(s I-A_{1}\right) \sigma} d \sigma(s I-\bar{A})\right. \\
& \left.+s I-\hat{A}+b_{1} e_{e}^{*}\right]^{-1} \bar{\Sigma}_{q} C_{1} V^{-1}
\end{aligned}
$$

Other forms of $\bar{H}(s)$ are also possible. Now, the pilot's frequency response may then be obtained simply by substituting $s=j w$ in the expression, above. 


\section{CHAPTER 4}

\section{METHODOLOGY AND RESULTS}

Thus far, a decade of dust has been blown off two interesting developments. The first, reca11, was a hypothesis correlating pilot opinion with elements of pilot compensation and closed-loop performance. Although early results verifying this hypothesis were encouraging, problems inherent to the classical model structure plagued the approach. Contrary to this situation, the OCM was frequentiy applied to many situations involving the human controller. In fact, the OCM was originally viewed as a viable alternative to the classical model structure, minimizing many of the shortcomings of the classical method.

Thus as the next step, in Chapter 4 , we must first merge the $0 \mathrm{CM}$ modelling procedure with the hypothesis and methodology of Neal and Smith to produce the desired alternate approach. Secondly, in Chapter 4, we must utilize the new approach to develop the results for a number of configurations originally analyzed by Neal and Smith, and then compare these results.

To logically interpret the Neal-Smith procedure via the OCM, Chapter 4 is divided into four sections, with the first containing a comparison of the two interpretations of tracking strategy. The second section, then, builds on the discussion of the first, providing a model of the tracking task. This second section also encompasses the identification of the $\mathrm{OCM}^{\prime} \mathrm{s}$ parameters, vectors, and matrices 
relevant to defining the task. The third section exercises the optimal solution of the problem as stated in Section 4.2 in that it uses the resulting pilot frequency response (defined as Eq. (3.91)) to duplicate the Neal and Smith methodology. Lastly, the fourth section presents the results and the proposed design criteria of the new approach, providing in addition an interesting correlation between bandwidth and pilot rating.

But first, consider the OCM's tracking strategy in comparison to that interpreted by Neal and Smith.

\subsection{Basic Hypothes is}

Surely, good tracking minimizes the error between the target's attitude and the aircraft's attitude. The addition of tracking error $\left(\theta_{c}-\theta\right)$ to $J_{p}$ results in an OCM control strategy that minimizes the mean squared value of error, which can be expressed as

$$
\sigma_{E}^{2}=\frac{1}{\pi} \int_{0}^{\infty}\left|1-\frac{\theta}{\theta_{c}}(j \omega)\right|^{2} S_{\theta_{c}}(\omega) d \omega
$$

where $S_{C}$ is the spectral density of the commanded signal. Minimizing the above expression may be interpreted as forcing the closed-loop frequency response $\left(\frac{\theta}{\theta_{c}}\right)$ to tend to unity over the frequency range of the commanded signa1. Unity corresponds to the performance attained by the perfect tracker with $\left|\frac{\theta}{\theta_{c}}\right|=0 \mathrm{~dB}$ and $\dot{ }\left(\frac{\theta}{\theta_{c}}\right)=0^{\circ}$. Thus, in terms of amplitude of the closed-loop frequency response, the OCM "automatically" minimizes droop and resonance peak.

In fact, the OCM goes beyond Neal and Smith's definition of a good tracker to create a more correct definition, incorporating the effects of the closed-10op phase $\Varangle\left(\frac{\theta}{\theta_{c}}\right)$ characteristics on low frequency 
$(\omega \leq B \omega)$ performance. The early analysis was only interested in one aspect of closed-loop phase, and that was bandwidth. Unfortunately, two systems with the same bandwidth and droop characteristics will sometimes exhibit markedly different levels of low frequency tracking performance.

Truiy, the pilot is just as interested in minimizing the distance between closed-1oop phase $\$\left(\frac{\theta}{\theta_{c}}\right)$ and the zero degree datem (or the phase difference between command and output), as he is in minimizing droop (or differences in the magnitudes). Having one objective satisfied without the other could result in systems exhibiting excessive lag, even in cases with acceptable bandwidth. Unlike Neal and Smith's pilot modeling concept, the $\mathrm{OCM}$ is concerned with both the low-frequency amplitude and the low-frequency phase of $\left(\frac{\theta}{\theta_{C}}\right)$ in minimizing tracking error. And as a result of the optimization algorithm, the OCM will "automatically" determine the bandwidth required to achieve the pilot's objective within the abilities of the vehicle dynamics.

In summary, Neal and Smith proposed that the pilot is trying to achieve good low frequency performance (a reasonable bandwidth with a minimum of low frequency droop) plus good high frequency stability $\left(\left.|\theta| \theta_{c}\right|_{\max }\right.$ as small as possible). This proposition is a frequency domain representation of the pilot's objective to minimize tracking error. In contrast, the OCM provides another frequency domain representation of the pilot's strategy, encompassing the objectives of the previous approach in that it produces a tracker that attempts to be ideal across the frequency range of the commanded signa?. 


\subsection{Modeling the Task}

To produce the desired tracker, the OCM must include a model of the tracking task. Three key elements concerning the application of the pilot model must be established:

(1) The pilot's observations and objective function to be minimized.

(2) The system's representation in a tracking task.

(3) A definition of the command signal to be tracked.

In this discussion, specific attention will be given to adapting the OCM transfer functions to the simplified compensatory tracking task model of Neal and Smith. But first, the appropriate objective function must be selected.

The optimal control model's control input is selected to minimize the following objective function

$$
J_{p}=E\left\{\lim _{T \rightarrow \infty} \frac{1}{T} \int_{0}^{T}\left(\bar{y}^{\prime} Q \bar{y}+r u_{p}^{2}+g \dot{u}_{p}^{2}\right) d t\right\}
$$

subject to human limitations. The weighting matrix, $Q_{y}=\operatorname{diag}\left[q_{1}, q_{2}\right.$, $\left.\ldots, q_{m}\right] \geq 0^{\prime}\left(Q_{y} \doteq C^{\prime} Q C\right.$ from Chapter 3$)$ where $m$ is the dimension of the observation vector $\bar{y}$; the weightings on control and control rate, (scalars in this analysis) $r \geq 0$ and $g>0$; plus the elements of $\bar{y}$ must all be determined to quantify the task.

Obviously, the most critical parameter is the tracking error $\varepsilon$; the difference between the commanded attitude $\theta_{c}$ and the aircraft's attitude $\theta$. Observation of attitude itself is also required if the task is one of pursuit rather than compensatory in nature. (A compensatory task is defined such that only error is observed). In addition 
to $\varepsilon$ and $\theta$, studies $[2]$ have shown that the human controller can also extract rate as well as position from a single display, thereby expanding $\bar{y}$ to

$$
\bar{y}^{\prime}=(\varepsilon, \stackrel{\circ}{\varepsilon}, \theta, \dot{\theta})
$$

The next step in quantifying the pilot's control objective is selecting the cost function weightings. Fortunately, research has begun to shed some light on the critical task of weight selection. One study[6] attempted to identify the weighting matrix $Q$ by correlating pilot opinion ratings, the objective cost $J_{p}$, and performance statistics with simulation results. The investigation concluded that over a wide range of tracking tasks and flight conditions the following weights on $\varepsilon, \dot{\varepsilon}$ and $F_{\text {stick }}$

$$
q_{\varepsilon}=16, q_{\varepsilon}=1, r_{F_{s}}=0
$$

would accurately reflect the pilot's control objectives. The weighting, along with zero weighting on $\theta$ and $\dot{\theta}$ defines $Q$ and $r$ in the following analysis. It should be noted that this $Q$ emphasizes the pilot's primary goal of minimizing error with some constraint on how fast the error may fluctuate. To complete the definition of $\mathrm{J}_{p}$, the weighting on control rate must be set.

The weighting $g$ on the control rate $\dot{u}_{p}$ is constrained by physiological limits. These limits are linked with the neuromuscular dynamics associated with the human controller modeled as a first order lag. The associated lag time constant ${ }^{T_{N}}$ is expressed in the context of the pilot (model) control law, 


$$
\tau_{N} \dot{u}_{p}=-\ell_{e}^{*} \bar{\gamma}-u_{p}+v_{m}
$$

For a given set of weights on $\bar{y}$ and $u_{p}(r=0$ usually), adjusting $g$ in the cost function determines $\tau_{N}$. Finally, for aggressive control action, the lower limit on $\tau_{N}$ has been determined to be near 0.1 seconds, based on experimental, man-machine data.

The vehicle dynamics to be controlled must be represented by the linear time-invariant equations of motion:

$$
\dot{\bar{x}}(t)=A \bar{x}(t)+B u_{p}(t)+N(t)
$$

To model the tracking task, the vehicle states must be augmented with the command signal states. The augmented system is structured as follows:

$$
\begin{aligned}
& {\left[\begin{array}{l}
\bar{x}_{c} \\
\bar{x}
\end{array}\right]=\left[\begin{array}{l|l}
A_{c} & 0 \\
\hline 0 & A_{v e h}
\end{array}\right] \cdot\left[\begin{array}{l}
\bar{x}_{c} \\
\bar{x}
\end{array}\right]+\left[\begin{array}{l}
0 \\
- \\
b_{v e h}
\end{array}\right] u_{p}+\left[\begin{array}{l}
e_{c} \\
- \\
0
\end{array}\right] w} \\
& \bar{y}=\left[\begin{array}{c:cc}
I_{2} & -I_{2} \\
\hdashline 0 & I_{2}
\end{array}\right]-\left[\begin{array}{l}
\bar{x}_{c} \\
\bar{x}
\end{array}\right]+\bar{v}
\end{aligned}
$$

where the vehicle states are defined as $\bar{x}^{\prime}=[\theta, \dot{\theta}, \alpha$, etc. $]$, the command signal states $\bar{x}^{\prime}=\left[\theta_{c}, \dot{\theta}_{c}\right]$, and $I_{(\cdot)}$ indicates the identity matrix of appropriate dimension.

In this analysis the commanded attitude is generated by a second order filter driven with white noise, or

$$
\ddot{\theta}_{c}+.5 \dot{\theta}_{c}+.25 \theta_{c}=.25 w(t)
$$


Table 4.1 Baseline pilot model

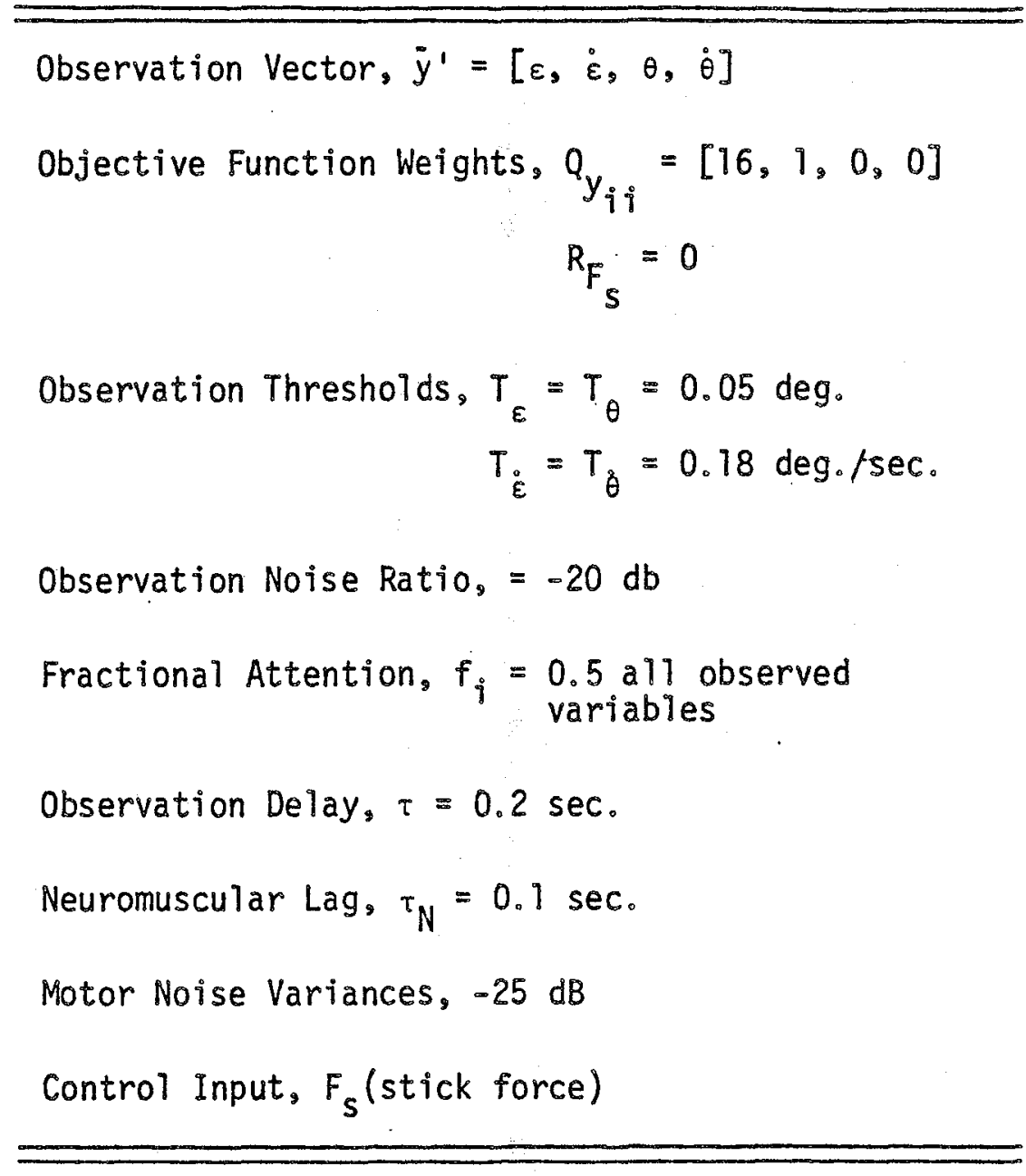

where $w \sim N(0,64)$. This commanded signal approximates the discrete tracking experiment performed in the Neal and Smith investigation. The statistics on $\theta_{c}$ and $\theta_{c}$ :

$$
\sigma_{\theta_{c}}^{2}=16 \operatorname{deg}^{2}, \quad \sigma_{\dot{\theta}_{c}}^{2}=4 \operatorname{deg}^{2} / \mathrm{sec}^{2}
$$

indicate a reasonable, yet sufficiently challenging task to test pitch attitude tracking. Defining the commanded signal completes the objectives of this section. Table [4.1] summarizes the resulting pilot mode 1 parameters. 


\subsection{Analysis Technique}

The discussion turns now to the acquisition of those parameters required for the analysis technique. Of paramount importance is the ability to obtain a frequency domain representation of a controller developed in the time domain.

Moreover, producing the system's closed-loop frequency response requires knowledge of both the pilot's transfer function matrix, $\bar{H}_{p}(j \omega)$, and the aircraft's transfer function, $H_{a}(j \omega)$. Using the following expression

$$
H_{a}(j \omega)=\frac{\theta(j \omega)}{u_{p}(j \omega)}=C\left(j \omega I-A_{v e h}\right)^{-1} b_{v e h}
$$

with $C=\left[\begin{array}{lllll}1 & 0 & 0 & \ldots & 0\end{array}\right]$, provides the plant's frequency response at selected values of $\omega$. And from Eq. (3.91), the pilot's frequency response $\bar{H}_{p}(j \omega)$ is obtained at each frequency.

To calculate the closed-loop frequency response, consider now the pilots control law resulting from the problem formulation of the previous chapter. By expanding the coherent part (ignoring motor noise) of pilot control in the frequency domain or

$$
\begin{aligned}
u_{p}(s) & =H_{\varepsilon}(s) \varepsilon(s)+H_{\varepsilon}(s) \dot{\varepsilon}(s) \\
+ & H_{\theta}(s) \theta(s)+H_{\dot{\theta}}(s) \dot{\theta}(s),
\end{aligned}
$$

the four dynamic components of $\bar{H}_{p}$ are revealed. The pilot's input may be rewritten as

$$
\begin{aligned}
u_{p}(s) & =\left[H_{\varepsilon}(s)+s H_{\varepsilon}(s)\right] \varepsilon(s) \\
& +\left[H_{\theta}(s)+s H_{\dot{\theta}}(s)\right] \theta(s)
\end{aligned}
$$


where the bracketed terms are the true transfer functions relating tracking error and attitude angle to pilot input, respectively. Defining the aircraft attitude transfer function as

and

$$
\theta(s)=H_{a}(s) u_{p}(s)
$$

$$
\varepsilon(s) \doteq \theta_{C}(s)-\theta(s)
$$

the desired closed loop transfer function is simply

$$
\frac{\theta(s)}{\theta_{c}(s)}=\frac{H_{2}(s)\left[H_{\varepsilon}+s H_{\varepsilon}\right]}{1+H_{a}(s)\left[\left(H_{\varepsilon}+s H_{\varepsilon}\right)-\left(H_{\theta}+s H_{\theta}\right)\right]}
$$

A block diagram of this $\frac{\theta}{\theta_{C}}$ transfer function is presented in Figure [4. 7].

The compensatory tracking model used by Neal and Smith, however, did not consider the inner loop $\left(\mathrm{H}_{\theta}+\mathrm{H}_{\theta} \mathrm{s}\right)$, even though the pilot monitored both error and attitude (i.e. pursuit task) in their experiment. To be consistent with the Neal-Smith model, make the following approximation; assume that the attitude's contribution to the pilot's compensation, after obtaining the complete model, is small. Or, the pilot's control input is approximately

$$
u_{p}(s) \cong\left(H_{\varepsilon}(s)+s H_{\varepsilon} \cdot(s)\right) \varepsilon(s)
$$

with $H_{\varepsilon}$ and $H_{\varepsilon}$ found with attitude observed by the pilot, as simulated. The resulting approximate closed-7oop transfer function is, of course

$$
\frac{\theta(s)}{\theta_{c}(s)}=\frac{H_{a}(s) H_{p}(s)}{1+H_{a}(s) H_{p}(s)}
$$

identical to the Neal and Smith compensatory tracking model. 

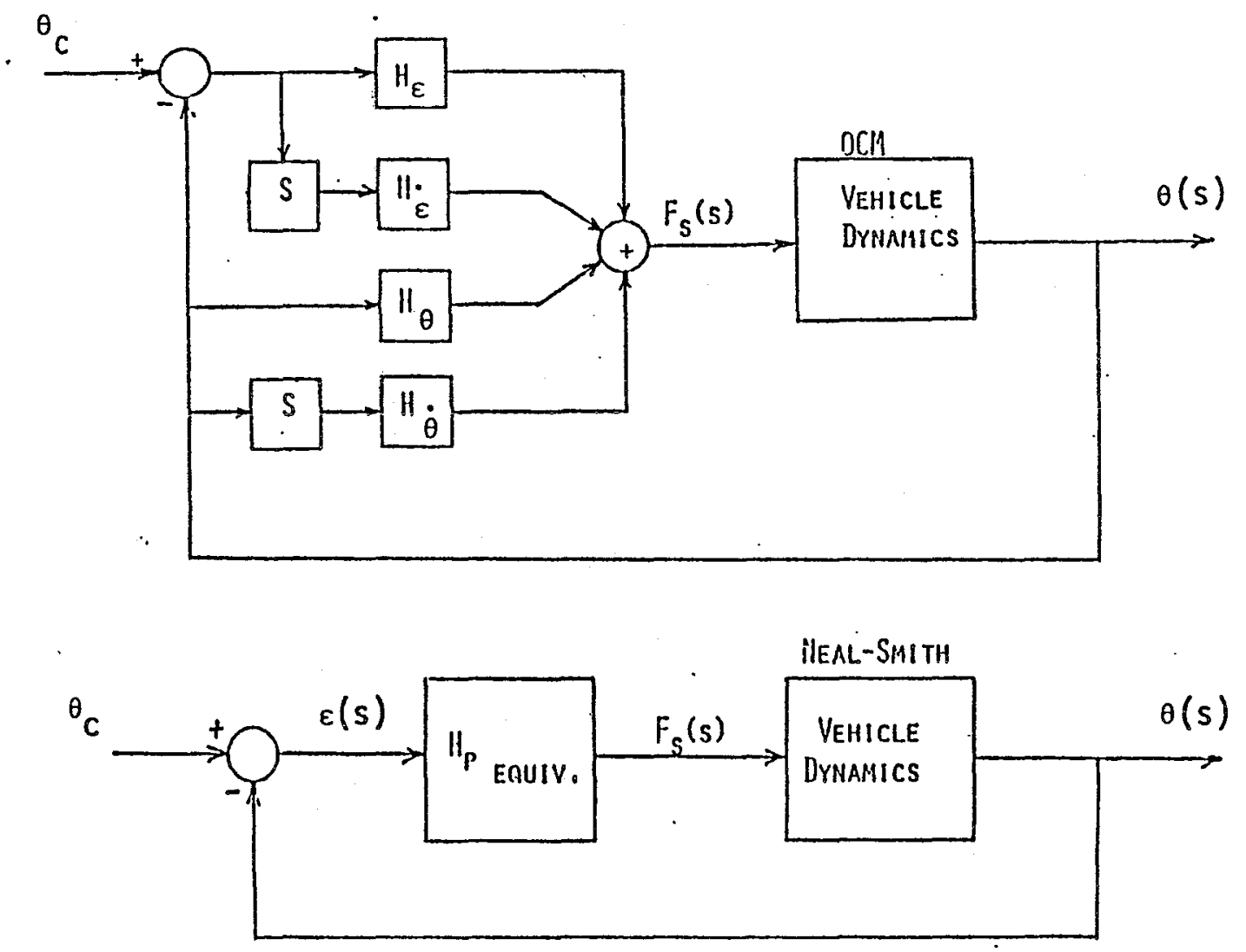

Figure 4.1 Model Schematic Comparison 
Additionally, one final difference should be noted. Unlike the Neal-Smith pilot strategy, the OCM's control strategy is dependent upon three additional stochastic inputs: the command process $\theta_{c}$, the motor noise process, and the observation noise process. Recall that due to the first, the pilot transfer vector $\bar{H}_{p}(s)$ produces a closedloop strategy that attempts to be ideal across the frequency range of $\theta_{c}$. Whereas the motor noise and observation noise, ignored by Neal and Smith by their not including any pilot remnant, affect the OCM by producing a control strategy designed to limit their effect. However, as will be shown, the effects of pilot remnant was negligible in obtaining meaningful closed-loop, steady-state results.

To relate frequency domain characteristics to time domain characteristics, one approach consists of constructing the spectral densities of the system's variables $\theta_{c}, \varepsilon_{g} u_{p}$ and $\theta_{0}$. The area under the spectral density $S_{x}$ is related to the respective variances $\sigma_{x}{ }^{2}$ by the relation

$$
\sigma_{x}^{2}=\frac{1}{\pi} \int_{0}^{\infty} S_{x}(\omega) d \omega
$$

if $x$ is a zero mean process. The perturbation variables $\theta_{c}, \varepsilon, u_{p}$ and $\theta$ are zero mean process, so integrating Eq. (4.17) will yield the system variances $\sigma_{\theta}^{2}, \sigma_{\varepsilon}^{2}, \sigma_{u_{p}}^{2}$, and $\sigma_{\theta}^{2}$. These variances may then be compared to their counterparts, obtained directly from the OCM's state covariance to see what effect neglecting pilot remnant and attitude feedback have on evaluating closed-loop performance.

One method of calculating the needed spectral densities, assuming $\theta_{c}$ as the only closed-loop system input (i.e. ignoring remnant), proceeds as follows: 


$$
\begin{aligned}
& S_{w}(\omega)=\sigma_{w}^{2} \\
& S_{\theta_{c}}(\omega)=\left|H_{q}(j \omega)\right|^{2} S_{w}(\omega) \\
& S_{\varepsilon}(\omega)=\left|\frac{\varepsilon}{\theta_{c}}(j \omega)\right|^{2} S_{\theta_{c}}(\omega) \\
& S_{u_{p}}(\omega)=\left|\frac{u_{p}}{\varepsilon}(j \omega)\right|^{2} S_{\varepsilon}(\omega) \\
& S_{\theta}(\omega)=\left|\frac{\theta}{u_{p}}(j \omega)\right|^{2} S_{u_{p}}(\omega)
\end{aligned}
$$

where $\sigma_{w}^{2}$ is the intensity of the white noise driving the $\theta_{c}$ process with $\mathrm{H}_{7}(\mathrm{~s})$ the shaping filter's transfer function. And in Eq. (4.20), the transfer function relating attitude error to command signal is obtained from

$$
\frac{\varepsilon}{\theta_{c}}(j \omega)=\frac{1}{1+H_{a}(j \omega) H_{p}(j \omega)}
$$

Once the spectral densities are evaluated over a sufficient band $(\omega)$, the integration can be performed graphically to obtain the desired variances. A sample of these variances and their OCM-derived counterparts are presented in Table [4.2] for configuration $2 \mathrm{~A}$.

Table 4.2 RMS Comparison for configuration $2 \mathrm{~A}$

\begin{tabular}{ccc} 
& Reduced System & Actual (OCM) \\
\hline$\sigma_{\theta_{C}}$ (deg) & 3.943 & 4.000 \\
$\sigma_{\varepsilon}$ (deg) & 0.824 & 0.808 \\
$\sigma_{F_{s}}$ (lbs) & 2.289 & 2.496 \\
$\sigma_{\theta}$ (deg) & 3.670 & 3.840 \\
\hline \hline
\end{tabular}


Surprisingly, the effect of cancelling the inner attitude loop and the motor and observation noises made little impact on the system's variances. Thus, the simpler closed-loop structure is a close approximation of the OCM's closed-loop structure. Conversely, the OCM's format is now a valid model for the Neal and Smith "pilot-in-the-loop analysis". It remains to identify. the procedure and the resulting parameters deemed critical to pilot/vehicle analysis.

The procedure begins by considering the output of the frequency analysis in a computer package for pilot-model analysis, or PIREP[5].

PIREP may be used to calculate the frequency domain characteristics (Bode plots) of both the open-loop OCM pilot, $H_{p}(j \omega)$, and the open-loop vehicle, $H_{a}\left(j_{\omega}\right)$, and these are determined in terms of amplitude (in $d B$ ) and phase (in deg) for a selected set of input frequencies. Thirty-one points, Tisted in Table 4.3 , along the frequency scale were judiciously chosen to provide an accurate representation of the frequency response an a logarithmic scale. The Bode-plot

Table 4.3 Selected Frequencies

\begin{tabular}{rlllll}
\hline no. & Frequency $^{*}$ & no. & Frequency $^{*}$ & no. & Frequency $^{*}$ \\
\hline 1 & 0.060 & 11 & 2.813 & 29 & 6.596 \\
2 & 0.130 & 12 & 3.063 & 22 & 7.183 \\
3 & 0.250 & 13 & 3.335 & 23 & 7.822 \\
4 & 0.500 & 14 & 3.632 & 24 & 8.518 \\
5 & 1.000 & 15 & 3.955 & 25 & 9.276 \\
6 & 1.500 & 16 & 4.307 & 26 & 10.100 \\
7 & 2.000 & 17 & 4.690 & 27 & 11.000 \\
8 & 2.178 & 18 & 5.108 & 28 & 16.000 \\
9 & 2.372 & 19 & 5.562 & 29 & 22.000 \\
10 & 2.583 & 20 & 6.057 & 30 & 32.000 \\
& & & & 31 & 40.0 \\
& & & & $*($ in rad/sec) \\
\hline
\end{tabular}


format of $H_{a}(j \omega)$ and $H_{p}(j \omega)$, corresponding to each w listed, al lowed easy calculation of the open-loop pilot/vehicle system

$$
\begin{aligned}
& \left|\frac{\theta}{\theta_{e}}(j \omega)\right|_{\text {in } d B}=\left|H_{a}(j \omega)\right|_{\text {in } d B}+\left|H_{p}(j \omega)\right|_{\text {in } d B} \\
& \forall\left\langle\frac{\theta}{\theta_{e}}(j \omega)\right)=ł\left\langle H_{a}(j \omega)\right)+\Varangle\left\langle H_{p}(j \omega)\right) .
\end{aligned}
$$

Unfortunately, to find the closed-loop frequency response more care must be given in selecting the frequencies $\omega$, since important details (i.e. $\left|\frac{\theta}{\theta_{e}}\right|$ max ) can be hidden between sample points. Clearly avoiding the uneconomical solution of the $\mathrm{OCM}$ generating more points, this analysis relied on a cubic-spline interpolation of the two smooth open-loop $\left(\frac{\theta}{\theta_{e}}(j \omega)\right)$ curves to generate the additional closed-loop points.

Once the interpolation procedure has boosted the total number of open-10op points to 200 , these points were then translated to their complex number equivalents. The closed-1oop frequency response is obtained simply by

$$
\frac{\theta}{\theta_{c}}(j \omega)=\frac{\frac{\theta}{\theta_{e}}(j \omega)}{1+\frac{\theta}{\theta_{e}}(j \omega)}
$$

for each interpolated value of $\frac{\theta}{\theta_{e}}$. This expression produces a sequence of 200 complex numbers to be translated into closed-loop Bode plots, or amplitude and phase characteristics. In this way, bandwidth, droop and resonant peak can be accurately measured and displayed. Furthermore, the same cubic-spline interpolation scheme is applied to $H_{p}(j \omega)$ to obtain information regarding pilot compensation. As an example, 
consider Figures (4.2 and 4.3), displaying results obtained from the analysis of configuration $2 \mathrm{D}$.

Bandwidth, recall, is defined as the frequency at which the closedloop phase $k\left(\theta / \theta_{c}\right)$ is $-90^{\circ}$. Unlike the Neal and Smith study, bandwidth is now a variable, dependent on task, vehicle, and human factors. Clearly, neuromuscular $\operatorname{lag} \tau_{N}$ effects all four elements of the pilot transfer vector $\bar{H}_{p}(s): H_{\varepsilon}(s), H_{\varepsilon}(s), H_{\theta}(s)$, and $H_{\dot{\theta}}(s)$. Aithough $\tau_{N}{ }^{\prime} s$ effect on the pilot's characteristics is self-evident, it is the notso-self-evident effect on closed-loop bandwidth (speed of response) that is of interest. As $\tau_{N}$ increases, the closed-loop bandwidth decreases. Therefore, "relaxed" pilot behavior, or large $\tau_{N}$, is associated with a closed-loop system exhibiting slow response characteristics. Conversely, aggressive pilot behavior, or a low $\tau_{N}$, produces a higher bandwidth, producing a more responsive and more aggressive pilot/aircraft combination. Since, then, the minimum $\tau_{N}$ is usually accepted to be $0.1 \mathrm{sec}$ for aggressive tracking, setting this value in the OCM determines the maximum achievable bandwidth for the system.

The second measure is the pilot phase compensation. The total pilot phase compensation from the OCM is the phase angle of the pilot's frequency response evaluated at the system bandwidth frequency $(\omega=B w)$. This compensation, however, includes the effects of neuromuscular $\operatorname{lag} \tau_{N}$ and the perceptual time delay $\tau$. These effects may be corrected for via the following expression

$$
\ddagger_{p c}=\Varangle_{O C M}{ }_{\omega=B W}+57.3 \tau \cdot B w+\tan ^{-1}\left(\tau_{N} \cdot B W\right)
$$


CONF IGURATION 2D
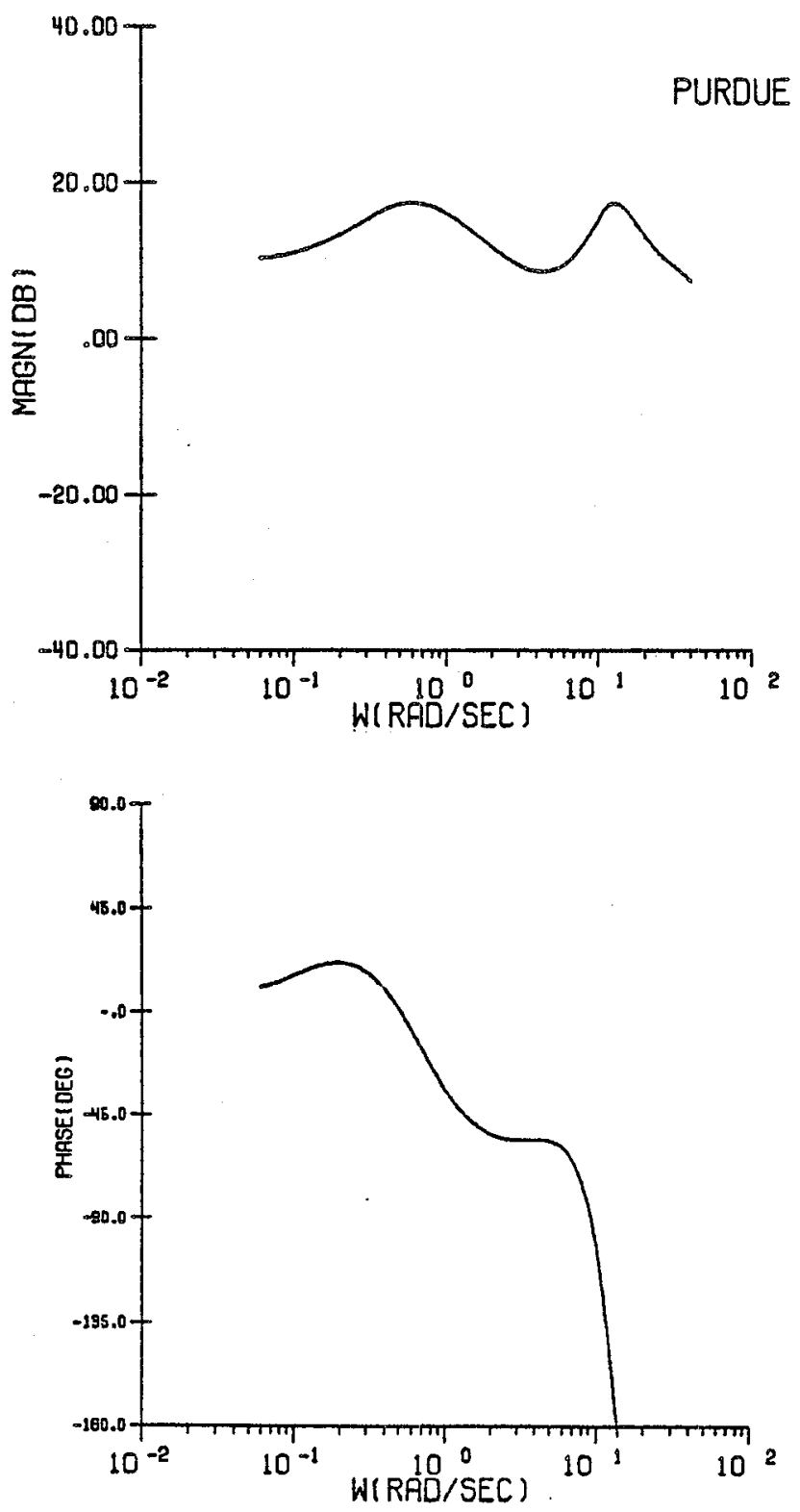

Figure 4.2 Pilot Frequency Response 
CONF IGURATION 20

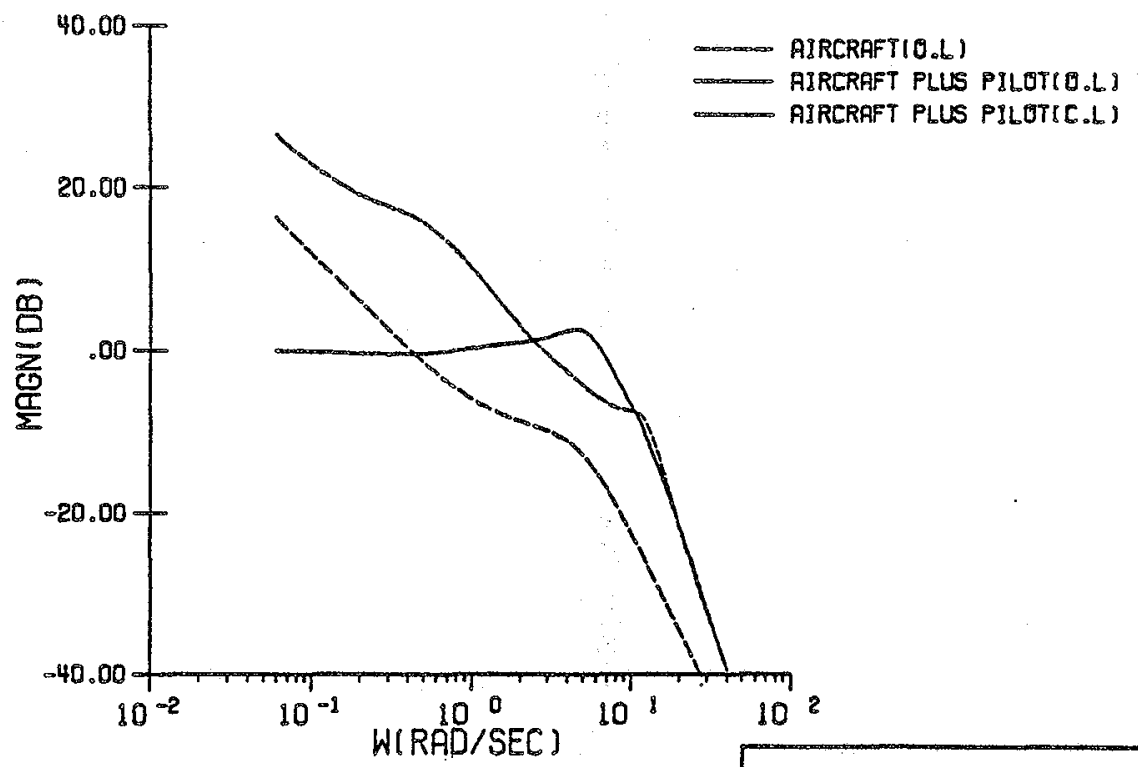

BANDWIOTHE 3.68 RAD/SEC PILOT COMPENSATION= -55.61 DEG

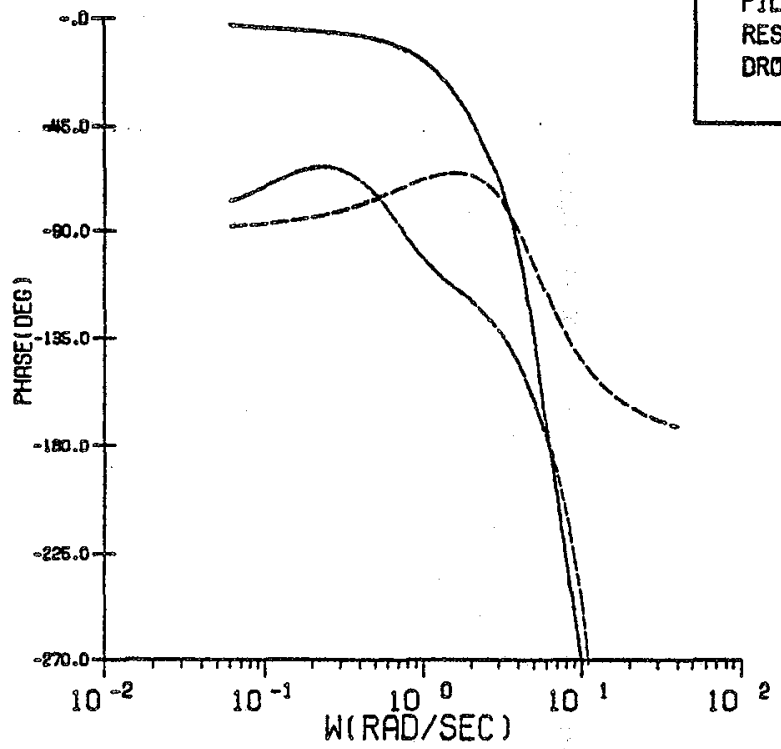

Figure 4.3 System Frequency Response 
where $\xi_{p c}$ corresponds to the Neal and Smith's interpretation of pilot compensation. For the purpose of correlation, Neal and Smith could have included their (constant) effective time delay as part of the pilot compensation. And since their bandwidth was fixed, this would simply slide the phase compensation scale (see Fig. 2.11) lower by a fixed angle for all aircraft configurations.

The last measure, magnitude of resonance peak, $\left|\frac{\theta}{\theta_{c}}\right|$ max is available from the closed-loop Bode plot, and the next section will explore it further. Hence all quantities are available through the OCM modeling process presented previously.

\subsection{Results from the Methodology}

Initial application of the method, discussed thus far, reveals the following characteristics of the $\mathrm{OCM}$ in evaluating the tracking task.

1) The closed-loop system bandwidth varies with vehicle, task, and human factors.

2) The (frequency response) droop varies with vehicle, task, and human factors.

3) Given a stabilizable and detectable system where $\left(A, \sqrt{C^{\prime} Q C}\right)$ is observable, the OCM will always produce a stable solution.

4) Without correction, the resonance peak's magnitude of the OCM could not be correlated with PIO tendencies.

At first glance, item number four would appear to be disastrous, especially since Neal and Smith correlated magnitude of resonance peak $\left|\frac{\theta}{\theta_{c}}\right|_{\max }$ with oscillatory tendencies. But given statement (3), the absence of a significant peak is not totally unexpected. For 
instance, given a configuration that is considered PIO prone (IG), the OCM will still produce a stable solution. A PIO condition will not be observed in the OCM's frequency response, since PIO tendencies, are by definition, unstable closed-loop solutions.

The severe PIO prone condition, while not being predominant in $\left|\frac{\theta}{\theta_{c}}\right|$ max , can be observed in the bandwidth frequency the pilot must settle for to obtain a stable system. Consider the definition given with PIO rating no. 4 :

Oscillations tend to develop when pilot initiates abrupt manuevers or attempts tight control. Pilot must reduce gain or abandon task to recover.

Typically, the pilot must "ease off," or fly the aircraft less aggressively, to avoid hazardous oscillatory tendencies.

Application of the method to several aircraft configurations evaluated by Neal and Smith resulted in bandwidth/pilot rating results shown in Figure [4.4]. These results reveal a trend of degraded pilot opinion with decreased closed-loop bandwidth, particularly where $\mathrm{Bw} \leq 3 \mathrm{rad} / \mathrm{sec}$ in this task. Pilot comments mention an inability to track without severe PIO problems in configurations $1 G, 1 F, 2 I$, and $2 G$, in support of the above results. Pilots also complained that these aircraft were "real sleepers", that is, they had large initial response delays, another indication of insufficient bandwidth.

The question of how to expose poor handiing when a sufficient bandwidth is present is answered by the results shown in Figure [4.5], in which pilot rating is shown to depend on closed-loop resonance and pilot's phase compensation. But obtaining these results requires 


\section{RATING/BANDHIDTH \\ CORRELATION}

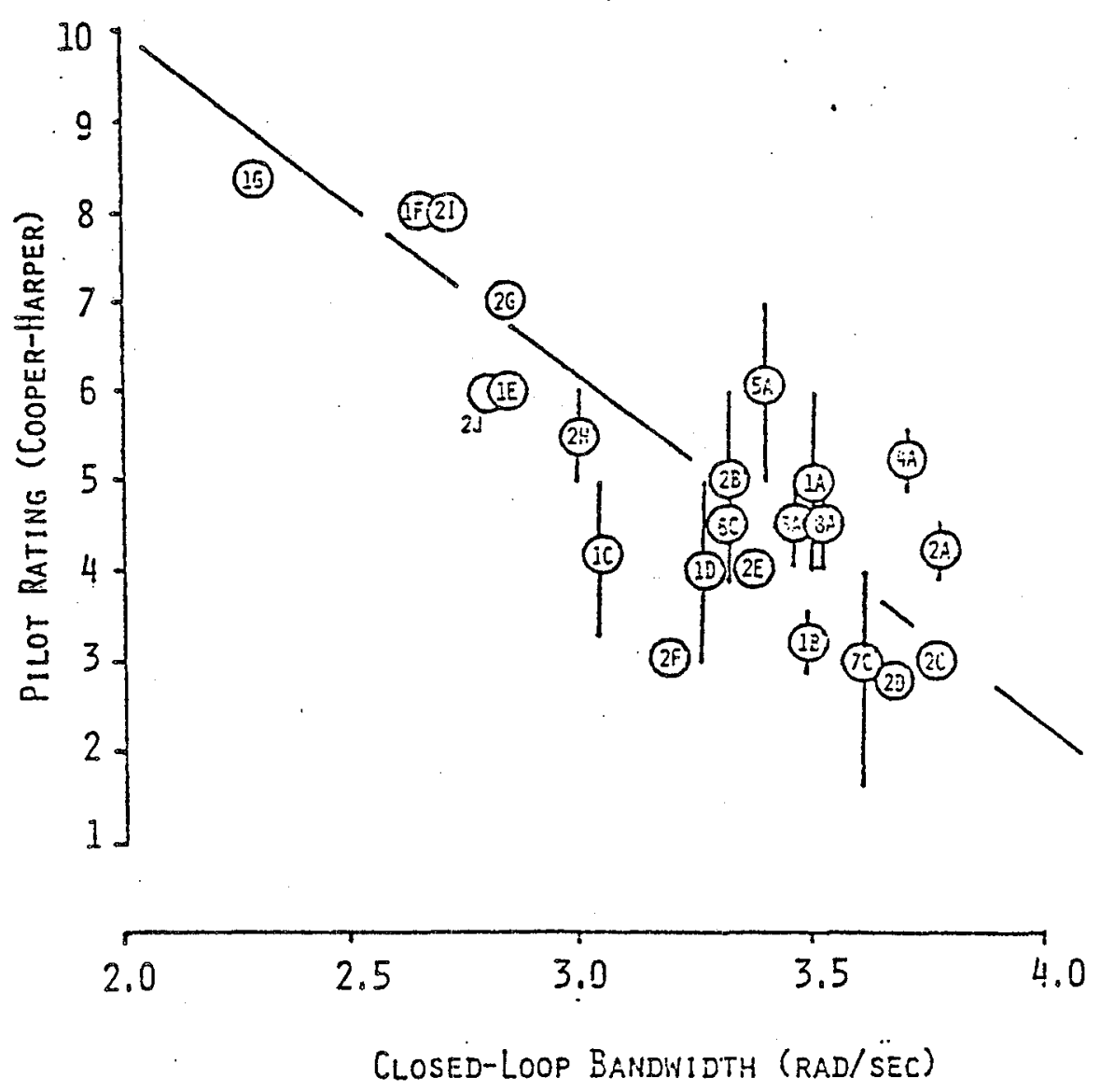

Figure 4.4 Pilot Rating/Bandwidth Correlation 


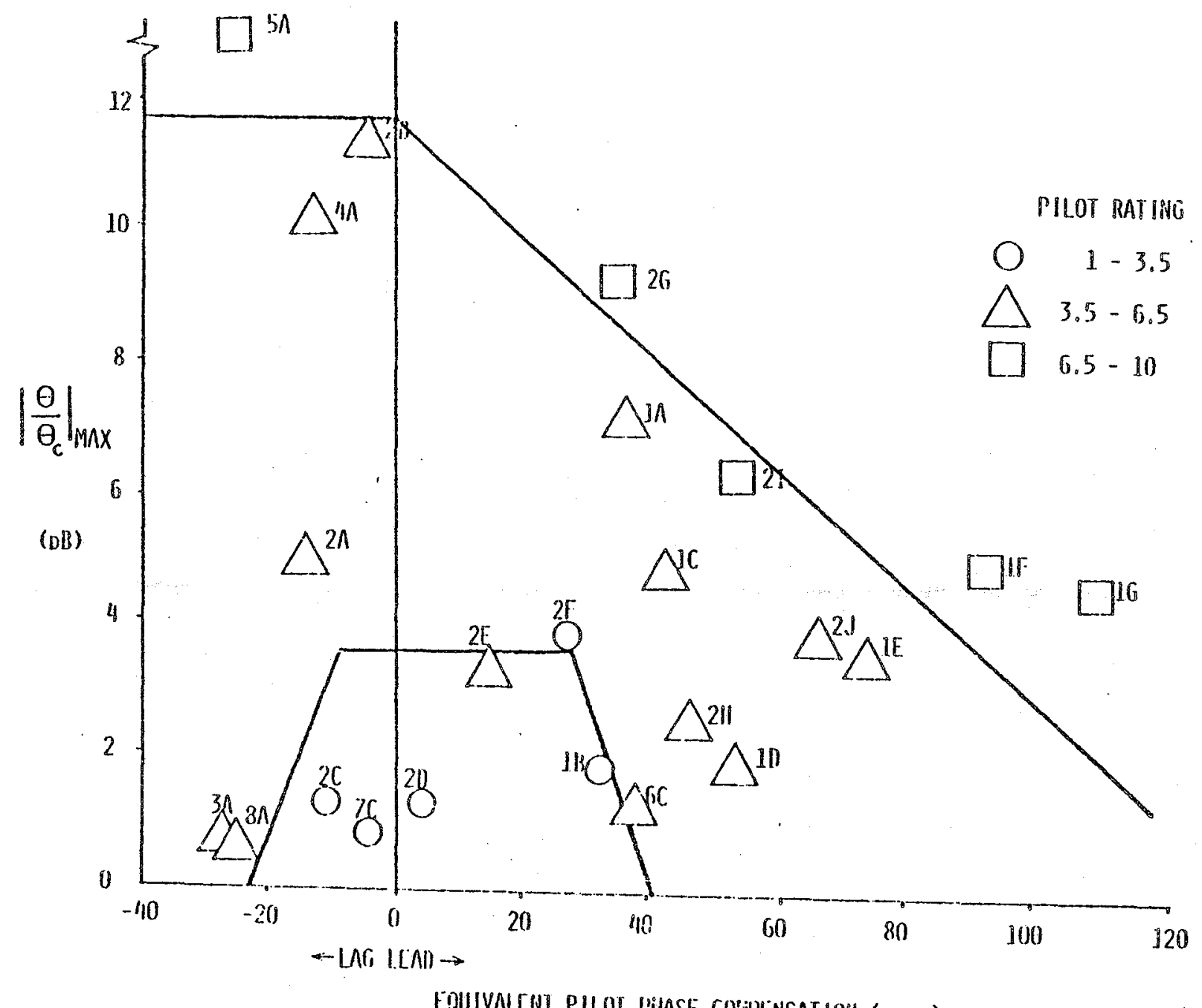

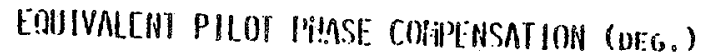

Figure 4.5 Results of Optimal Control Analysis 
discussion. With sufficient closed-loop bandwidth, large time delays of the initial responses are no longer present. The problem, which incidentally is evident in the rms tracking errors, is rooted in the trade off between errors due to low-frequency versus high-frequency performance. Generally, when faced with an aircraft exhibiting some PIO tendencies, the pilot will "back off" and sacrifice low frequency performance to minimize rms error due to any excessive resonance in the system. This statement is justified by correlating maximum lowfrequency "droop" with the pilot's comments (Refer to Tables 4.4, 4.5, 4.6). Upon comparing cases with neighboring bandwidth frequencies, the ones found to exhibit a lower droop automatically had higher error rms values and pilot comments indicating some overshoot and PIO problems. This "delicate" strategy of avoiding lightly damped oscillation (or PIO's) will always be the result obtained from the OCM due to the guaranteed stability of this optimal solution -- or it will always use the "best" piloting strategy. Alternatively, one could argue that PIO's are the results of a sub-optimal piloting strategy, and in particular, this usually means that the pilot's "gain" is too high (above the "optimal").

On the hypothesis that the optimal (OCM) pilot is sacrificing low frequency performance, suppose an additional gain is added in the "forward path" for example, to raise the closed-loop droop to try to achieve a higher level of tracking performance. Typically, the droop of the configurations analyzed, ranged from -.3 to $-7.0 \mathrm{~dB}$. Adjusting the additional forward path gain to achieve

$$
\left|\frac{\theta}{\theta_{c}}\right|>-.6 \mathrm{~dB} \text { for } \omega \leq \mathrm{BW}
$$


Table 4.4 Summary of Results Obtained for the 8 Basic Short-Period Configurations

\begin{tabular}{|c|c|c|c|c|c|c|c|}
\hline Conf. & $\begin{array}{l}\text { Pilot } \\
\text { Rating }\end{array}$ & $\begin{array}{l}\text { Bandwidth } \\
(\mathrm{rad} / \mathrm{sec})\end{array}$ & $\begin{array}{l}\text { Droop } \\
(\mathrm{dB})\end{array}$ & $\begin{array}{l}\left|\frac{\theta}{\theta_{c}}\right|_{\text {max }} \\
(d \mathrm{~dB})\end{array}$ & $\begin{array}{l}\text { OCM } \\
(\text { deg })\end{array}$ & $\begin{array}{l}{ }_{\mathrm{pc}} \\
(\mathrm{deg})\end{array}$ & $\begin{array}{c}{ }_{\varepsilon} \\
\left({ }^{\text {deg }}\right)\end{array}$ \\
\hline 10 & $3-5$ & 3.267 & -.4359 & 1.834 & -6.010 & +49.52 & .7250 \\
\hline 20 & 2.5 & 3.675 & -.5416 & 1.244 & -55.61 & +6.68 & .7226 \\
\hline $3 A$ & $4-5$ & 3.472 & -.6543 & .6765 & -87.73 & -28.79 & .7850 \\
\hline $4 A$ & 5.5 & 3.700 & -.8324 & 10.17 & -73.07 & -10.36 & .8714 \\
\hline $5 A$ & $5-7$ & 3.403 & -.9909 & 18.21 & -85.59 & -27.80 & .9511 \\
\hline $6 C$ & 4.0 & 3.322 & -.4172 & 1.250 & -22.44 & +34.01 & .7244 \\
\hline $7 C$ & $1.5-4$ & 3.619 & -.4272 & .7662 & -63.58 & -2.21 & .6828 \\
\hline $8 A$ & $4-5$ & 3.573 & -.4690 & .6460 & -85.26 & -25.64 & .6968 \\
\hline
\end{tabular}


Table 4.5 Summary of Results Obtained for Configurations of Group ?

\begin{tabular}{|c|c|c|c|c|c|c|c|}
\hline Conf. & $\begin{array}{l}\text { Pilot } \\
\text { Rating }\end{array}$ & $\begin{array}{l}\text { Bandwidth } \\
(\mathrm{rad} / \mathrm{sec})\end{array}$ & $\begin{array}{r}\text { Droop } \\
(\mathrm{dB})\end{array}$ & ${ }^{\left|\frac{\theta}{\theta}\right|_{\text {max }}}(\mathrm{dB})$ & $\begin{array}{l}\text { OCM } \\
\text { (deg) }\end{array}$ & $\begin{array}{c}\downarrow_{p c} \\
(\mathrm{deg})\end{array}$ & $\begin{array}{c}\sigma_{\varepsilon} \\
(\mathrm{deg})\end{array}$ \\
\hline $1 \mathrm{~A}$ & $4-6$ & 3.525 & -.7354 & 7.186 & -24.15 & +35.66 & .7421 \\
\hline $1 \mathrm{~B}$ & 3.5 & 3.488 & -.4910 & 1.861 & -26.91 & +32.29 & .7002 \\
\hline IC & $3.5-5$ & 3.057 & -.6316 & 4.843 & -17.71 & +34.32 & .8373 \\
\hline 10 & $3-5$ & 3.267 & -.4359 & 1.834 & -6.010 & +49.52 & .7250 \\
\hline IE & 6.0 & 2.842 & -.5394 & 3.585 & 14.74 & +63.17 & .8831 \\
\hline IF & 8.0 & 2.659 & -.4652 & 5.028 & 33.34 & +78.70 & .9537 \\
\hline $1 G$ & 8.5 & 2.308 & +.0056 & 4.690 & 50.51 & +89.96 & 1.0680 \\
\hline
\end{tabular}


Table 4.6 Summary of Results Obtained for Configurations of Group 2

\begin{tabular}{|c|c|c|c|c|c|c|c|}
\hline Conf. & $\begin{array}{r}\text { Pilot } \\
\text { Rating }\end{array}$ & $\begin{array}{l}\text { Bandwidth } \\
(\mathrm{rad} / \mathrm{sec})\end{array}$ & $\begin{array}{r}\text { Droop } \\
(\mathrm{dB})\end{array}$ & $\begin{array}{l}\left|\frac{\theta}{\theta_{C}}\right|_{\max } \\
(d B)\end{array}$ & $\begin{array}{l}\text { OCM } \\
\text { (deg) }\end{array}$ & $\begin{array}{l}{ }_{\mathrm{pc}} \\
\text { (deg) }\end{array}$ & $\begin{array}{c}\sigma_{\varepsilon} \\
(\operatorname{deg})\end{array}$ \\
\hline $2 A$ & 4.5 & 3.778 & -.7581 & 4.967 & -73.82 & -9.83 & .8081 \\
\hline $2 B$ & $4-6$ & 3.320 & -.8644 & 11.37 & -64.72 & -8.31 & .9150 \\
\hline $2 C$ & 3.0 & 3.783 & -.5898 & 1.200 & -70.31 & -6.24 & .7335 \\
\hline 20 & 2.5 & 3.675 & -.5416 & 1.244 & -55.61 & +6.68 & .7226 \\
\hline $2 E$ & 4.0 & 3.369 & -.6024 & 3.278 & -45.69 & +11.54 & .7960 \\
\hline $2 F$ & 3.0 & 3.201 & -.6045 & 3.901 & -32.95 & +21.48 & .8231 \\
\hline $2 G$ & 7.0 & 2.854 & -.7423 & 9.250 & -25.67 & +22.97 & .9513 \\
\hline $2 \mathrm{H}$ & $5-6$ & 2.998 & -.5282 & 2.504 & -13.87 & +37.18 & .8391 \\
\hline $2 I$ & 8.0 & 2.673 & -.6639 & 6.360 & -6.754 & +38.84 & .9768 \\
\hline $2 \mathrm{~J}$ & 6.0 & 2.806 & -.0498 & 3.876 & 7.010 & +54.84 & .8617 \\
\hline
\end{tabular}


produced resonant peaks comparable to those in Neal-Smith and therefore exposed those PIO prone configurations with this parameter. This procedure may sound reminiscent of the original approach, but only one degree of freedom exists -- that of "DC" gain adjustment. This was accomplished as an integral part of the computer-based analysis in the following way.

1) Scan the magnitude of the closed-10op droop for minimum $\left|\frac{\theta}{\theta^{c} c_{\omega=\omega_{\min }}}\right| ; 0<\omega_{\min }<B w$ for $\omega_{\min }$ (frequency at the droop)

2) At $\omega_{\min }$ record the open-100p $\frac{\theta}{\varepsilon}(s) \mathrm{pilot} /$ vehicle frequency response as the complex number $\alpha+i_{B}$.

3) Find additional pilot gain $K_{a}$ to produce $-.6 \mathrm{~dB}$ droop at $\omega_{\min }$ by

$$
K_{a}=\frac{-B+\sqrt{B^{2}-4 A C}}{2 A}
$$

where

$$
\begin{aligned}
& A=\left(1-(\gamma)^{2}\right)\left(\alpha^{2}+\beta^{2}\right) \\
& B=-2(\gamma)^{2} \\
& C=-(\gamma)^{2} \\
& \gamma=0.9447
\end{aligned}
$$

In $d B \quad K_{a}=20 \log \left(K_{a}\right)$

4) Add $K_{a}$ to the open-loop frequency response $\frac{\theta}{\varepsilon}(s)$ and re-evaluate the closed-loop frequency response

$$
\frac{\theta}{\theta_{c}}(s)=\frac{\frac{\theta}{\varepsilon}(s)}{1+\frac{\theta}{\varepsilon}(s)} \quad s=j \omega
$$


5) Check droop and scan for the corrected

$$
\left|\frac{\theta}{\theta_{c}}\right|_{\max } .
$$

Some of the benefits of using the OCM model are evident in the results of Figure [4.5] corresponding to the configurations sumnarized in Appendix C. Of particular interest are those vehicles the Neal and Smith approach failed to place in the correct "areas". In one instance, configuration $8 \mathrm{~A}$ appeared in the level one area, although it received a level 2 rating. We were able to not only identify it properly as level 2 , but placed the aircraft next to another configuration ( $3 A$ ) with different short period characteristics, but sharing the same pilot comments and pilot ratings (4-5). Other examples, such as level-3 configuration $2 G$ were incorrectly placed in the level 2 area by NealSmith. Once again, the OCM approach predicted a PIO problem serious enough to warrant a level 3 rating. In cases correctly rated by Neal and Smith, agreement was aimost always attained by the OCM approach. Configurations $2 C, 7 C$, and $2 D$ were all given high marks in acquiring the target, which exemplifies the level I rating predicted by both methods. In only two cases evaluated (2E, $2 F)$ did the OCM method yield marginal results. These configurations are on the level 1 - level 2 boundary, and only one rating data point was obtained for each configuration. 
CHAPTER 5

SUMMARY

\subsection{Summary and Conclusions}

The main objective of this research has been to incorporate the benefits of the optimal control model with the basic framework of Neal and Smith's "pilot-in-the-loop" analysis to effectively create a more powerful tool for predicting pilot opinion rating. Indeed, by minimizing many of the shortcomings of the so-called "classical" model, and by preserving the simplicity found in the closed-loop frequency response, the OCM has led to an improvement in a method handicapped by the validity of the pilot model and the difficulty in obtaining it.

Consider then, the following benefits resulting from the new approach.

1) The OCM has the capacity to better represent complex pilot compensation likely to be present in the control of high-order dynamics, in contrast to being restricted to the lead-lag compensation of Neal and Smith.

2) Use of the OCM reflects more correctly the actual experimental situation, incorporating the effects of human factors in modeling the tracking task.

3) The linear time invariant structure of the OCM makes it equally suitable for analysis in the frequency domain, such as Neal and Smith. 
4) Use of the OCM gives the new method more flexibility over the Neal and Smith method in that more complicated tasks involving additional loop closures can be tackled easily in its state space format.

5) The Neal-Smith pilot model parameters $\left(K_{p}, T_{p_{1}}, T_{p_{2}}\right)$ have to be chosen to meet a certain standard of low-frequency performance whereas the OCM "automatically" leads to the optimal solution to do the best job (of task execution) possible subject to human limitations.

6) The task performance in terms of droop and bandwidths now varies from configuration to configuration.

7) More importantly, the new approach eliminates the critical task of pre-selecting bandwidth in favor of selecting more fundamental physiological limitations such as neuromotor lag.

8) Aiso, the analysis demonstrated that an inability to obtain suitable bandwidth analytically correlates well with the bandwidthrelated problems (i.e. large initial response delays) encountered in flight.

In conclusion, the method provides a promising alternative to the classical Neal and Smith "pilot-in-the-10op" analysis, with what is considered a better pilot modeling procedure.

\subsection{Areas of Further Study}

To reflect the oscillatory tendencies experienced by the pilot, a "DC" gain, adjusted to reach a "suboptimal" Tevel of performance, was added to the OCM results. Just how valid this step is relies on its underlying assumption; that a trade-off exists between attaining lowfrequency performance and minimizing oscillatory tendencies. This 
assumption can only be scrutinized with more piloted simulations over a wide range of FCS/aircraft dynamics.

As a confirmation of this study, one may consider applying the method to more complex task such as approach and landing. Smith's LAHOS (Landing and Approach of Higher Order Systems) Report [7] stated that extrapolating the original closed-loop Neal/Smith criterion failed to reveal pitch attitude problems experienced in landing. Perhaps neglecting the additional loop closures required to perform the approach and landing task was the classical approach's undoing.

of course, this is not a problem for the OCM. What is a problem and requires further investigation is how to select the cost functional weightings representative of the pilot's strategy to perform such a task. 


\section{LIST OF REFERENCES}

[1] Neal, T.P. and Smith, R.E., An In-Flight Investigation to Develop Control System Design Criteria for Fighter Airplanes. AFFDL-TR-70-74, Vo1. I, December (1970).

[2] Kleinman, D.L., Baron, S. and Levison, W.H., "An Optimal Control Model of Human Response Part I: Theory and Validation". Automatica, Vo1.6, pp. 357-369, Pergamon Press, (1970).

[3] Kleinman, D.L., "Optimal Control of Linear Systems with Time Delay and Observation Noise", IEEE Trans. on Auto. Control, $A C-74$, October (1979).

[4] Wonham, W.M., "On the Separation Theorem of Stochastic Control", SIAM J. Control, Vol. 6, pp. 312-326, 1968.

[5] Curry, R.E., Hoffman, W.C. and Young, L.R., Piloting Modeling for Manned Simulation, Vols. I and II, AFFDL-TR-76-124, Wright Patterson AFB, Ohio, (1976).

[6] Schmidt, D.K., Multivariable Closed-Loop Analysis and Flight Control Synthesis for Air-to-Air Tracking. AFOSR-79-0042, June (1980).

[7] Smith, Ro E., Effects of Control System Dynamics on Fighter Approach and Landing Longitudinal Flying Qualities, Vol. T, AFFDL-TR-78-122, Wright-Patterson AFB, Ohio, (1978). 
APPENDIX A

SIMPLIFYING THE TOTAL COST OF SECTION 3.2

Problem: Reduce the total cost of Section 3.2,

$$
\begin{aligned}
J(\bar{u})= & \operatorname{tr}(Q \bar{\Sigma})+\operatorname{tr}\left(Q \int_{0}^{\tau} e^{A \xi} \bar{\Sigma} C^{1} V^{-1} C \bar{\Sigma} e^{A^{\prime} \xi} d \xi\right) \\
& +\operatorname{tr}\left(K e^{A \tau} \bar{\Sigma} C^{\prime} V^{-1} C \bar{\Sigma} e^{A^{\prime} \tau}\right)
\end{aligned}
$$

to the form Kleinman developed in Ref [3].

Solution: To simplify this expression of total cost, subsitute the known steady-state equality

$$
\bar{\Sigma} C^{\prime} V^{-1} C \bar{\Sigma}=A \bar{\Sigma}+\bar{\Sigma} A^{\prime}+W
$$

into E.q. (A.1)

$$
\begin{aligned}
J(\bar{u}) & =\operatorname{tr}(Q \bar{\Sigma})+\operatorname{tr}\left(Q \int_{0}^{\tau} e^{A \xi} W e^{A^{\prime} \xi} d \xi\right)+\operatorname{tr}\left(K e^{A \tau} W e^{A^{\prime} \tau}\right) \\
& +\operatorname{tr}\left(Q \int_{0}^{\tau} e^{A \xi}\left(A \bar{\Sigma}+\bar{\Sigma} A^{\prime}\right) e^{A^{\prime} \xi} d \xi\right) \\
& +\operatorname{tr}\left(K e^{A \tau}\left(A \bar{\Sigma}+\bar{\Sigma} A^{\prime}\right) e^{A^{\prime} \tau}\right) .
\end{aligned}
$$

At first glance, this expression looks worse than the previous one, but take heart, for some sweat and algebraic matrix manipulation will bring the last two terms into a cleaner form. First, expand the integral of the fourth term to 


$$
\begin{aligned}
& \int_{0}^{\tau} e^{A \xi}\left(A \bar{\Sigma}+\bar{\Sigma} A^{\prime}\right) e^{A^{\prime} \xi} d \xi= \\
& \quad \int_{0}^{\tau} e^{A \xi} A \bar{\Sigma} e^{A^{\prime} \xi} d \xi+\int_{0}^{\tau} e^{A \xi} \bar{\Sigma} A^{\prime} e^{A^{\prime} \xi} d \xi
\end{aligned}
$$

Integrating the second term of Eq. $(A .4)$ by parts

$$
\int_{0}^{\tau} e^{A \xi} \vec{\Sigma} A^{\prime} e^{A^{\prime} \xi} d \xi=\left.e^{A \xi} \bar{\Sigma} e^{A^{\prime} \xi}\right|_{0} ^{\tau}-\int_{0}^{\tau} e^{A \xi} A \bar{\Sigma} e^{A^{\xi} \xi} d \xi
$$

simplifies the expression to

$$
\begin{array}{r}
\int_{0}^{\tau} e^{A \xi}\left(A \bar{\Sigma}+\tilde{\Sigma} A^{B}\right) e^{A^{B} \xi} d \xi= \\
e^{A \tau} \bar{\Sigma} e^{A^{\prime} \tau}-\bar{\Sigma}
\end{array}
$$

Furthermore, taking advantage of the commutative property that exists between matrices $A$ and $e^{A t}$, and using the trace identity,

$$
\operatorname{tr}(N M)=\operatorname{tr}(M N) \text { when } N(n \times m) \text { and } M(m \times n)
$$

the last term of Eq. (A.3) can be directly expressed as a combination of

$$
\begin{aligned}
\operatorname{tr}\left(K e^{A \tau} \bar{\Sigma} A^{\prime} e^{A^{\prime} \tau}\right) & =\operatorname{tr}\left(K e^{A \tau} \bar{\Sigma} e^{\left.A^{\prime} \tau^{\prime} A^{\prime}\right)}\right. \\
& =\operatorname{tr}\left(A^{\prime} K e^{A \tau \bar{\Sigma}} e^{A^{\prime} \tau}\right)
\end{aligned}
$$

and

$$
\operatorname{tr}\left(K e^{A \tau} A \bar{\Sigma} e^{A^{\prime} \tau}\right)=\operatorname{tr}\left(K A e^{A \tau} \bar{\Sigma} e^{A^{\prime} \tau}\right)
$$

to give the desired form 


$$
\operatorname{tr}\left(K e^{A \tau}\left(A \bar{\Sigma}+\bar{\Sigma} A^{\prime}\right) e^{A^{\prime} \tau}\right)=\operatorname{tr}\left(\left(K A+A^{\prime} K\right) e^{A \tau} \bar{\Sigma} e^{A^{\prime} \tau}\right)
$$

This expression combined with Eq. (A.6) reduces the total cost to

$$
\begin{aligned}
J(\bar{u})= & \operatorname{tr}\left(Q \int_{0}^{\tau} e^{A \xi} W e^{A^{\prime} \xi} d \xi\right)+\operatorname{tr}\left(K e^{A \tau} W e^{A^{\prime} \tau}\right) \\
& +\operatorname{tr}\left(\left(K A+A^{\prime} K+Q\right) e^{A \tau} \bar{\Sigma} e^{A^{\prime} \tau}\right)
\end{aligned}
$$

Observing that the matrix Ricatti equation may be expressed as

$$
K A+A^{\prime} K+Q=L^{* 1} R L^{*}=L^{* 1} R^{1 / 2} R^{1 / 2} L^{*}
$$

the third term of Eq. (A.10) collapses neatly to $\operatorname{tr}\left(L^{* 1} R^{1 / 2} R^{1 / 2} L^{*} e^{A \tau_{\bar{\Sigma}}}\right.$ $\left.e^{A^{\prime} \tau}\right)$. This term is brought into final form by using the trace identity, previously cited.

$$
\begin{aligned}
\operatorname{tr}\left(L^{*}{ }_{R}{ }^{1 / 2} R^{1 / 2} L^{*} e^{A} \tau_{\bar{\Sigma}} e^{A^{\prime} \tau}\right) & =\operatorname{tr}\left(R^{1 / 2} L^{*} e^{A} \tau_{\bar{\Sigma}} e^{\left.A^{\prime} \tau_{L}{ }^{* 1}{ }_{R}{ }^{1 / 2}\right)}\right. \\
& =\operatorname{tr}\left(L_{e} e^{\bar{\Sigma} L^{\prime}} e^{\prime}\right)
\end{aligned}
$$

where $L_{e}=R^{1 / 2} L^{*} e^{A \tau}$

Making the appropriate substitutions, the total cost is

$$
\begin{aligned}
J\left(\bar{u}^{*}\right)= & \operatorname{tr}\left(Q \int_{0}^{\tau} e^{A \xi} W e^{A^{\prime} \xi} d \xi\right)+\operatorname{tr}\left(K e^{A \tau} W e^{A^{\prime} \tau}\right) \\
& +\operatorname{tr}\left(L_{e} \bar{\Sigma} L_{e}{ }^{\prime}\right),
\end{aligned}
$$

the same as Kleinman developed in Ref. [3]. 


\section{APPENDIX B \\ FORMULATION OF THE STATE COVARIANCE MATRIX FOR SECTION (3.2)}

The simplest method of producing the covariance matrix follows directly from noting the actual state $\bar{x}(t)$ is the sum of two orthogonal components

$$
\bar{x}(t)=\ddot{x}(t)+\ddot{e}(t)
$$

with $\bar{e}(t)$, the estimation error and $\hat{\bar{x}}(t)$, the least-mean-square estimate of $\bar{x}(t)$ where $t$ replaces $t-\tau$ since in this steady-state development $t$ tends to infinity. Equation (B. I) can be expanded further by recalling that $\bar{x}(t)$ is also the sum of orthogonal components as a result of the prediction process. Thus

$$
\hat{\bar{x}}(t)=\bar{y}(t)+\bar{e}_{p}(t)
$$

with $\bar{e}_{p}(t)$, the prediction error and $\bar{\gamma}(t)$, the least-mean-square prediction of $\hat{\bar{x}}(t)$. Since $\hat{\bar{x}}(t)$ is orthogonal to the estimation error $\bar{e}(t)$, the orthogonal decomposition of $\hat{\bar{x}}(t)$ is aiso orthogonal to $\bar{e}(t)$. Hence, the following relationship hold

$$
\begin{aligned}
& \lim _{t \rightarrow \infty} E\left\{\bar{e}(t) \bar{e}_{p}^{\prime}(t)\right\}=0 \\
& \lim _{t \rightarrow \infty} E\left\{\bar{e}(t) \bar{\gamma}^{\prime}(t)\right\}=0
\end{aligned}
$$


and of course

$$
\lim _{t \rightarrow \infty} E\left\{e_{p}(t) \bar{\gamma}^{1}(t)\right\}=0
$$

Thus, the steady-state, state covariance can be expanded, accordingly

$$
\begin{aligned}
\lim _{t \rightarrow \infty} E\left\{\bar{x}(t) \bar{x}^{\prime}(t)\right\}+\lim _{t \rightarrow \infty} E\left\{\bar{\gamma}(t) \bar{\gamma}^{\prime}(t)\right\} \\
+E\left\{\bar{e}_{p}(t) \bar{e}_{p}^{\prime}(t)\right\}+E\left\{\bar{e}(t) \bar{e}^{\prime}(t)\right\}
\end{aligned}
$$

with the first term, derivable from the solution of Eq. (3.44)

$$
\bar{\gamma}(t)=e^{\bar{A} t-}(0)+\int_{0}^{t} e^{\bar{A}(t-\sigma)} \tilde{w}_{2}(\sigma) d \sigma
$$

with $\ddot{A}=A-B L^{*}$ and $\bar{\gamma}(0)=0$. Thus

$$
\begin{aligned}
E\left\{\bar{\gamma}(t) \bar{\gamma}^{\prime}(t)\right\} & =\int_{0}^{t} e^{\bar{A}}(t-\sigma) e^{A \tau} \tilde{W} e^{A^{\prime} \tau} e^{\bar{A}^{\prime}(t-\sigma)} d \sigma \\
& =\int_{0}^{t} e^{\bar{A}(t-\sigma))} e^{A \tau} \bar{\Sigma} C^{\prime} v^{-1} C \bar{\Sigma} e^{A^{\prime} \tau} e^{\bar{A}^{\prime}(t-\sigma)} d \sigma
\end{aligned}
$$

Letting $t$ go to infinity,

$$
\lim _{t \rightarrow \infty} E\left\{\bar{\gamma}(t) \bar{\gamma}^{\prime}(t)\right\}=\int_{0}^{\infty} e^{\bar{A} \sigma} e^{A \tau} \bar{\Sigma} C^{\prime} V^{-1} C \bar{\Sigma} e^{A^{\prime} \tau} e^{\bar{A}^{\prime} \sigma} d \sigma
$$

The covariance of the prediction error has already been evaluated in finding the total cost. Thus,

$$
\lim _{t \rightarrow \infty} E\left\{\bar{e}_{p}(t) \bar{e}_{p}{ }^{\prime}(t)\right\}=\int_{0}^{\tau} e^{A \xi} \tilde{W}^{A}{ }^{\prime} \xi \xi \xi
$$




$$
=\int_{0}^{\tau} e^{A \xi} \bar{\Sigma} C^{\prime} V^{-1} C \bar{\Sigma} e^{A^{\prime} \xi} d \xi
$$

and

$$
=e^{A \tau} \Sigma e^{A^{\prime} \tau}-\Sigma+\int_{0}^{\tau} e^{A \xi} W e^{A} \xi d \xi
$$

from Eq. (3.39), Eqs. (A.1) - (A.6). Lastly, the third term of Eq. (B.4) is simply the definition of $\bar{\Sigma}$. Hence, summing the three terms

$$
\begin{aligned}
E\left\{\tilde{x}(t) \cdot \dot{x}^{\prime}(t)\right\}=e^{A \tau} \bar{\Sigma} e^{A^{\prime} \tau}+\int_{0}^{\tau} e^{A \xi} W e^{A^{\prime} \xi} d \xi \\
+\int_{0}^{\infty}\left(e^{\bar{A} \sigma} e^{A \tau} \bar{\Sigma} C^{\prime} V^{-1} C \bar{\Sigma} e^{A^{\prime} \tau} e^{\bar{A}^{\prime} \sigma}\right) d \sigma
\end{aligned}
$$

reproduces Kleinman's closed-form expression of the state covariance matrix. 


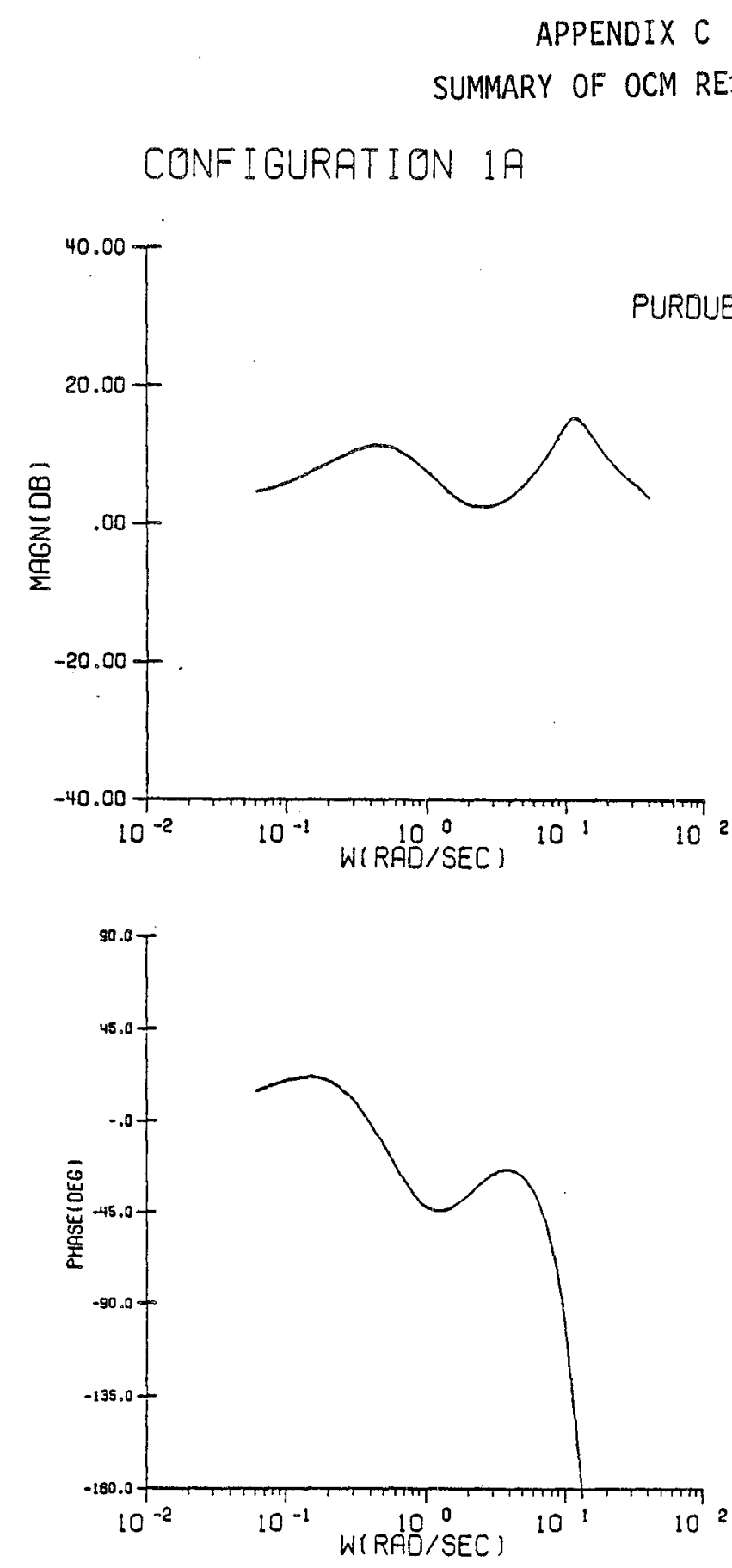

Figure C.1 Configuration 1.A/Pi lot Frequency Response 
CONFIGURATION $1 \mathrm{~A}$

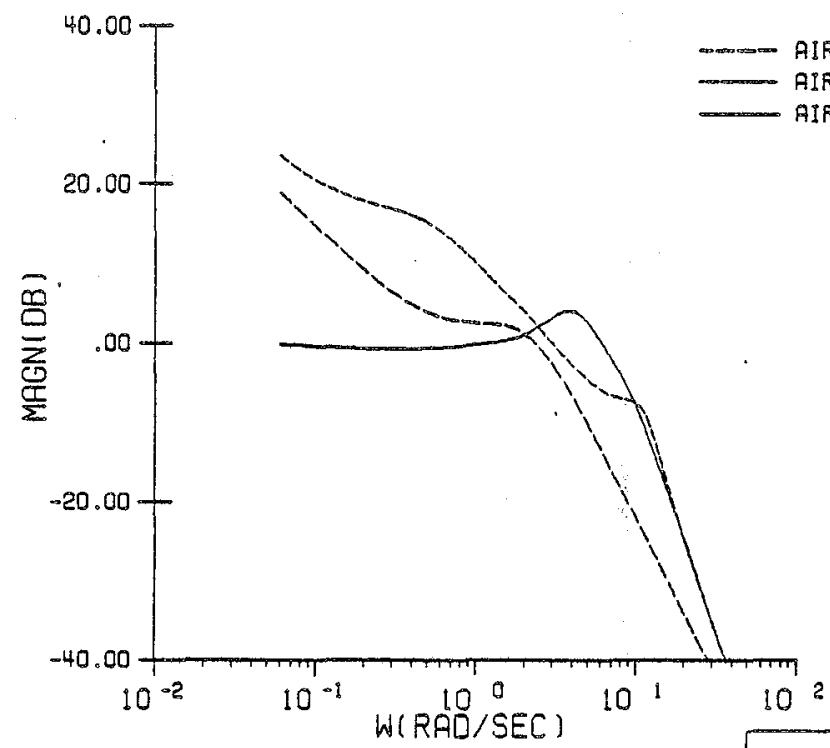

BANDWIOTHE $=3.53$ RAO/SEC

PYLOT COMPENSATION $=-24.15$ DEG

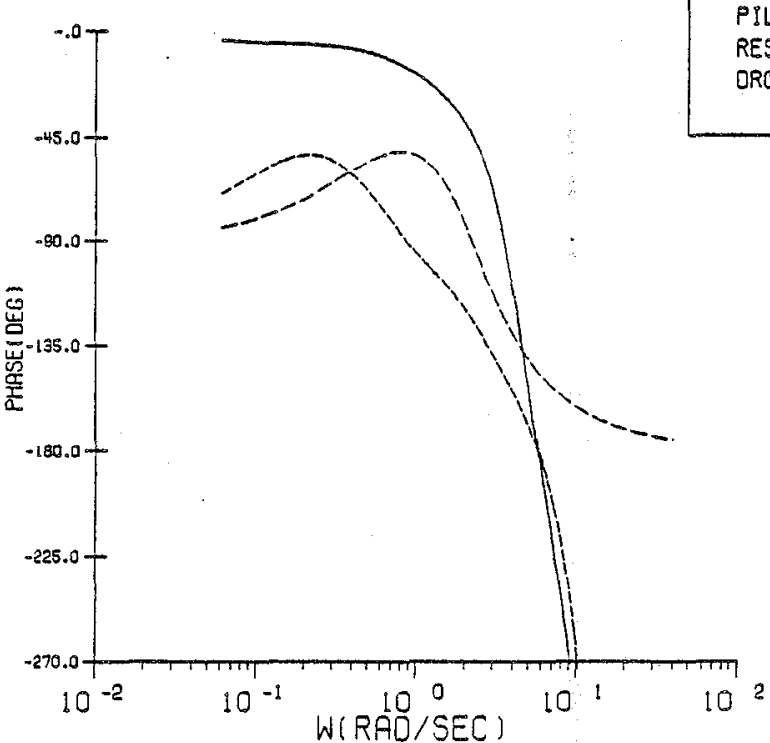

Figure C.2 Configuration $1 \mathrm{~A} /$ System Frequency Response 
CONFIGURATION $1 \mathrm{~A}$

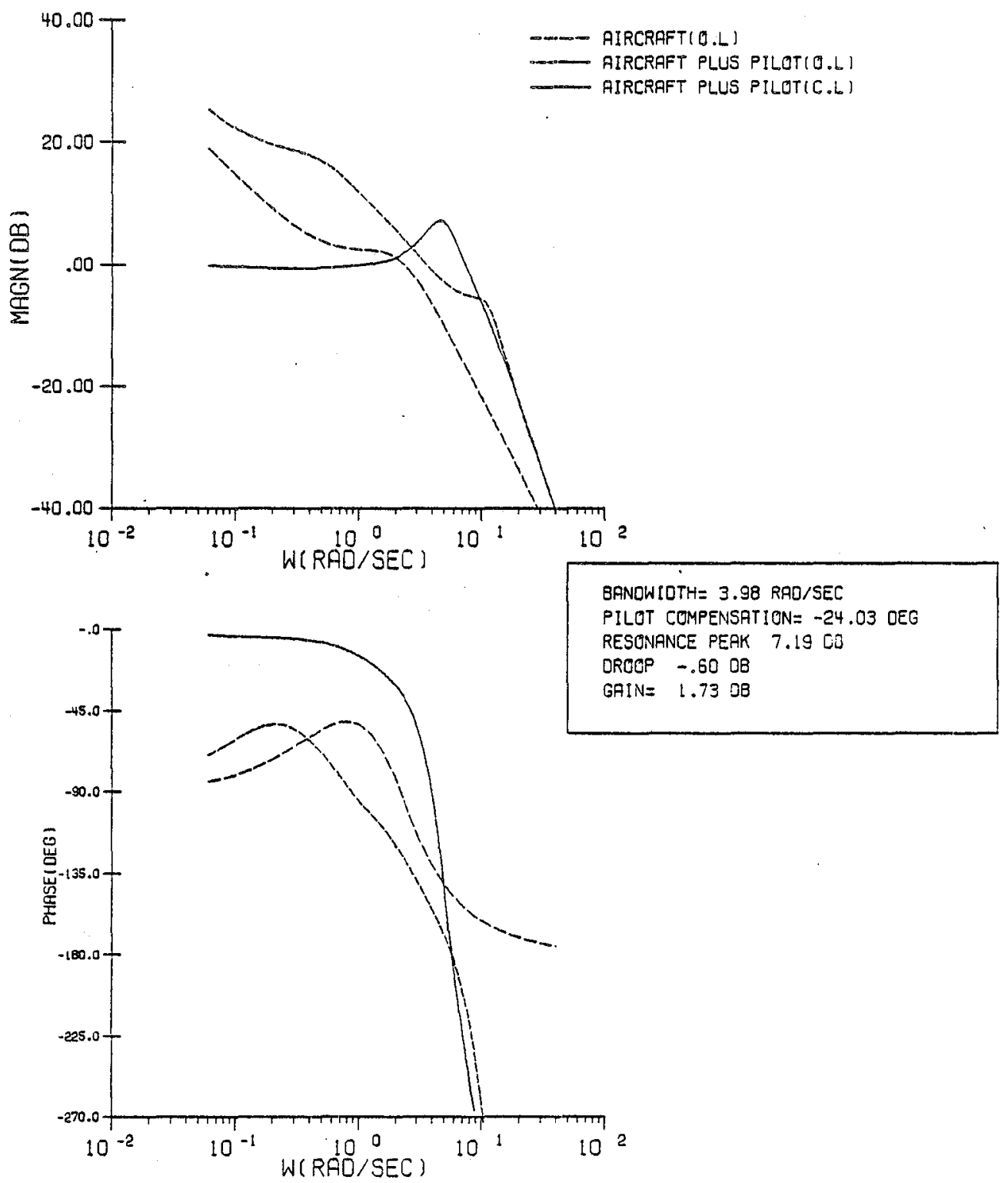

Figure C.3 Configuration $\mathrm{TA} /$ Corrected System Frequency Response 
CONFIGURATION $1 \mathrm{~B}$
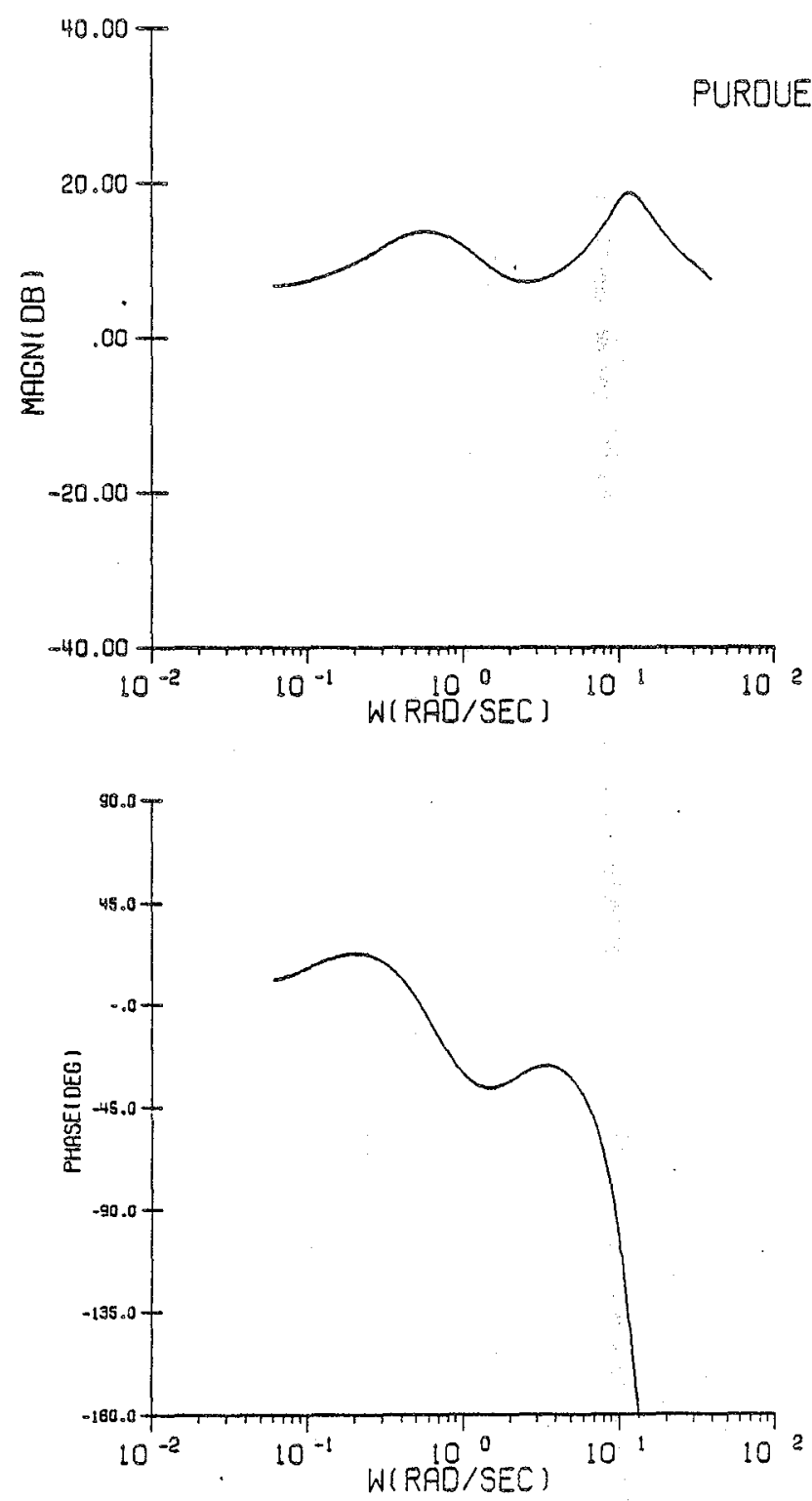

Figure C.4 Configuration 1B/Pilot Frequency Response 
CONF IGURATION $1 B$

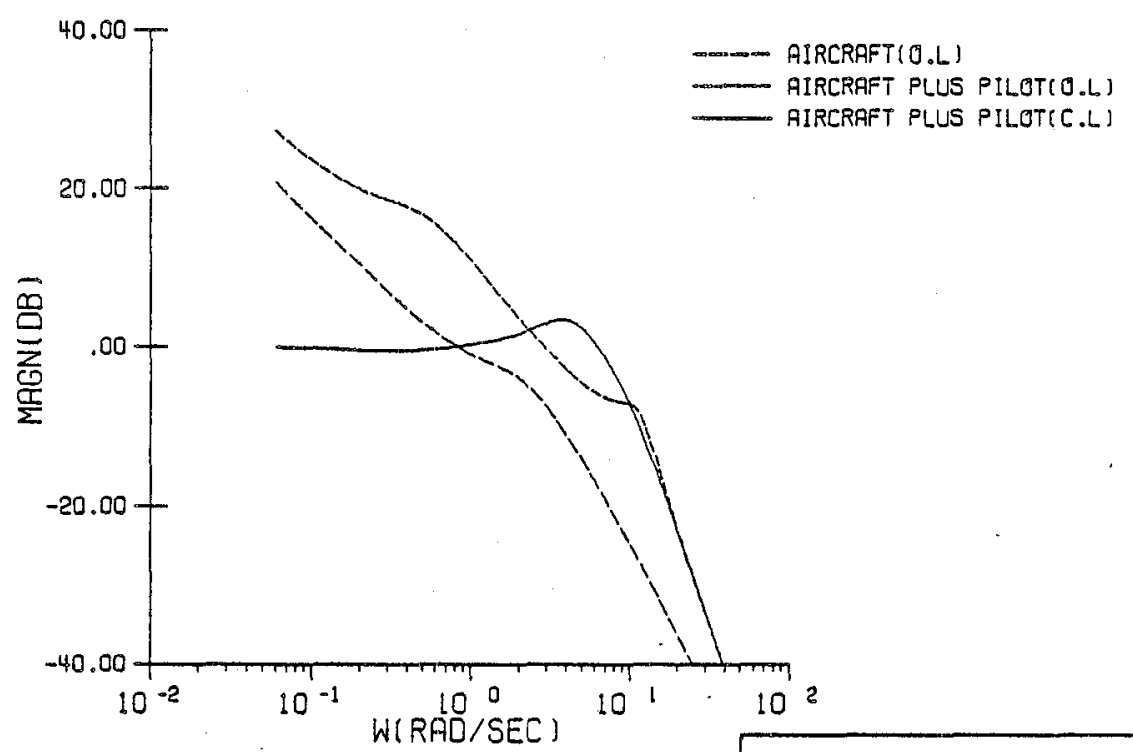

BANOWIDTH $=3.49$ RAO/SEC

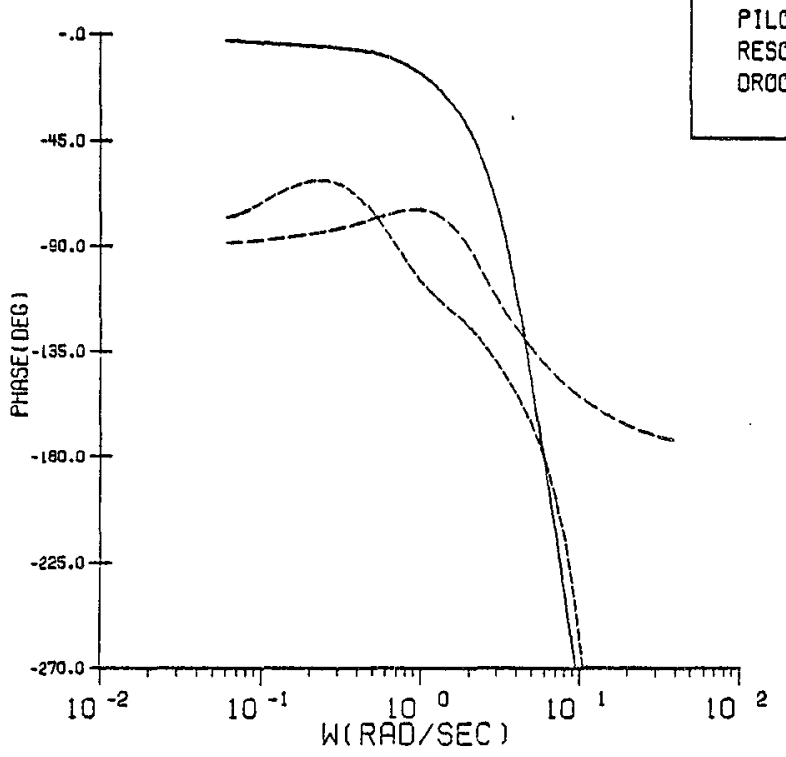

Figure C.5 Configuration $\mathrm{B} / \mathrm{B}$ System Frequency Response 


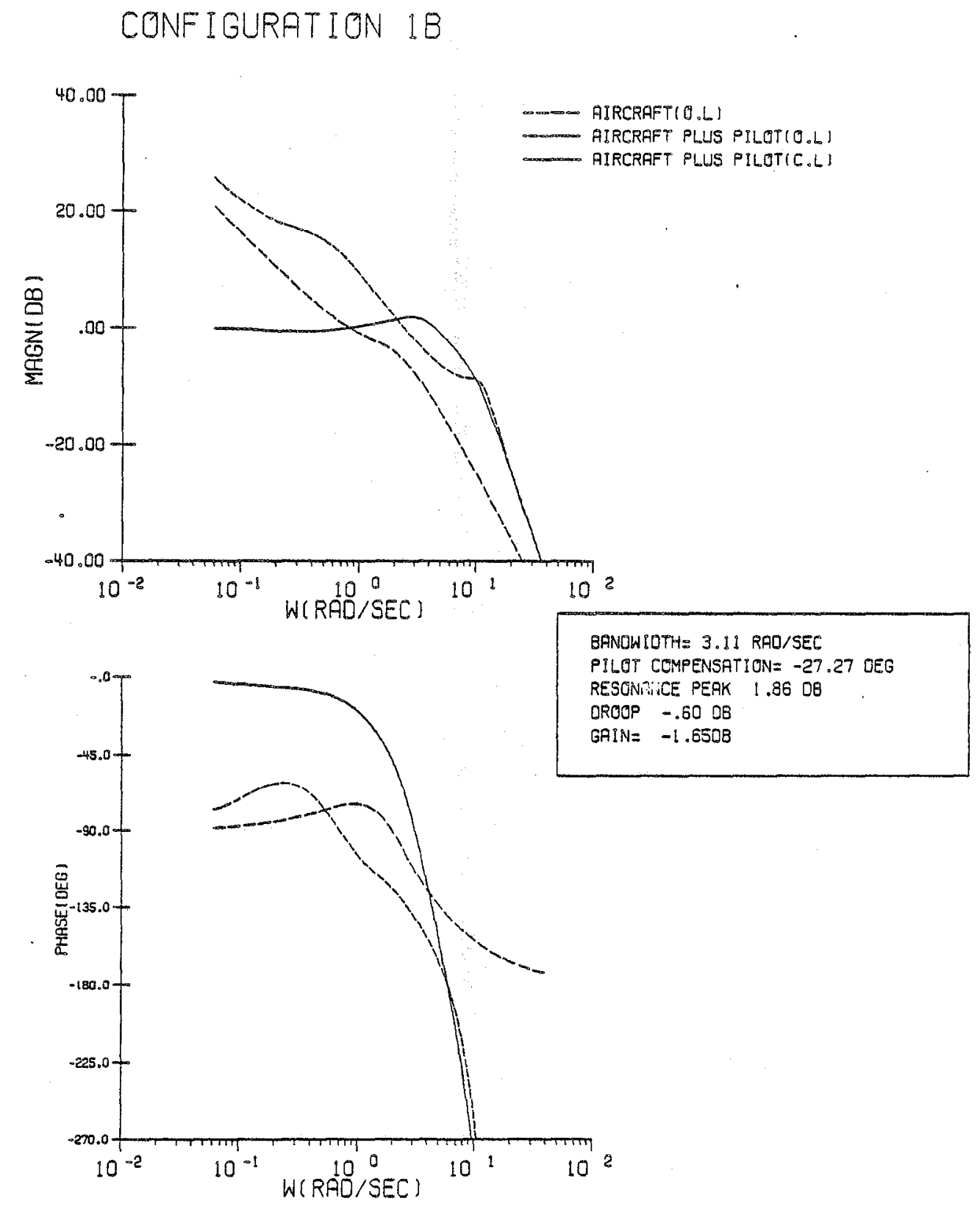

Figure C.6 Configuration 1B/Corrected System Frequency Response 
CONFIGURATION $1 \mathrm{C}$
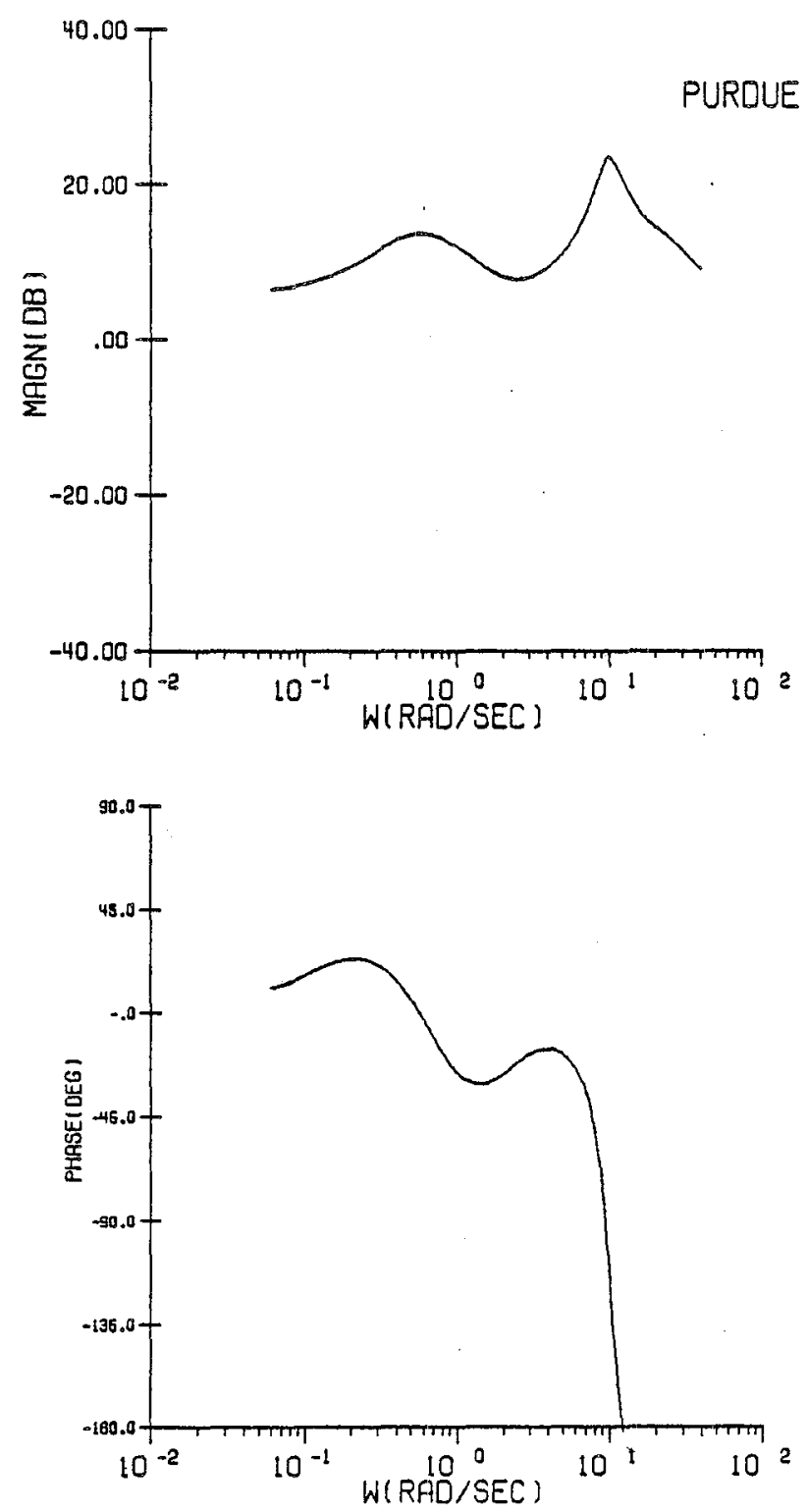

Figure C.7 Configuration $1 C / P i$ lot Frequency Response 


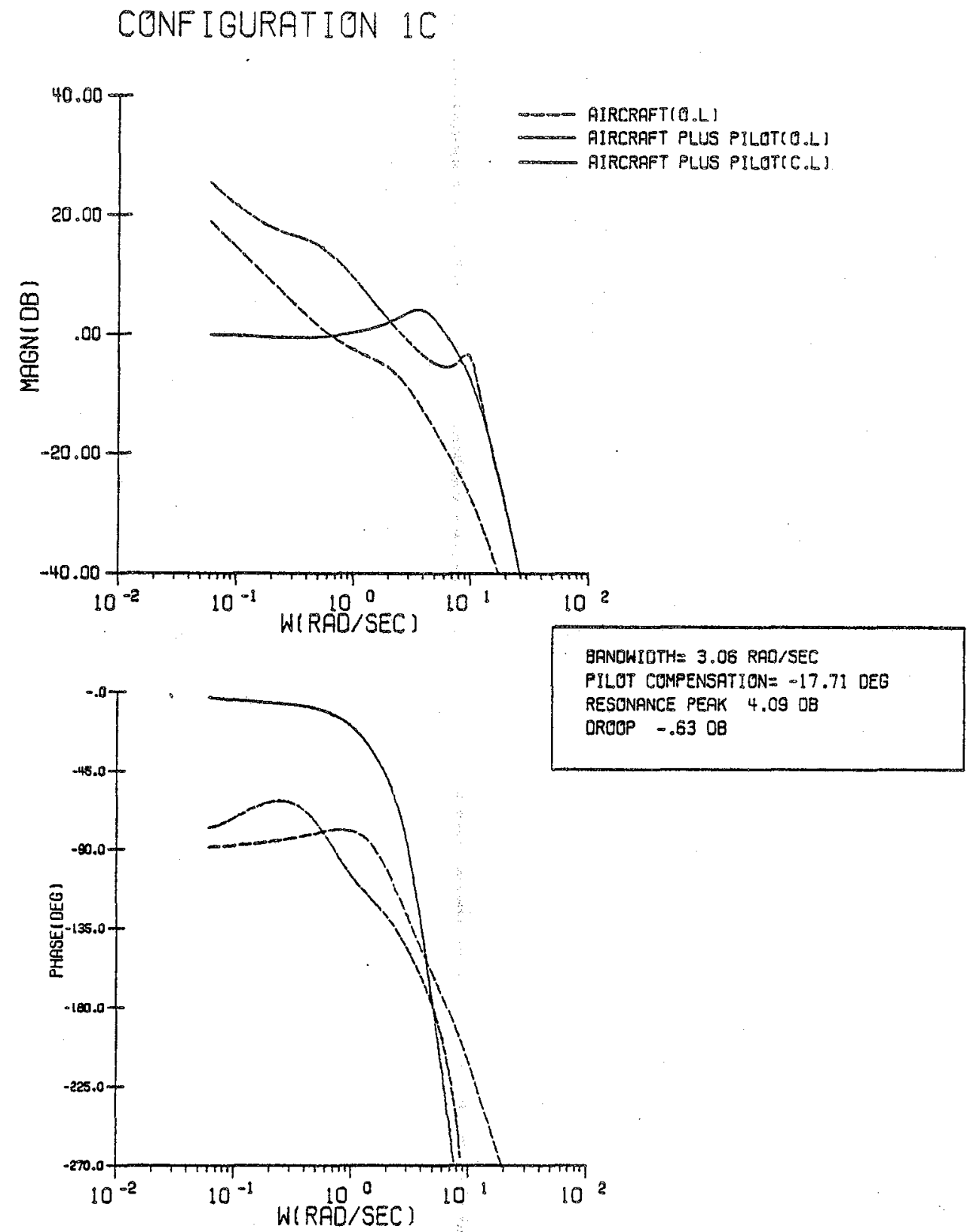

Figure C.8 Configuration 1C/System Frequency Response 
CONFIGURATION $1 \mathrm{C}$

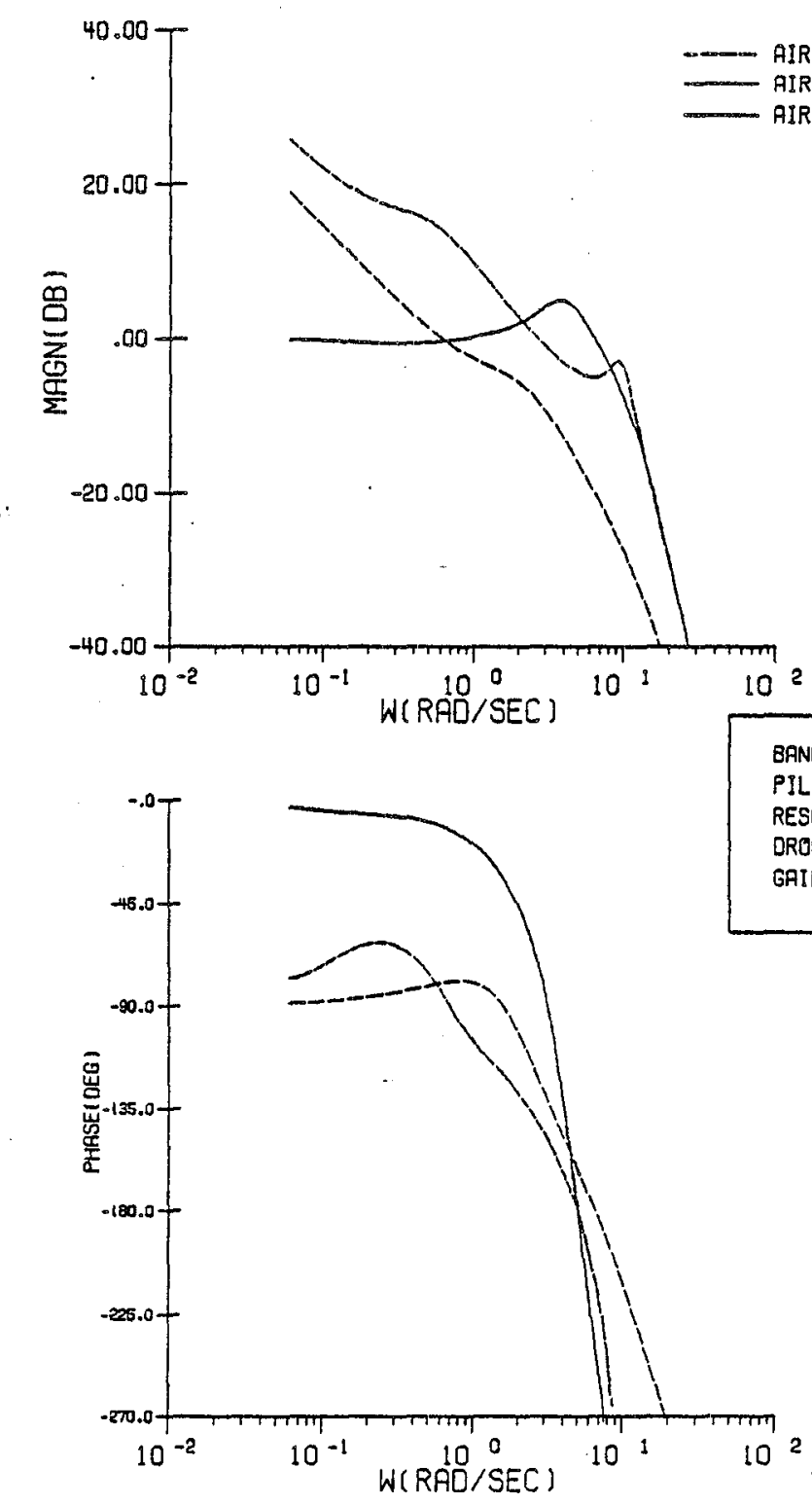

Figure C.9 Configuration $1 C /$ Corrected System Frequency Response 
CONFIGURATION 10
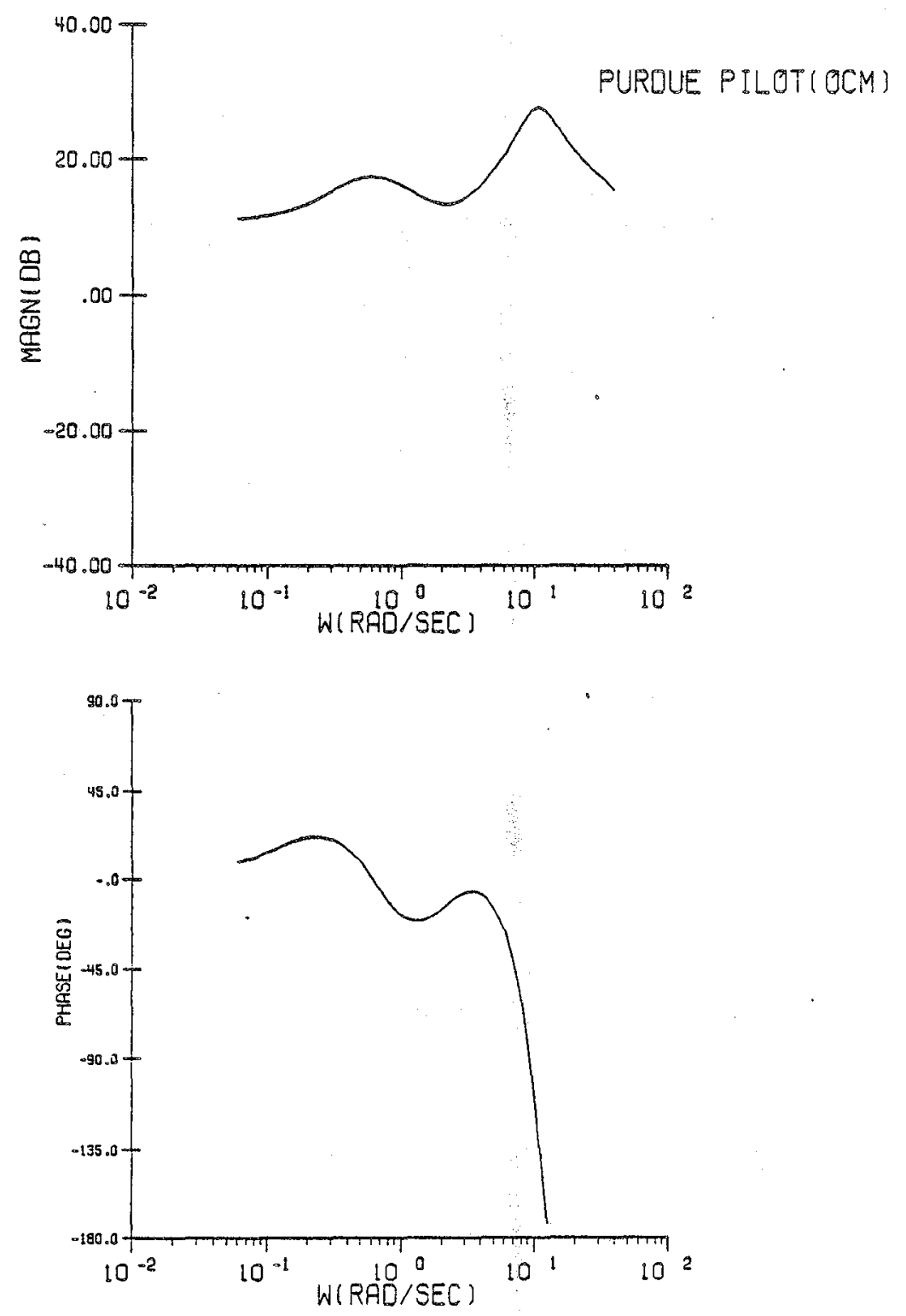

Figure C.10 Configuration 1D/Pilot Frequency Response 


\section{CONF IGURATION 10}

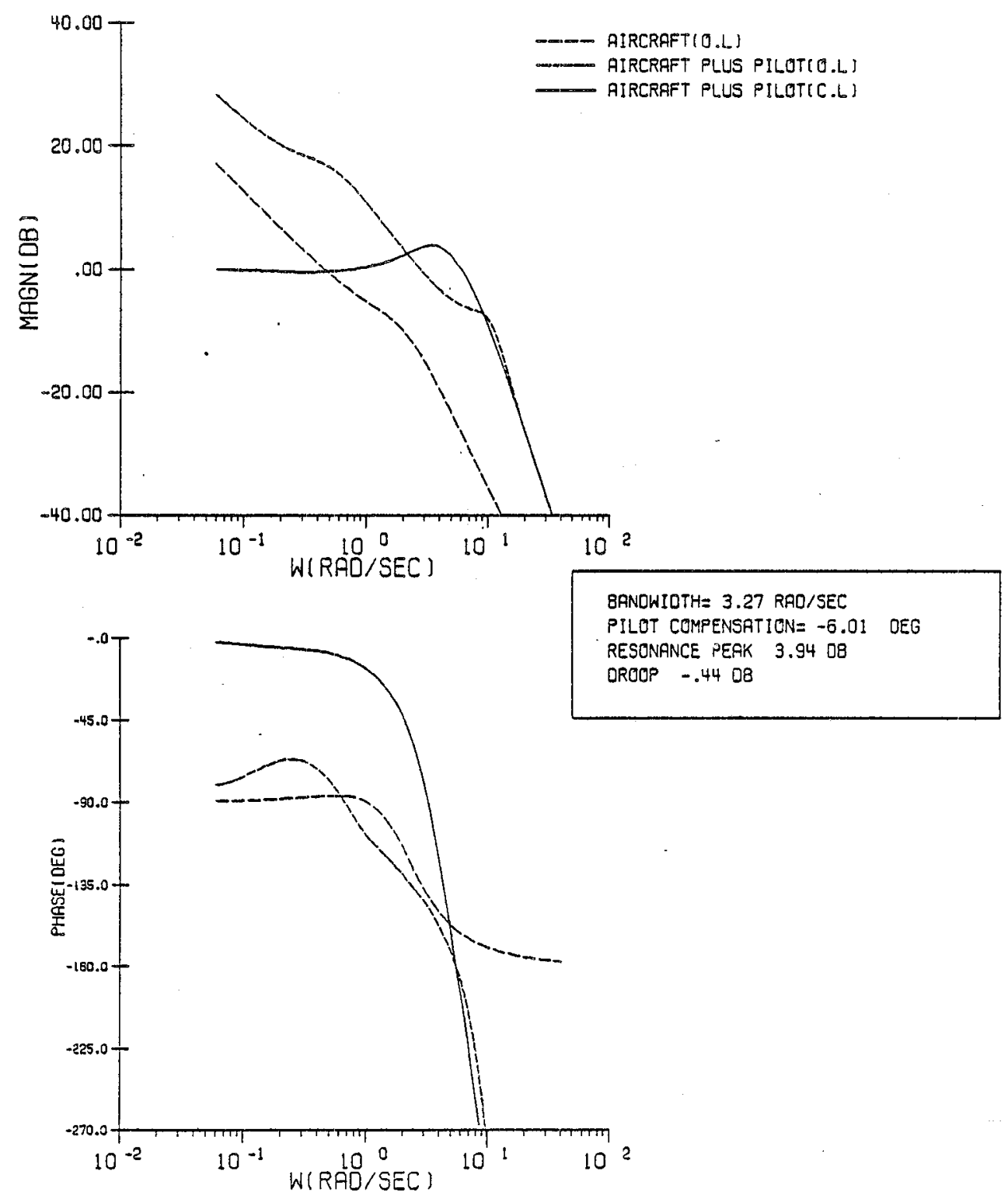

Figure C.11 Configuration 1D/System Frequency Response 
CONFIGURATION 10

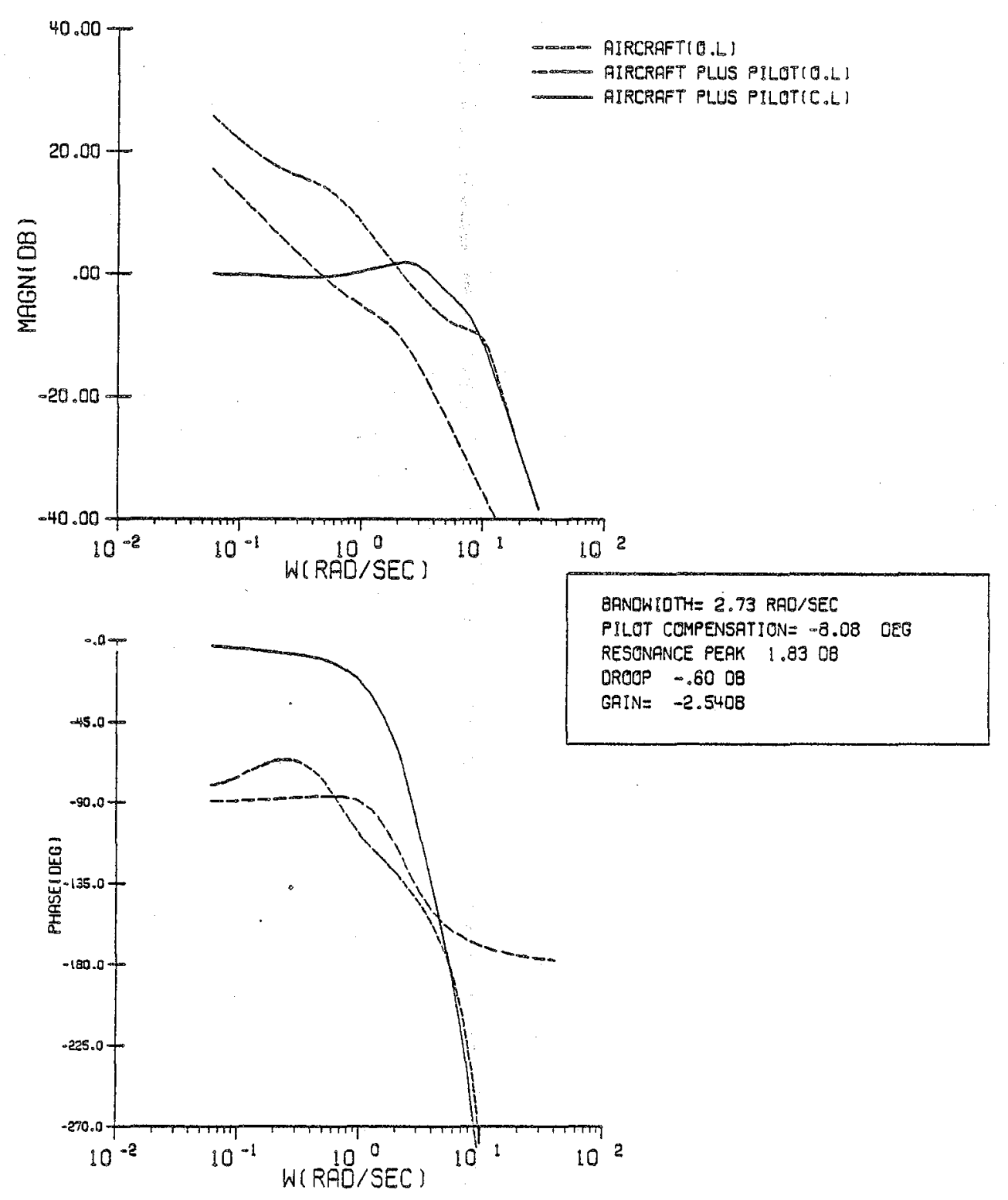

Figure C.12 Configuration 1D/Corrected System Frequency Response 


\section{CONF IGURATION $1 E$}
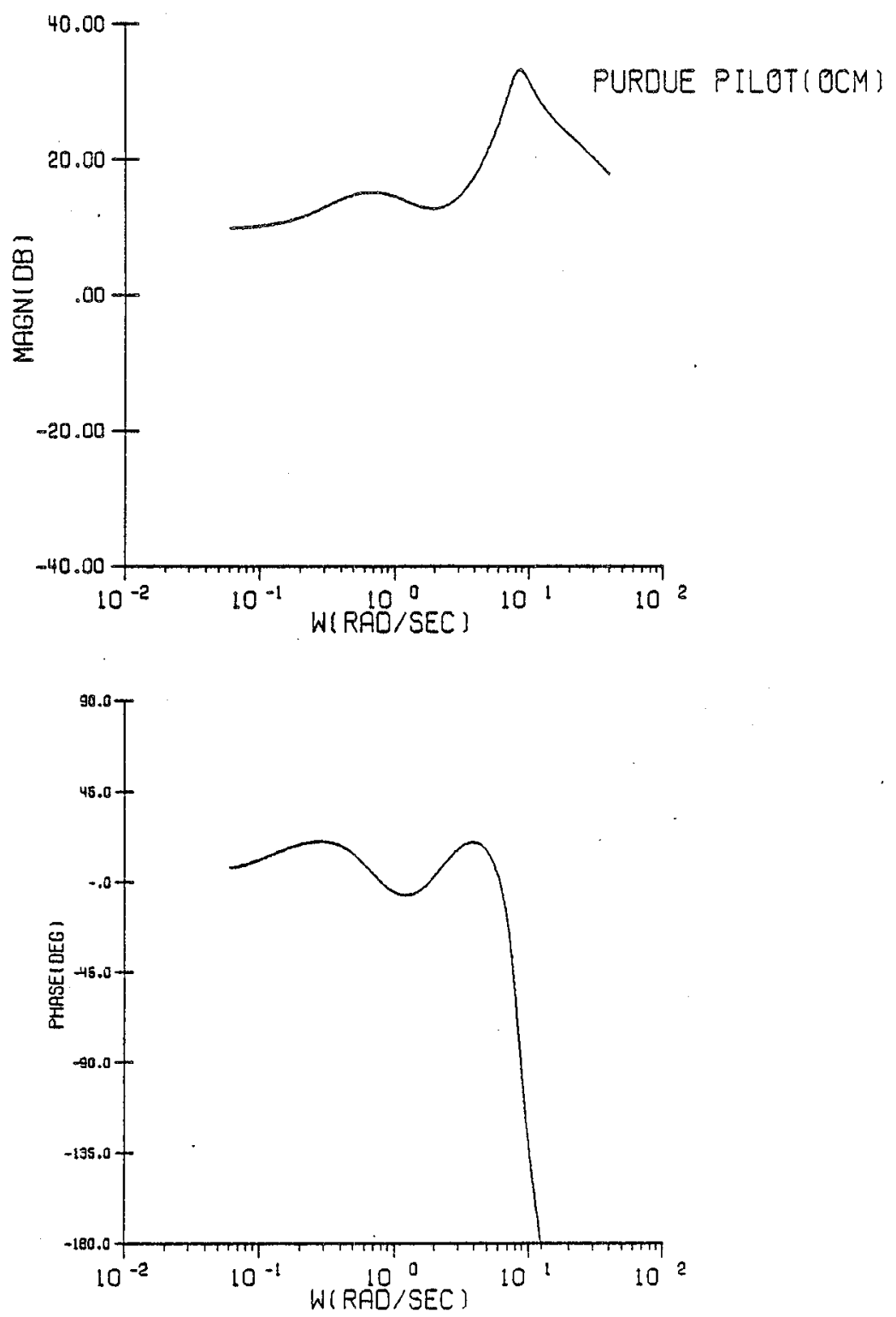

Figure C.13 Configuration 1E/Pilot Frequency Response 
CONFIGURATION IE

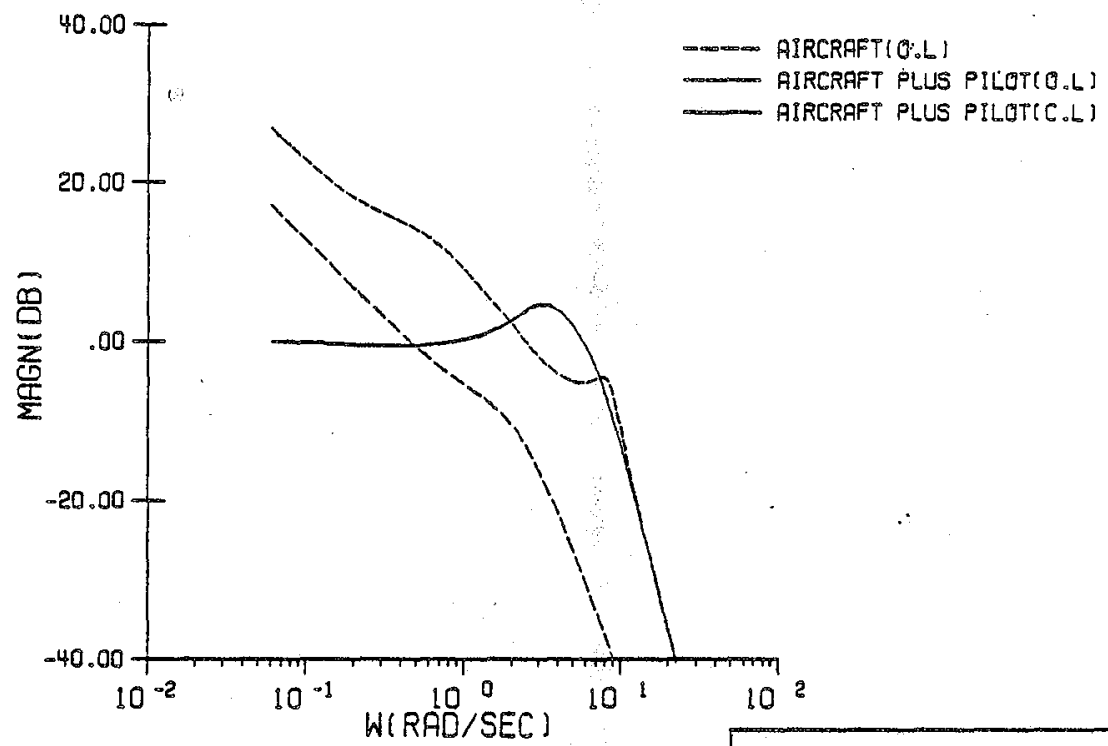

BPNOWIDTH= 2.84 RAQ/SEC

PILOT COMPENSATION= 14.74 OEG

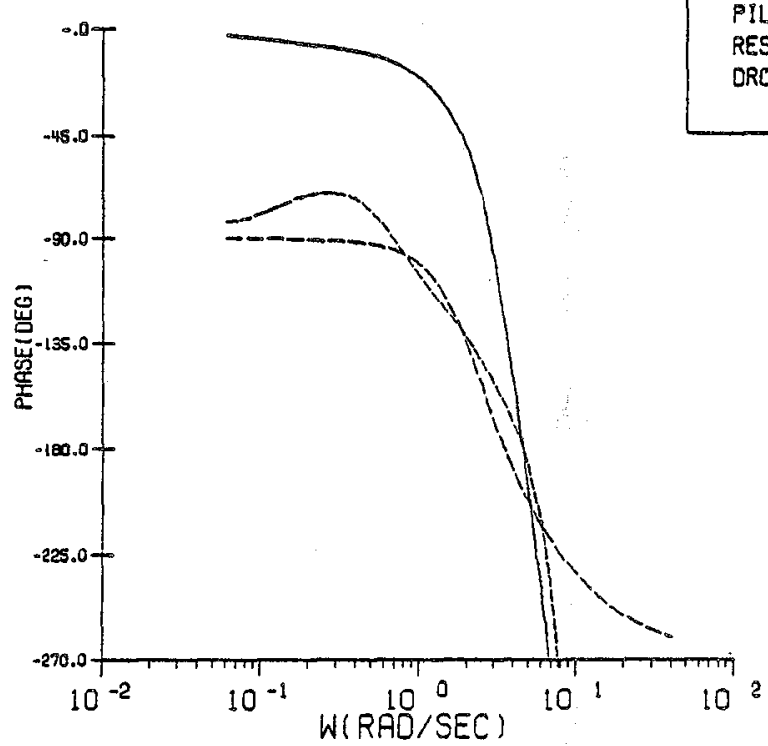

Figure C.14 Configuration $1 E /$ Sys tem Frequency Response 


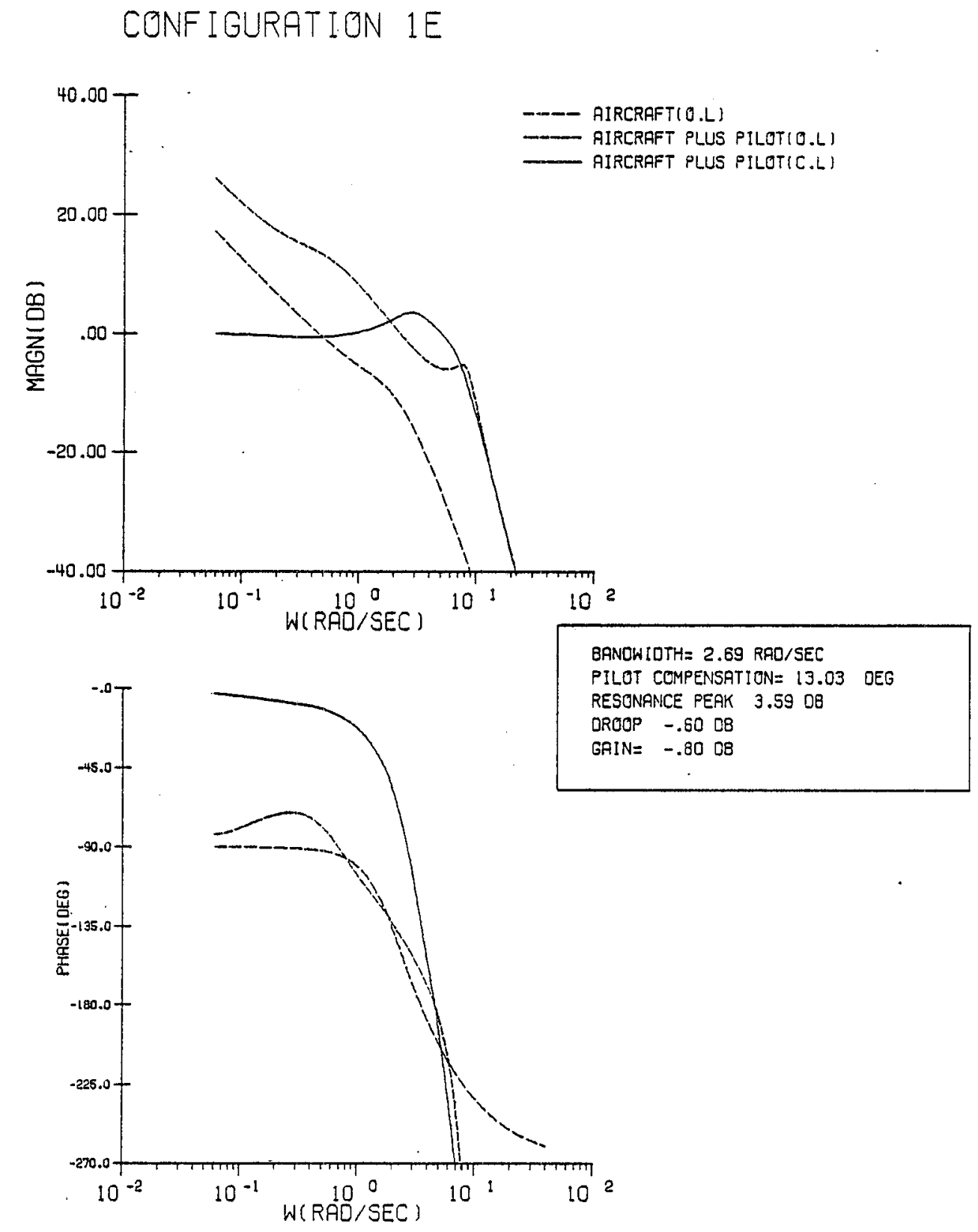

Figure C.15 Configuration $1 E /$ Corrected System Frequency Response 
CONFIGURATION IF
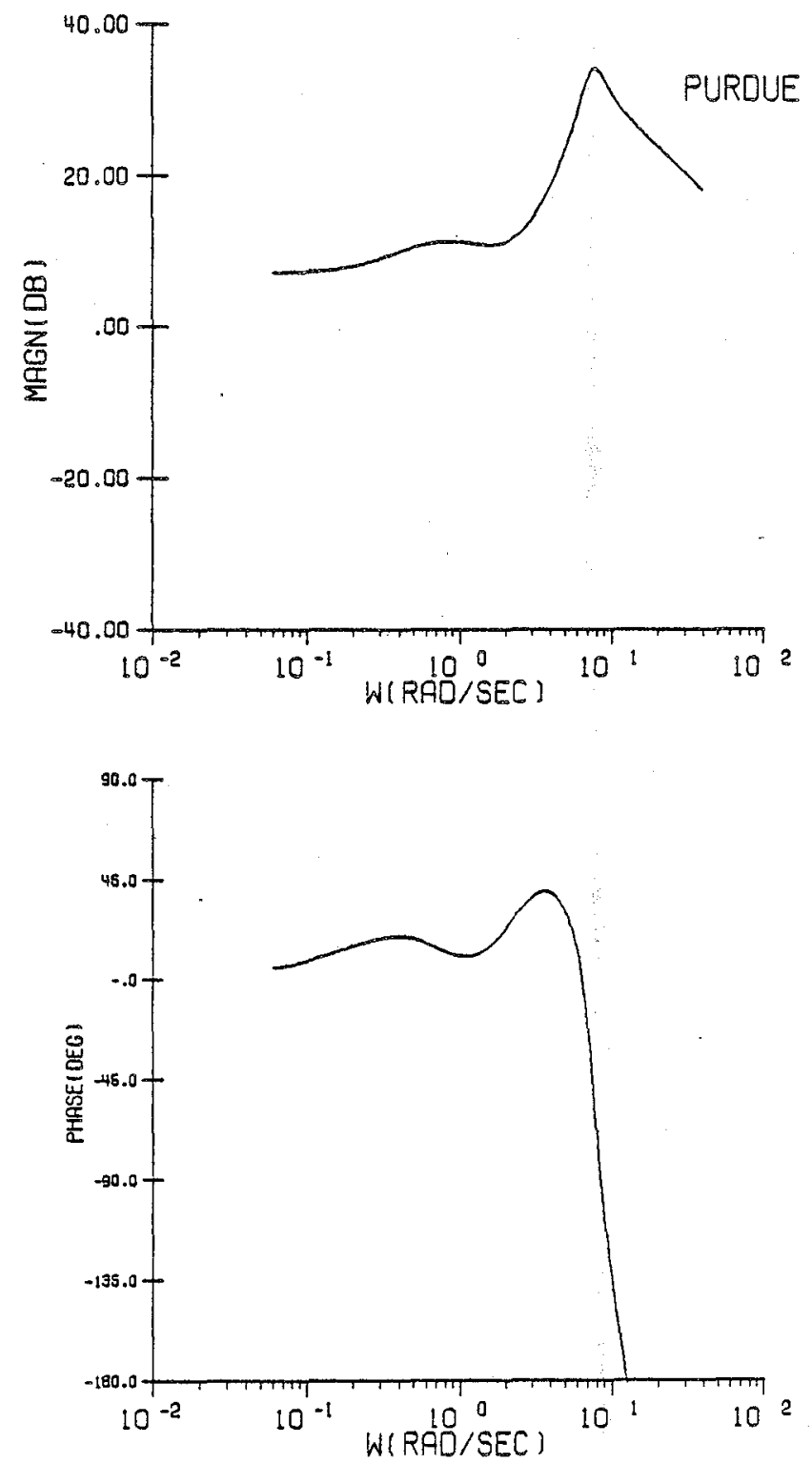

Figure C.16 Configuration 1F/Pilot Frequency Response 


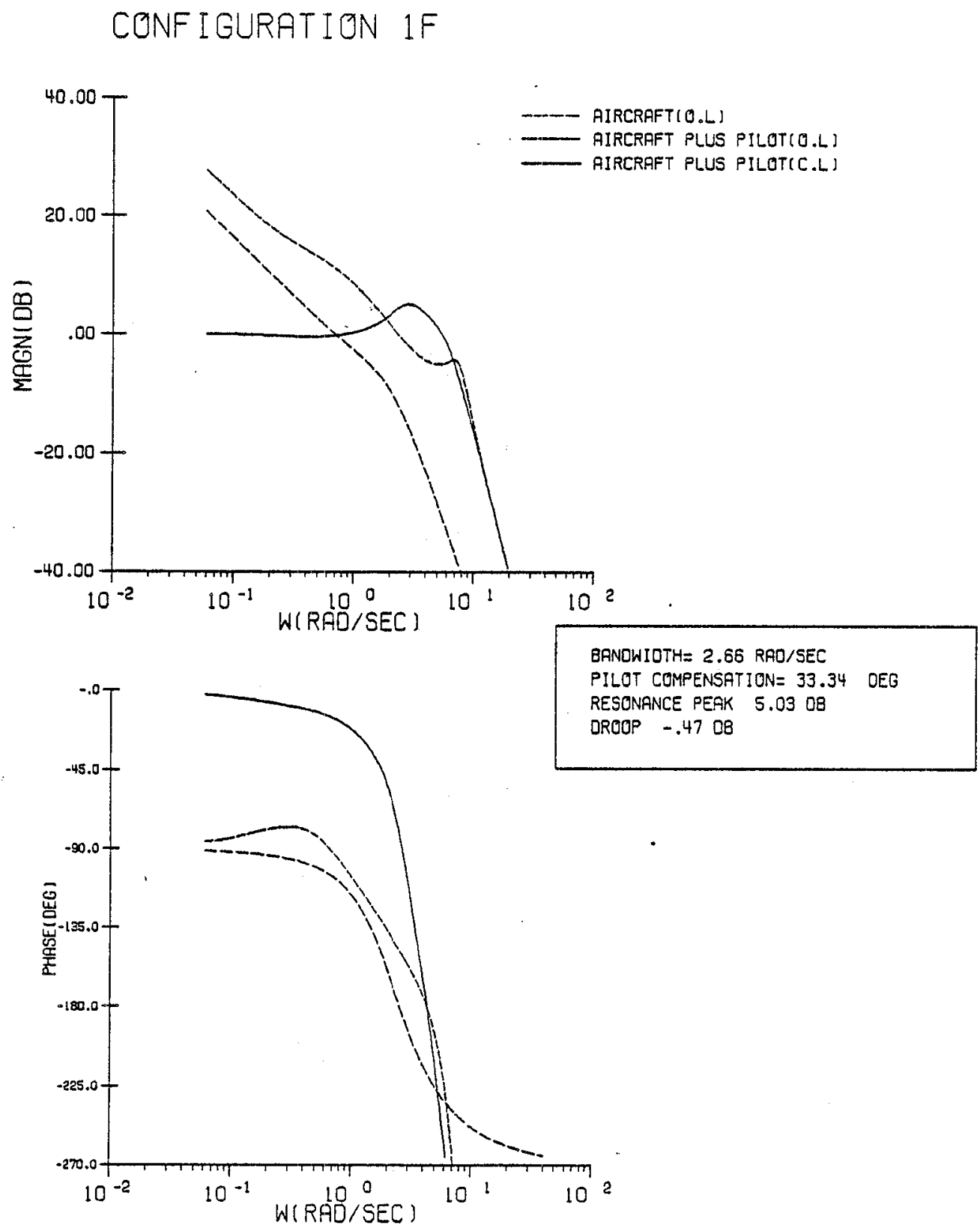

Figure $\mathrm{C} .17$ Configuration $1 \mathrm{~F} /$ Sys tem Frequency Response 
CONFIGURATION $1 \mathrm{G}$
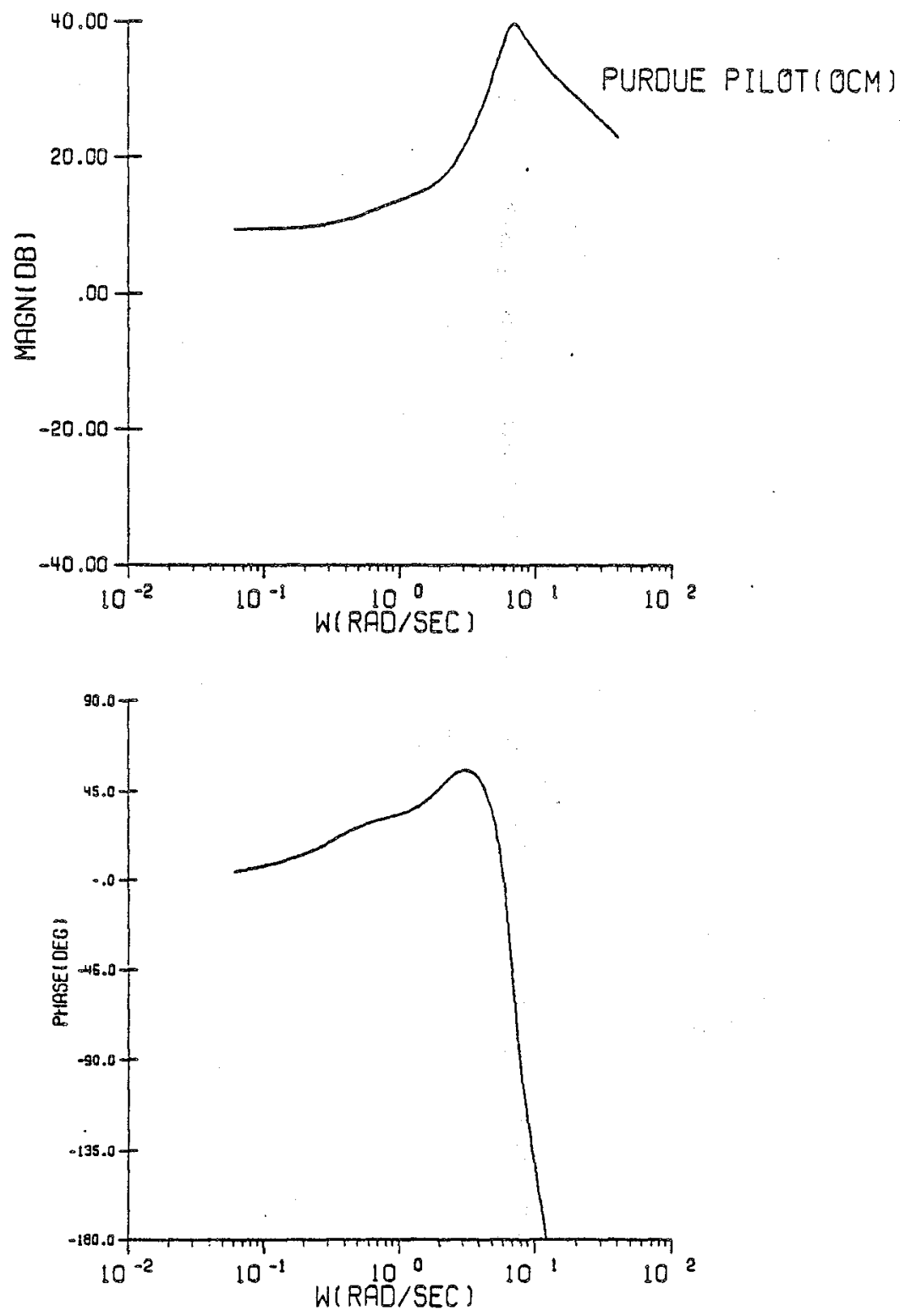

Figure $C .18$ Configuration $\mathrm{IG} / \mathrm{Pi}$ lot Frequency Response 
CONF IGURATION $1 G$

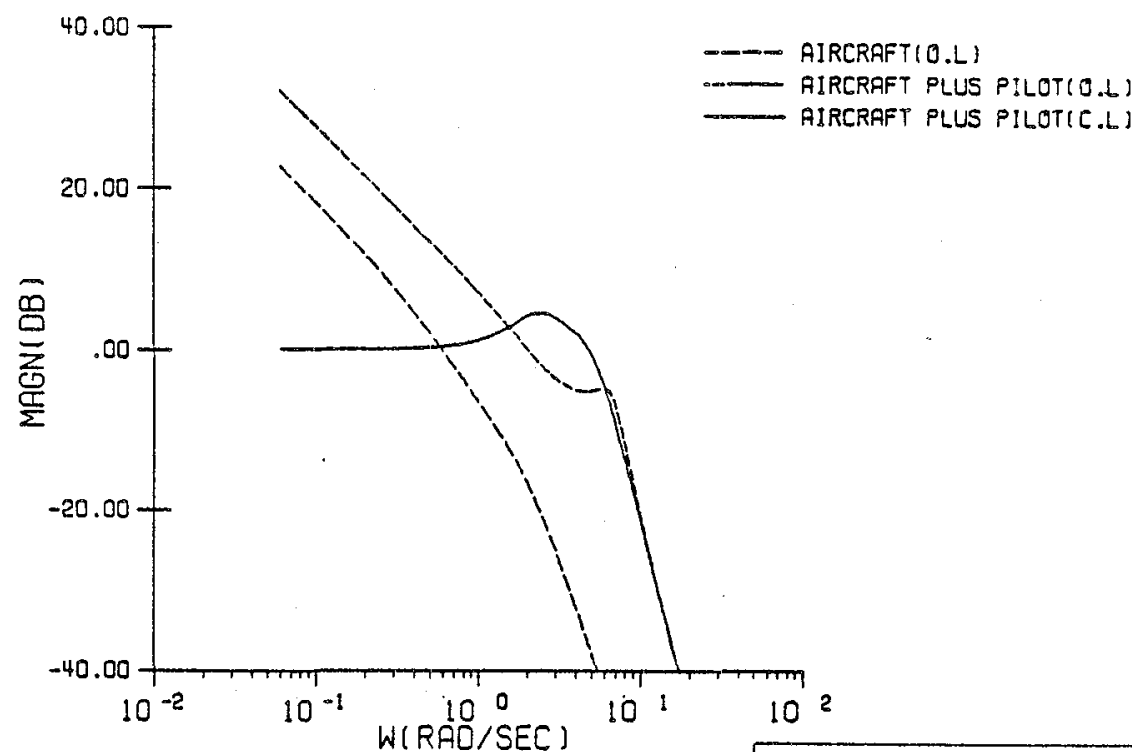

GANOWIDTH $=2.31 \mathrm{RAO} / \mathrm{SEC}$

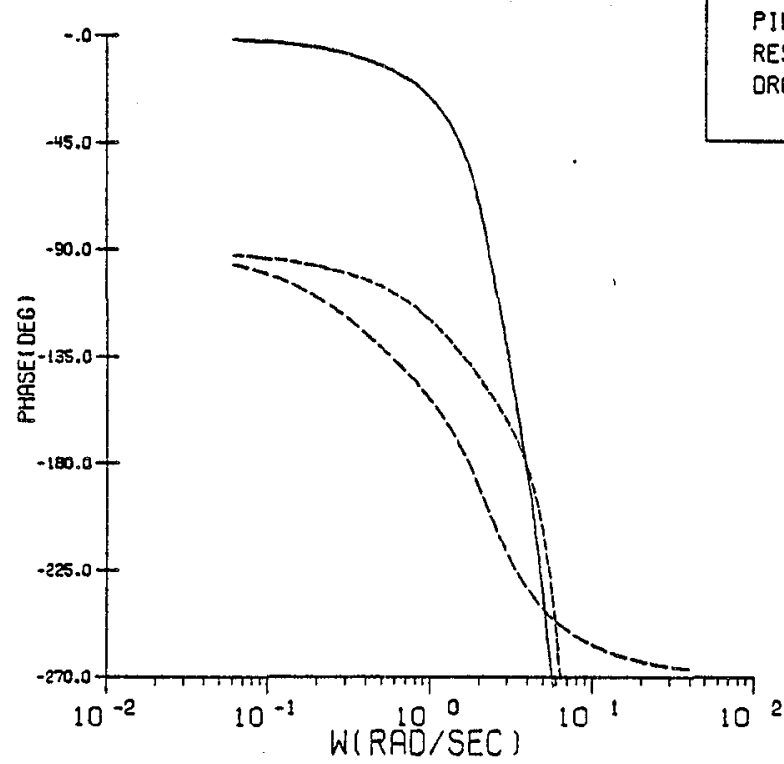

Figure C.19 Configuration $1 G /$ System Frequency Response 
CONF IGURATION $2 A$
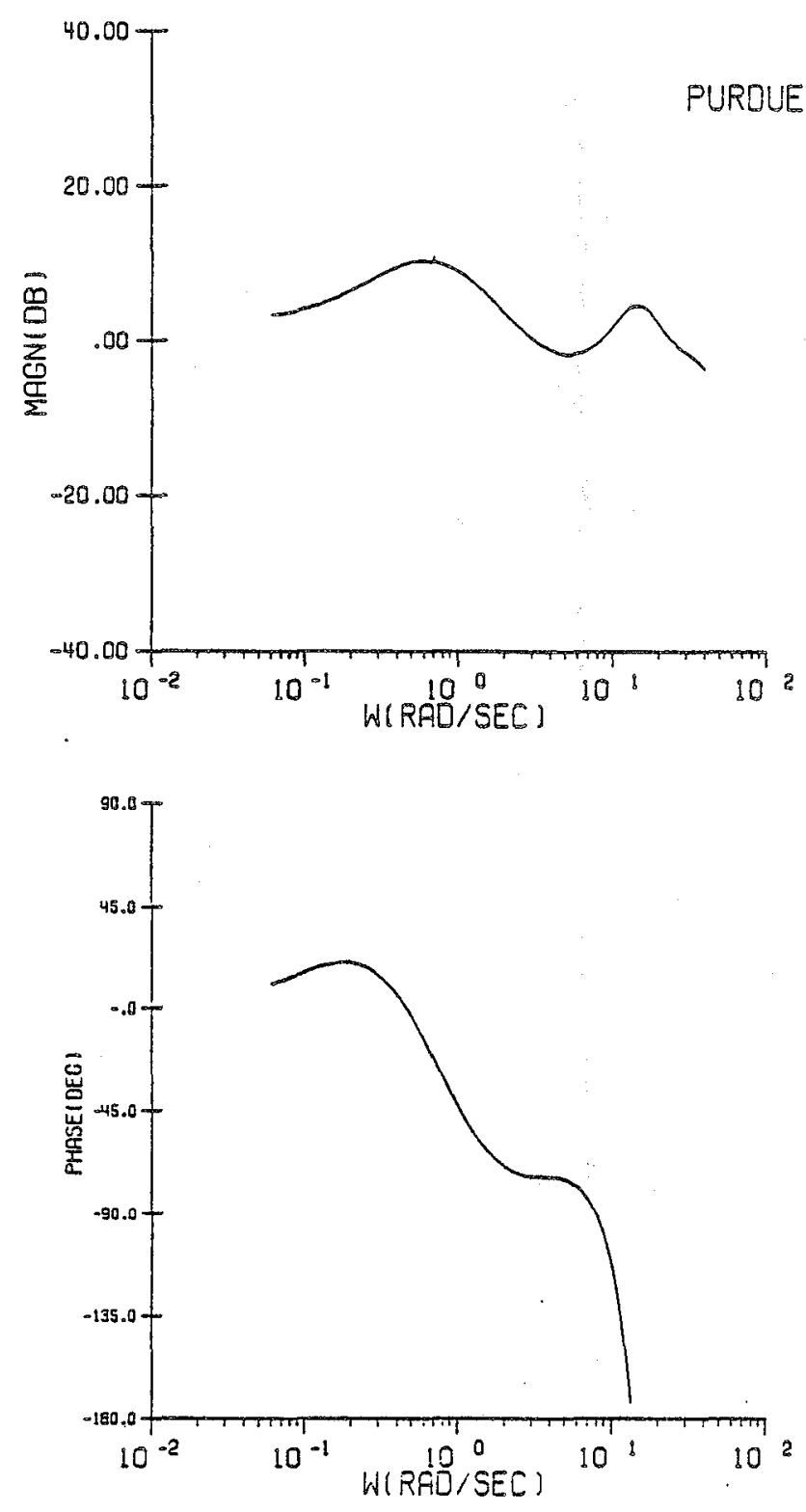

Figure C.20 Configuration $2 A / P$ ilot Frequency Response 
CONFIGURATION $2 A$

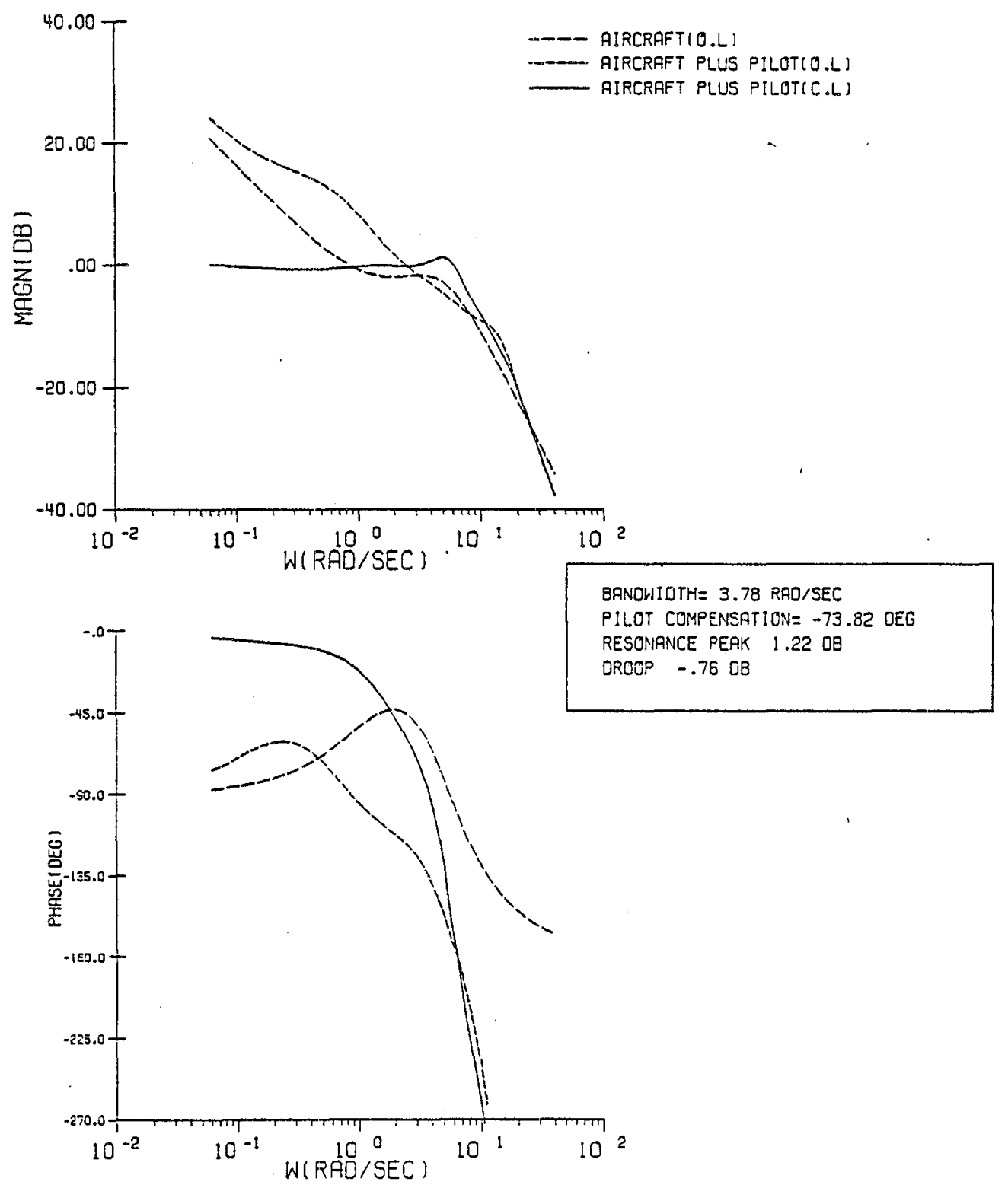

Figure C.21 Configuration 2A/Sys tem Frequency Response 
CONF IGURATION 2A

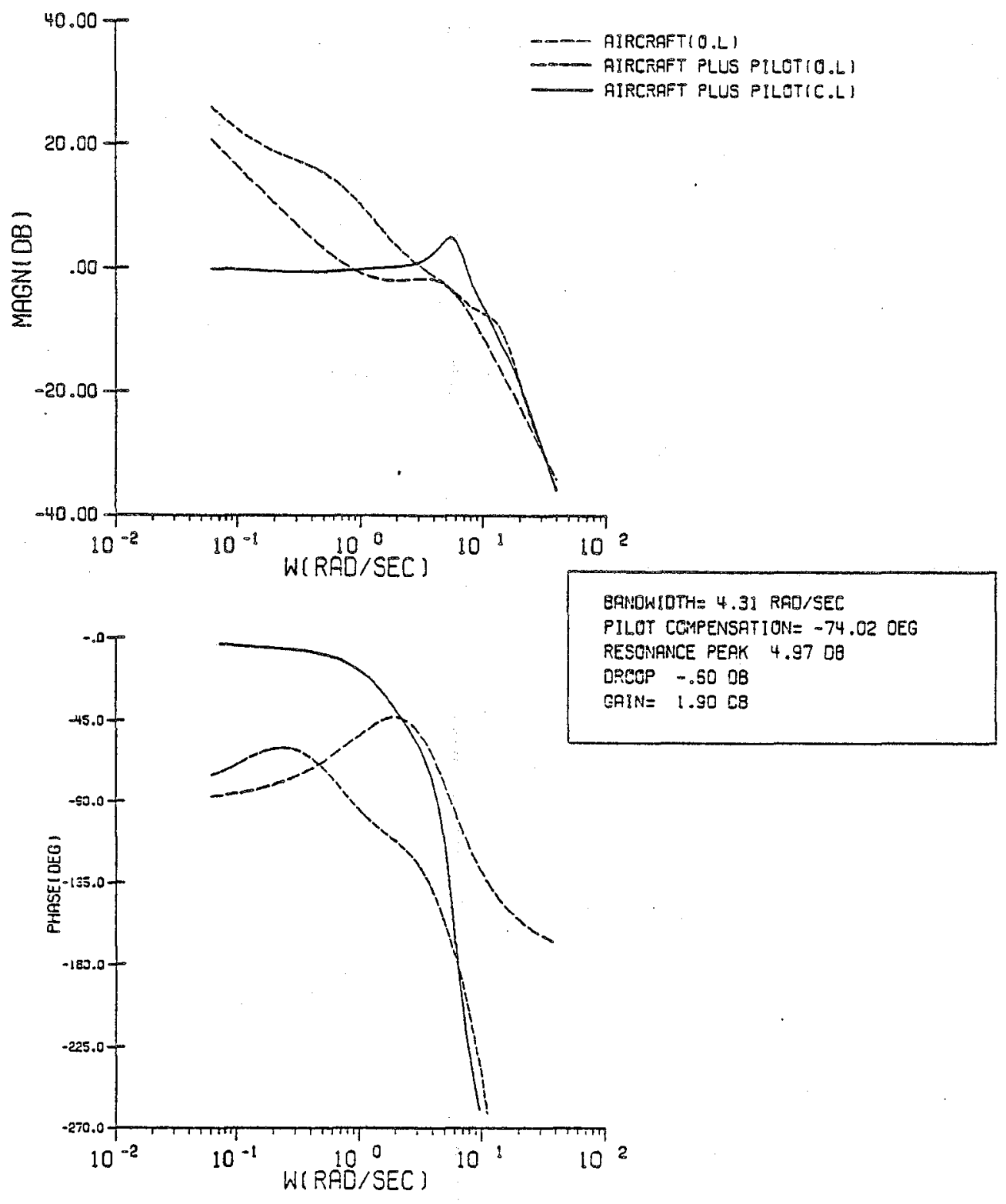

Figure C.22 Configuration 2A/Corrected System Frequency Response 
CONF IGURATION $2 B$
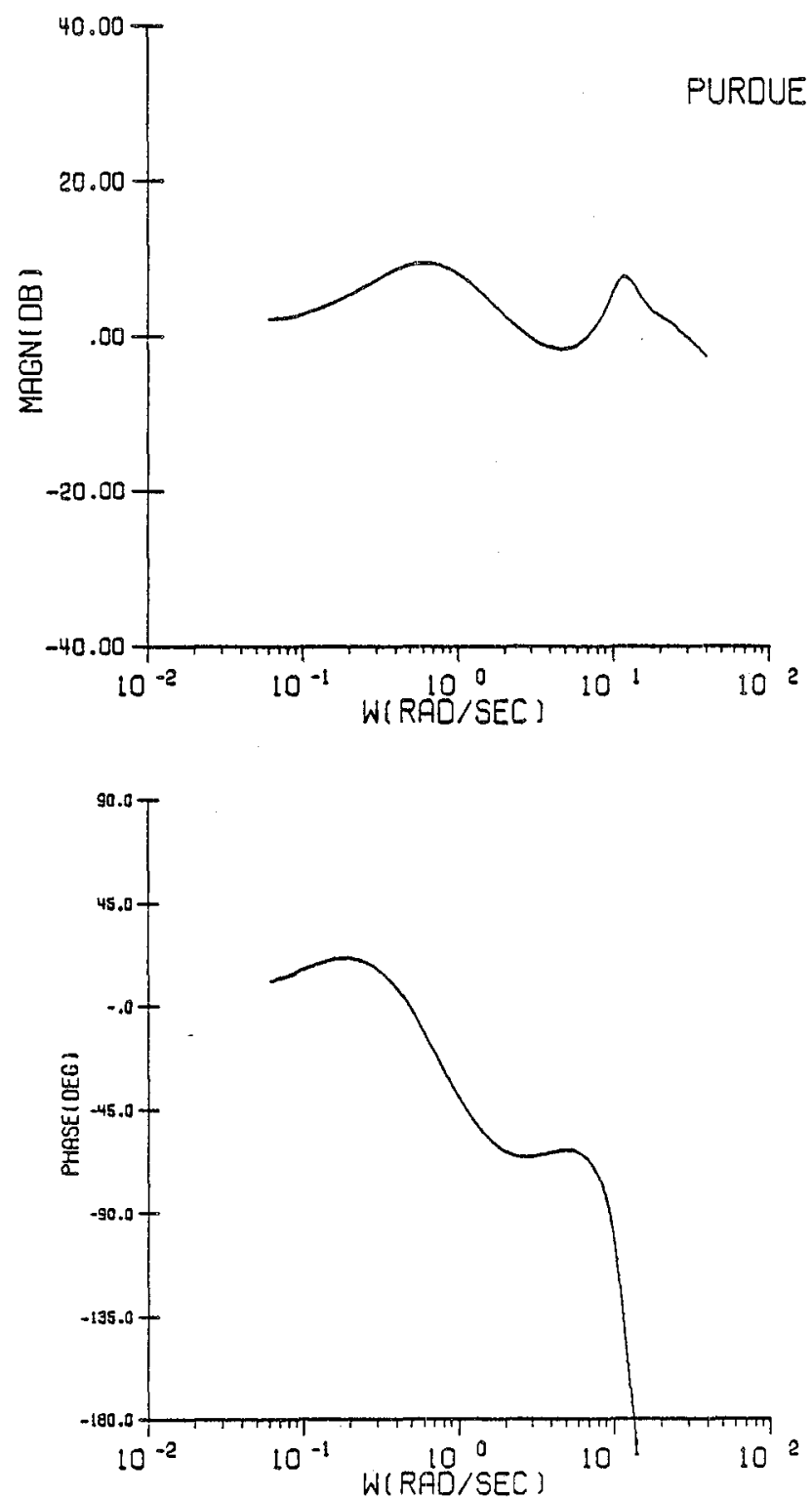

Figure C.23 Configuration 2B/Pilot Frequency Response 
CONFIGURATION 28

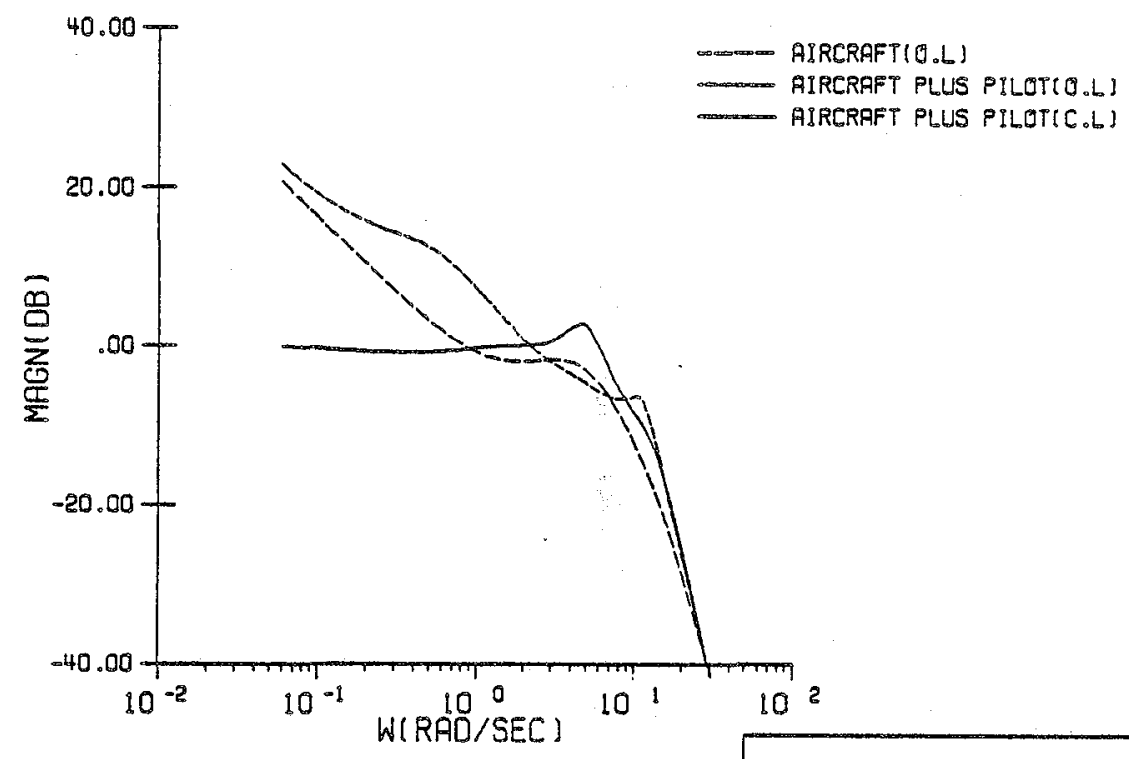

BANOWIOTH $=3.32$ RRO/SEC

PILOT COMPENSATION $=-64.72$ OEG

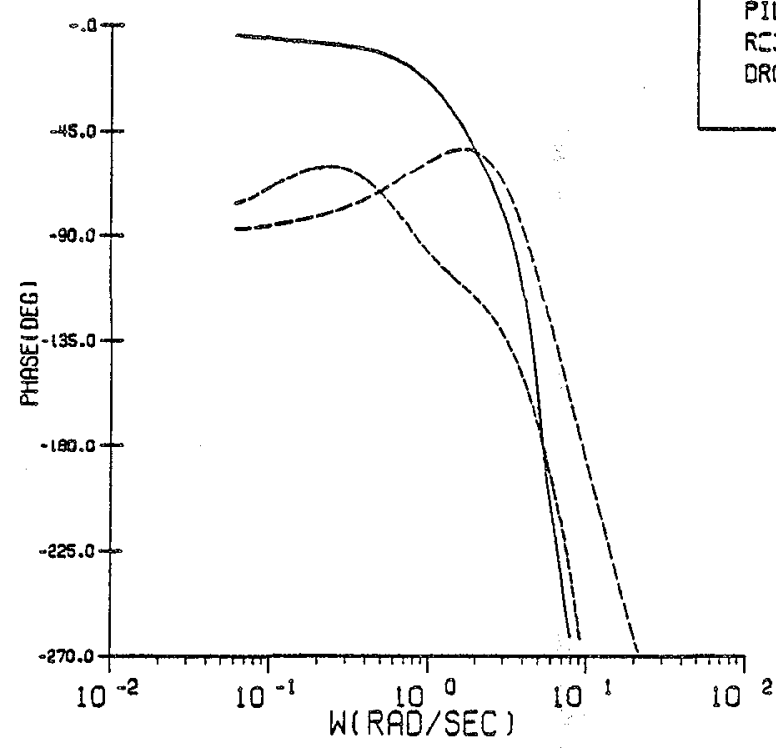

Figure C.24 Configuration 2B/Sys tem Frequency Response 


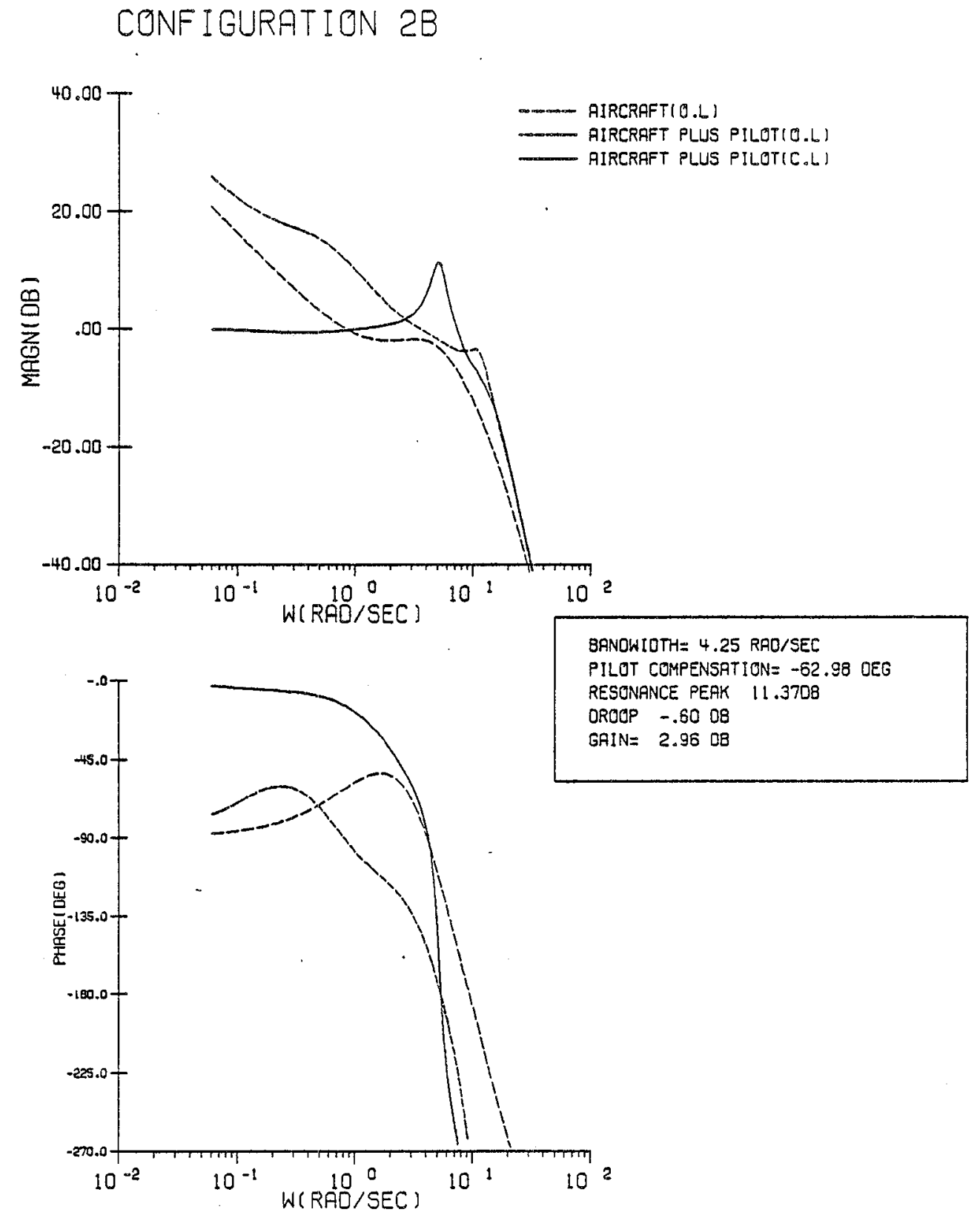

Figure C.25 Configuration $2 B /$ Corrected System Frequency Response 
CONF IGURATION $2 C$
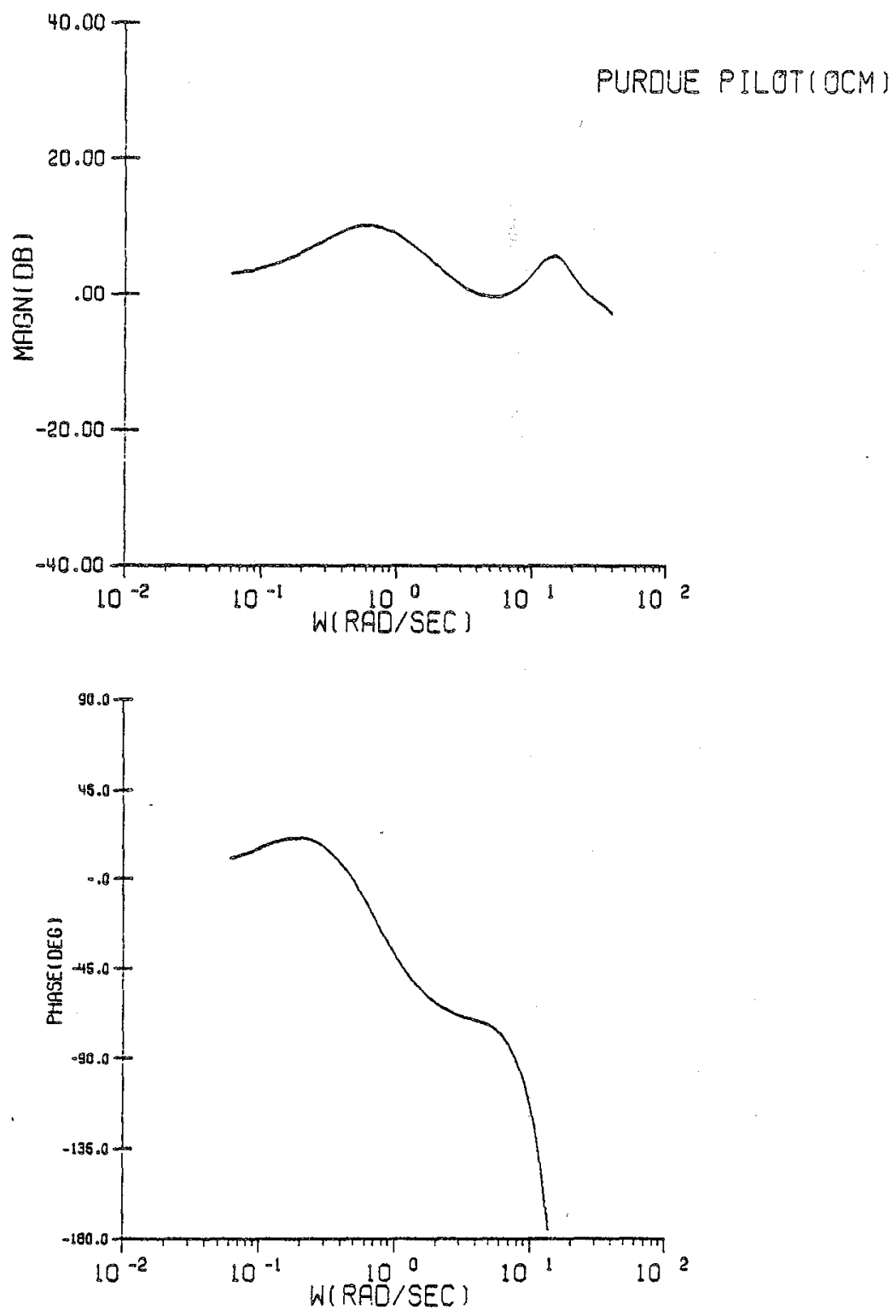

Figure $\mathrm{C} .26$ Configuration $2 \mathrm{C} / \mathrm{Pi}$ lot Frequency Response 
CONFIGURATION 2C

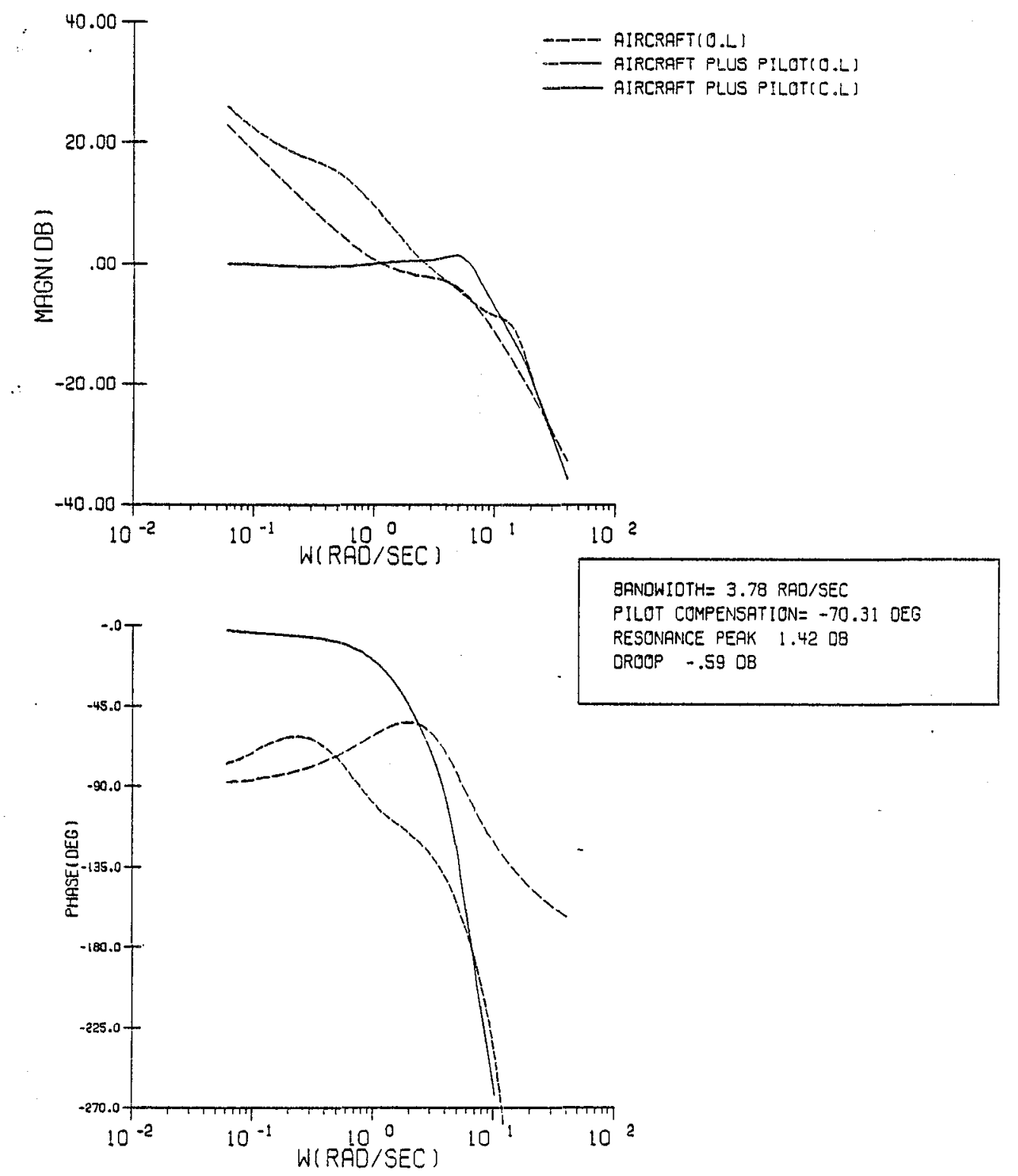

Figure C.27 Configuration 2C/Sys tem Frequency Response 


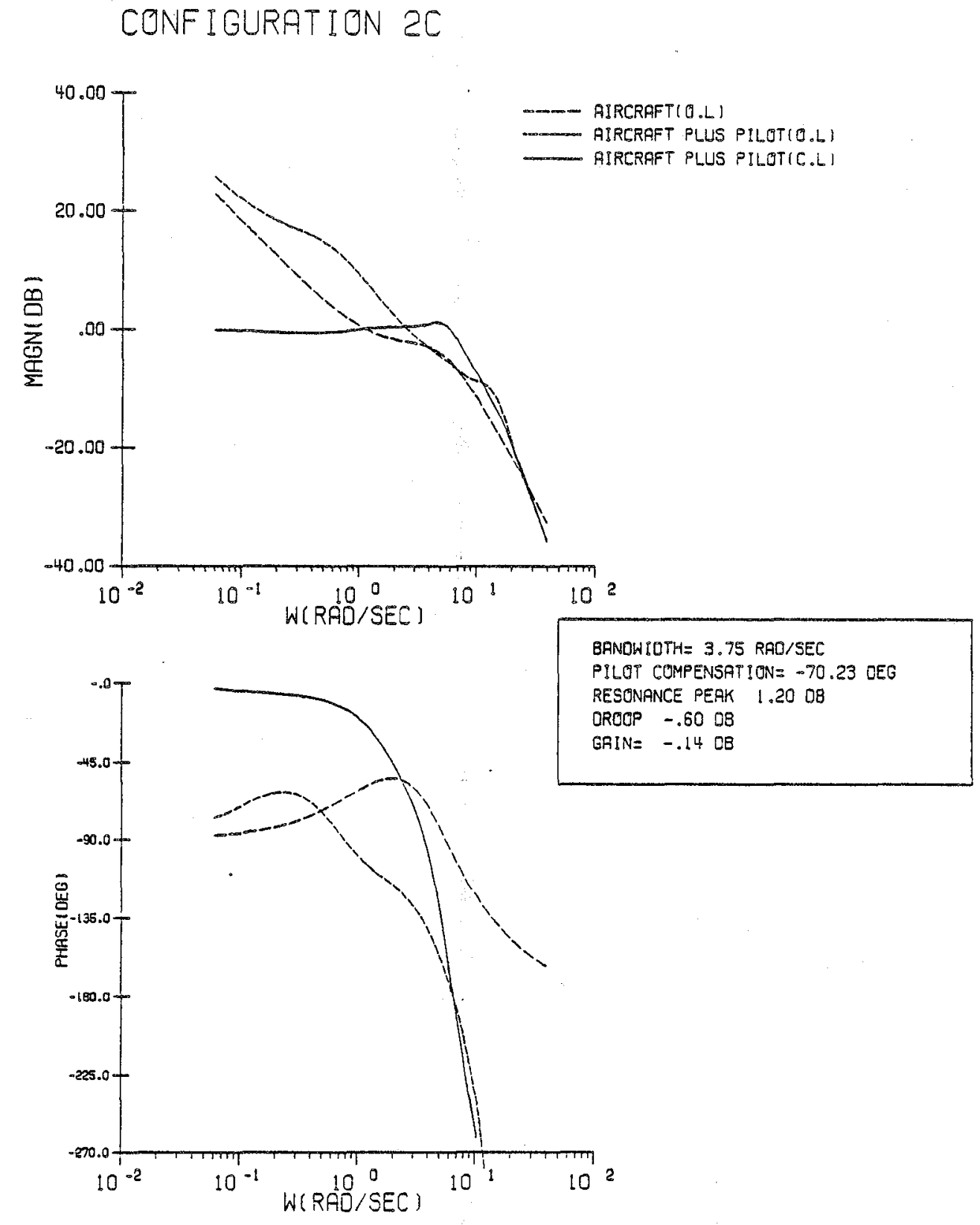

Figure C.28 Configuration $2 \mathrm{C} /$ Corrected System Frequency Response 
CONF IGURATION 20
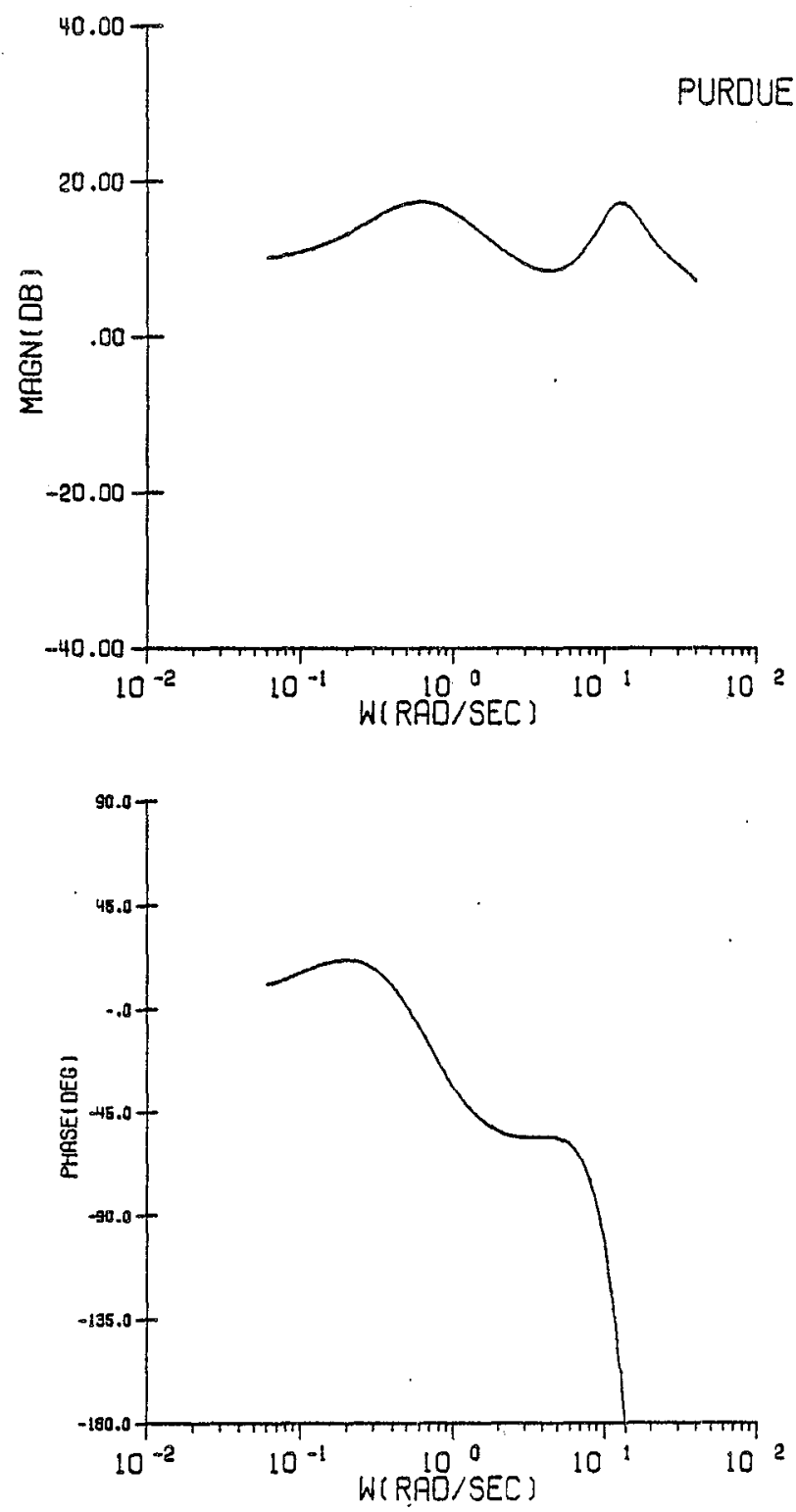

Figure C.29 Configuration 2D/Pilot Frequency Response 
CONFIGURATION 20

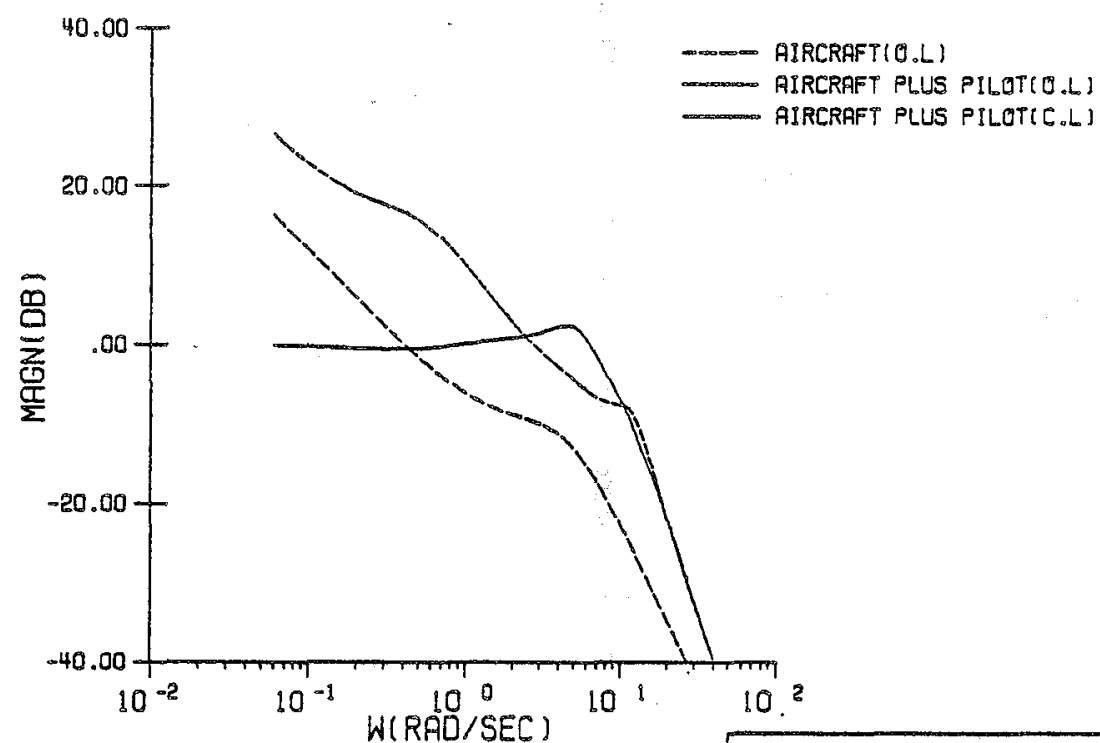

BRNDWIDTH $=3.68$ RAD/SEC

PILOT COMPENSATION $=-55.61$ LEG

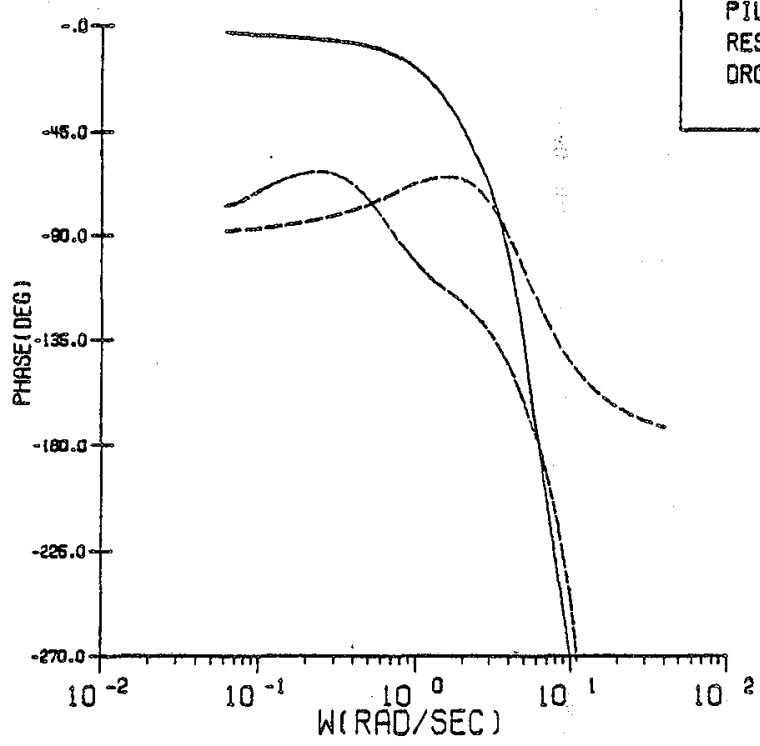

Figure C.30 Configuration 2D/Sys tem Frequency Response 
CONFIGURATION 20

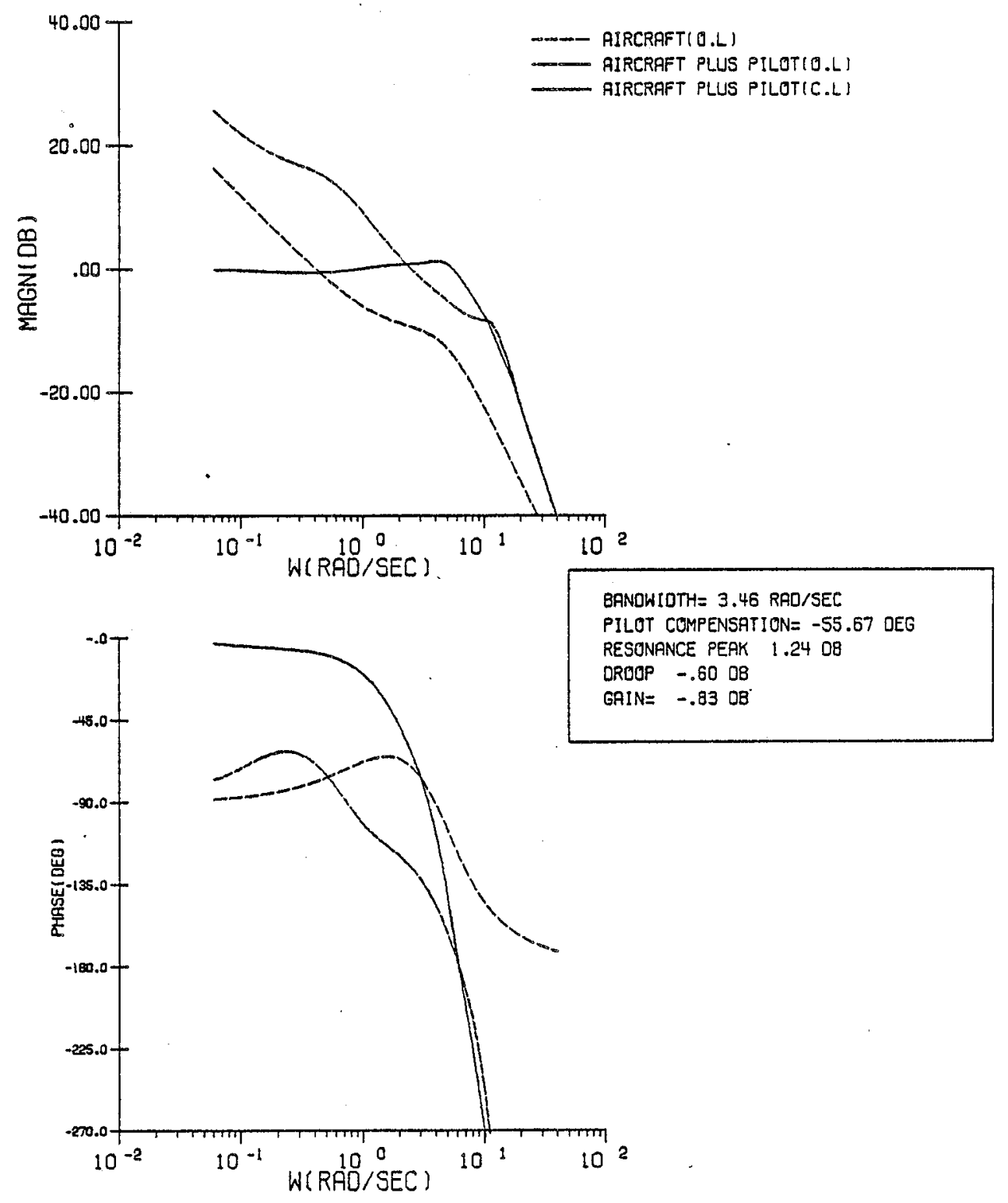

Figure C.31 Configuration 2D/Corrected System Frequency Response 
CONFIGURATION $2 E$
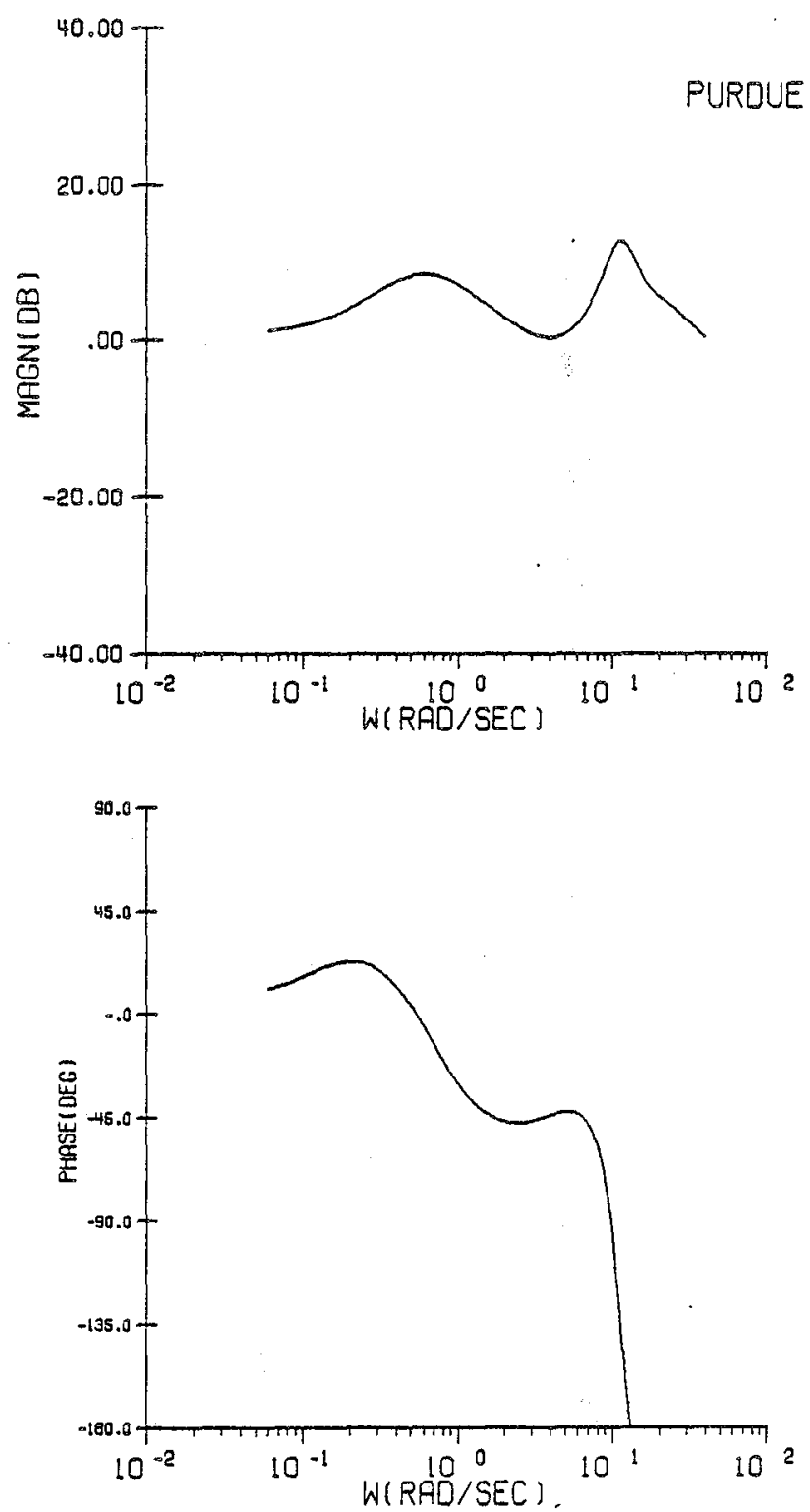

Figure C.32 Configuration 2E/Pilot Frequency Response 
CONFIGURATION ZE

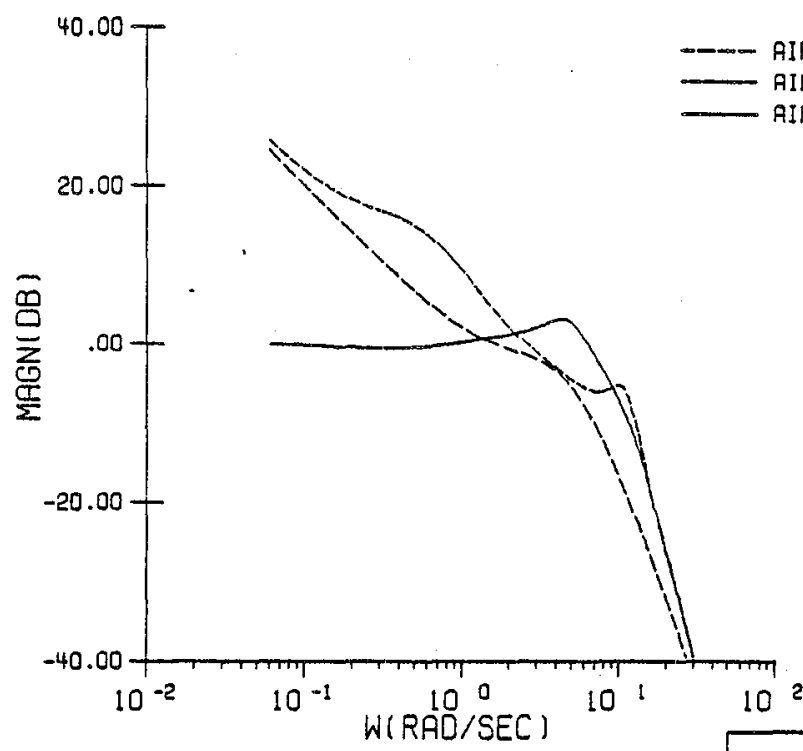

BANDWIOTH $=3.37$ RAD/SEC

PILOT COMPENSATION $=-45.69$ DEG

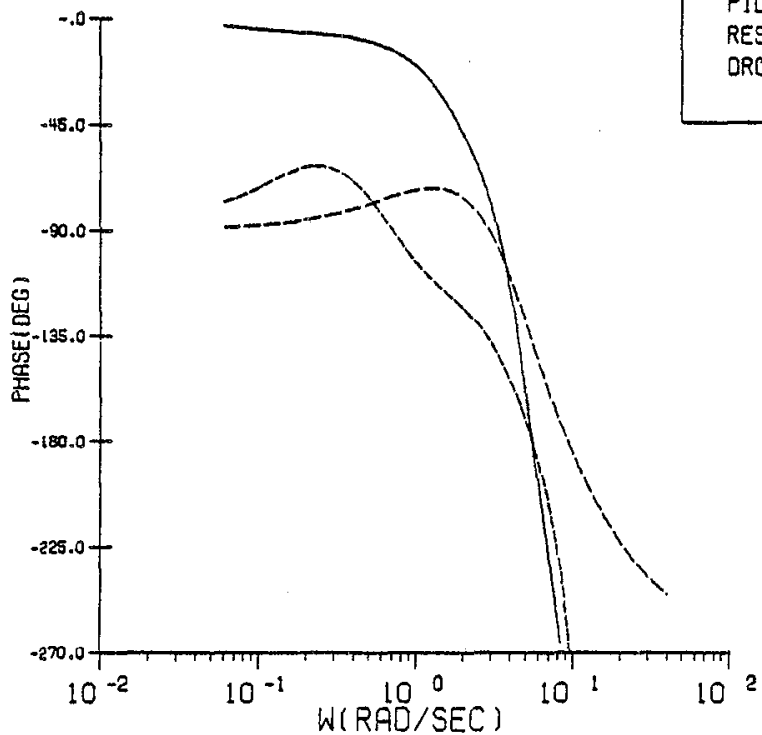

STANCE PEAK 3.21 DB

DROOP $-.60 \mathrm{OB}$

Figure C.33 Configuration 2E/System Frequency Response 
CONFIGURATION ZE
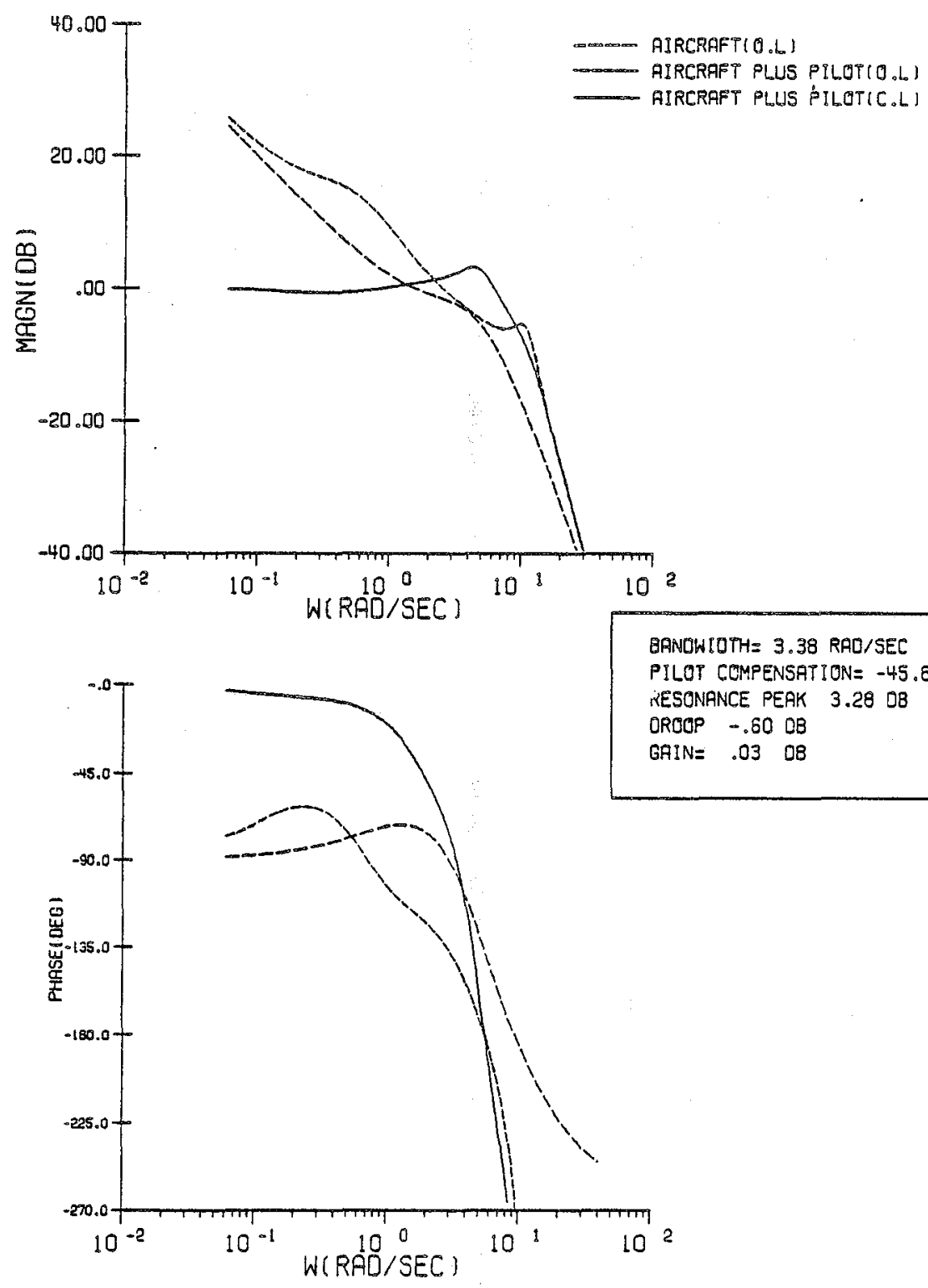

Figure C.34 Configuration 2E/Corrected Sys tem Frequency Response 
CONF IGURATION 2F
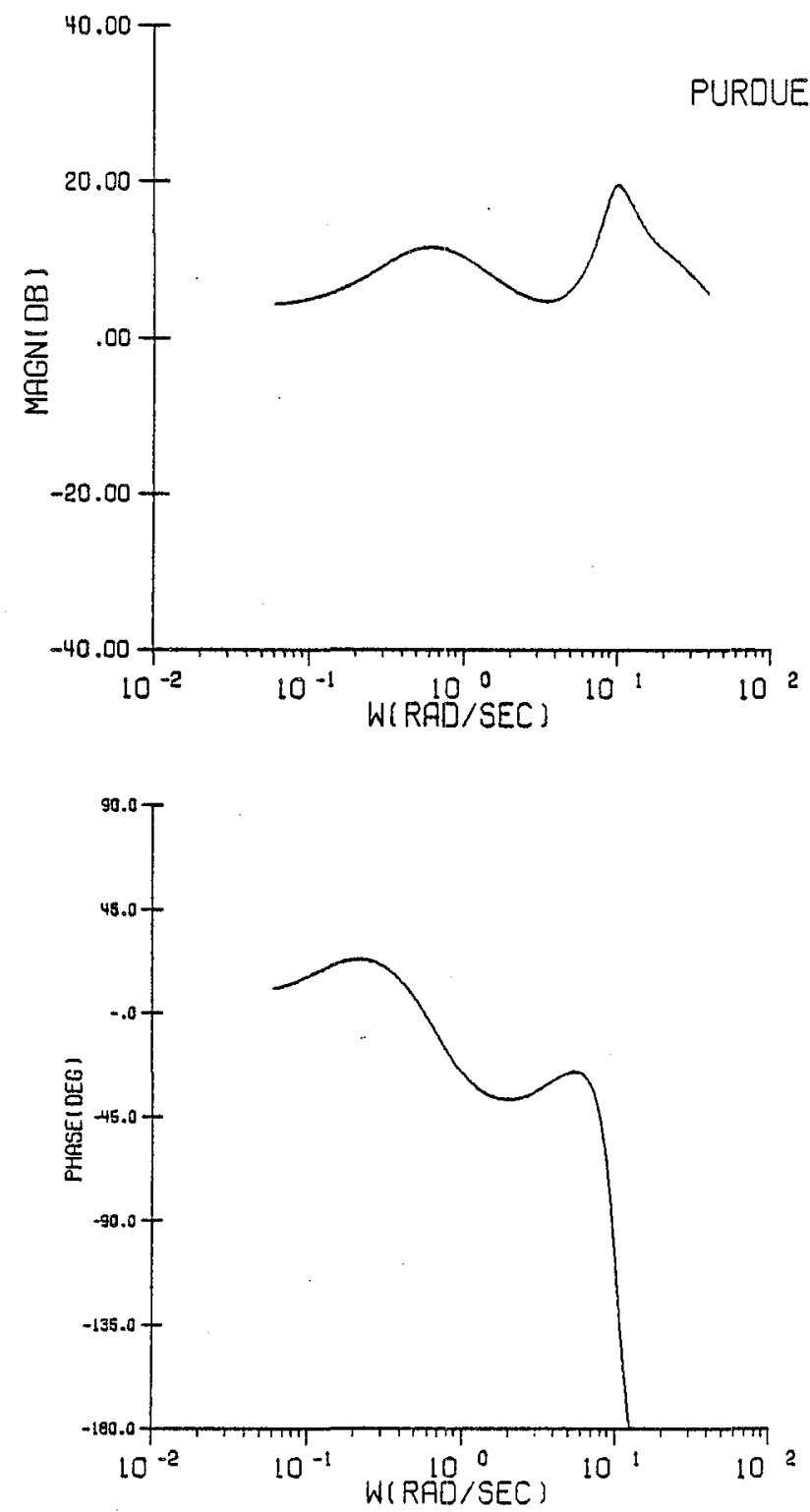

Figure C.35 Configuration 2F/Pilot Frequency Response 


\section{CONF IGURATION 2F}

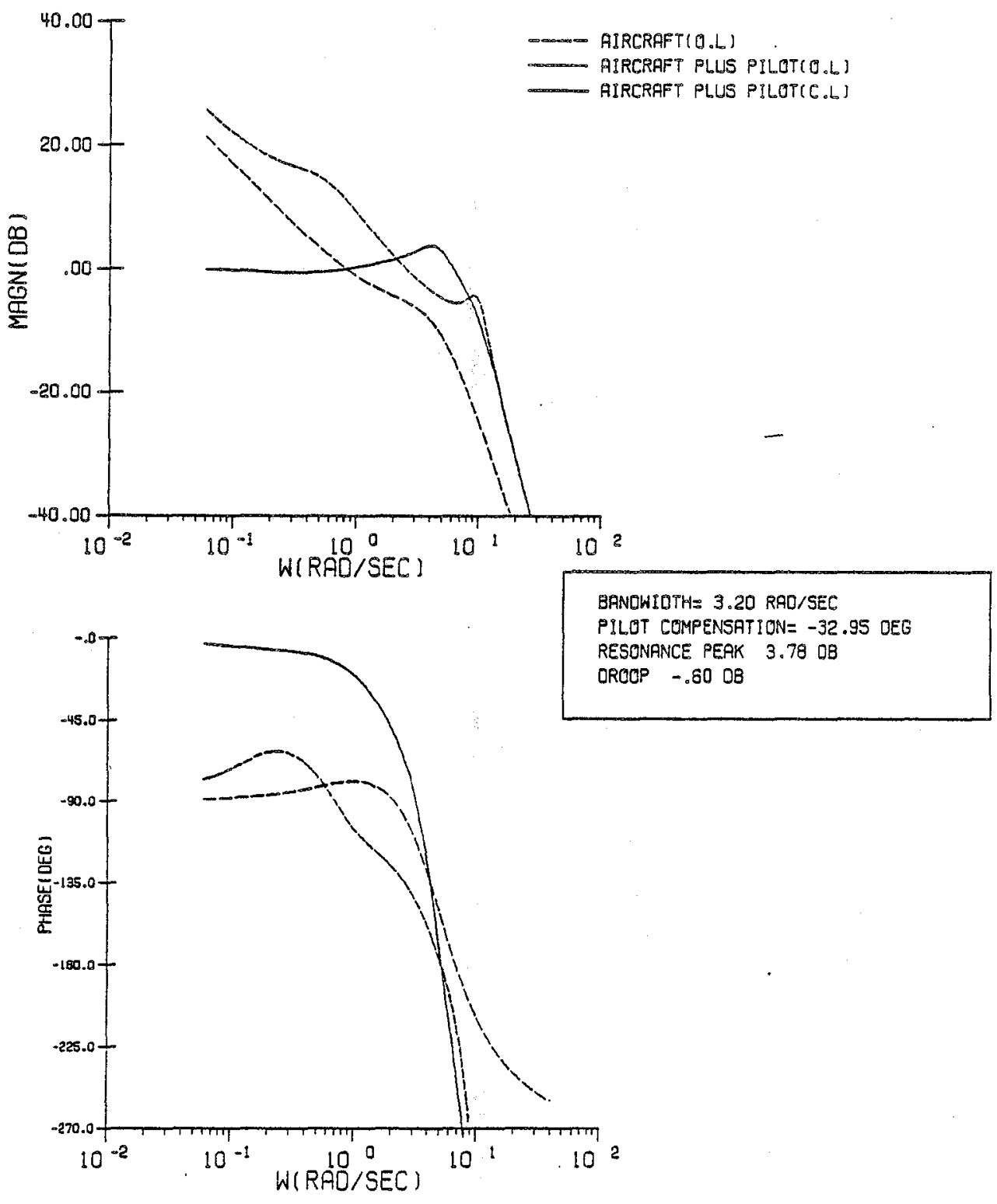

Figure C.36 Configuration $2 F /$ System Frequency Response 
CONFIGURATION $2 F$

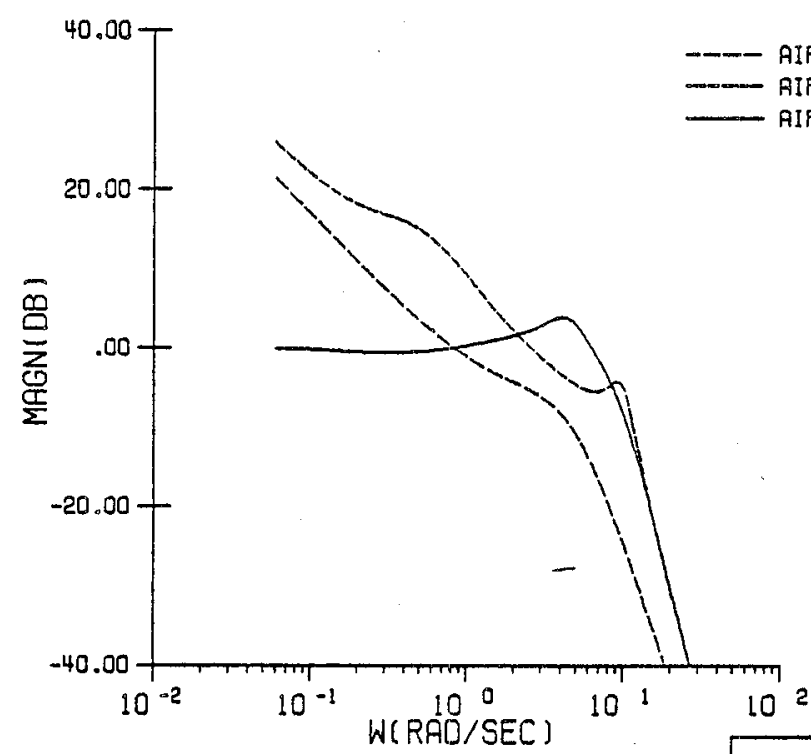

BANDWIDTH $=3.22$ RAD/SEC PILOT COMPENSATION $=-32.87$ OEG

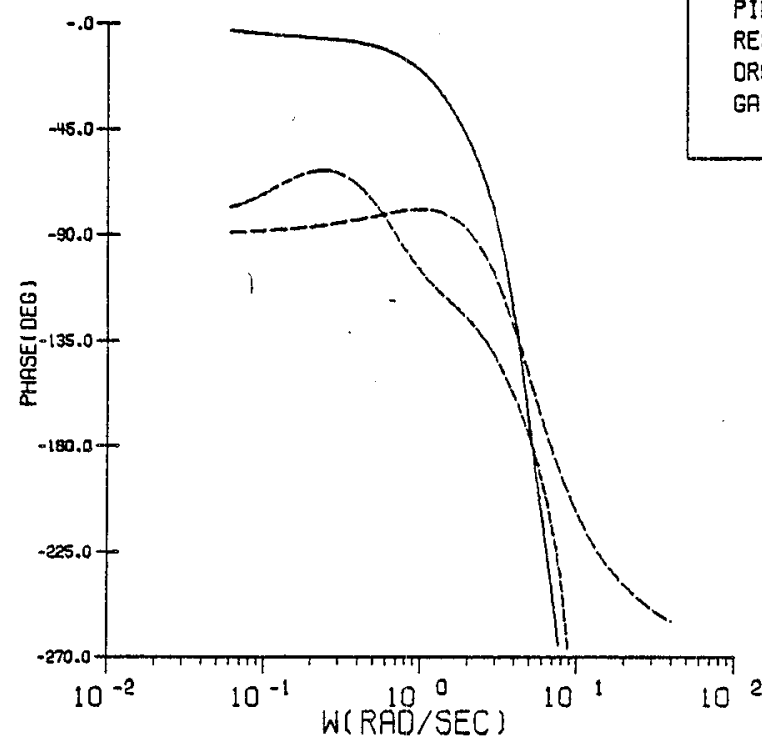

Figure C.37 Configuration 2F/Corrected System Frequency Response 


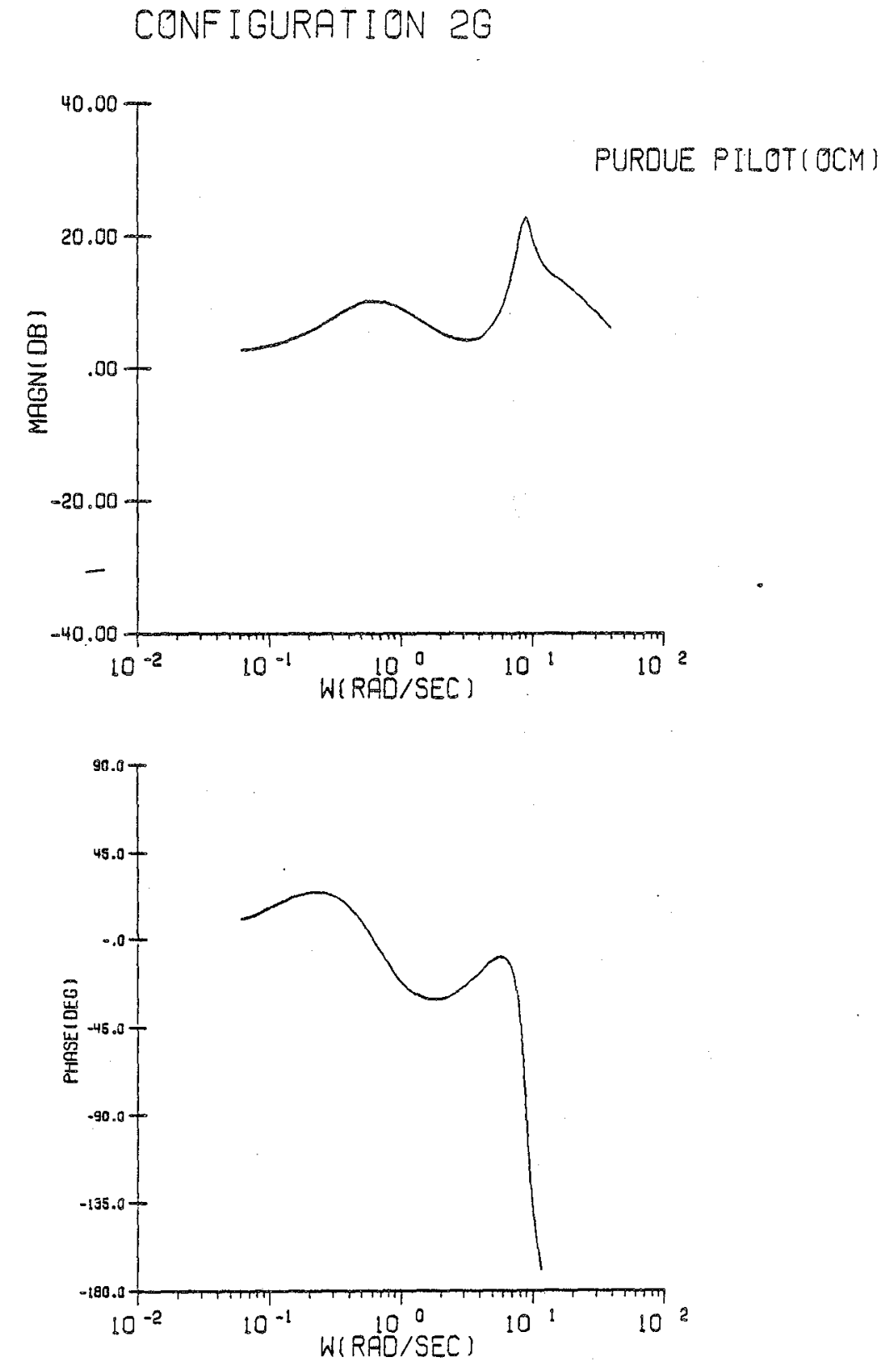

Figure C.38 Configuration $2 \mathrm{G} / \mathrm{Pi}$ lot Frequency Response 


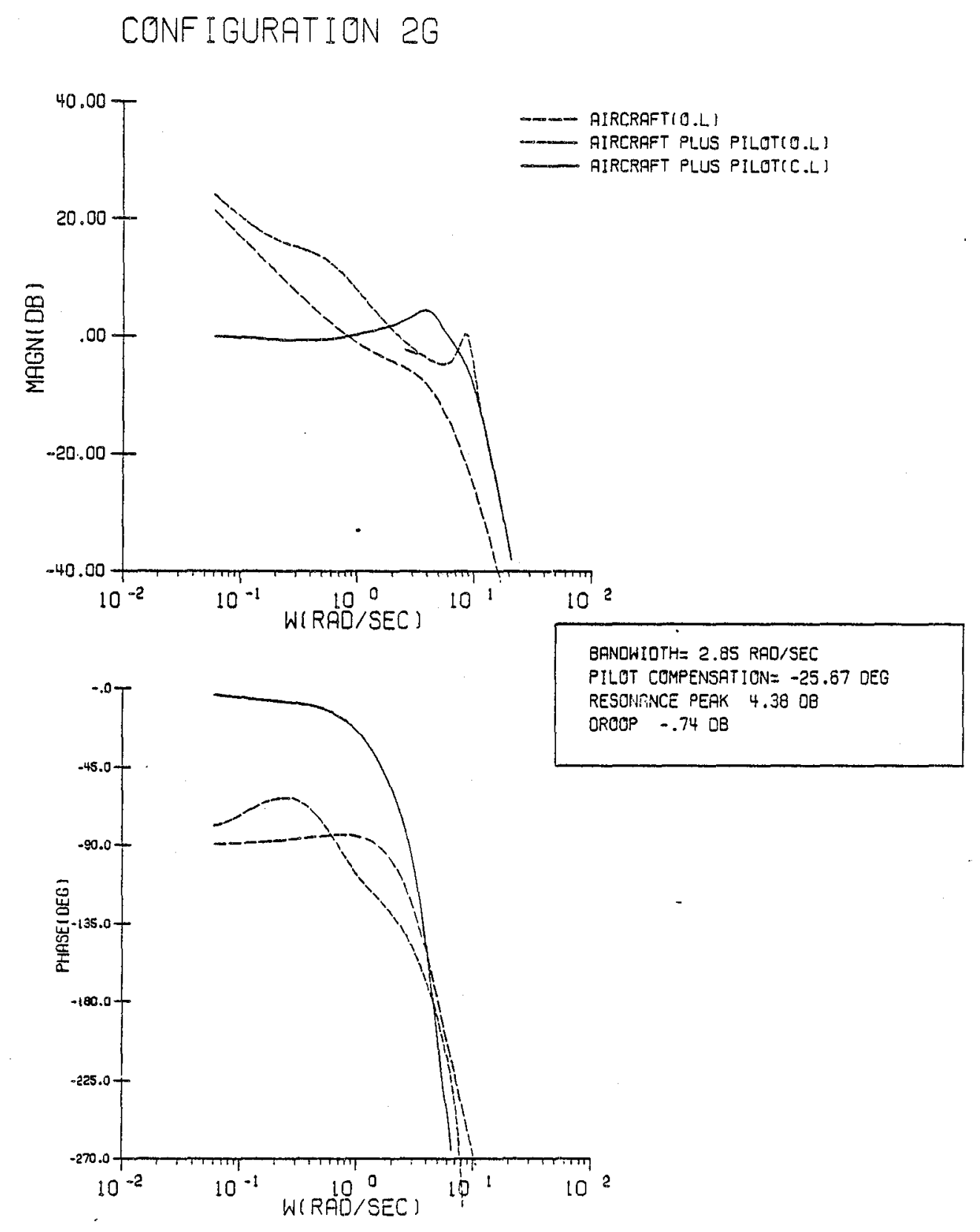

Figure C.39 Configuration 2G/Sys tem Frequency Response 
CONFIGURATION 2G

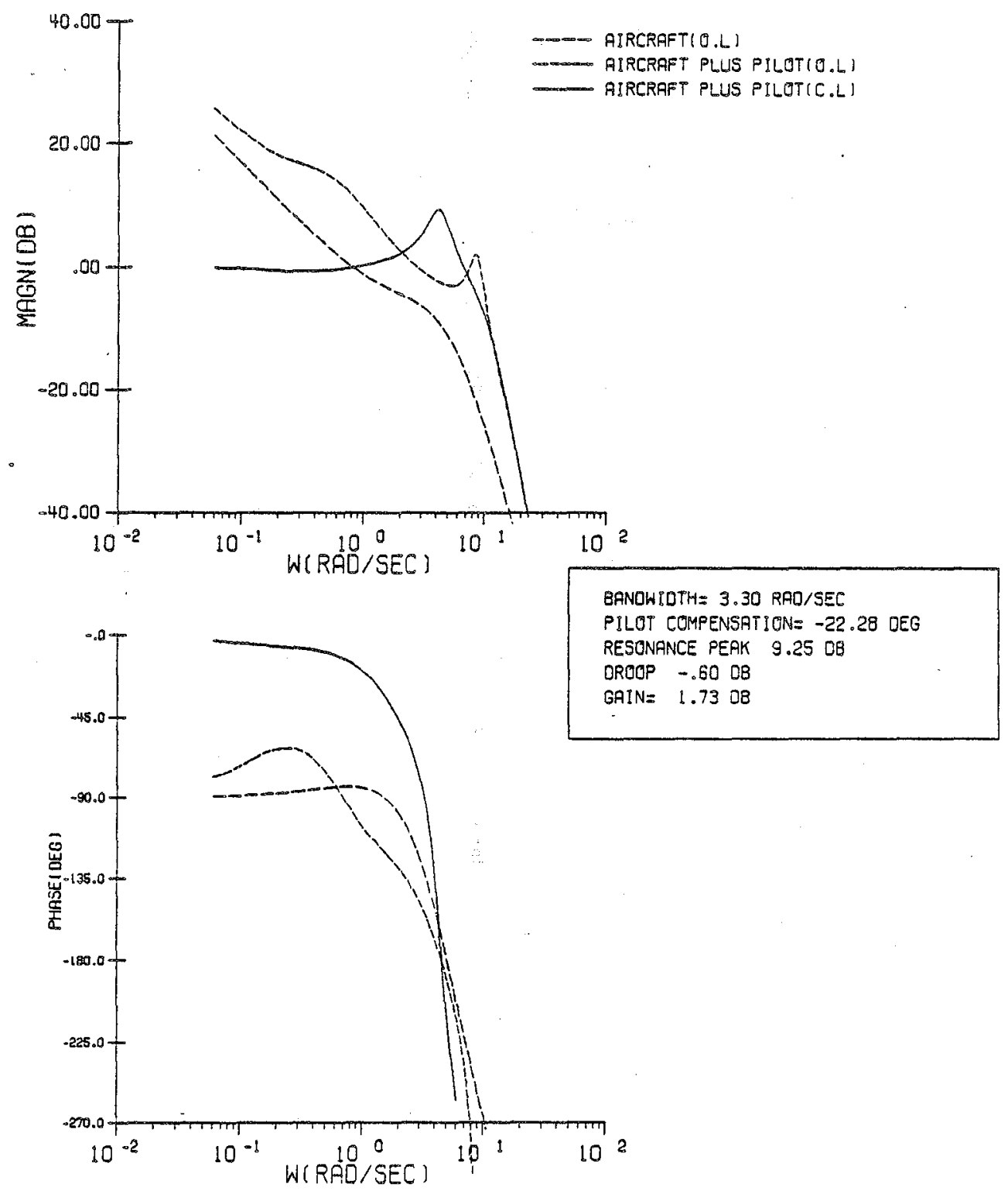

Figure C.40 Configuration 2G/Corrected System Frequency Response 

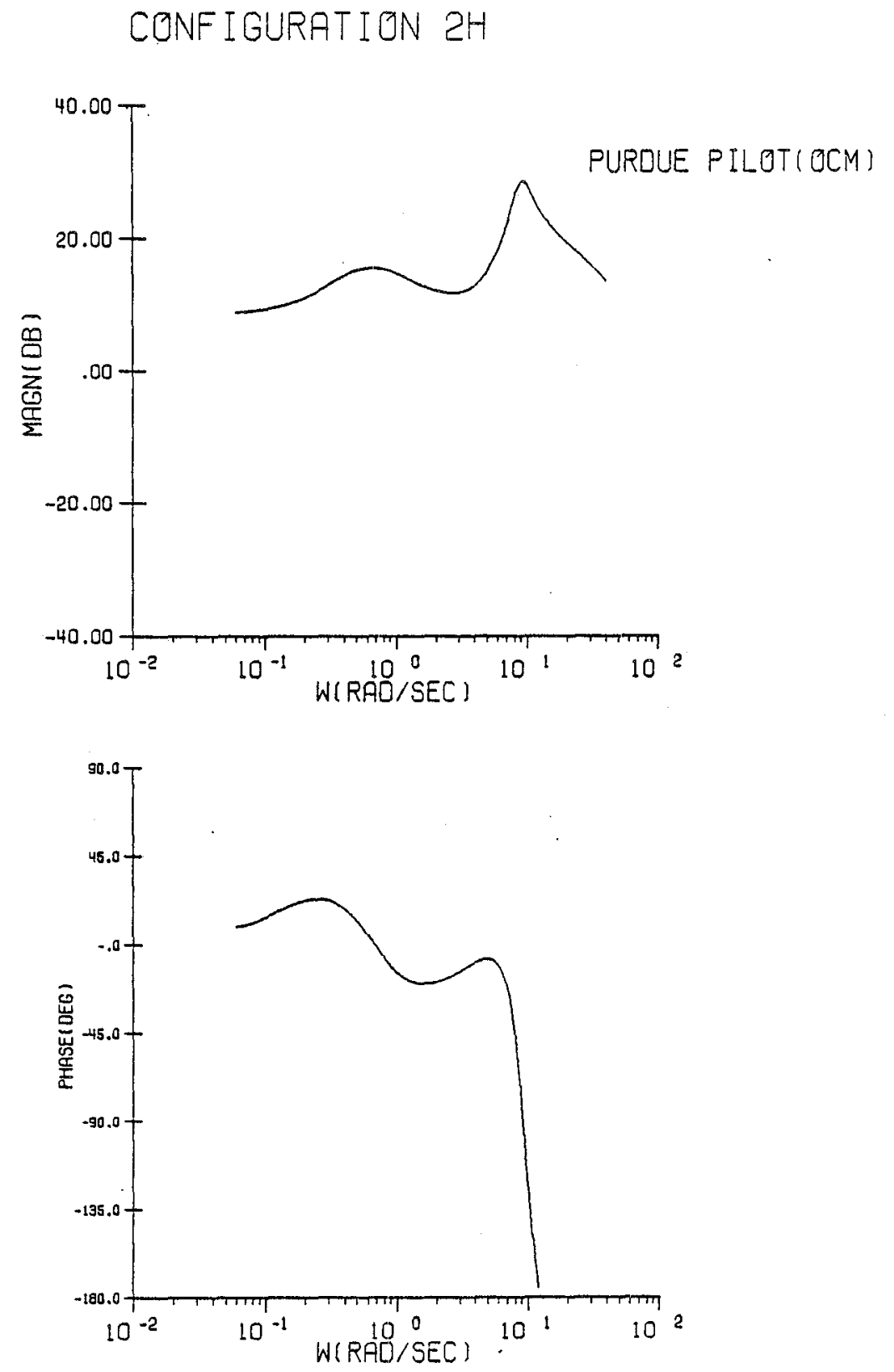

Figure C.41 Configuration 2H/Pilot Frequency Response 
CONFIGURATION $2 \mathrm{H}$

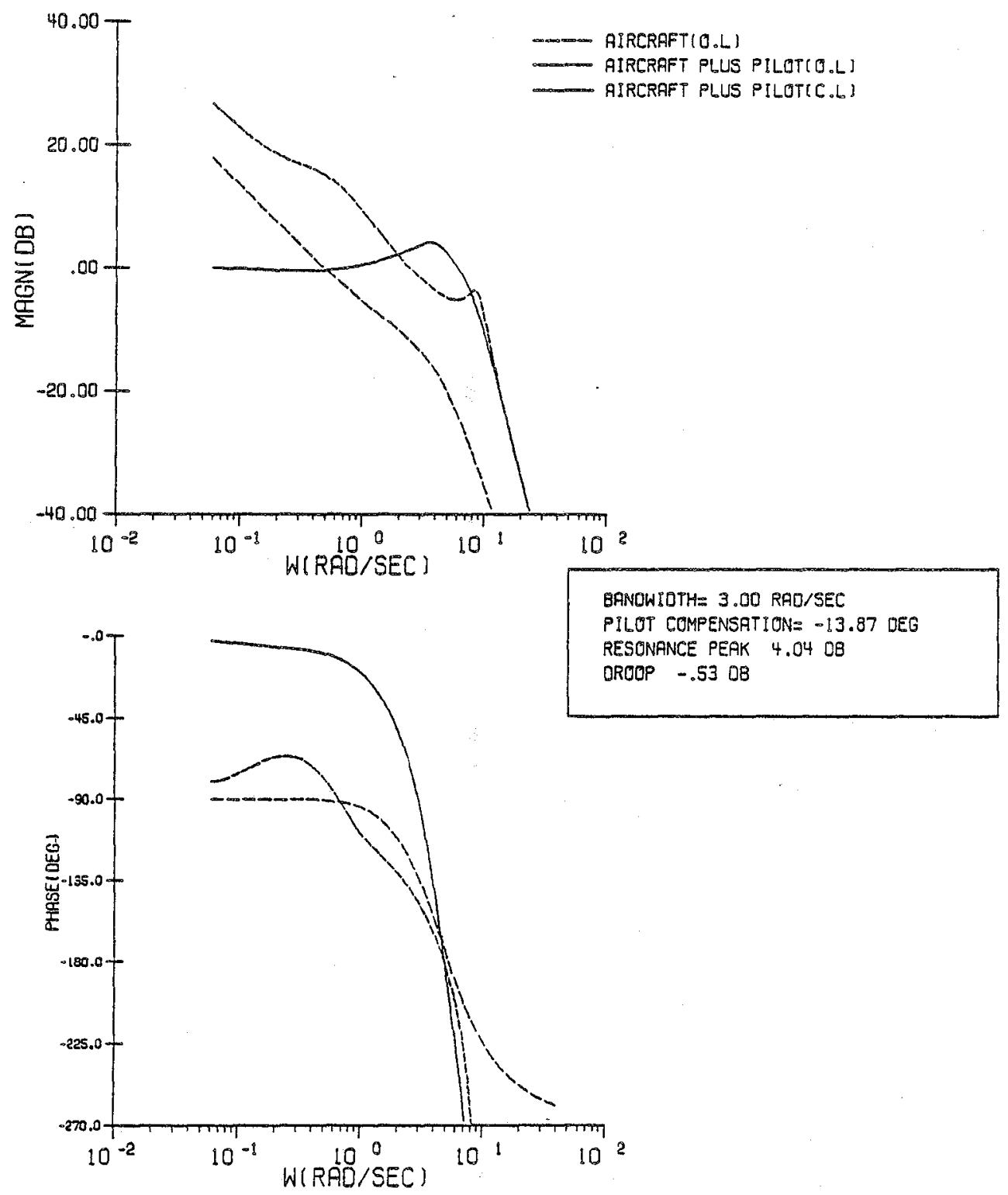

Figure C.42 Configuration 2H/Sys tem Frequency Response 

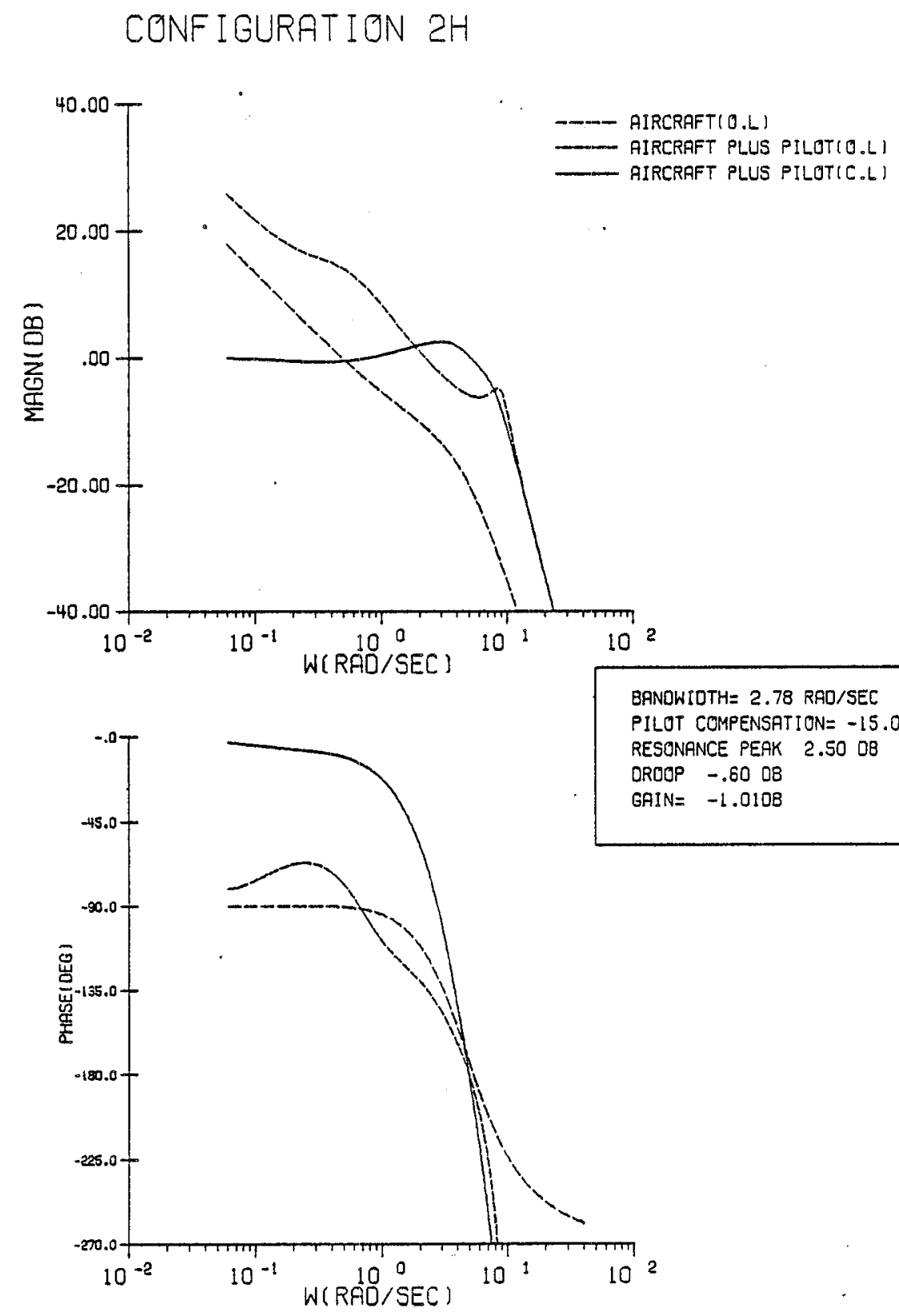

BANOWIDTH $=2.78 \mathrm{RAD} / \mathrm{SEC}$

PILOT COMPENSATION $=-15.09$ DEG

RESONANCE PEAK 2.50 DB

OROOP.$- .60 \mathrm{DB}$

GAIN $=-1.0108$

Figure C.43 Configuration $2 \mathrm{H} /$ Corrected System Frequency Response 
CONFIGURATION ZI
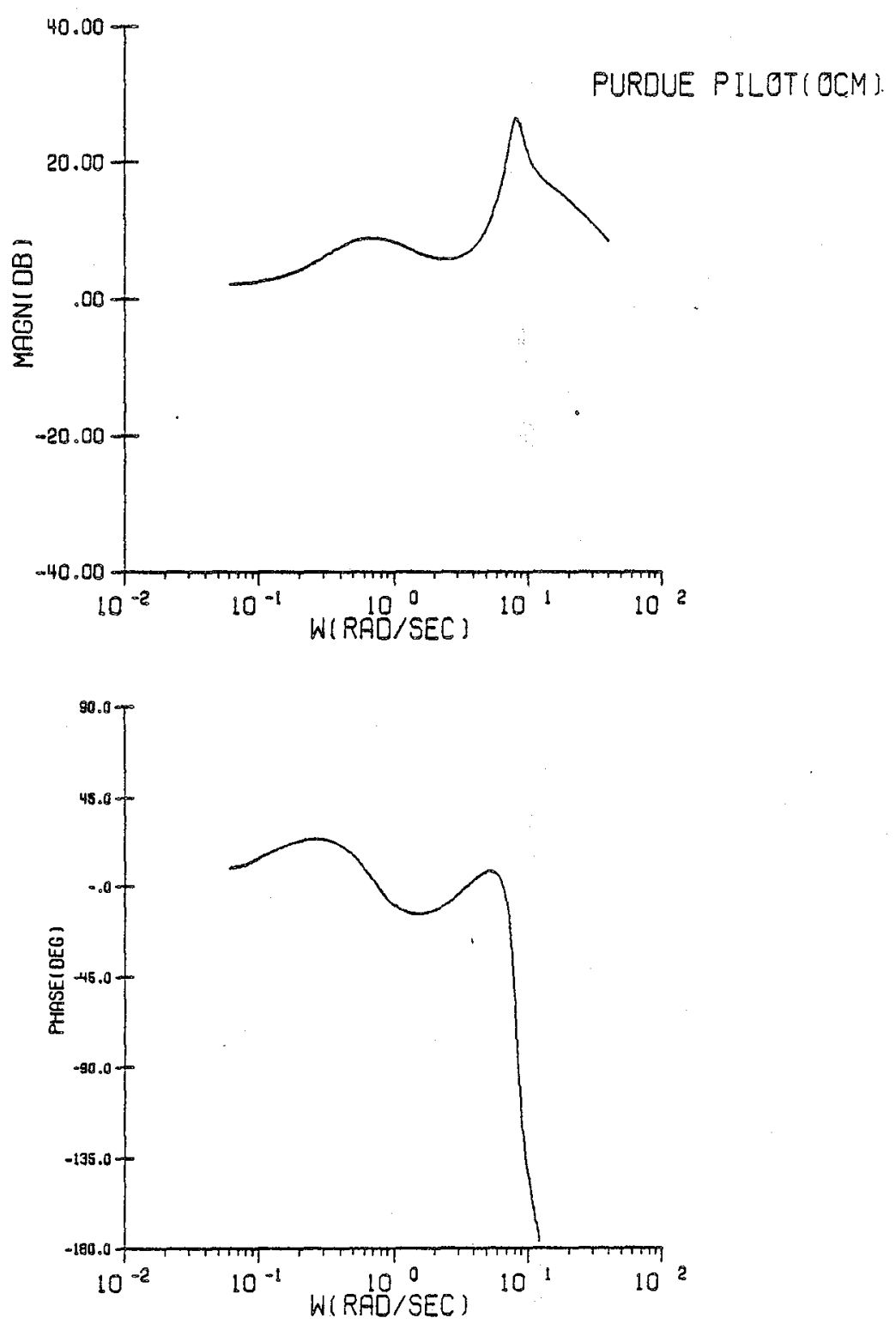

Figure C.44 Configuration 2I/Pilot Frequency Response 


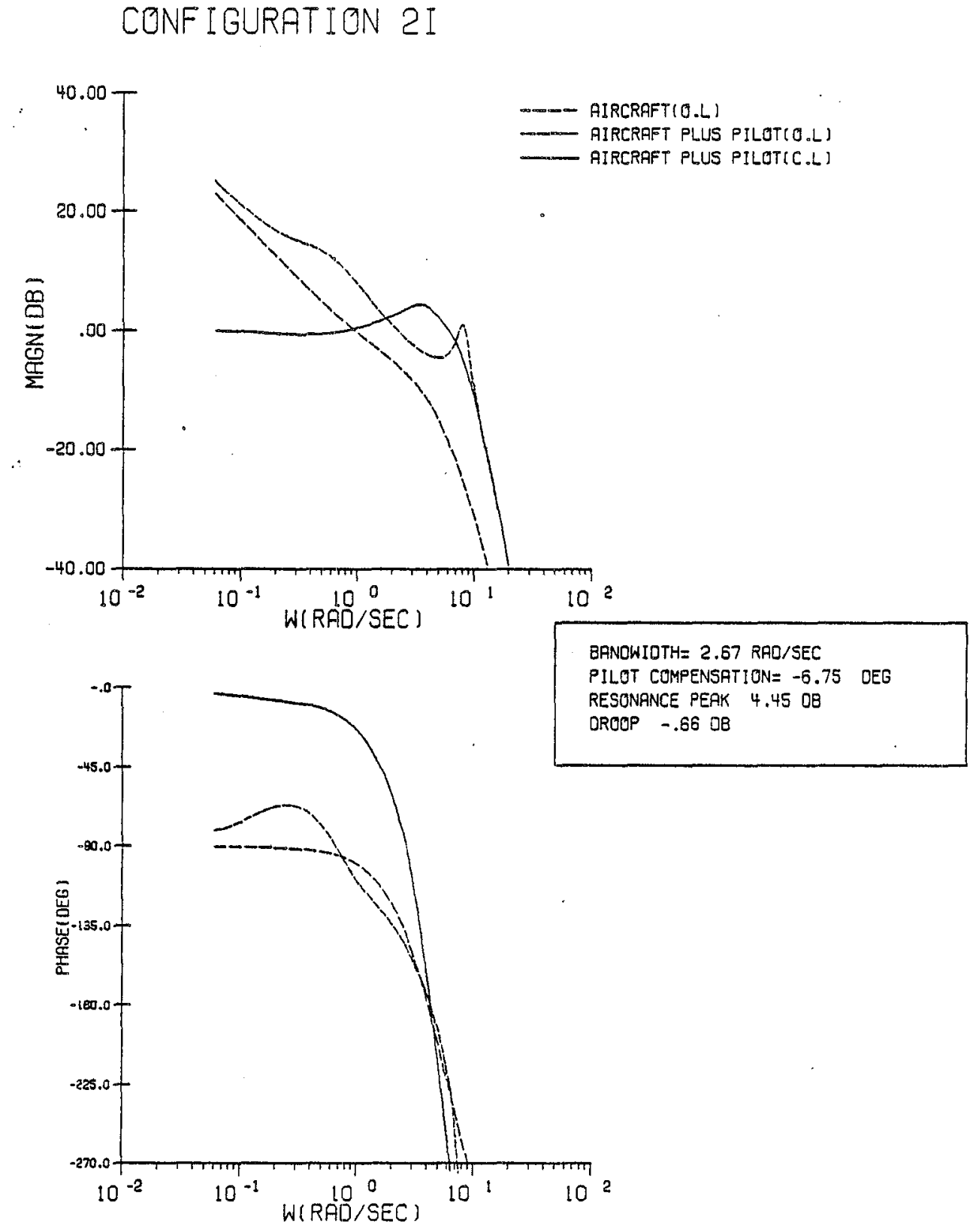

Figure C.45 Configuration 2I/System Frequency Response 
CONFIGURATION $2 I$

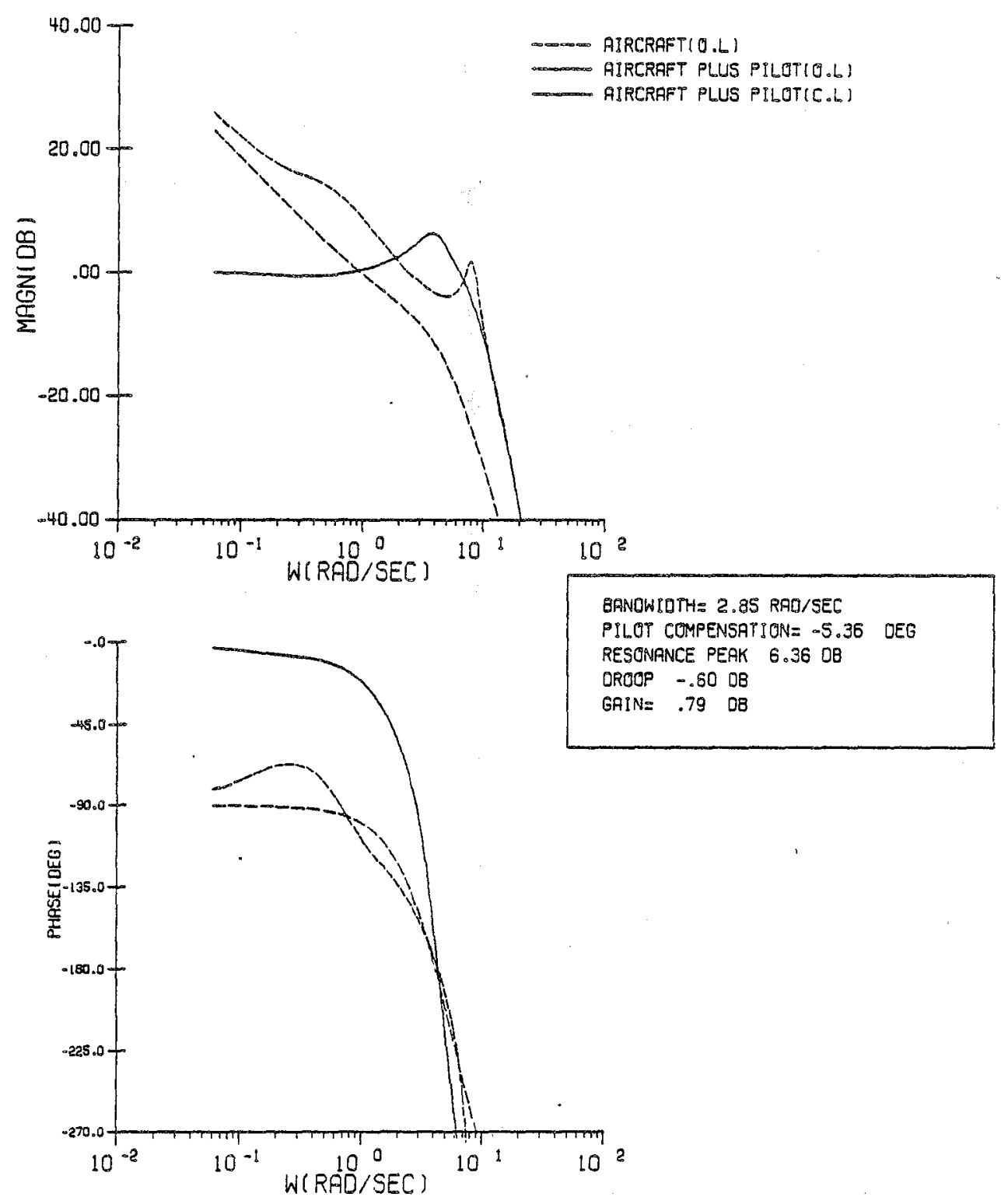

Figure C.46 Configuration 2I/Corrected System Frequency Response 
CONFIGURATION $2 \mathrm{~J}$
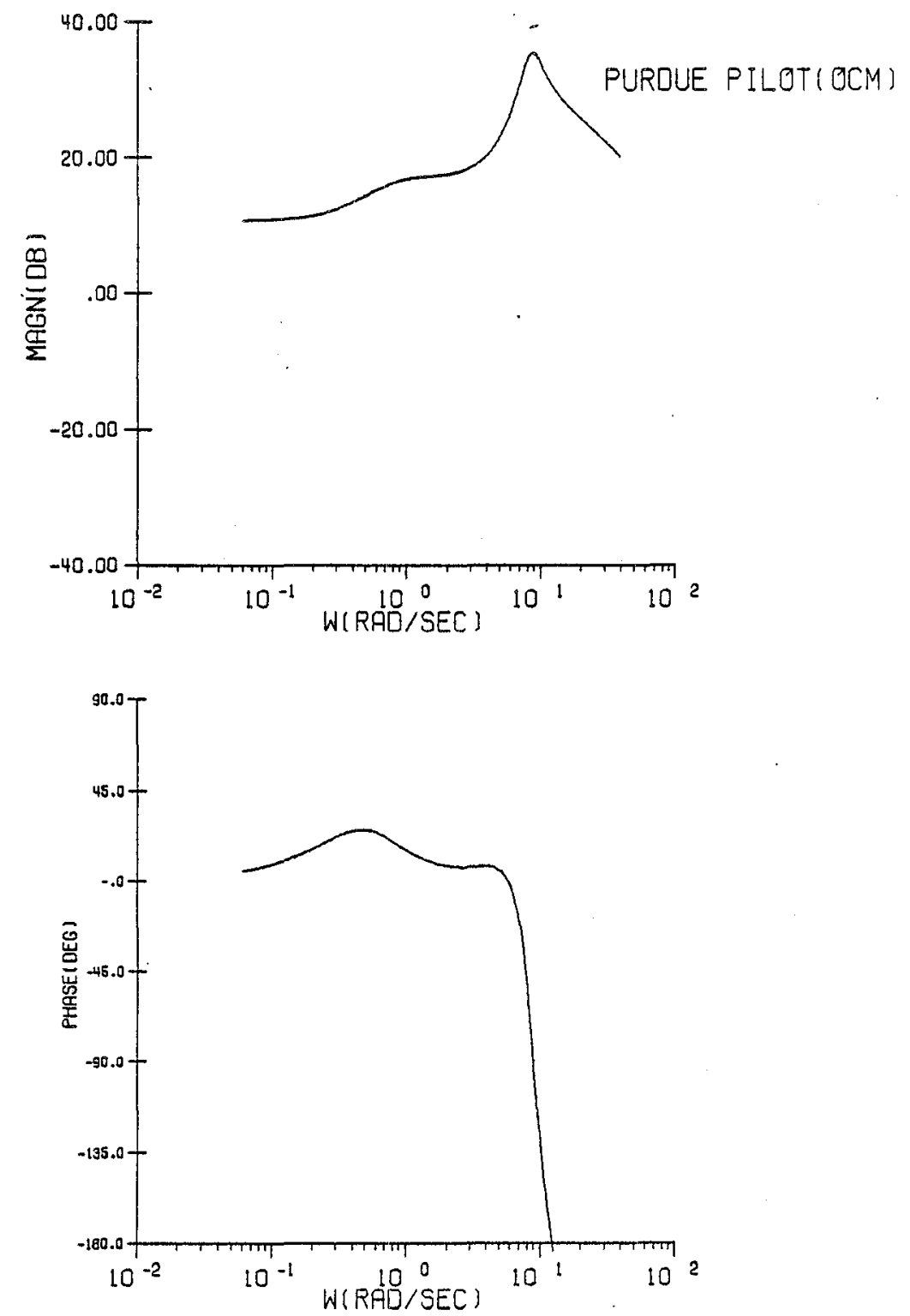

Figure C.47 Configuration 2J/Pilot Frequency Response 
CONF IGURATION $2 \mathrm{~J}$

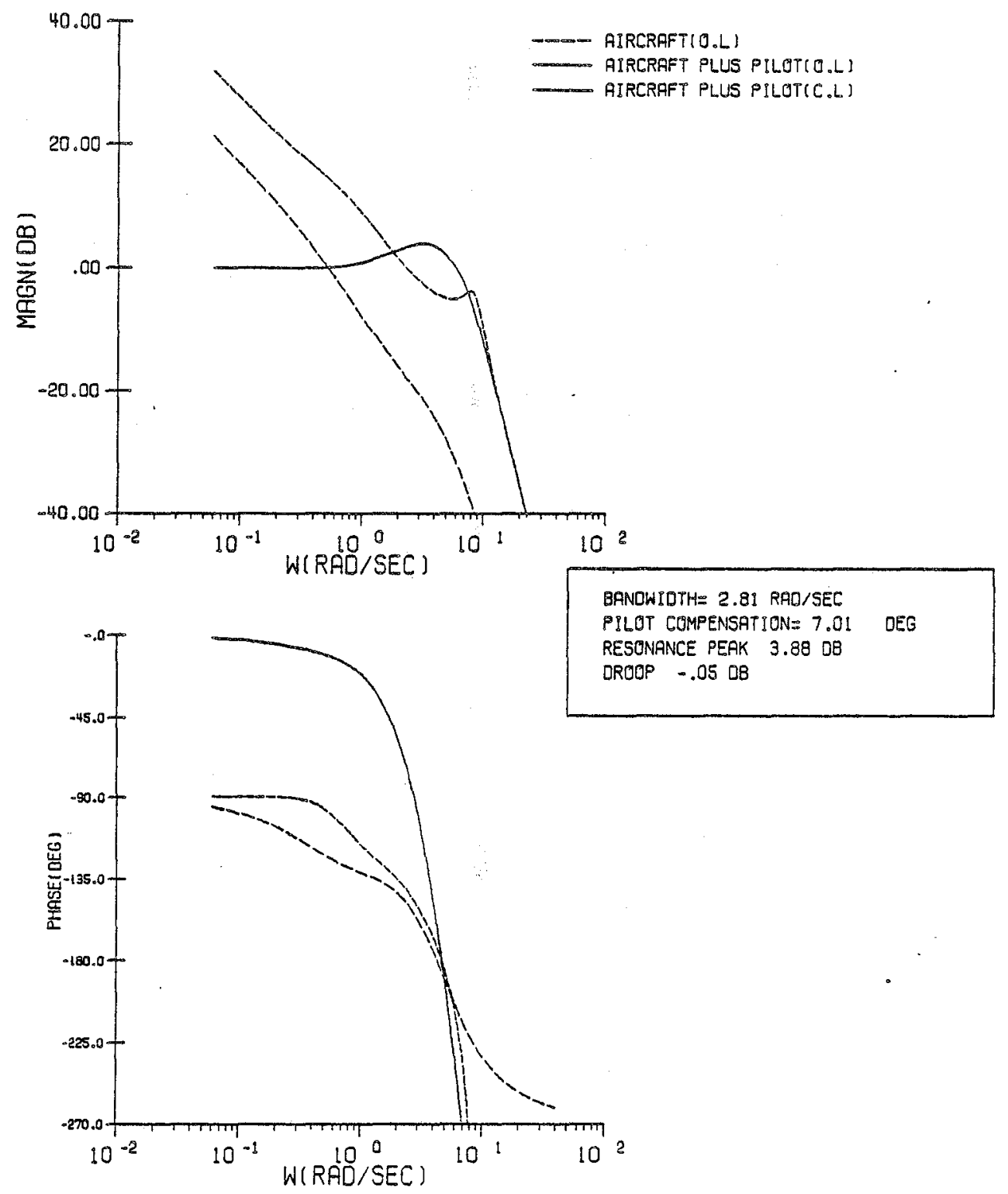

Figure C.48 Configuration 2J/System Frequency Response 
CONFIGURATION 3A
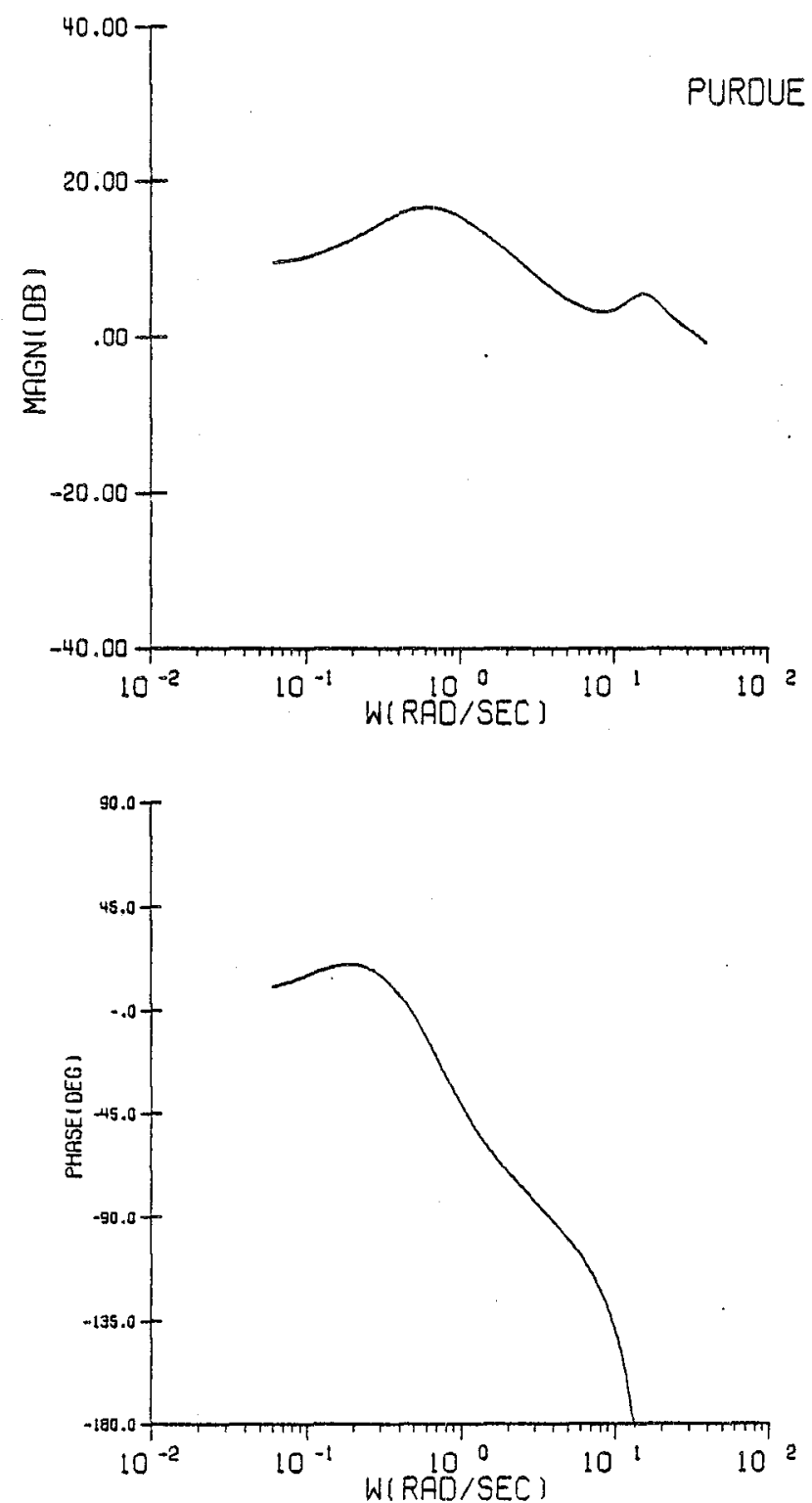

Figure C. 49 Configuration $3 \mathrm{~A} / \mathrm{Pi}$ lot Frequency Response 


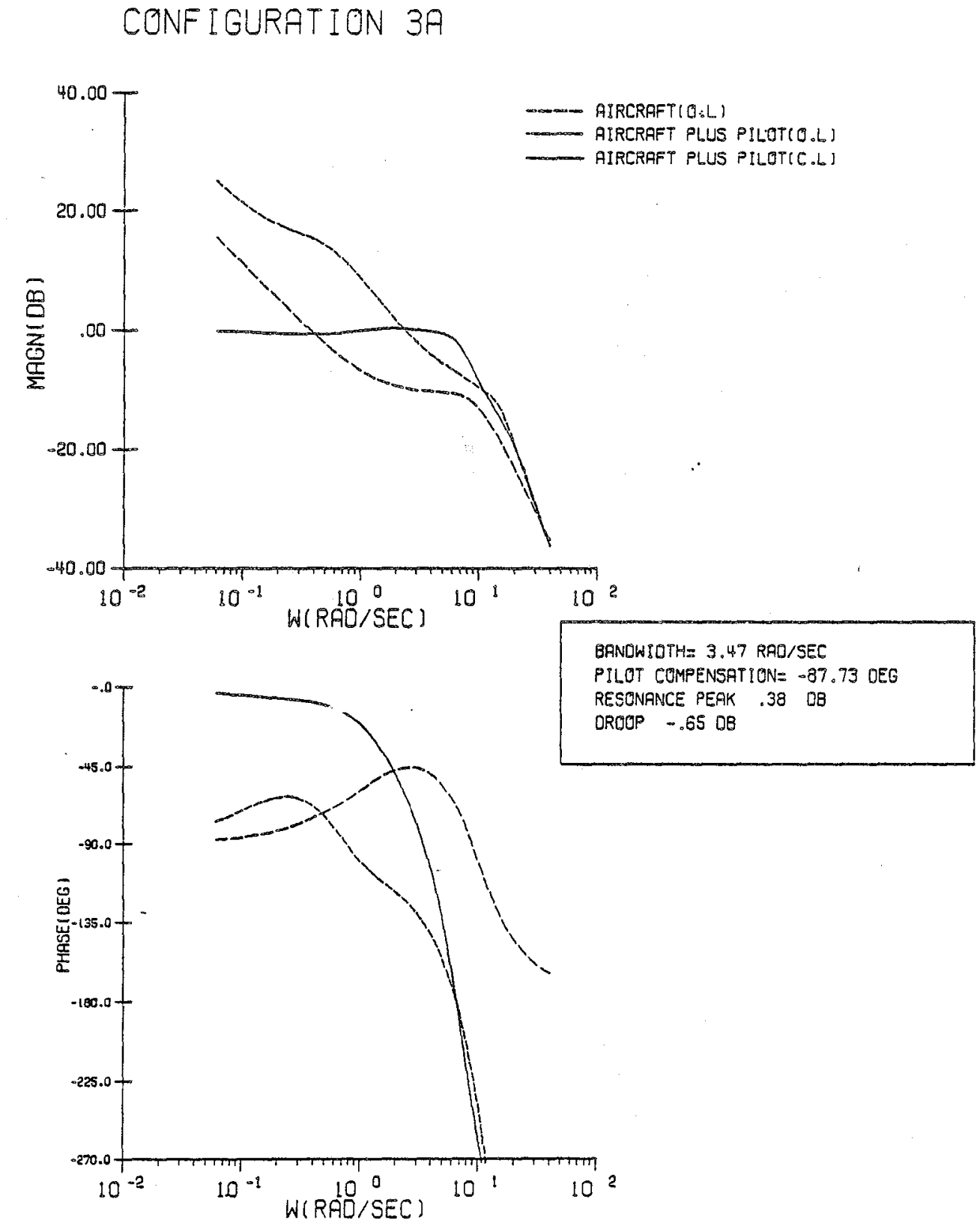

Figure C.50 Configuration $3 \mathrm{~A} /$ Sys tem Frequency Response 
CONFIGURATION 3A

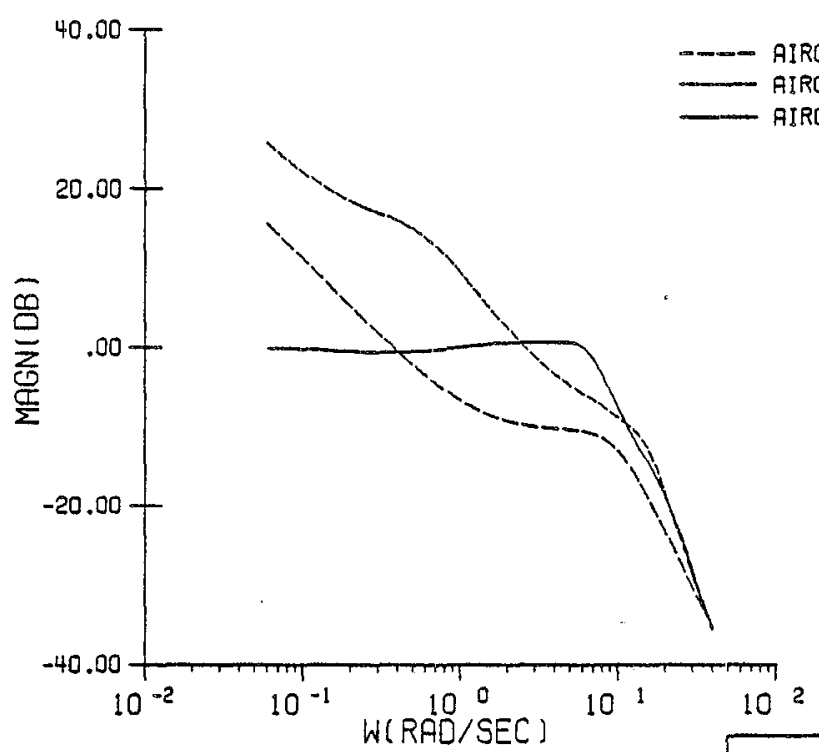

BANOWLDTH= 3.65 RAD/SEC

PILOT COMPENSATION $=-89.29$ DEG

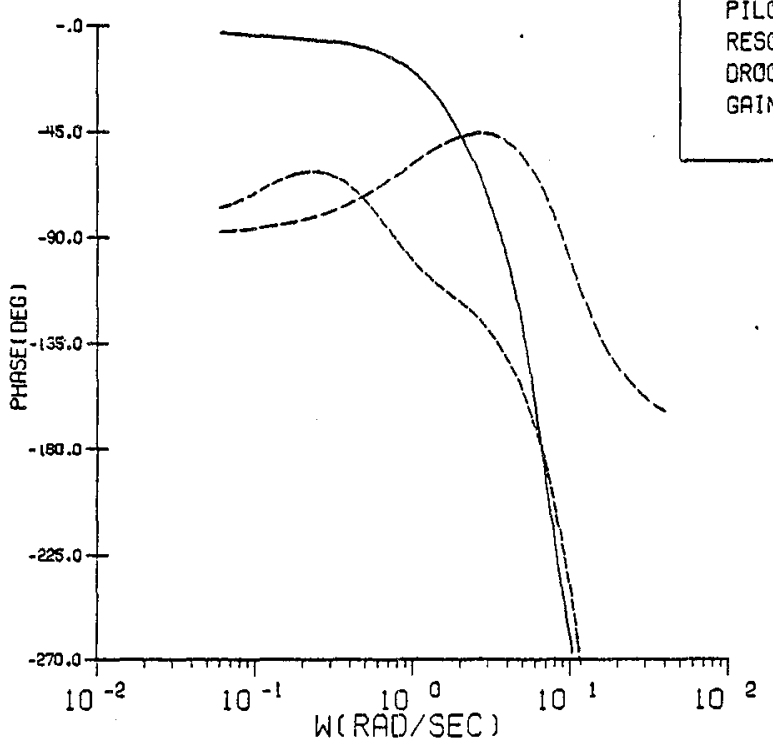

Figure C.51 Configuration 3A/Corrected System Frequency Response 
CONFIGURATION $4 \mathrm{~A}$
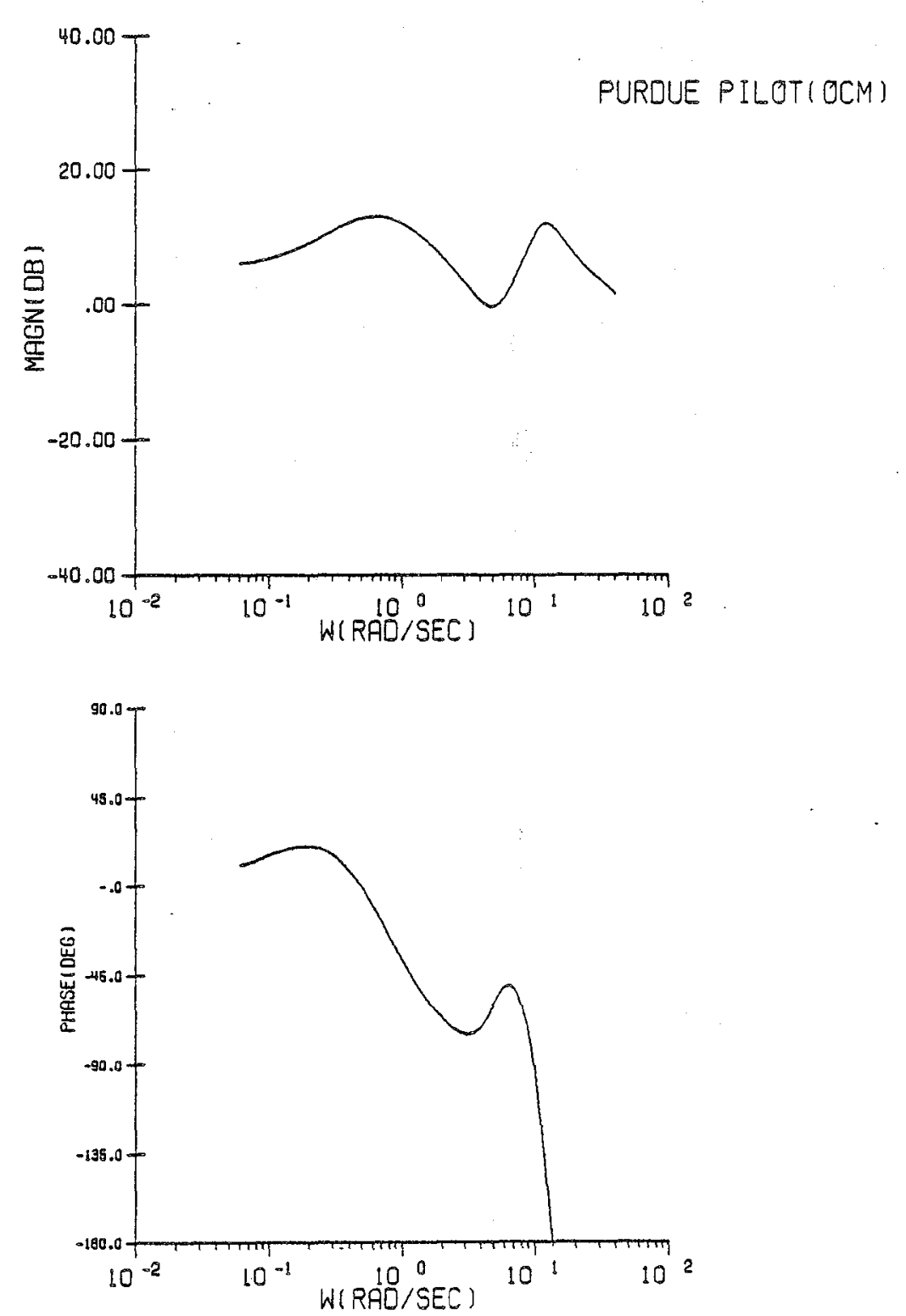

Figure C.52 Configuration 4A/Pilot Frequency Response 
CONFIGURATION $4 \mathrm{~A}$

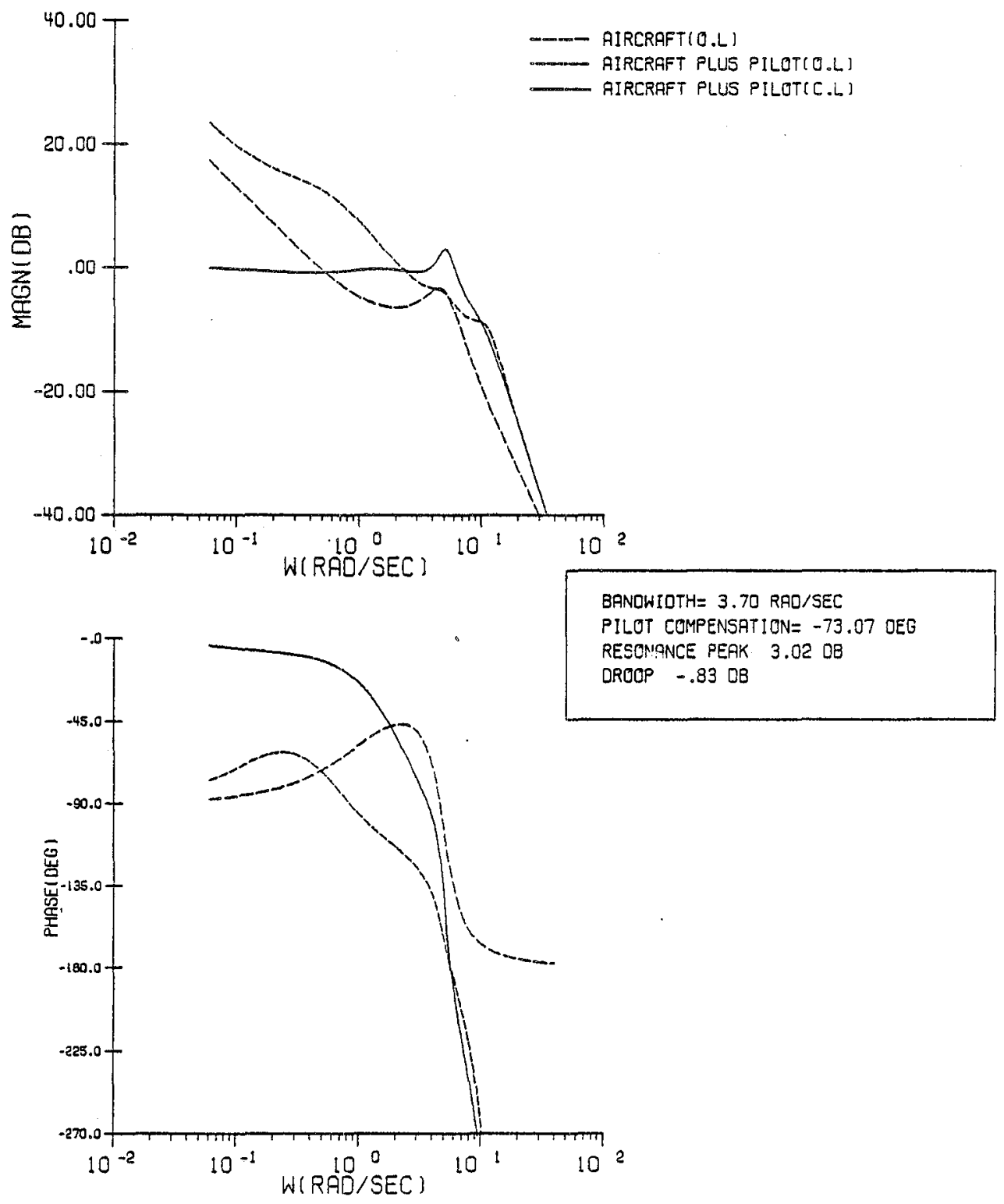

Figure C.53 Configuration 4A/System Frequency Response 
CONFIGURATION 4 A

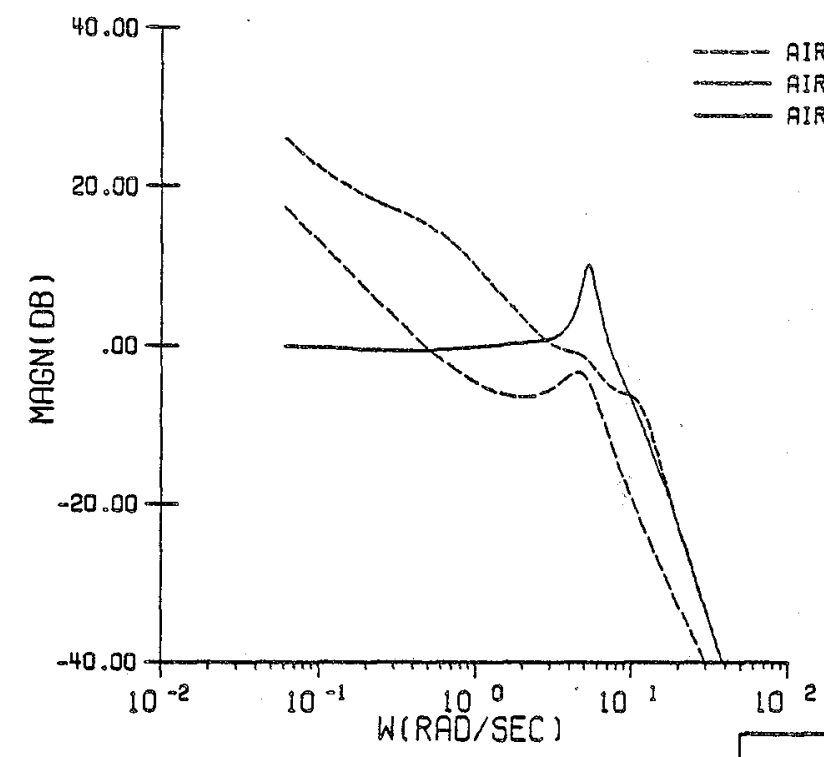

BANOW IOTH= 4.62 RAO/SEC

PILOT COMPENSATION= -63.81 OEG

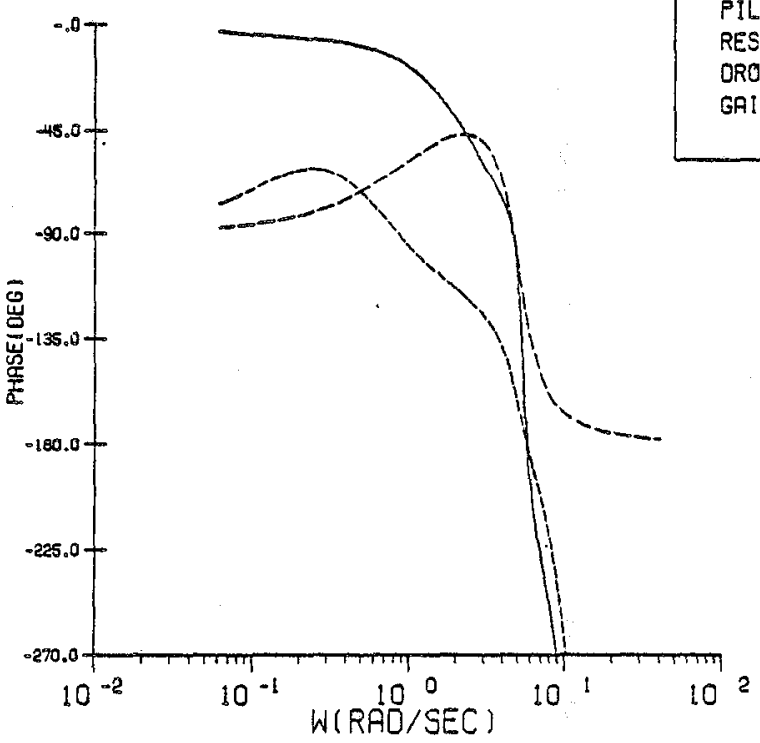

Figure C.54 Configuration $4 A /$ Corrected System Frequency Response 
CONF IGURATION 5A
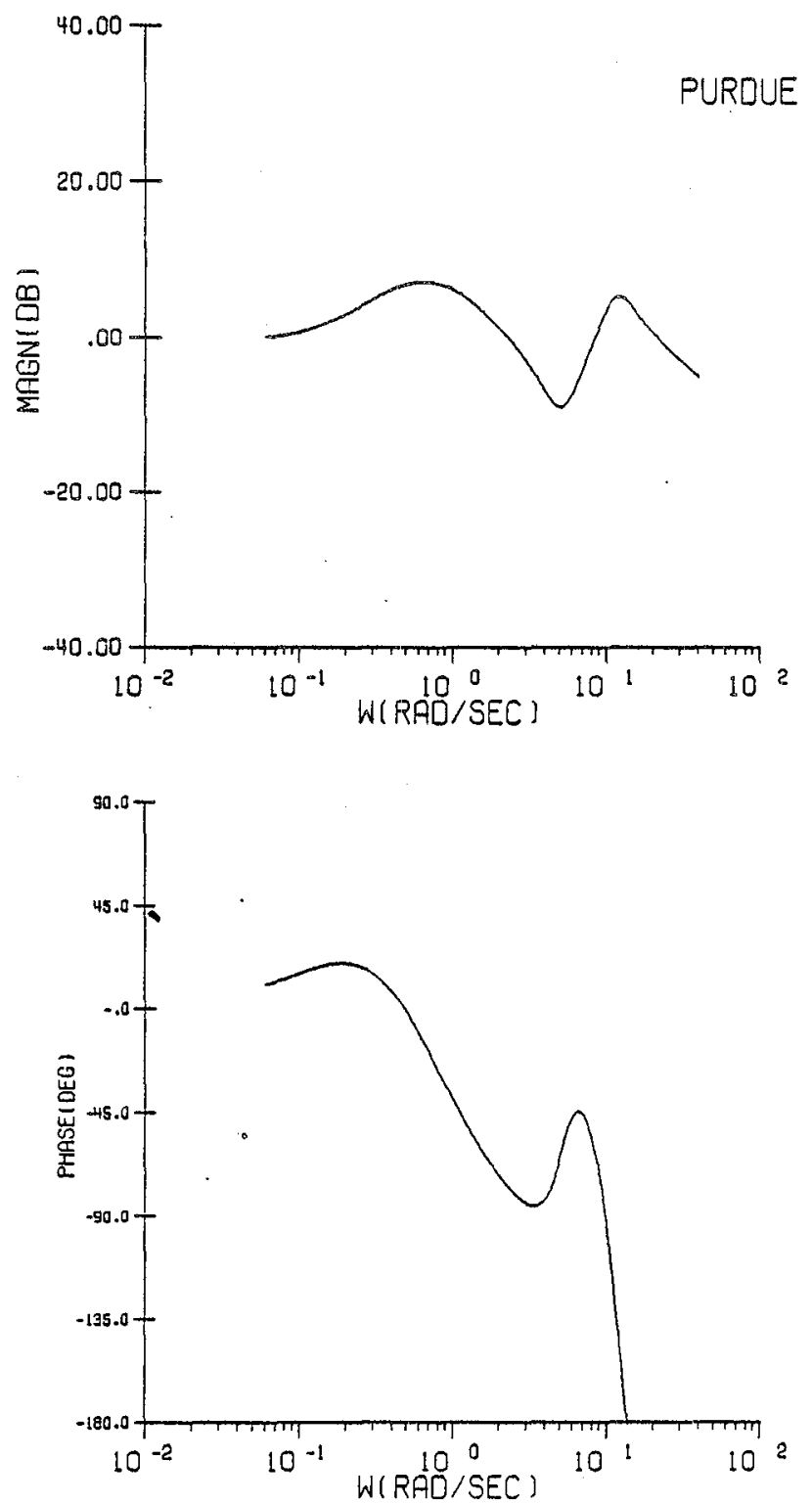

Figure C.55 Configuration 5A/Pilot Frequency Response 


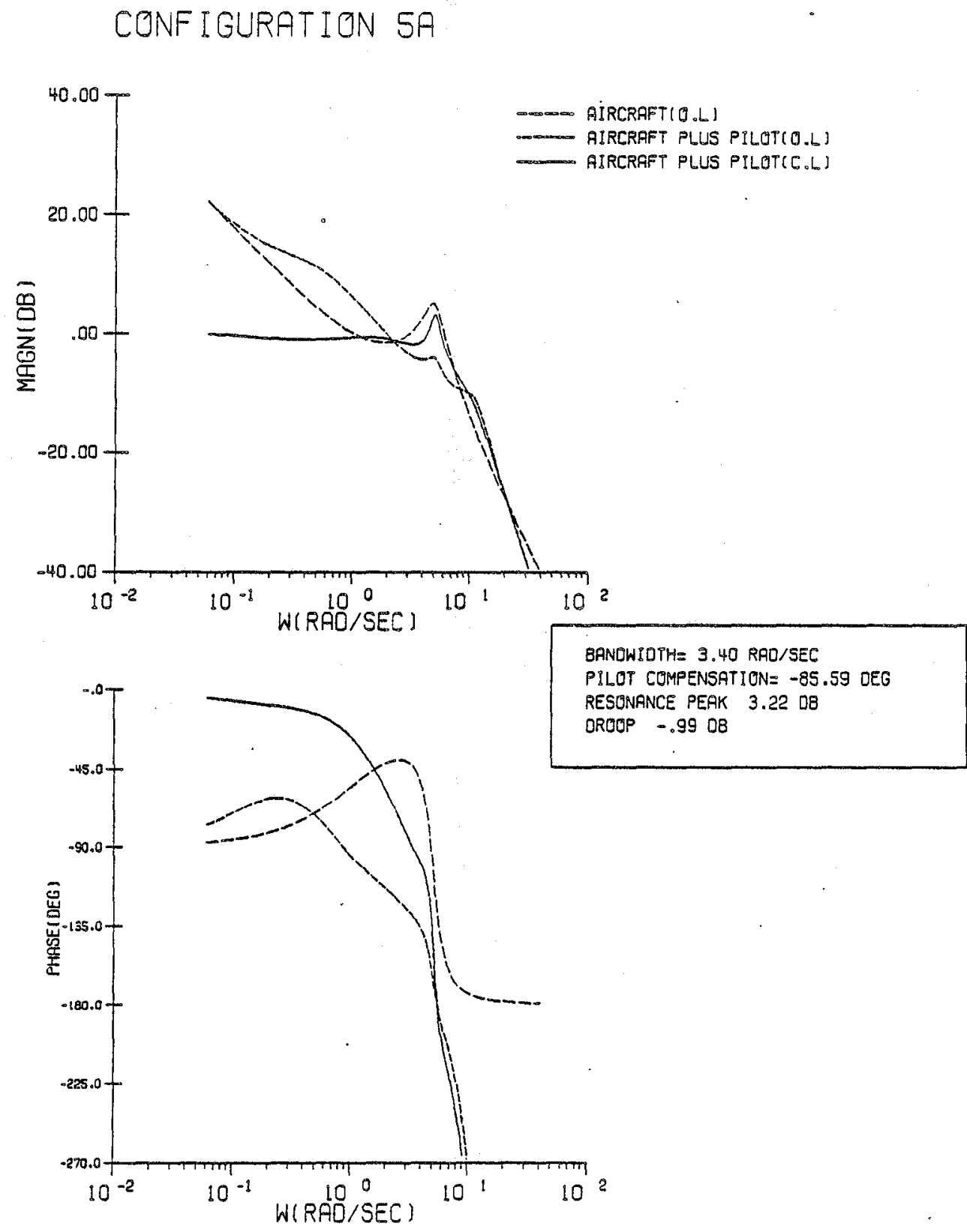

Figure C.56 Configuration 5A/System Frequency Response 
CONF IGURATION 5 A

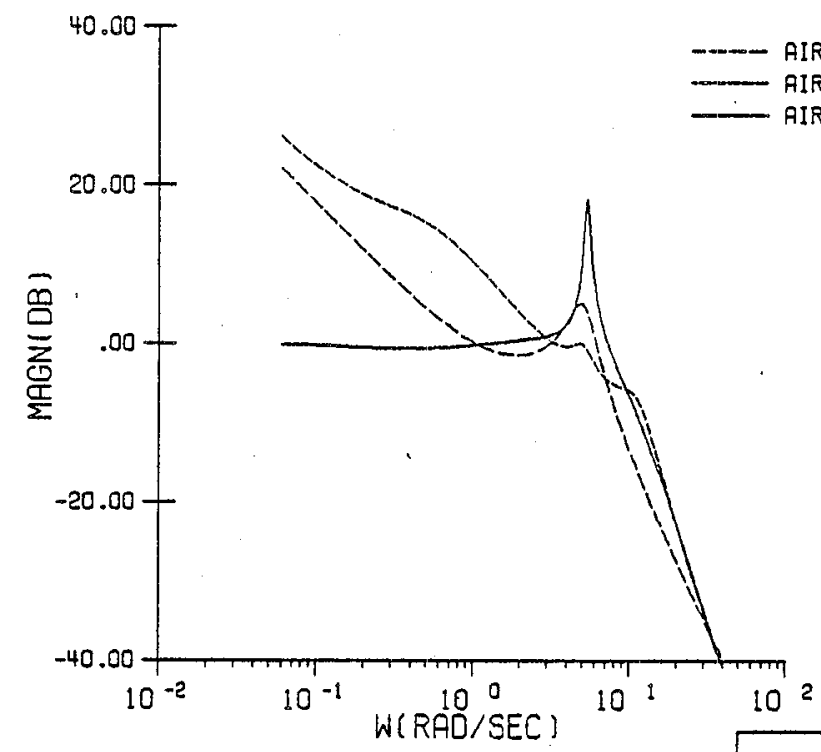

BRNOWLDTH $=5.17$ RAD/SEC

PILOT COMPENSATION $=-60.56$ OEG

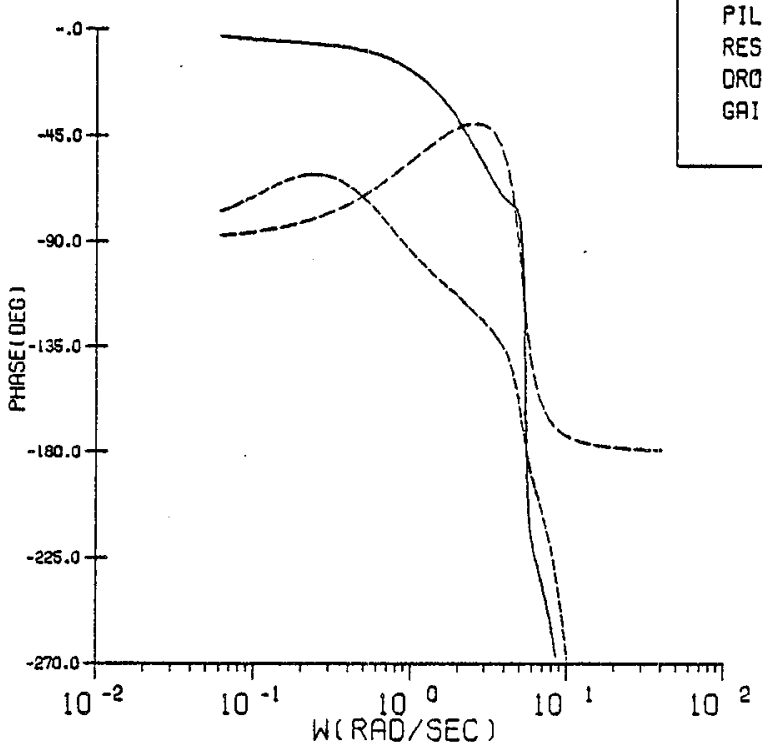

Figure C.57 Configuration 5A/Corrected System Frequency Response 
CONFIGURATION $6 \mathrm{C}$
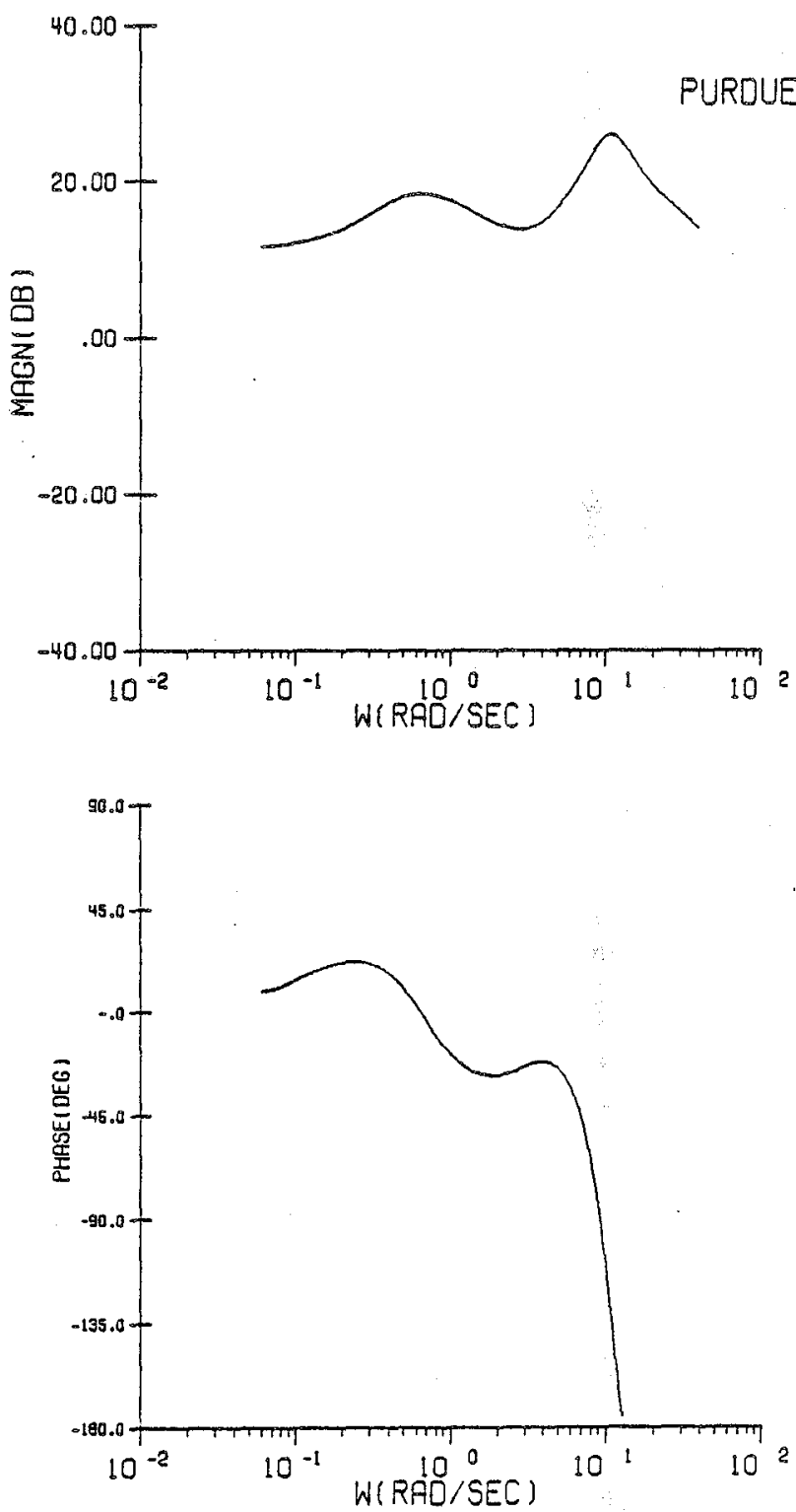

Figure C.58 Configuration $6 \mathrm{C} / \mathrm{Pi}$ lot Frequency Response 
CONF IGURATION GC

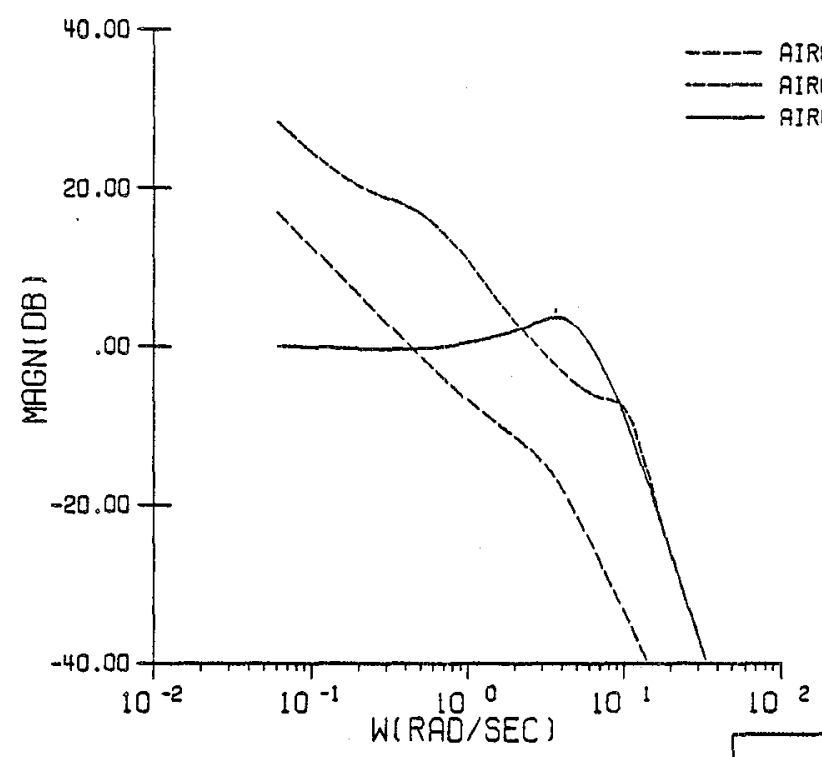

BANOWIDTH $=3.32$ RAO/SEC

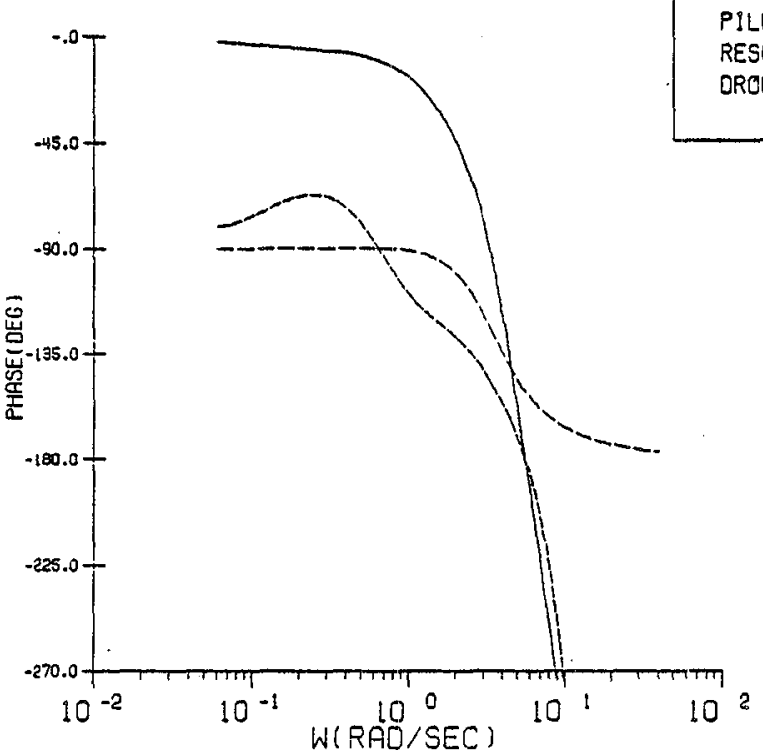

Figure C.59 Configuration $6 \mathrm{C} /$ System Frequency Response 
CONFIGURATION $6 C$

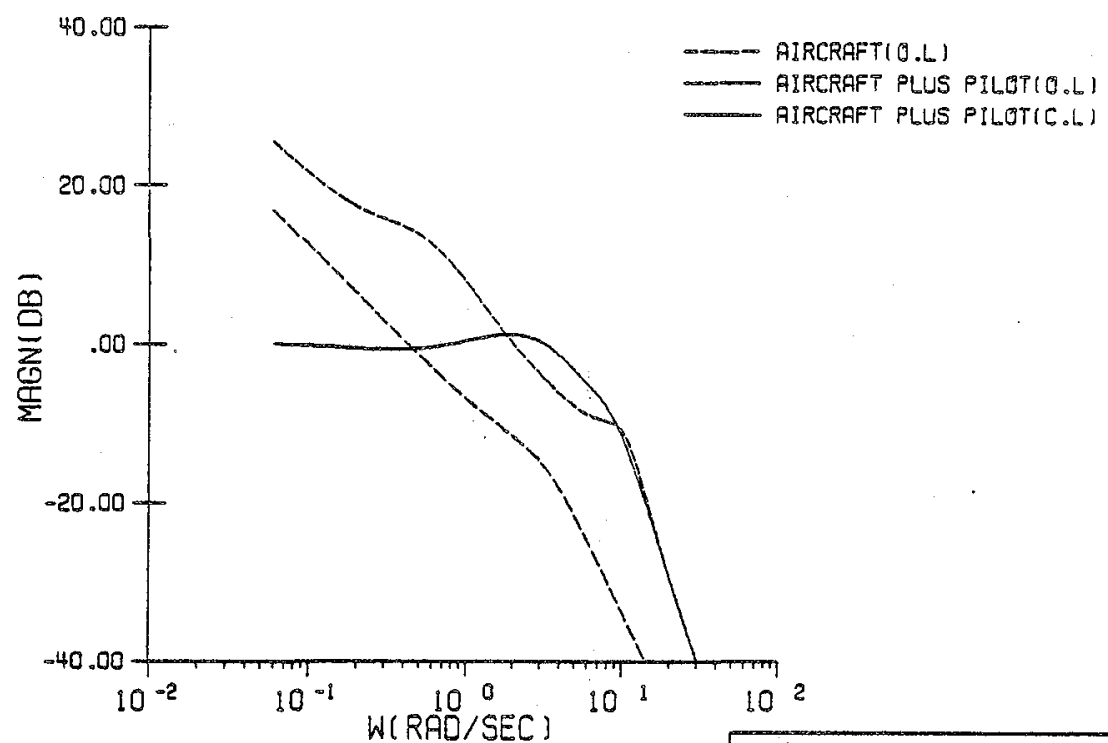

BANDWIDTH $=2.70$ RFD/SEC

PILOT COMPENSATION $=-25.00$ OEG

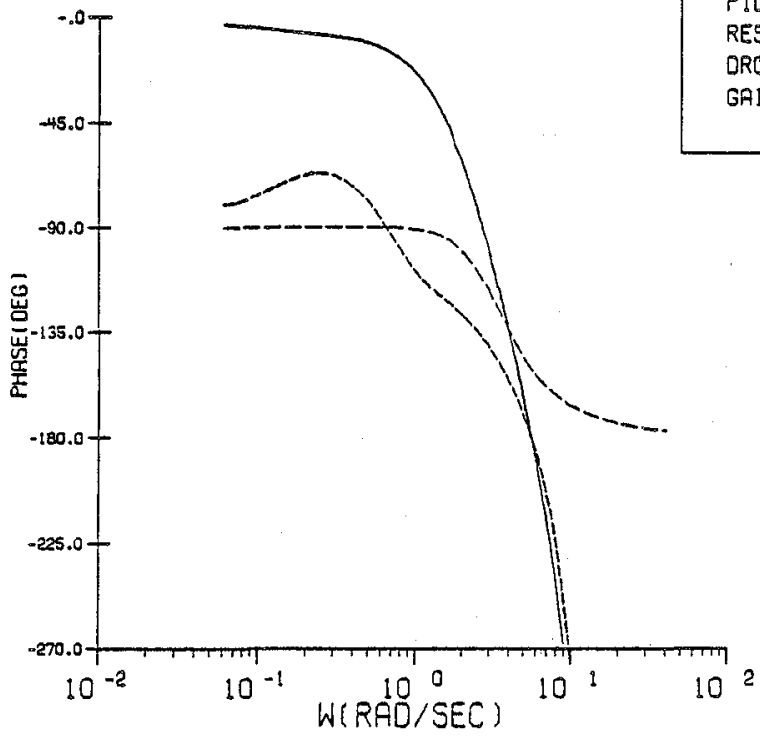

Figure C.60 Configuration $6 C /$ Corrected System Frequency Response 
CONF IGURATION $7 C$

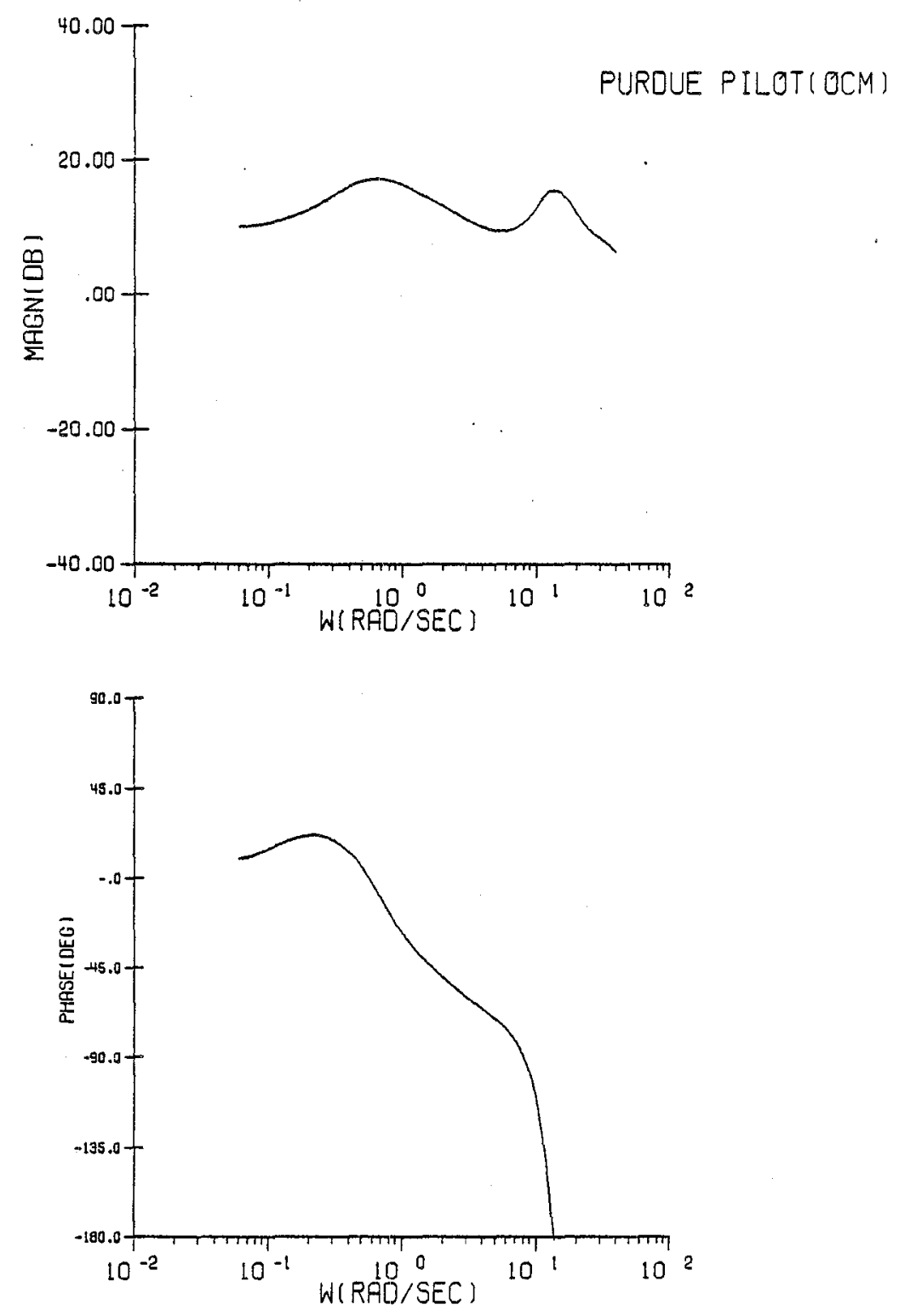

Figure C.61 Configuration $7 C / P i l o t$ Frequency Response 
CONFIGURATION $7 C$

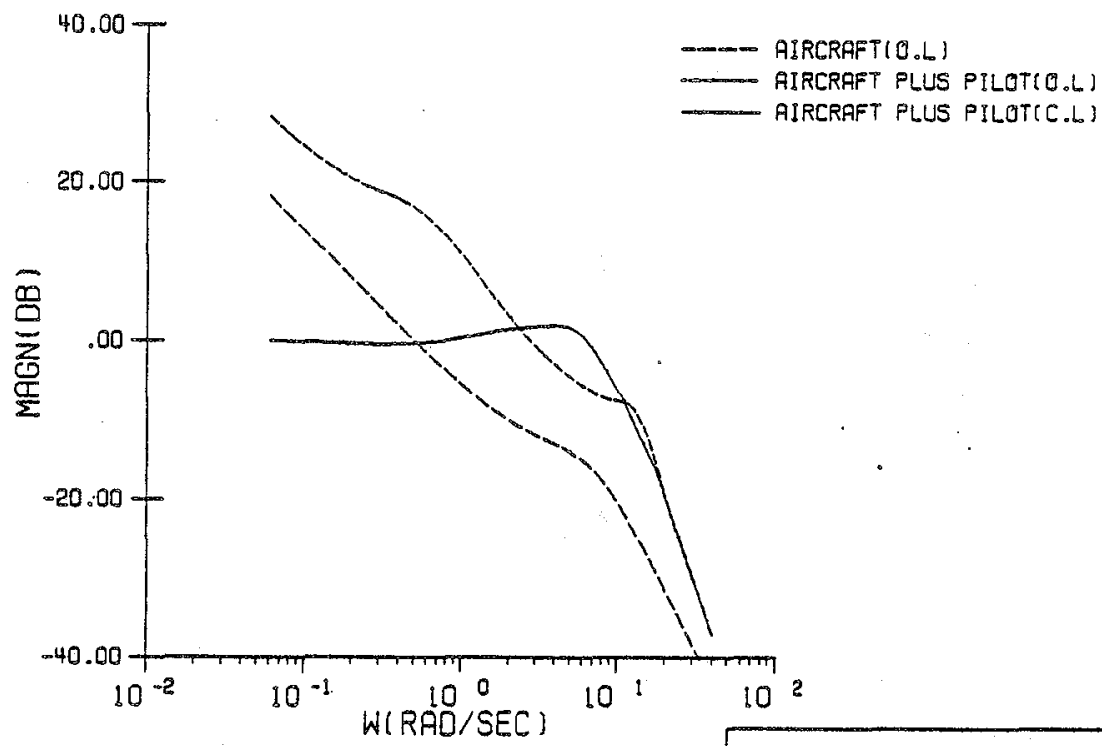

BRNOWIDTH 3.62 RAD/SEC

PYLOT COMPENSATION= -63.58 DEG

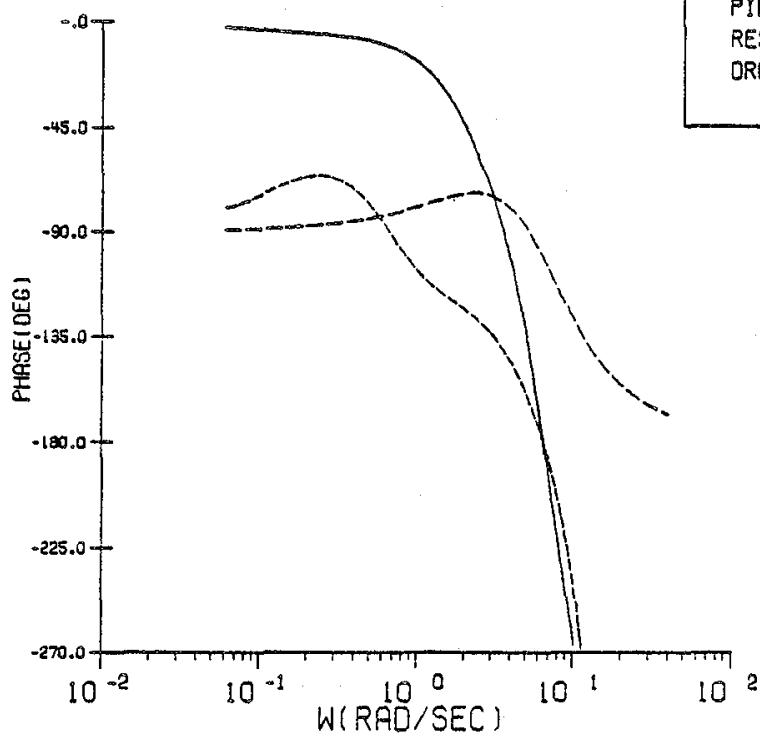

Figure C.62 Configuration $7 C$ /System Frequency Response 


\section{CONFIGURATION $7 C$}

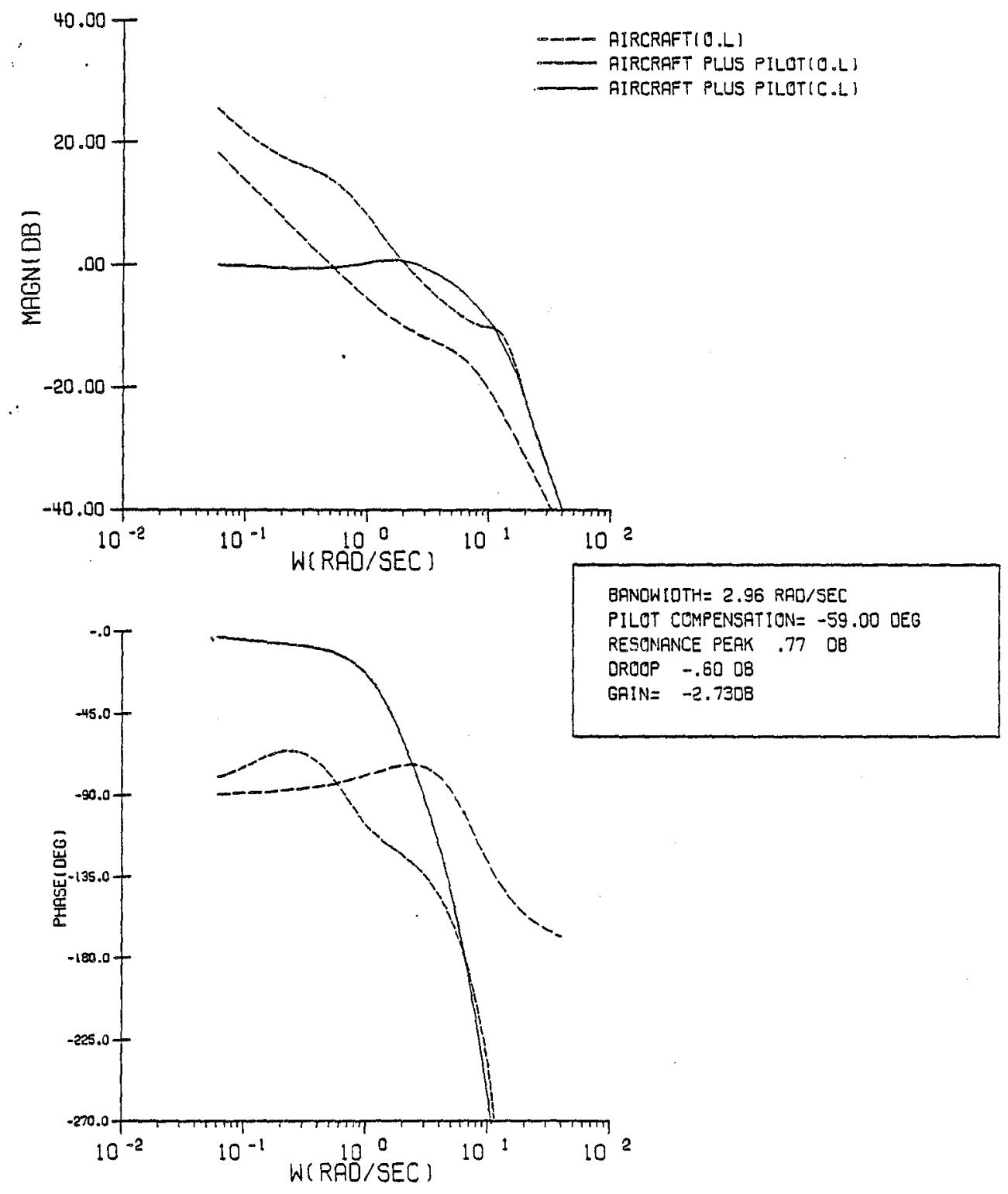

Figure C.63 Configuration $7 C /$ Corrected System Frequency Response 

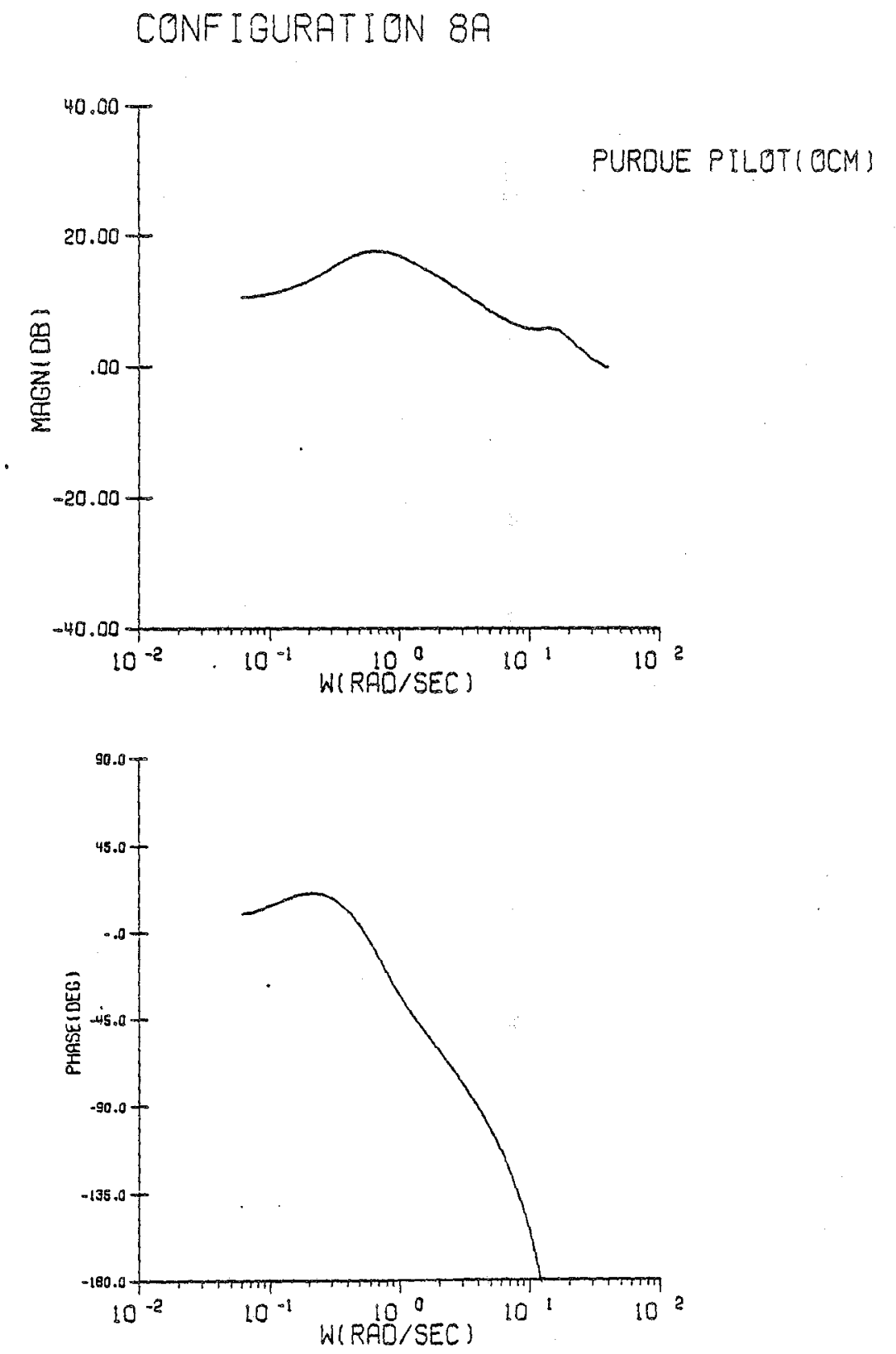

Figure $\mathrm{C} .64$ Configuration $8 \mathrm{~A} / \mathrm{Pi}$ lot Frequency Response 
CONFIGURATION $8 \mathrm{~A}$

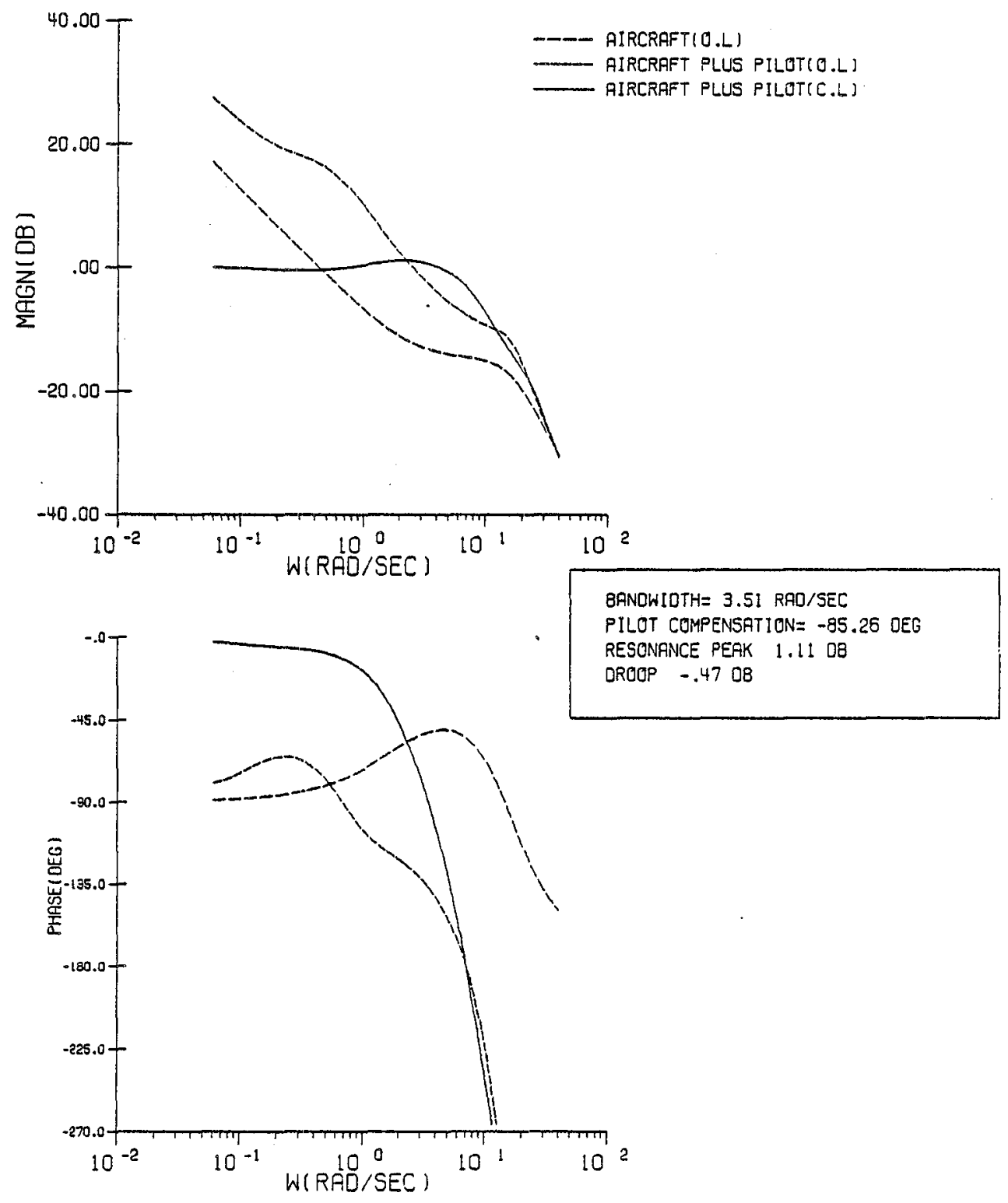

Figure C.65 Configuration 8A/System Frequency Response 


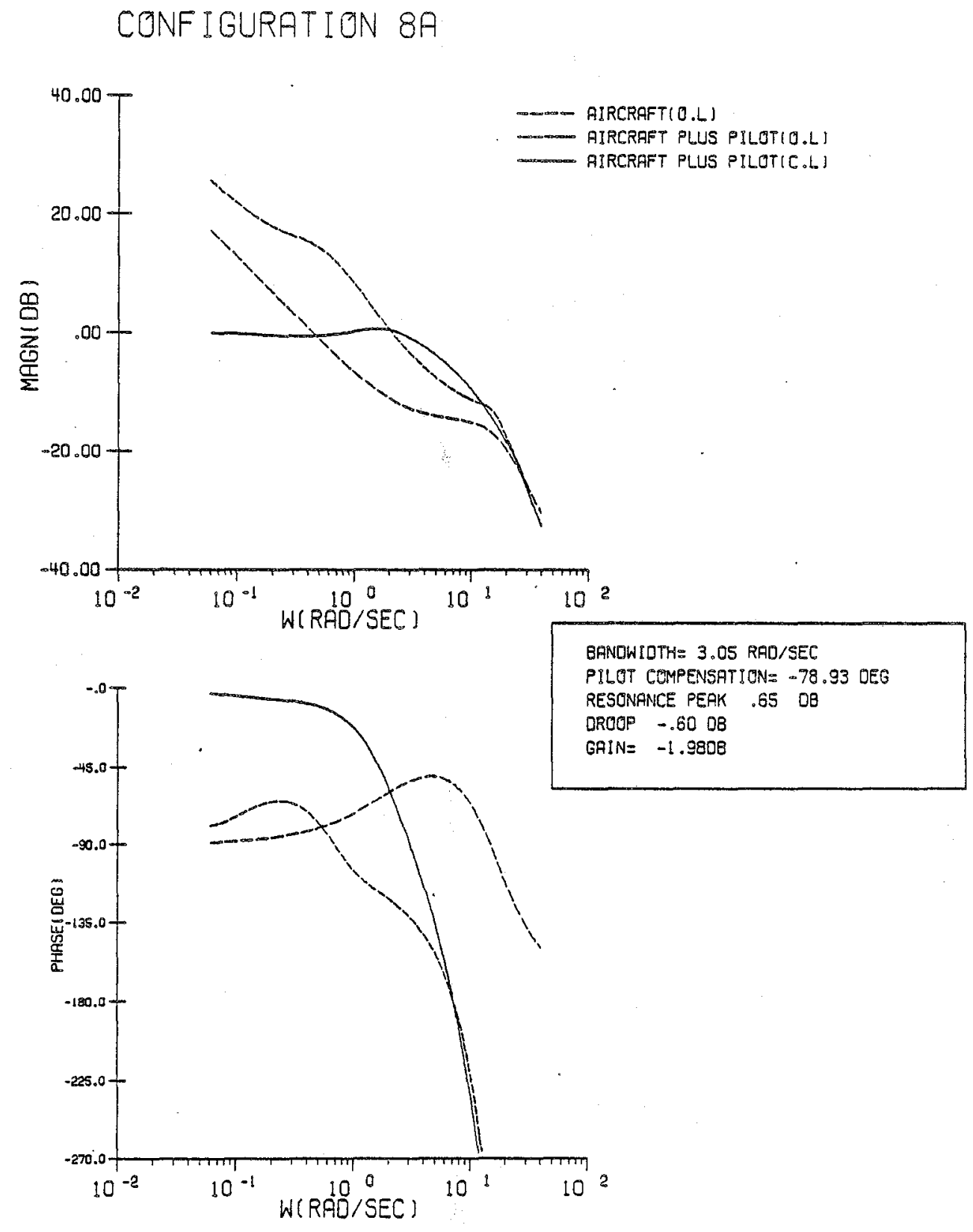

Figure C.66 Configuration $8 \mathrm{~A} /$ Corrected System Frequency Response 


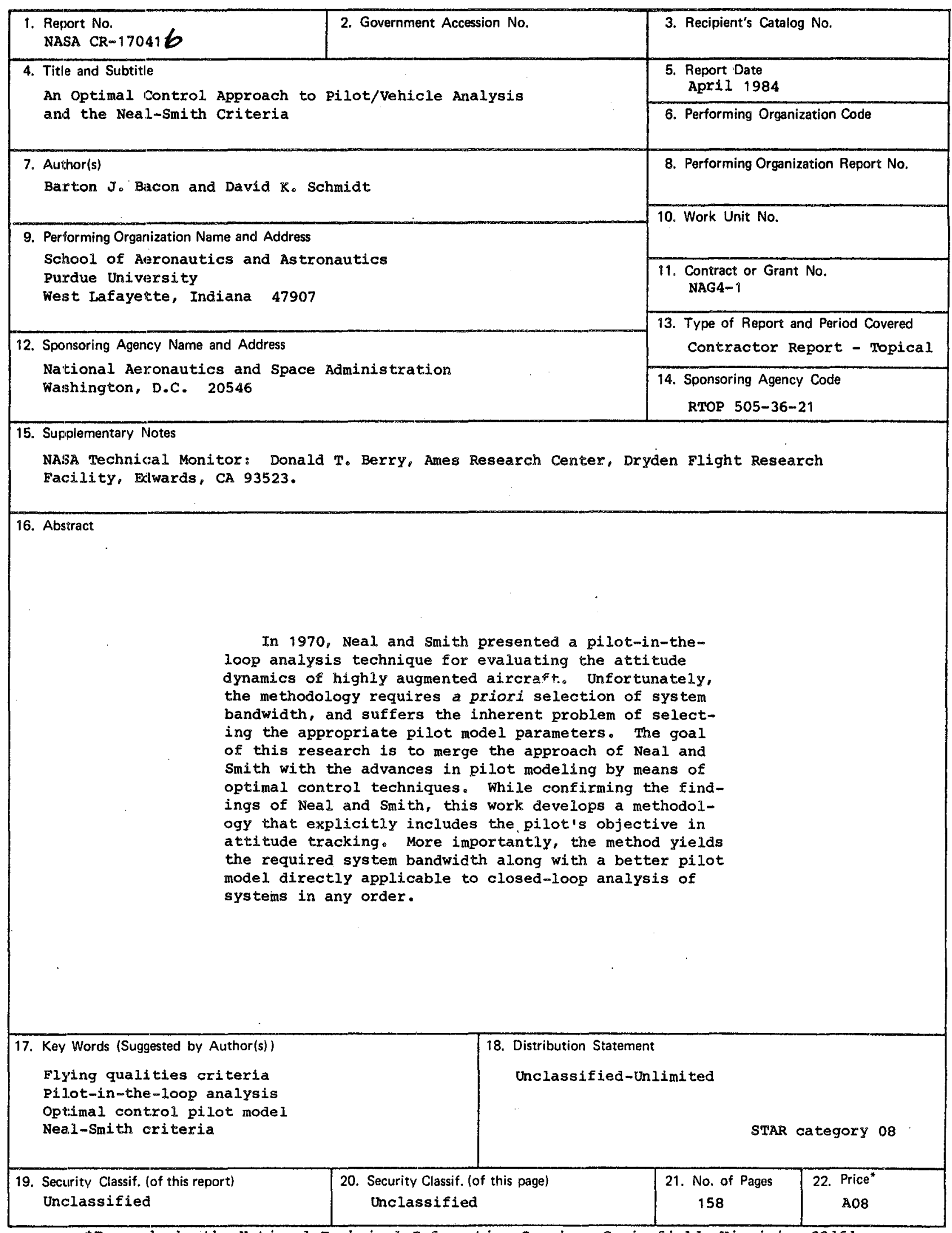

*For sale by the National Technical. Information Service, Springfield, Virginia 22161. 
End of Document 